B Lymphocyte Effector Functions in Health and Disease

\author{
by \\ David John DiLillo \\ Department of Immunology \\ Duke University
}

Date:

Approved:

Thomas F. Tedder, Supervisor

You-Wen He, Chair

Thomas M. Coffman

Michael M. Frank

Garnett Kelsoe

Dissertation submitted in partial fulfillment of

the requirements for the degree of Doctor of Philosophy in the Department of Immunology in the Graduate School

of Duke University 


\section{$\underline{\text { ABSTRACT }}$}

B Lymphocyte Effector Functions in Health and Disease

by

David John DiLillo

Department of Immunology

Duke University

\section{Date:}

Approved:

Thomas F. Tedder, Supervisor

You-Wen He, Chair
Thomas M. Coffman

Thomas M. Coffman

Michael M. Frank

Garnett Kelsoe

An abstract of a dissertation submitted in partial fulfillment of the requirements for the degree of Doctor of Philosophy in the Department of Immunology in the Graduate School of Duke University 
Copyright by

David John DiLillo

2010 


\section{Abstract}

B cells and humoral immunity make up an important component of the immune system and play a vital role in preventing and fighting off infection by various

pathogens. B cells also have been implicated in the pathogenesis of autoimmune disease. However, the various functions that B cells perform during the development and maintenance of autoimmune conditions remain unclear. Therefore, the overall goal of this dissertation was to determine what roles B cells play during autoimmune disease.

In the Chapter 3 of this dissertation, the function of B cells was assessed during tumor immunity, a model of immune system activation and cellular immunity. To quantify B cell contributions to T cell-mediated anti-tumor immune responses, mature B cells were depleted from wild type adult mice using CD20 monoclonal antibody (mAb) prior to syngeneic B16 melanoma tumor transfers. Remarkably, subcutaneous (s.c.) tumor volume and lung metastasis were increased two-fold in B cell-depleted mice. Effector-memory and interferon (IFN) $\gamma$ or tumor necrosis factor (TNF) $\alpha$-secreting CD4 ${ }^{+}$ and $\mathrm{CD}^{+} \mathrm{T}$ cell induction was significantly impaired in B cell-depleted mice with tumors. Tumor antigen (Ag)-specific $\mathrm{CD}^{+} \mathrm{T}$ cell proliferation was also impaired in tumor-bearing mice that lacked B cells. Thus, B cells were required for optimal T cell activation and cellular immunity in this in vivo non-lymphoid tumor model.

In Chapter 4 of this dissertation, the roles that B cells play during immune responses elicited by different allografts were assessed, since allograft rejection is thought to be T cell-mediated. The effects of B cell-depletion on acute cardiac rejection, chronic renal rejection, and skin graft rejection were compared using CD20 or CD19 mAbs. Both CD20 and CD19 mAbs effectively depleted mature B cells, while CD19 mAb treatment depleted plasmablasts and some plasma cells. B cell depletion did not 
affect acute cardiac allograft rejection, although CD19 mAb treatment prevented allograft-specific IgG production. Nonetheless, CD19 mAb treatment significantly reduced renal allograft rejection and abrogated allograft-specific IgG development, while CD20 mAb treatment did not. By contrast, B cell depletion exacerbated skin allograft rejection and augmented the proliferation of adoptively transferred alloAg-specific $\mathrm{CD}^{+} \mathrm{T}$ cells, demonstrating that $\mathrm{B}$ cells can also negatively regulate allograft rejection. Thereby, B cells can either positively or negatively regulate allograft rejection depending on the nature of the allograft and the intensity of the rejection response.

Serum antibody $(\mathrm{Ab})$ is, at least in part, responsible for protection against pathogens and tissue destruction during autoimmunity. In Chapter 5 of this dissertation, the mechanisms responsible for the maintenance of long-lived serum Ab levels were examined, since the relationship between memory B cells, long-lived plasma cells, and long-lived humoral immunity remains controversial. To address the roles of B cell subsets in the longevity of humoral responses, mature B cells were depleted in mice using CD20 mAb. CD20 ${ }^{+}$B cell depletion prevented humoral immune responses and class switching, and depleted existing and adoptively-transferred B cell memory. Nonetheless, B cell depletion did not affect serum Ig levels, Ag-specific Ab titers, or bone marrow (BM) Ab-secreting plasma cell numbers. Co-blockade of LFA-1 and VLA-4 adhesion molecules temporarily depleted long-lived plasma cells from the BM. CD20 $0^{+}$B cell depletion plus LFA-1/VLA-4 mAb treatment significantly prolonged Ag-specific plasma cell depletion from the BM, with a significant decrease in Ag-specific serum IgG. Collectively, these results indicate that BM plasma cells are intrinsically long-lived. Further, these studies now demonstrate that mature and memory B cells are not required for maintaining BM plasma cell numbers, but are required for repopulation of plasma 
cell-deficient BM. Thereby, depleting mature and memory B cells does not have a dramatic negative effect on pre-existing Ab levels.

Collectively, the studies described in this dissertation demonstrate that B cells function through multiple effector mechanisms to influence the course and intensity of normal and autoreactive immune responses: the promotion of cellular immune responses and $\mathrm{CD}^{+} \mathrm{T}$ cell activation, the negative regulation of cellular immune responses, and the production and maintenance of long-lived Ag-specific serum Ab titers. Therefore, each of these three B cell effector mechanisms can contribute independently or in concert with the other mechanisms to clear pathogens or cause tissue damage during autoimmunity. 


\section{Dedication}

This dissertation is dedicated to my wife, Meghan, without whose love and support none of this work could have been accomplished. I also dedicate this

dissertation to my parents, John and MaryAnn, who have relentlessly pushed me to achieve greatness and worked tirelessly to provide me with the resources necessary to succeed. 


\section{Contents}

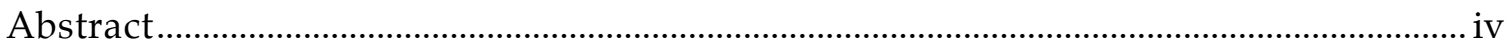

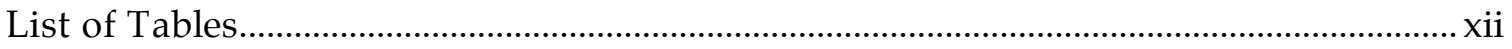

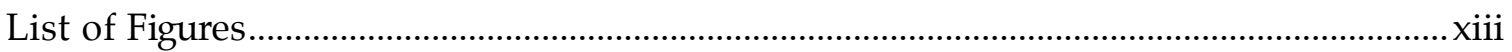

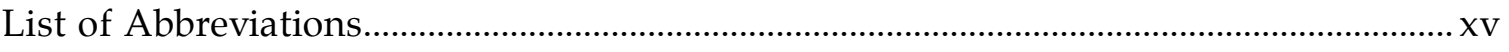

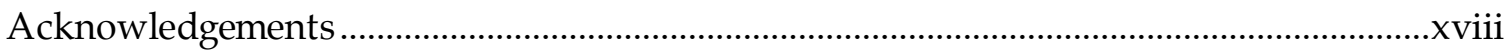

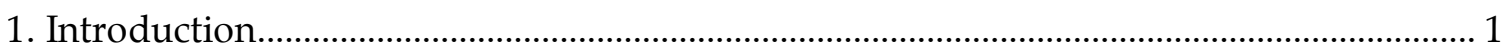

1.1 The Immune System............................................................................................... 1

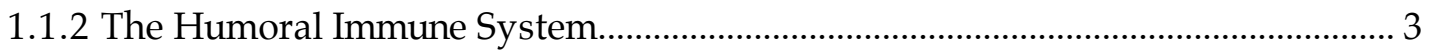

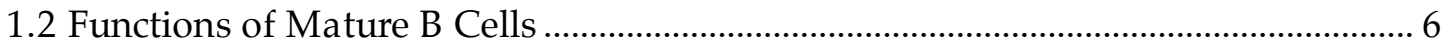

1.2.1 Positive Regulation of Immune Responses by Mature B Cells................................ 6

1.2.1.2 B Cells Regulate Lymphoid Tissue Neogenesis and Structure ...................... 9

1.2.1.3 Regulating DC Function and T Helper Cell Differentiation.......................... 9

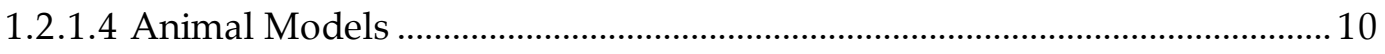

1.2.2 Negative Regulation of Immune Responses by Mature B Cells ........................... 11

1.2.2.1 B10 Cells and Regulatory B Cell Subsets........................................................ 11

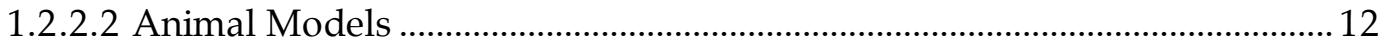

1.2.3 Memory B Cell Contributions to Serum Ab Levels and Terminally-

Differentiated Plasma Cell Numbers .............................................................................. 15

1.2.3.1 Memory B Cell-Dependent Models of Humoral Memory ............................ 17

1.2.3.2 Long-Lived Plasma Cells ............................................................................... 18

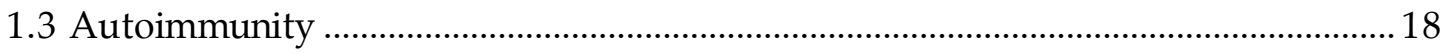

1.3.1 Humoral and Cellular Immunity in Autoimmune Disease .................................... 19

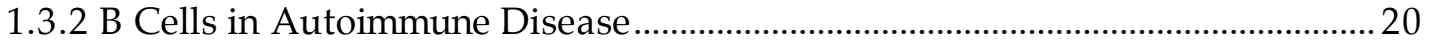

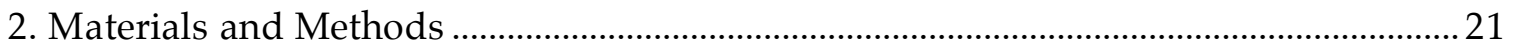

viii 
2.1 Mice

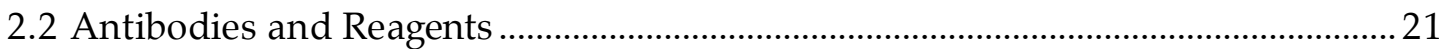

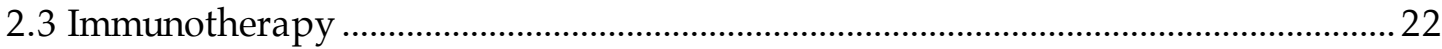

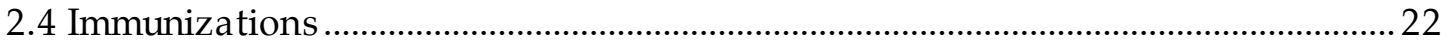

2.5 Cell Preparation and Immunofluorescence Analysis ................................................... 23

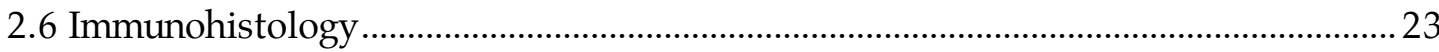

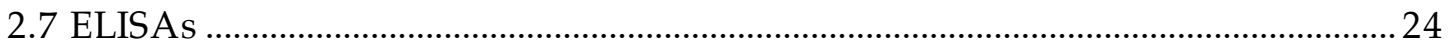

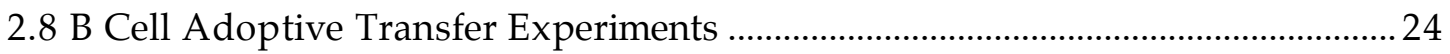

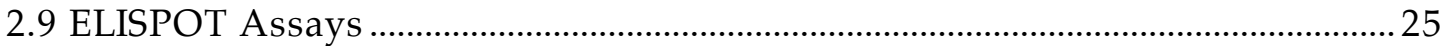

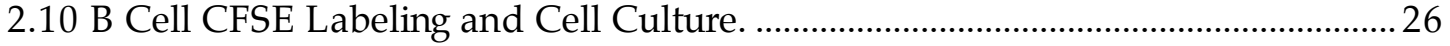

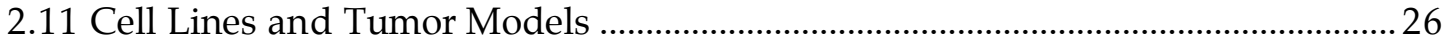

2.12 Measurement of Tumor-Specific Ab Production.......................................................2

2.13 T Cell Adoptive Transfer Experiments ..................................................................... 28

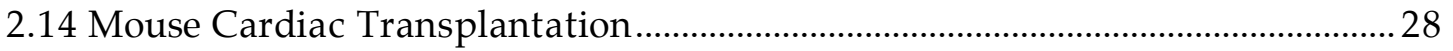

2.15 Measurement of Allograft-Specific Ab Production ................................................29

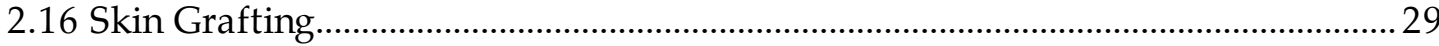

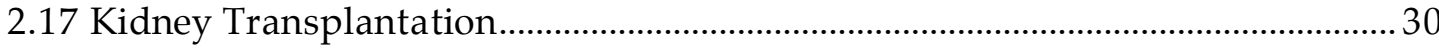

2.18 Kidney Histopathologic and Immunohistology Studies ......................................... 30

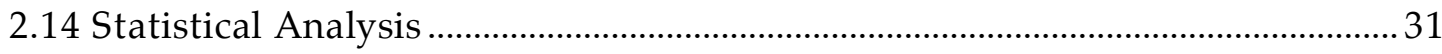

3. B Cells Are Required for Optimal $\mathrm{CD}^{+}$and $\mathrm{CD}^{+} \mathrm{T}$ Cell Tumor Immunity: Therapeutic B Cell Depletion Enhances B16 Melanoma Growth in Mice.............................. 32

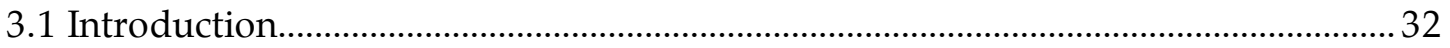

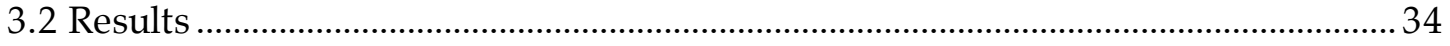

3.2.1 B Cell Depletion Rnhances B16 Melanoma Growth and Metastasis ................. 34

3.2.2 CD20 mAb Rffectively Depletes B Cells in Tumor-Bearing Mice ........................39 
3.2.3 Anti-tumor $\mathrm{Ab}$ responses in $\mathrm{B} 16$ / F0 / OVA-bearing mice.

3.2.4 T Cell Numbers are Reduced Following B Cell Depletion. 42

3.2.5 B Cell Depletion Impairs $\mathrm{CD}^{+}$and $\mathrm{CD} 8^{+}$Effector-Memory T Cell Induction 46

3.2.6 B Cell Depletion Impairs IFN $\gamma$ and TNF $\alpha$ Induction by $\mathrm{CD}^{+}$and $\mathrm{CD} 8^{+} \mathrm{T}$

Cells In Vivo 50

3.2.7 B Cells Are Required for Optimal T Cell Cytokine Induction In Vitro..... 54

3.2.8 B cell Depletion Impairs Tumor Ag-Specific CD8 ${ }^{+} \mathrm{T}$ Cell Proliferation In Vivo 56

3.2.9 B Cell Depletion Does Not Affect the Growth of Established Tumors .57

3.3 Discussion 59

4. B Lymphocytes Differentially Influence Acute and Chronic Allograft Rejection in Mice . .64

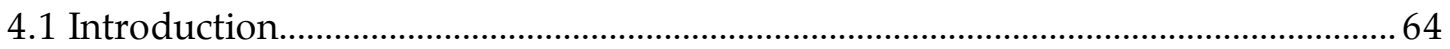

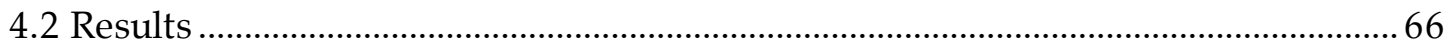

4.2.1 CD20 and CD19 mAbs Effectively Deplete Different B Cell Populations ...... 66

4.2.2 CD19 mAb, But Not CD20 mAb Treatment Depletes Serum IgG in Naïve Mice. 70

4.2.3 CD19 mAb Treatment Depletes Ab-Secreting Plasma Cells ............................... 72

4.2.4 CD19 mAb Treatment Inhibits Graft-Specific IgG Generation during Cardiac

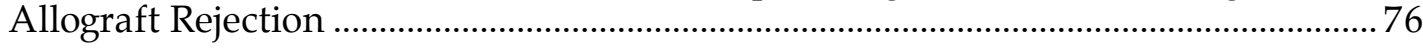

4.2.5 CD20 mAb Treatment Impairs Skin Allograft-Specific IgG Generation ............78

4.2.6 CD20 mAb Treatment Accelerates Minor Ag-Mismatched Skin Allograft

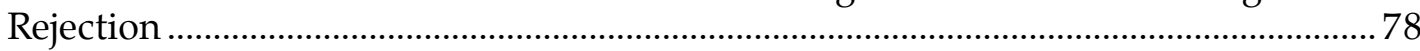

4.2.7 CD19 mAb Treatment Prevents Chronic Renal Allograft Rejection................... 81

4.2.8 CD19 mAb Treatment Depletes Pre-Existing Allograft-Specific IgG................ 89

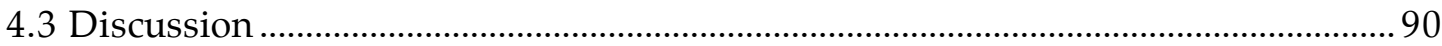

5. Maintenance of Long-Lived Plasma Cells and Serological Memory Despite Mature and Memory B Cell Depletion During CD20 Immunotherapy In Mice.. 


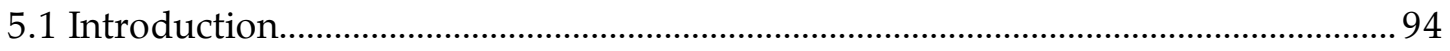

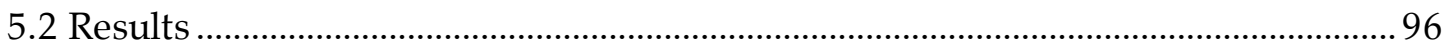

5.2.1 Serum Ig Levels Following CD20 ${ }^{+}$B Cell Depletion ............................................. 96

5.2.2 Ab Responses Following CD20 ${ }^{+}$B Cell Depletion ................................................99

5.2.3 GC B Cells are Depleted by CD20 mAb Treatment ............................................103

5.2.4 Memory B Cells and Ab Responses Are Depleted by CD20 mAb ...................106

5.2.5 CD20 Expression by Plasmablasts and Plasma Cells In Vitro and In Vivo .110

5.2.6 ASCs Resist CD20 mAb-Mediated Depletion In Vivo.....................................111

5.2.7 Long-Lived Plasma Cell Depletion .........................................................................113

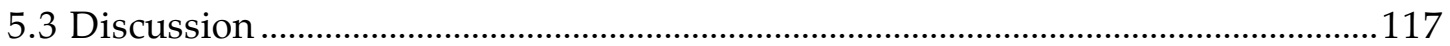

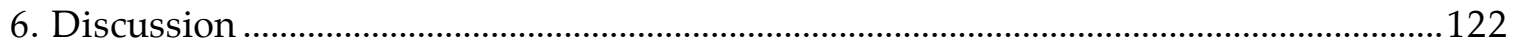

6.1 B Cell Functions during Autoimmune Disease .......................................................... 122

6.1.1 B Cell Promotion of Autoimmune Disease ............................................................122

6.1.2 B Cell Negative Regulation of Autoimmune Disease ..........................................123

6.1.3 Ab Functions during Autoimmunity ……......................................................... 124

6.2 B cell effector mechanisms are not mutually exclusive.............................................125

6.3 Remaining Questions Regarding B Cell Effector Functions .....................................128

6.3.1 Potential Factors Influencing How B Cells Function during Autoimmunity ..128

6.3.2 Do B Cell Subsets Carry Out These Effector Functions? .....................................130

6.4 B Cell Effector Functions in Human Autoimmune Disease.....................................131

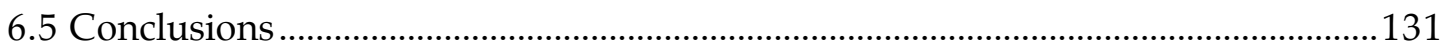

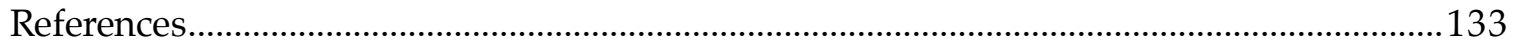

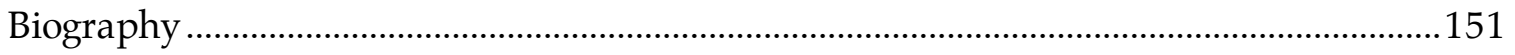




\section{List of Tables}

Table 1. $\mathrm{CD}^{+}$and $\mathrm{CD}^{+}$total and naïve $\mathrm{T}$ cell numbers in tumor-bearing mice............... 44

Table 2. B cell subsets in CD20 and CD19 mAb-treated mice...........................................69

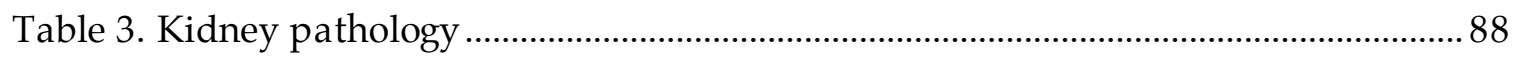

Table 4. Immunization effects on CD20 mAb-induced B cell depletion ............................105 


\section{List of Figures}

Figure 1. The Humoral, Cellular, and Innate Immune Systems. .............................................. 2

Figure 2. B cell activation, GC and memory B cell development, and plasma cell differentiation. 5

Figure 3. B cells influence multiple immune functions........................................................... 7

Figure 4. Mechanisms of Serological Memory Maintenance................................................... 16

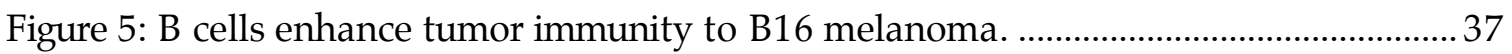

Figure 6: B cell depletion is effective in mice given B16 melanoma.................................... 41

Figure 7: B cell depletion reduces spleen $\mathrm{CD}^{+} \mathrm{T}$ cell numbers............................................. 43

Figure 8: Effector-memory T cell induction is impaired in B cell-depleted tumor-bearing mice.

Figure 9: B cell depletion impairs the induction of IFN $\gamma$ - and TNF $\alpha$-secreting $\mathrm{CD}^{+}$cells in tumor-bearing mice.

Figure 10: B cell depletion impairs the induction of IFN $\gamma$ - and TNF $\alpha$-secreting $\mathrm{CD}^{+}$cells in tumor-bearing mice.

Figure 11: B cells are required for optimal $\mathrm{T}$ cell cytokine induction in vitro.

Figure 12: B cell depletion impairs tumor Ag-specific $\mathrm{CD}^{+} \mathrm{T}$ cell proliferation, but does not affect the growth of pre-established tumors

Figure 13. CD20 and CD19 mAbs effectively deplete mature B cells in vivo. 68

Figure 14. CD19 mAb treatment depletes serum IgG, while CD20 mAb treatment does not.

Figure 15. CD19 mAb treatment depletes Ab-secreting plasma cells.................................74

Figure 16. CD19 mAb treatment impairs allograft-specific IgG production........................77

Figure 17. CD20 mAb treatment impairs allograft-specific IgG generation, but accelerates minor histocompatibility-mismatched skin allograft rejection and alloAg-specific CD $4^{+}$

T cell proliferation.

Figure 18. CD19 mAb treatment prevents renal allograft rejection, impairs allograftspecific IgG generation, and reduces pre-existing serum IgG levels. 
Figure 19. CD19 mAb treatment reduces renal injury and C4d deposition following renal

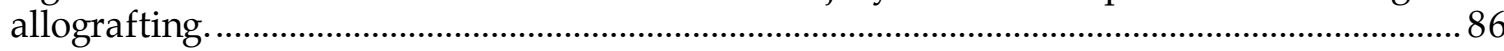

Figure 20: Humoral immunity following CD20+ $\mathrm{B}$ cell depletion..........................................98

Figure 21: TD and TI Ab responses in CD20 ${ }^{+} \mathrm{B}$ cell depleted mice. .................................101

Figure 22: GCs in B cell depleted mice. ............................................................................... 104

Figure 23: Long-lived Ag-specific Ab levels and memory Ab responses in CD20+ $\mathrm{B}$ cell-

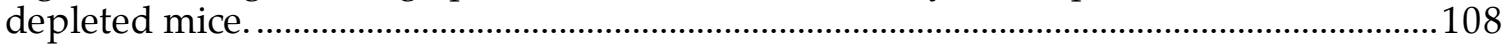

Figure 24: CD20 expression by plasmablasts and ASCs...................................................112

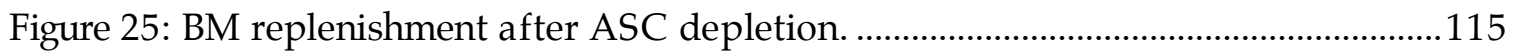

Figure 26. B cell effector mechanisms during immune responses.......................................127 


\section{List of Abbreviations}

\begin{tabular}{|c|c|}
\hline $\mathrm{Ab}$ & Antibody \\
\hline $\mathrm{Ag}$ & Antigen \\
\hline $\mathrm{APC}$ & Antigen presenting cell \\
\hline ASC & Antibody-secreting cell \\
\hline autoAbs & Autoantibodies \\
\hline BCR & B cell antigen receptor \\
\hline $\mathrm{BD}$ & Becton Dickinson \\
\hline BrdU & 5-bromo-2-deoxyuridine \\
\hline $\mathrm{BM}$ & Bone marrow \\
\hline BSA & Bovine serum Albumin \\
\hline CFSE & Carboxyfluorescein Succinimidyl Ester \\
\hline CGG & Chicken gamma globulin \\
\hline CHS & Contact hypersensitivity \\
\hline CIA & Collagen-induced arthritis \\
\hline $\mathrm{d}$ & day \\
\hline $\mathrm{DC}$ & Dendritic cell \\
\hline DNA & Deoxyribonucleic acid \\
\hline DNP & Dinitrophenyl \\
\hline DTH & Delayed-type hypersensitivity \\
\hline EAE & Experimental autoimmune encephalomyelitis \\
\hline ELISA & Enzyme-linked immunosorbent assay \\
\hline ELISPOT & Enzyme-linked immunospot \\
\hline FITC & Fluorescein isothiocyanate \\
\hline
\end{tabular}




\begin{tabular}{|c|c|}
\hline GC & Germinal center \\
\hline GFP & Green fluorescence protein \\
\hline $\mathrm{h}$ & hour \\
\hline $\mathrm{H} \& \mathrm{E}$ & Hematoxylin and Eosin \\
\hline HRP & Horseradish peroxidase \\
\hline huCD19Tg & Human CD19 Transgenic \\
\hline IFN & Interferon \\
\hline i.p. & intraperitoneal \\
\hline $\operatorname{Ig}$ & Immunoglobulin \\
\hline i.v. & intravenous \\
\hline IVIG & Intravenous Immunoglobulin \\
\hline IL & Interleukin \\
\hline LN & Lymph Node \\
\hline LPS & Lipopolysaccharide \\
\hline $\mathrm{mAb}$ & monoclonal Antibody \\
\hline MFI & Mean fluorescence intensity \\
\hline MHC & Major histocompatibility complex \\
\hline mo & month \\
\hline mOVA & membrane-bound OVA \\
\hline MZ & Marginal Zone \\
\hline NOD & Non-obese diabetic \\
\hline NP & 4-hydroxy-3-nitrophenyl acetyl \\
\hline OCT & Optimal cutting temperature \\
\hline OD & Optical density \\
\hline OVA & Ovalbumin \\
\hline
\end{tabular}




$\begin{array}{ll}\text { PAS } & \text { Periodic Acid Schiff } \\ \text { PBS } & \text { Phosphate-buffered saline } \\ \text { PE } & \text { Phycoerythrin } \\ \text { RPMI } & \text { Roswell Park Memorial Institute } \\ \text { s.c. } & \text { subcutaneous } \\ \text { SEM } & \text { Standard error of the mean } \\ \text { SS } & \text { single-stranded } \\ \text { TCR } & \text { T cell antigen receptor } \\ \text { TD } & \text { T cell-dependent } \\ \text { TI } & \text { T cell-independent } \\ \text { TLR } & \text { Toll-like receptor } \\ \text { TNF } & \text { Tumor necrosis factor } \\ \text { TNP } & \text { Trinitrophenyl }\end{array}$




\section{Acknowledgements}

With extreme gratitude, I would first like to thank my mentor, Dr. Thomas Tedder, for everything he has taught me during the past 5 years. I am truly lucky to have worked for such an outstanding, intelligent, and kind investigator. I am especially thankful to Tom for all of the time he has given and patience he has shown while teaching me how to become a great and productive scientist. I appreciate all of the motivation he has supplied me with, especially during the trials and tribulations that come with graduate school. It has truly been a pleasure to work for someone who is so excited and energized about scientific discovery.

I would also like to thank my committee members, Dr. Garnett Kelsoe, Dr. Thomas Coffman, Dr. Michael Frank, and Dr. You-Wen He, for their guidance, support, and collaboration over the past 5 years.

All of the past and present members of the Tedder Laboratory have been instrumental in my success as a graduate student, and I must especially thank Dr. Jon Poe, Dr. Karen Haas, and Dr. Bill Venturi for all their invaluable guidance, critiques, and contributions to my work in the laboratory.

I would also like to thank the members of the Duke University Immunology Class of 2004, Derek Cain, Matt Holl, Ana Sanchez, Alexis Dunkle, and Claire Gordy, for their friendship, support, and good times shared together over the past 6 years.

Finally, I would like to thank my incredible family for all that they have done for me. First, I must thank my wife, Meghan, who was always the first to hear about the successful experiments, but who was also there for me on the more difficult days of graduate school. I could not possibly have completed this tremendous task without her unconditional and never-ending love and support. I also would not be where I am today 
without the love and guidance of my family. My parents pushed me every day of my life to give my best effort in everything that I do. They worked incredibly hard and sacrificed so that I would have everything that I needed to achieve success in life, and I will be forever grateful to them. 


\section{Introduction}

\subsection{The Immune System}

The immune system is designed to protect the host from predation by invading foreign microorganisms and is composed of two main arms: the innate and the adaptive immune systems. The innate immune response is the first line of defense against invading pathogens and is made up of numerous cell types, including dendritic cells (DCs) and macrophages. These cells eliminate pathogens by phagocytosing them, among other effector mechanisms. The phagocytosed foreign pathogen is degraded and its protein components are processed and presented to cells of the adaptive immune system to elicit an adaptive immune response (1). The adaptive immune system is made up of two components: humoral immunity, which is carried out by B lymphocytes, and cellular immunity, which is carried out by T lymphocytes (Figure 1). Unlike the innate immune system, each individual B and T lymphocyte bears a unique surface receptor recognizing a different antigen $(\mathrm{Ag})$, which is a molecular determinant expressed by foreign or self proteins. Therefore, an incredible diversity of Ag, and thus foreign pathogens, may be recognized by these cells. An adaptive immune response also forms a pool of long-lived memory B and T lymphocytes that can rapidly respond upon secondary exposure to a pathogen (2). 


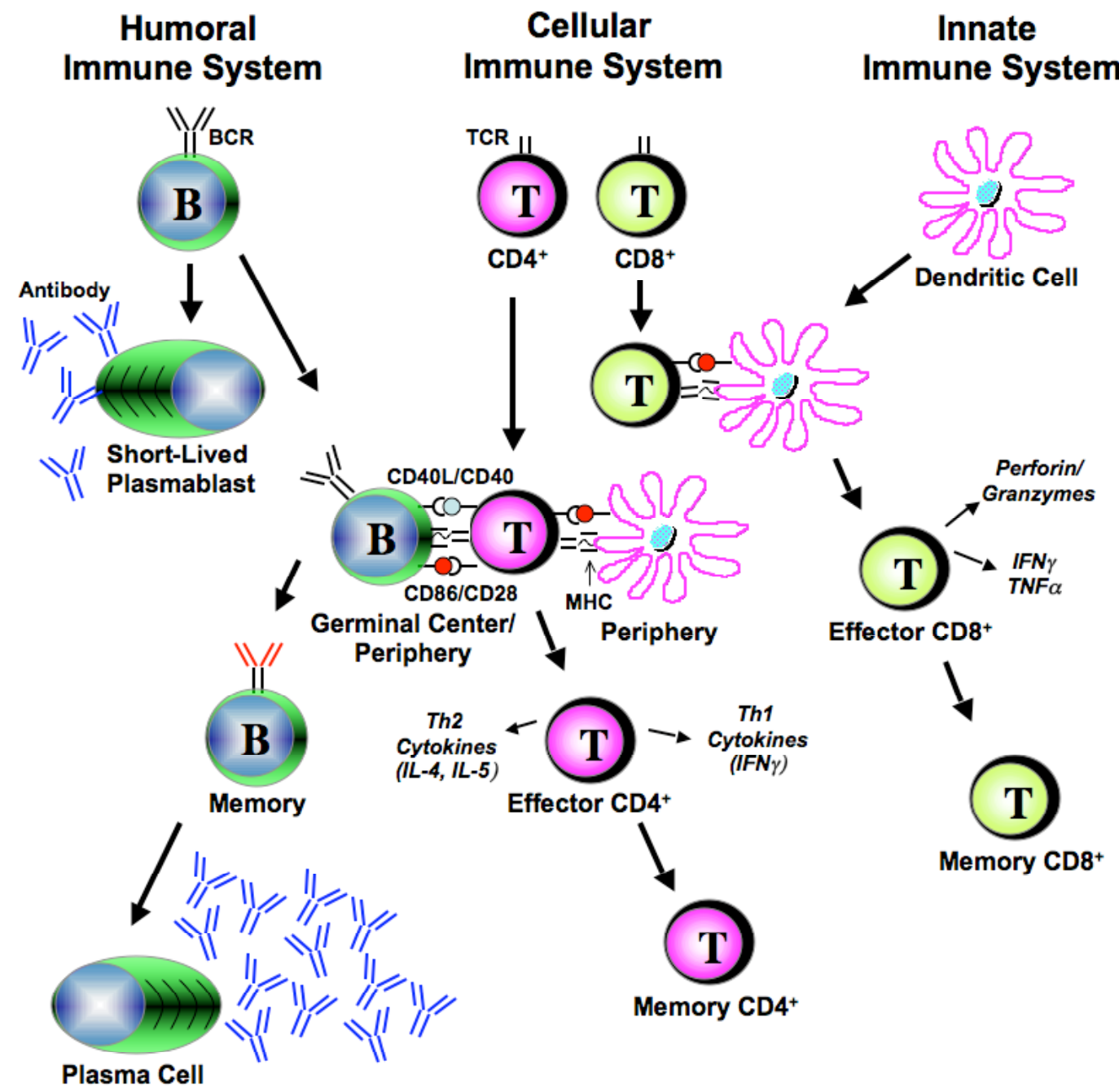

Figure 1. The Humoral, Cellular, and Innate Immune Systems. The innate immune system is made up of cells, such as dendritic cells, that capture and present Ag to CD4 ${ }^{+}$ and $\mathrm{CD}^{+} \mathrm{T}$ cells, the components of cellular immunity. Activated T cells carry out their effector functions and then form a long-lived pool of memory cells. The humoral immune system is comprised of B cells, which when activated, can differentiate into plasmablasts that secrete Ag-specific Ab. B cells can interact with helper CD4 ${ }^{+} \mathrm{T}$ cells to promote $\mathrm{B}$ cell activation, the germinal center reaction, memory B cell formation, and plasma cell differentiation. 


\subsubsection{The Cellular Immune System}

The responsibilities of the cellular immune system are carried out by $\mathrm{T}$ lymphocytes, which can be subdivided into two populations: $\mathrm{CD} 4^{+}$helper $\mathrm{T}$ cells and $\mathrm{CD}^{+}$cytotoxic $\mathrm{T}$ cells. $\mathrm{T}$ cells become activated when their T cell Ag receptor (TCR) binds to a peptide:major histocompatibility complex (MHC) presented on the surface of a target cell. $\mathrm{CD}^{+} \mathrm{T}$ cells recognize peptide:MHC-II complexes expressed on DCs, macrophages, and B cells that have phagocytosed, processed, and presented a foreign Ag. Upon activation, $\mathrm{CD} 4^{+} \mathrm{T}$ cells release an array of cytokines that influence the immune response. Among many other effector molecules, Th1-type CD4 ${ }^{+} \mathrm{T}$ cells produce the pro-inflammatory cytokine tumor necrosis factor (TNF) $\alpha$ and direct macrophages to become activated by producing interferon (IFN) $\gamma$. Th2-type $\mathrm{CD}^{+} \mathrm{T}$ cells promote antibody $(\mathrm{Ab})$ production by B cells by secreting cytokines such as interleukin (IL)- 4 . $\mathrm{CD}^{+}$helper T cells also function during the germinal center (GC) B cell response by providing other signals necessary for B cell survival and differentiation (3). $\mathrm{CD}^{+} \mathrm{T}$ cells recognize peptide:MHC-I complexes expressed on the surface of any cell in the body that has processed and presented proteins derived from that same cell. Thus, these cells function to kill cells that have been invaded by a foreign pathogen before the infection spreads to other healthy cells. Upon activation, the $\mathrm{CD}^{+}$cell releases lytic granules specifically into the target cell that expresses the foreign peptide on its surface, thereby specifically killing that cell while sparing healthy cells (4). $\mathrm{CD}^{+} \mathrm{T}$ cells also produce inflammatory cytokines, such as IFN $\gamma$.

\subsubsection{The Humoral Immune System}

B lymphocytes make up the humoral immune system, with terminallydifferentiated plasma cells representing the effector cells of humoral immunity. B cell development begins in the bone marrow $(\mathrm{BM})$, where stem cells receive the appropriate 
signals and differentiate into progenitor and precursor B cells, the stages of B cell development when the B cell Ag receptor (BCR) heavy and light chains rearrange, respectively, to form a functional BCR (Figure 2, ref. 5). Upon differentiation to the immature B cell stage, the BCR is expressed on surface of the cell. When they leave the $\mathrm{BM}$, immature B cells migrate to the periphery and recirculate or settle in various lymphoid tissues, including the spleen and lymph nodes (LNs) as naïve mature B cells (5).

Upon binding its cognate $\mathrm{Ag}$, a naïve $\mathrm{B}$ cell internalizes and processes the $\mathrm{Ag}$ and presents it to a cognate helper $\mathrm{T}$ cell in the periphery of the lymphoid follicle. After receiving the appropriate signals from the helper $\mathrm{T}$ cell, the B cell may rapidly differentiate into short-lived plasma cells that secrete Ag-specific germline-encoded antibodies mainly of the IgM isotype $(6,7)$. In addition, the B cell proliferates and converts the follicle into a GC. Here, the proliferating B cells undergo affinity maturation - the process by which GC B cells undergo somatic hypermutation and clonal selection (8). Mutations in the binding regions of the B cells' Ag receptors result in B cell clones of either higher or lower affinities for the Ag, and those B cell clones with the highest affinities are selected to survive and become memory B cells and plasma cells (9-11). Memory B cells persist for extended periods of time after Ag challenge, and rapidly expand and differentiate into plasma cells during secondary responses (12). Thus, postGC memory B cells give rise to Ab isotype-switched plasmablasts and plasma cells once outside of GCs. 


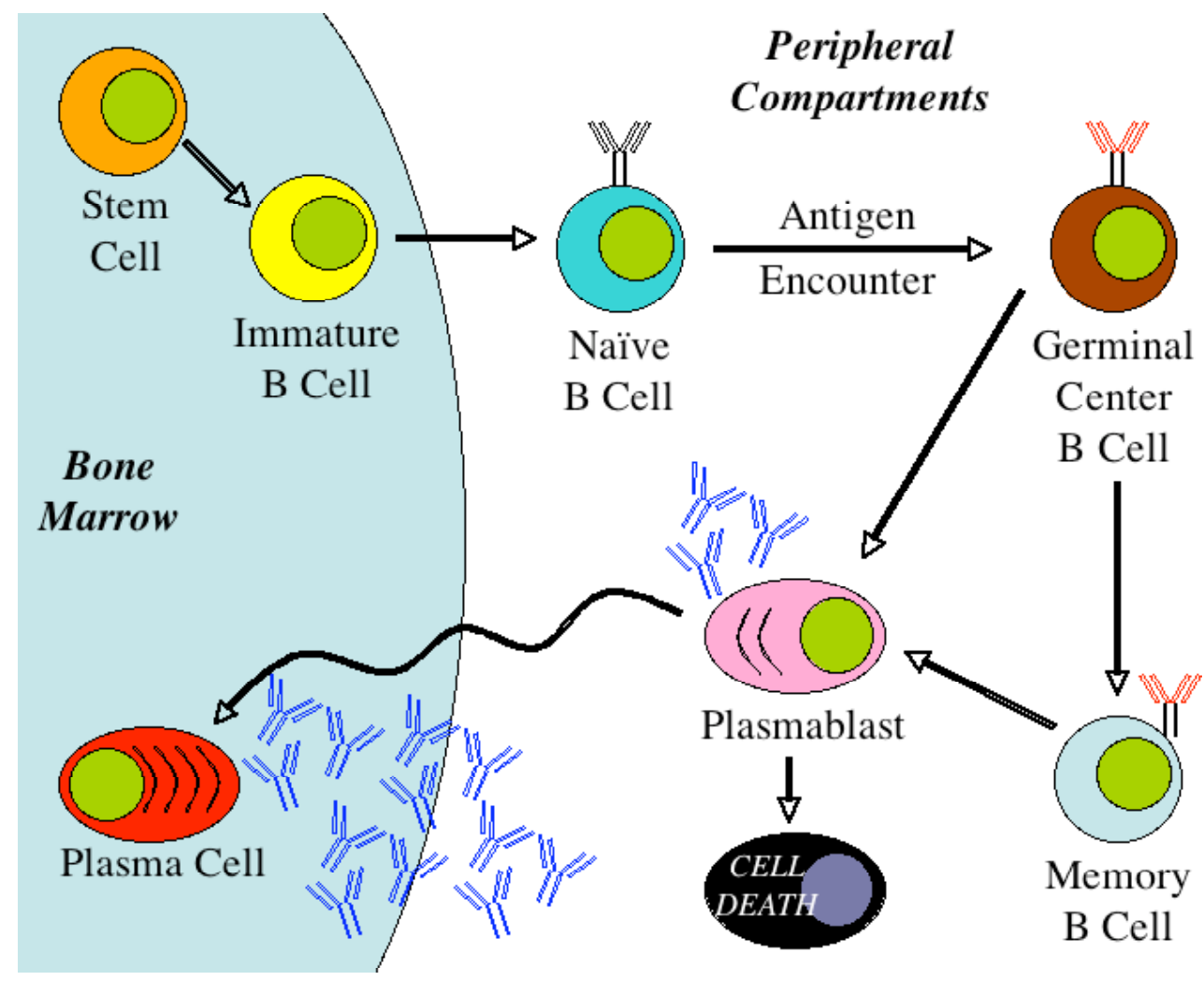

Figure 2. B cell activation, GC and memory B cell development, and plasma cell differentiation. 


\subsection{Functions of Mature B Cells}

\subsubsection{Positive Regulation of Immune Responses by Mature B Cells}

B cells can positively influence immune responses through numerous mechanisms other than Ab production (Figure 3). B cells can function as potent Ag-presenting cells and provide critical costimulatory signals to T cells. B cells also regulate lymphoid tissue neogenesis and structure. In addition, DCs, macrophages, and T helper cell differentiation decisions may all be impacted by B cells during the development of immune responses. 


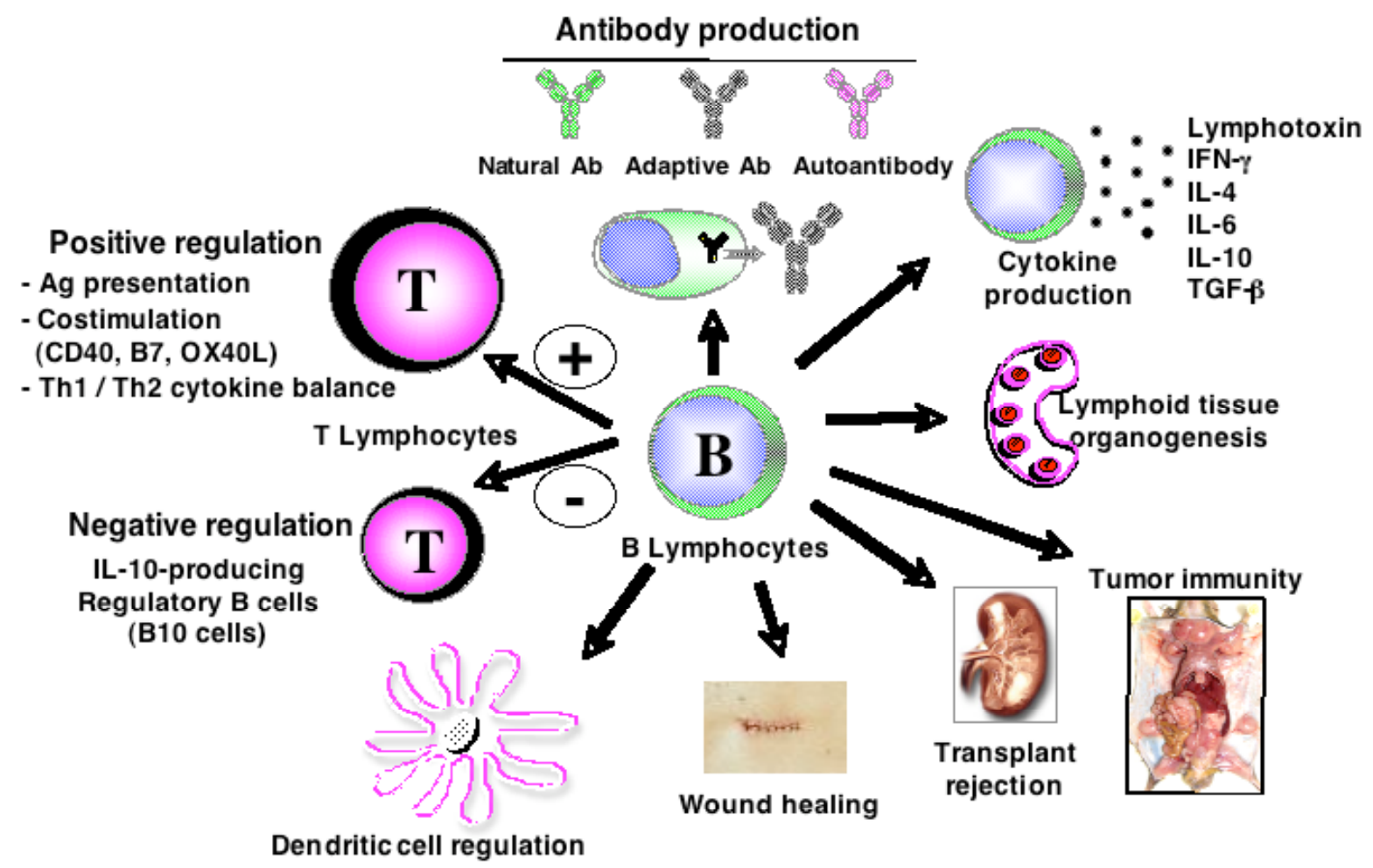

Figure 3. B cells influence multiple immune functions. 


\subsubsection{Ag Presentation and Costimulation}

B cells are recognized as important Ag-presenting cells during the initiation of immune responses $(13,14)$ based on experiments in mice that were given anti-IgM Ab to deplete B cells from birth $(13,15-17)$ or experiments in mice with a genetic defect in B cell development (18). The absence of B cells impaired LN T cell priming in response to $\mathrm{KLH} / \mathrm{CFA}$ immunization, but priming can be rescued though the adoptive transfer of purified B cells (16). B cell deficient mice also show impaired $\mathrm{CD} 4^{+}$memory $\mathrm{T}$ cell formation (19). Ag presentation occurs in an Ag-specific manner, since the adoptive transfer of hapten-specific B cells enhances $\mathrm{T}$ cell proliferative responses to that hapten coupled to carrier (13). Direct evidence for B cells functioning as Ag-presenting cells comes from experiments in which mice lacking MHC-II expression in only B cells showed impaired $\mathrm{CD}^{+} \mathrm{T}$ cell clonal expansion, effector differentiation, and memory formation $(19,20)$. However, while the absence of B cells impairs CD4 ${ }^{+} \mathrm{T}$ cell priming in many studies $(16,17,19-21)$, other studies have indicated that B cells play no role in CD4 ${ }^{+} \mathrm{T}$ cell activation (22-26). These discrepancies may be due to the imperfect experimental systems used in which mice are rendered B cell deficient from birth, which profoundly impacts the development of the immune system. Recent studies assessing B cell function during cellular immune responses have clarified this situation by utilizing CD20 monoclonal Abs (mAbs) to specifically deplete mature CD20+ $\mathrm{B}$ cells from mice with otherwise intact immune systems. These studies found that B cells are necessary for optimal $\mathrm{CD}^{+} \mathrm{T}$ cell activation during immune responses to low dose Ags, with DCs (DCs) sharing this duty (27). When B cell-depleted mice are challenged with a high-dose of $\mathrm{Ag}$, a role for B cells is not seen. However, autoAg challenge in B cell-deficient mice also results in reduced Ag-specific $\mathrm{CD}^{+} \mathrm{T}$ cell activation, clearly underlining a significant contributory role for B cells in autoimmune disease initiation. 
B cells may also stimulate Ag-specific $\mathrm{CD}^{+}{ }^{+} \mathrm{T}$ cell proliferation after $\mathrm{T}$ cells have been primed by DCs (26). B cells and T cells also physically interact (28) via costimulatory molecules such as B7 / CD28, CD40 / CD40 ligand, and OX40 ligand/ OX40 on the surface of B cells and T cells, respectively, and these co-stimulatory signals can determine the extent of the primary expansion of $\mathrm{CD}^{+} \mathrm{T}$ cells in mice $(29,30)$ and in humans (31).

\subsubsection{B Cells Regulate Lymphoid Tissue Neogenesis and Structure}

B cells also regulate lymphoid tissue organization and neogenesis $(32,33)$. The absence of B cells during mouse development results in significant abnormalities within the immune system, including decreased thymocyte numbers and diversity (34) as well as spleen DC and T cell defects $(23,35,36)$. Mice without B cells also lack follicular DCs and several macrophage populations (36-38). Moreover, after B cell depletion in mice, it is not possible to identify histological structures resembling follicles within the spleens, which may prevent normal cell-to-cell interactions in these structures (39).

\subsubsection{Regulating DC Function and T Helper Cell Differentiation}

DCs from genetically B cell-deficient mice produce more IL-12 than DCs from wild type mice, which may prevent T cell IL-4 production and influence T helper cell differentiation (23). B cells can also directly regulate $\mathrm{T}$ helper cell differentiation in vitro, since Ag-specific B cells can induce CD4 ${ }^{+} \mathrm{T}$ cells to produce IL-4, while DCs induce IFN $\gamma$ in the same assays (40). In vivo, B cells are required to induce CD4 ${ }^{+}$effector $\mathrm{T}$ cell responses in a Th2 parasite model (41). Furthermore, the existence of two populations of cytokine-producing effector B cells in mice (called Be1 and Be2) that can influence $\mathrm{CD}^{+} \mathrm{T}$ cell differentiation into Th1 and Th2 subsets, respectively, has also been described (42). 


\subsubsection{Animal Models}

Multiple animal models have been used to assess the roles of B cells during autoimmune disease and immune responses. For example, B cells can positively contribute to disease in murine arthritis models. When B cells are depleted from collagen-induced arthritis (CIA)-susceptible DBA-1 mice before collagen immunization, disease onset and auto $\mathrm{Ab}$ production are delayed and the severity of arthritis is reduced both clinically and histologically (43). While collagen-specific Ab production contributes to disease in this model, B cells are also required for optimal $\mathrm{CD}^{+} \mathrm{T}$ cell activation following collagen immunization (27).

B cells also play a role in the development and disease progression of diabetes (44). B cells are among the earliest cells to infiltrate the pancreatic islets of non-obese diabetic (NOD) mice $(45,46)$, where they can organize along with T cells into lymphoid structures with GCs that may promote the selection of autoreactive B cells (47). Several studies indicate that B cells can serve as Ag-presenting cells or prime $\beta$ cell-specific T cells $(27,48-50)$, and B cell depletion in 5 week-old NOD female mice decreases insulitis and prevents diabetes in $>60 \%$ of littermates (51). In addition, CD20 mAb treatment of 15 week-old NOD female mice significantly delays, but does not prevent or reverse diabetes onset. Protection from diabetes results from impaired $\mathrm{CD} 4^{+} \mathrm{T}$ cell activation in the pancreatic LNs of B cell-depleted NOD mice (27).

Multiple studies have suggested that B cells are important promoters of experimental autoimmune encephalomyelitis (EAE) pathogenesis (52-55), and B cells can also serve as Ag-presenting cells during EAE initiation (56, 57). Further, CD20+ B cell depletion after EAE development dramatically suppresses disease symptoms (58). Thus, activation of $\mathrm{CD}^{+} \mathrm{T}$ cells is a major function of $\mathrm{B}$ cells during EAE. 
B cells also activate T cells during murine lupus. For example, B cell-deficient MRL/lpr mice have no pathology at an age when B cell-intact MRL/lpr mice have fullblown disease (59). There is also a marked reduction in the number of activated/memory T cells in B cell-deficient MRL/lpr mice compared to mice with B cells (60). Therefore, B cells mediate pathology during lupus by activating pathogenic T cells.

\subsubsection{Negative Regulation of Immune Responses by Mature B Cells}

Evidence for B cell negative regulatory function has accumulated over the past 30 years. B cell suppression of an immune response was first reported in 1974, where spleen B cells were found to impair delayed-type hypersensitivity (DTH) responses in guinea pigs $(61,62)$. Specifically, column depletion of B cells from splenocyte preparations eliminated the ability of adoptively transferred cells to inhibit delayedonset skin reactions (61). This finding led to the conclusion that DTH responses and T cell function can be regulated by suppressor B cells that are distinct from Ab-producing cells. Although identifying regulatory B cell subsets and their mechanisms of action has taken over 30 years, regulatory B cells are now recognized as an important new component of the immune system.

\subsubsection{B10 Cells and Regulatory B Cell Subsets}

Over the past decade, B cell negative regulation has been demonstrated in multiple mouse autoimmunity models (63-67). Mizoguchi et al. were the first to use the term "regulatory B cells" to designate B cells with negative regulatory properties (63). B cell regulatory activities and negative regulation through IL-10 production have now been attributed to multiple phenotypically diverse B cell subsets in different mouse models. A relatively rare negative regulatory $\mathrm{B}$ cell subset that is predominantly contained within a phenotypically unique $C D 1 \mathrm{~d}^{\text {hi }} \mathrm{CD} 5^{+} \mathrm{CD} 19^{\text {hi }}$ subset in the spleens of naïve wild type mice 
has been identified (68). This regulatory B cell subset is Ag-specific and significantly influences T cell activation and inflammatory responses through IL-10 production (58, 68). Given that multiple regulatory B cell subsets are likely to exist, as now recognized for $\mathrm{T}$ cells, this IL-10 competent, $\mathrm{CD} 1 \mathrm{~d}^{\text {hi }} \mathrm{CD} 5^{+} \mathrm{CD} 19^{\text {hi }}$ regulatory subset has been specifically labeled as "B10 cells" because they are responsible for most IL-10 production by B cells and they appear to only produce IL-10 (69).

\subsubsection{Animal Models}

B10 cells were first characterized in studies of contact hypersensitivity (CHS) (68), a Th1 cell-mediated inflammatory reaction where B cells are not involved in Agpresentation and thereby have less of a role in augmenting inflammation (70).

Remarkably, CHS-induced inflammation is significantly exacerbated mice where all B cells were depleted using CD20 mAbs $(71,72)$. Thus, the absence of normal B cells augments Th1 cell-mediated inflammation, confirming a negative regulatory role for B cells during inflammation. The application of stimulation techniques that were known to induce $\mathrm{T}$ cells to produce cytokines with subsequent staining for intracellular IL-10 led to the identification of the B10 cell subset. Circulating IL-10-competent B cells were only found after Ag sensitization, with the percentage of B10 cells gradually decreasing after Ag challenge (68). A role for spleen IL-10-producing B10 cells in suppressing CHS responses in wild type mice was confirmed by the adoptive transfer of $C D 1 d^{\text {hi }} C D 5^{+} B$ cells purified from oxazolone-sensitized $\mathrm{CD} 20^{-/-}$mice into wild type recipient mice that had been depleted of B cells. Ag-sensitized CD1d ${ }^{\text {hi }} \mathrm{CD}^{+} \mathrm{B}$ cells restored normal CHS responses in B cell-depleted mice, while sensitized CD20 $0^{--}$non-B10 cells transferred into $B$ cell depleted mice did not reduce oxazolone-induced ear swelling. CD $1 \mathrm{~d}^{\text {hi }} \mathrm{CD} 5^{+} \mathrm{B}$ cells purified from unsensitized mice or mice sensitized with other Ags were without effect. 
Importantly, the adoptive transfer of IL- $10^{-/-} \mathrm{CD} 1 \mathrm{~d}^{\text {hi }} \mathrm{CD}^{+} \mathrm{B}$ cells did not affect CHS responses. Thus, B10 cells regulate T cell-mediated inflammatory responses in wild type mice through an IL-10-dependent mechanism.

B cells were shown over a decade ago to have regulatory properties during the induction of EAE (52), and genetically B cell-deficient mice develop a severe nonremitting form of EAE (55). Similarly, when B cells from wild type mice are depleted by CD20 mAb treatment seven days (d) before EAE induction, there is an increased influx or expansion of encephalitogenic T cells within the CNS, which significantly exacerbates disease symptoms (58). The adoptive transfer of $C D 1 d^{\text {hi }} C D 5^{+} B$ cells, but not other $B$ cells, normalizes EAE induced by CD20 mAb treatment on day -7. Furthermore, the adoptive transfer of CD1 $\mathrm{d}^{\text {hi }} \mathrm{CD} 5^{+} \mathrm{B}$ cells purified from $\mathrm{IL}-10^{-/-}$mice does not affect EAE severity, indicating that IL-10 production by B10 cells negatively regulates EAE initiation. Thereby, B10 cells normally counterbalance the positive effector roles of B cells during acute autoimmune responses, with B10 cell depletion increasing EAE severity (58). Further, B10 cells expand in mice after EAE induction, and the adoptive transfer of CD1 $\mathrm{d}^{\text {hi }} \mathrm{CD} 5^{+} \mathrm{B}$ cells from EAE-sensitized mice into wild type mice dramatically reduced EAE initiation, while the transfer of $C D 1 d^{\text {hi }} C D 5^{+} B$ cells from naïve donors only had modest effects (73). These results demonstrating the ability of Ag-specific B cells to regulate EAE through IL-10 production are confirmed by other studies $(55,74)$ that also demonstrate that toll-like receptor (TLR)-stimulated B cells secrete IL-10 and intact TLR signaling pathways and TLR-2 / 4 in B cells are required for recovery from EAE. Thereby, IL-10-producing regulatory B cells, most likely B10 cells, are important for controlling EAE severity and resolution.

IL-10-producing B cell subsets with varying phenotypes and origins also regulate intestinal inflammation during inflammatory bowel disease (63). Mizoguchi et al. showed that B cells suppress colitis in TCR $\alpha$ chain-deficient mice that develop chronic 
colitis, while B cells are not required for disease initiation (75). B cells themselves were the regulatory mediators and a B cell subset with up-regulated CD1d expression that appears during chronic inflammation suppresses the progression of intestinal inflammation (76). In addition, the adoptive transfer of mesenteric LN B cells also suppresses inflammatory bowel disease through a mechanism that correlated with an increase in regulatory $\mathrm{T}$ cell subsets (77). Thereby, IL-10-producing B cells can regulate immune-mediated gut inflammation.

B cells activated in vitro can also maintain tolerance and transfer protection from diabetes in NOD mice $(78,79)$, despite the pathogenic role that B cells play in disease initiation (51). The repeated transfusion of $1.2 \times 10^{7} \mathrm{BCR}$-stimulated NOD spleen B cells into NOD mice both delays the onset and reduces the incidence of diabetes in an IL-10dependent manner (78). The therapeutic effect of transfusing activated NOD B cells correlates with the polarization of $\mathrm{CD}^{+} \mathrm{T}$ cell responses toward a Th2 phenotype.

CIA is a T cell-dependent TD mouse model for rheumatoid arthritis in humans (80). B cells are important for initiating inflammation and arthritis since mature B cell depletion significantly reduces disease severity in mice, but does not inhibit established disease (43). By contrast, IL-10-producing B cell subsets with varying phenotypes and origins regulate inflammation during CIA. The in vitro culture and activation of splenic arthritogenic B cells with CD40 mAb and collagen results in B cell production of IL-10 but only low IFN $\gamma$ levels (81). When $5 \times 10^{6} \mathrm{CD} 40 \mathrm{mAb}$ - and collagen-activated B cells from the spleens of arthritogenic mice are injected intraperitoneally (i.p.) into recipient DBA / 1-T cell Ag receptor transgenic mice that are immunized on the same day with collagen, arthritis incidence ( $>50 \%$ reduction), disease severity $(>90 \%)$, and Th1 cell differentiation are inhibited in an IL-10-dependent manner. Further, the i.v. transfer of $4 \times 10^{5} \mathrm{CD} 21^{\text {hi }} \mathrm{CD}_{23}{ }^{+} \mathrm{IgM}^{+} \mathrm{B}$ cells from arthritogenic mice (remission phase) prevents CIA 
incidence and reduces disease severity (82). Thus, cells within this phenotypicallydefined subset can inhibit CIA progression.

A role for B cells in the development of tumor immunity has been assessed using mice given Friend murine leukemia virus gag-expressing mouse EL-4, D5 melanoma, or MCA304 sarcoma cells (83). In these studies, wild type mice are unable to control tumor progression, whereas EL-4 gag and D5 tumors (but not MCA304) were eliminated in $\mu \mathrm{MT}$ mice, which developed tumor-specific cytotoxic T lymphocytes after tumor challenge. Similarly, the growth of EL4 thymoma, MC38 colon carcinoma, and B16 melanoma was prevented or slowed in $\mu \mathrm{MT}$ mice in contrast to control mice (84). Increased $\mathrm{CD}^{+}$and $\mathrm{CD} 8^{+} \mathrm{T}$ cell responses to TS / A tumors are observed in $\mu \mathrm{MT}$ mice (85). Thus, these results suggest that B cells can also negatively regulate tumor immunity.

\subsubsection{Memory B Cell Contributions to Serum Ab Levels and Terminally-Differentiated Plasma Cell Numbers}

A hallmark of the adaptive humoral immune system is its ability to maintain an active memory of previous infection or Ag challenge in the form of long-lived Ag-specific $\mathrm{Ab}$ production and rapid memory recall responses (86). Ag-specific serum immunoglobulin (Ig) levels can be detected in hosts for decades after an infection or vaccination, and this phenomenon has been termed "serological memory." Serological memory is maintained by plasma cells, which secrete Ag-specific Ab, and possibly by memory B cells, which can rapidly differentiate into plasma cells upon stimulation. The mechanisms by which humoral memory and plasma cells are maintained remain in contention, and it is unclear whether the plasma cell pool is maintained independently of the memory B cell pool. As part of this debate, arguments have been made for both memory B cell-dependent mechanisms of serological memory maintenance, as well as for the existence of memory B cell-independent long-lived plasma cells (Figure 4). 

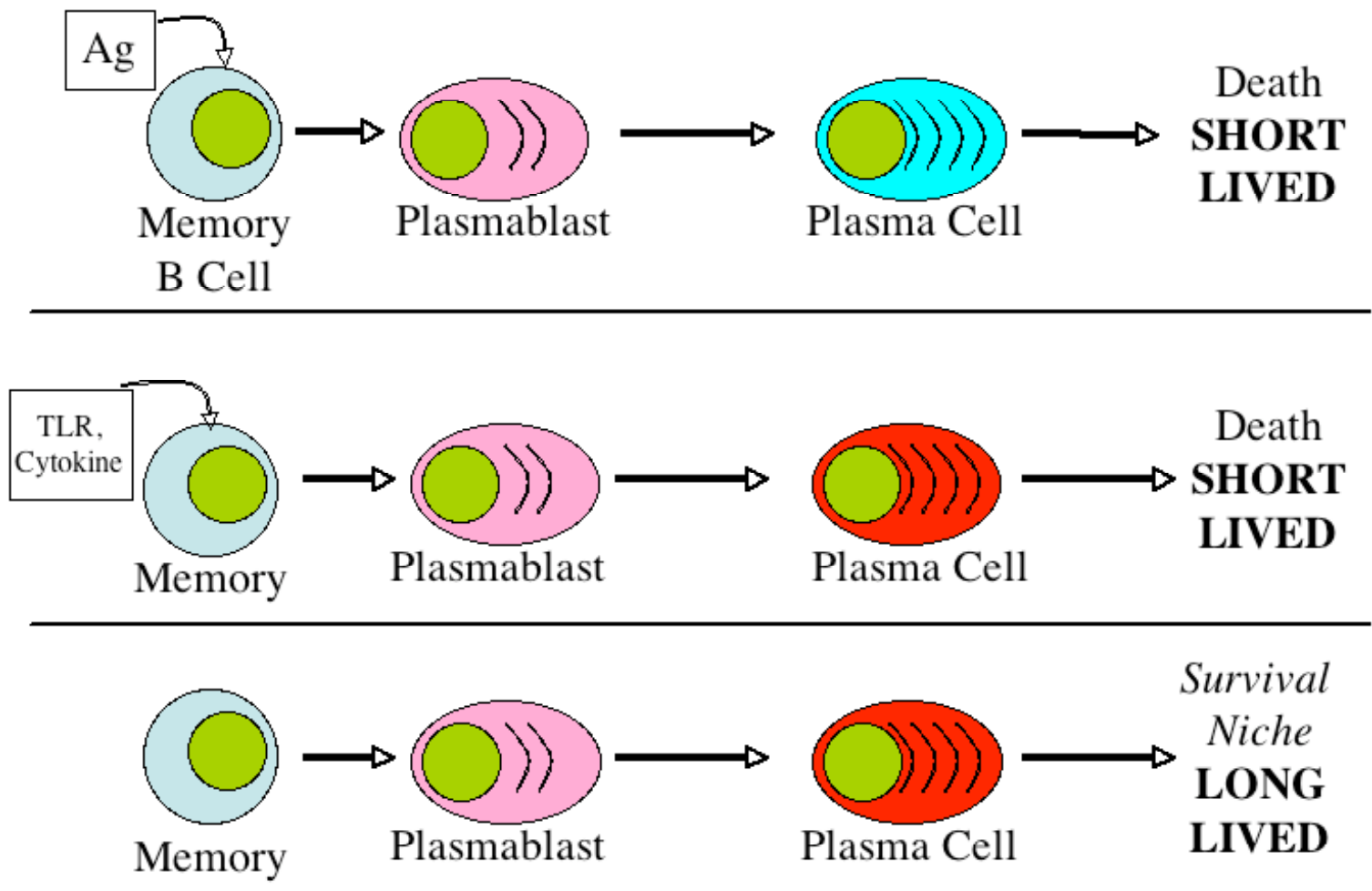

Figure 4. Mechanisms of serological memory maintenance. Memory B cell-dependent mechanisms of serological memory and plasma cell maintenance include Ag-dependent (top panel) and Ag independent (middle panel) mechanisms. Alternatively, plasma cells can be long-lived and survive in BM survival niches independently of contributions from memory B cells (bottom panel). 


\subsubsection{Memory B Cell-Dependent Models of Humoral Memory}

Memory B cell-dependent models of serological memory maintenance hinge upon memory B cells becoming reactivated in either Ag-specific or non-Ag-specific manners, proliferating, and differentiating into plasma cells that secrete Ag-specific Ab. One potential Ag-dependent mechanism involves the continuous reactivation of memory B cells by viruses that cause chronic infections (87). In this model, persisting viruses would be chronically presented to memory B cells, which would continue to proliferate and differentiate into virus-specific plasma cells with defined half-lives as long as the virus was present. In a similar model, memory B cells could potentially be chronically activated to differentiate into plasma cells by encountering cross-reactive environmental or self Ags. Repeated infection is an equally possible mechanism of serological memory maintenance. In this model, the host mounts a successful immune response to completely clear the infecting virus or pathogen, and both memory and plasma cells are generated. Subsequent infections with the same pathogen (or other pathogens expressing Ags similar to those of the primary pathogen) induce memory B cell proliferation and differentiation into Ab-secreting plasma cells with defined half-lives.

The classic model of serological memory maintenance is the persisting Ag model. In this model, Ag:Ab immune complexes bind to follicular DCs for extended lengths of time and chronically stimulate memory B cells to proliferate and differentiate into plasma cells. Ag can persist in lymphoid tissues for months after infection, thereby allowing for the perpetuation of the GC reaction (88-90). Thus, memory B cells may also be stimulated months after infection by this persisting Ag.

A final memory B cell-dependent model to explain the maintenance of serological memory involves the bystander polyclonal stimulation of memory B cells. Thereby, infection and inflammation in the host is thought to continually induce cytokine- or TLRmediated polyclonal activation and differentiation of memory B cells into plasma cells. 
For example, in vitro data suggests that memory B cells are competent to divide and differentiate into plasma cells upon stimulation with cytokines and TLR ligands (91). In vivo, booster vaccination with tetanus vaccine causes a 5 - to 10 -fold increase in the numbers of plasma cells specific for unrelated Ags, such as measles (92). Thus, memory $B$ cells can divide and differentiate in response to inflammatory signals such as TLR ligands and cytokines. However, whether these signals are required for the maintenance of serological memory is unknown.

\subsubsection{Long-Lived Plasma Cells}

In a memory B cell-independent model for the maintenance of serum Ab levels, primary and secondary immune responses generate a pool of long-lived plasma cells that occupy essential survival niches within the BM where they survive indefinitely, resulting in long-lived serum $\mathrm{Ab}$ levels $(7,12,86,93-95)$. Ab-secreting plasma cells generated in the spleen migrate to the BM where they can persist for months or years without the need for self-replenishment or turnover $(6,93,94,96-98)$. As further evidence of BM long-lived plasma cells, BM cell transfers confer long-lived Ab production, presumably due to the transfer of long-lived plasma cells (97). It has been shown that CXCR4 is important for plasma cell migration to the BM (86), while both BAFF and APRIL signals are important for the survival of long-lived plasma cells (99).

\subsection{Autoimmunity}

B cell development from stem cell to plasma cell is tightly controlled by multiple layers of regulation to produce a large and diverse repertoire of non-self-reactive B cells. To maintain tolerance to self, immature B cells in the BM that receive strong BCR signals after binding to free or cell-surface self Ag undergo apoptosis in a process called negative selection. Self-tolerance can also be maintained in the periphery, as self-reactive B cells here can be induced to change their specificity or be anergized or deleted. Similar 
mechanisms control T cell tolerance mechanisms as well. However, when central tolerance (negative selection in the BM) or peripheral tolerance mechanisms fail, autoreactive B and T cells can develop, leading to the development of autoimmune disease.

\subsubsection{Humoral and Cellular Immunity in Autoimmune Disease}

The development of normal immune responses and pathogenesis during autoimmunity requires both B cell-dependent humoral and TD cellular immune responses to work together to clear infection or cause tissue damage. It is wellestablished that $\mathrm{CD} 4^{+} \mathrm{T}$ cells function to promote $\mathrm{B}$ cell activation and $\mathrm{Ab}$-secreting plasma cell development. However, it is less clear whether and how B cells regulate cellular immunity and autoimmunity. Previous reports have provided conflicting results as to how B cells regulate immune responses. Some studies have shown that B cells do play a role in regulating cellular immune responses and autoimmunity $(16,17,19-21$, 27), while others have indicated that B cells play no role (22-26). These conflicting results may be due to the multitude of in vitro systems and in vivo animal models of disease and autoimmunity that have been used. Imperfect systems have also been used to assess the role of B cells in immune responses in vivo, as most previous studies have depleted B cells from birth with anti-IgM Ab or used genetically B cell deficient mice to assess the role of B cells in disease. Using these systems are not ideal, since many immune system abnormalities and defects have been noted in these mice. Further, it is clear that $\mathrm{Ab}$ produced by $\mathrm{B}$ cells plays an important role in the clearance of pathogens as well as in the pathogenesis of autoimmune disorders. However, the origin and maintenance of serological memory and plasma cell numbers remains unclear. Specifically, it is unknown whether memory B cells contribute to serological memory and 
whether the plasma cell pool is maintained and survives independently from memory B cells.

\subsubsection{B Cells in Autoimmune Disease}

As stated above, the mechanisms by which B cells contribute to normal immune responses and to autoimmune disease are unclear. The question remains: what role(s) do B cells contribute during normal and autoimmune immune responses? Specifically, do mature B cells positively regulate cellular immune responses and autoimmunity, do they negatively regulate cellular immune responses and autoimmunity, or can B cells perform both functions? Do mature and memory B cells contribute to the long-lived serum Ab and auto $\mathrm{Ab}$ levels that contribute to pathogen clearance and the pathogenesis of autoimmune disease?

Therefore, the goal of this dissertation is to determine what roles B cells play during normal immune responses and autoimmunity. In order to do this, we first assessed the function of B cells during tumor immunity as a model of immune system activation and cellular immunity in Chapter 3 of this dissertation. In Chapter 4 , we assessed the roles that B cells play during immune responses elicited by different allografts, since allograft rejection is thought to be largely T cell-mediated. Finally, in Chapter 5, we determined what mechanisms are responsible for the maintenance of the serum $\mathrm{Ab}$ levels that are responsible for protection against pathogens and tissue destruction during autoimmunity. 


\section{Materials and Methods}

\subsection{Mice}

Wild type C57BL/6, Rag1 $1^{-/-}\left(\mathrm{B} 6.129 S 7-\operatorname{Rag} 1^{\mathrm{tm1Mom}} / \mathrm{J}\right)$, B6.PL Thy1 ${ }^{\mathrm{a}}$ / Cy (B6.Thy1.1+), DBA/2, 129/X1, C57BL / 6-Tg(TcraTcrb)425Cbn/JB6 (OT-II), and C57BL / 6-Tg(TcraTcrb)1100Mjb / J (OT-I) mice were from Jackson Laboratory (Bar Harbor, ME). OT-II and OT-I transgenic mice generate $\mathrm{CD} 4^{+}$and $\mathrm{CD} 8^{+} \mathrm{T}$ cells that respond to peptides 323-339 and 257-264 of ovalbumin (OVA), respectively $(100,101)$. OT-II and OT-I mice (Thy1.2+ ${ }^{+}$were crossed to B6.Thy $1.1^{+}$mice to generate Thy1.1expressing T cells for adoptive transfer experiments. Hemizygous transgenic mice expressing human CD19 (TG-1 line) on a C57BL / 6 genetic background were as described $(102,103)$. In some experiments, mice received drinking water containing 5bromo-2-deoxyuridine (BrdU; $1 \mathrm{mg} / \mathrm{ml}$; Sigma-Aldrich, St. Louis, MO). Mice were housed in a pathogen-free barrier facility and were used at 6-12 weeks of age. These studies were approved by the Duke University Animal Care and Use Committee.

\subsection{Antibodies and Reagents}

Mouse CD20-specific mouse mAbs were as described (104). Other mAbs included: Fluorescein isothiocyanate (FITC)-, Phycoerythrin (PE)-, PE-Cy5-, allophycocyanin, or PE-Cy7-conjugated B220, CD1d (1B1), CD4 (H129.19), CD8 (536.7), CD21 (7G6), CD24 (M1/69), CD44 (IM7), CD138 (281-2), GL-7, Thy1.1 (OX-7), IFN- $\gamma(X M G 1.2)$, TNF $\alpha$ (MP6-XT22), and human CD19 (HIB19) mAbs from BD Pharmingen (San Diego, CA). L-selectin (CD62L; clone LAM1-116) mAb was as described (105). TCR $\beta$ chain mAb (H57-597) was from Ebioscience (San Diego, CA). Alexa Fluor 350-conjugated streptavidin was from Invitrogen-Molecular Probes (Carlsbad, CA). A BrdU Flow Kit (BD Pharmingen) was used to detect intracellular 
BrdU labeling. VLA-4 (clone PS / 2) and LFA-1 (clone M17 / 4) blocking mAbs were purified from hybridoma (ATCC, Manassas, VA) tissue culture supernatant fluid. Unlabeled and fluorescently conjugated Isotype-specific and anti-mouse Ig, IgG, or IgM secondary antibodies were from Southern Biotechnology Associates (Birmingham, AL). Functional grade CD3 (145-2C11) and CD28 (37.51) mAbs were from eBioscience (San Diego, CA). Rat anti-mouse $\mathrm{C} 4 \mathrm{~d} \mathrm{mAb}$, rat anti-mouse IgM $\mathrm{mAb}$, and horseradish peroxidase (HRP)-conjugated goat anti-Rat IgG polyclonal Ab were from Santa Cruz Biotechnology (Santa Cruz, CA). HRP-conjugated anti-mouse IgG was from Vector Laboratories (Burlingame, CA).

\subsection{Immunotherapy}

Sterile and endotoxin-free mouse CD20 (MB20-11), human CD19 (FMC63), and isotype-matched control mAbs were injected intravenously (i.v.) through lateral tail veins. Mice were given $250 \mu \mathrm{g}$ mAb in $200 \mu \mathrm{l}$ phosphate-buffered saline (PBS), unless otherwise indicated. For thioglycollate-induced peritonitis, $1 \mathrm{ml}$ of thioglycollate solution $(3 \% \mathrm{w} / \mathrm{v}$; Sigma-Aldrich) was injected i.p. $1 \mathrm{~d}$ before $\mathrm{mAb}$ treatment. In some cases, function-blocking LFA-1 and VLA-4 mAbs (200 $\mu$ g each) were injected i.p. as indicated.

\subsection{Immunizations}

Two-month (mo) old mice were immunized i.p. with trinitrophenyl (TNP)lipopolysaccharide (LPS) (50 $\mu$ g, Sigma-Aldrich) or dinitrophenyl (DNP)-Ficoll (25 $\mu$, Biosearch Technologies, San Rafael, CA) in $200 \mu$ PBS. Mice were also immunized i.p. with DNP conjugated to keyhole limpet hemocyanin (KLH) $(100 \mu \mathrm{g}$, CalbiochemNovabiochem Corp., La Jolla, CA) in $200 \mu \mathrm{l} \mathrm{CFA} \mathrm{(50 \%} \mathrm{v/v)} \mathrm{and} \mathrm{were} \mathrm{boosted} \mathrm{i.p.} 28 \mathrm{~d}$ later with DNP-KLH in $200 \mu \mathrm{l}$ IFA $(50 \% \mathrm{v} / \mathrm{v})$. In other experiments, mice were immunized i.p. with $100 \mu \mathrm{g}$ 4-hydroxy-3-nitrophenyl acetyl conjugated to chicken $\gamma$ - 
globulin (NP-CGG) precipitated in alum as described (8). Mice were bled before and after immunizations as indicated.

\subsection{Cell Preparation and Immunofluorescence Analysis}

Single-cell leukocyte suspensions from spleens and peripheral LNs (axillary, brachial, inguinal, and hilar) were generated by gentle dissection, and erythrocytes were hypotonically lysed. For multi-color immunofluorescence analysis, single cell suspensions $\left(10^{6}\right.$ cells) were stained at $4^{\circ} \mathrm{C}$ using predetermined optimal concentrations of $\mathrm{mAb}$ for $25 \mathrm{~min}$, as described (102). Cells with the forward and side light scatter properties of lymphocytes were analyzed using either a FACScan or LSR-II flow cytometer (Becton Dickinson; BD). Background staining was assessed using non-reactive, isotype-matched control mAbs (Invitrogen-Caltag, Carlsbad, CA). BM and spleen B cell subsets were gated as previously described (39).

Intracellular cytokine staining was performed using the Cytofix / Cytoperm kit (BD) according to the manufacturer's instructions. T cells were stimulated in vitro with plate-bound mAbs to CD3 $(1 \mu \mathrm{g} / \mathrm{ml})$ and CD28 $(10 \mu \mathrm{g} / \mathrm{ml})$ in the presence of Brefeldin A (1:1000 dilution; BD) for 3.5 hours (h) before surface staining and intracellular cytokine staining. In some experiments, $\mathrm{CD}^{-} 9^{+}$and $\mathrm{CD}^{-} 9^{-}$cells were separated using CD19 mAb-coated magnetic beads (Miltenyi Biotec, Auburn, CA).

\subsection{Immunohistology}

Frozen sections of spleen were fixed in acetone and stained with FITCconjugated GL-7, PE-conjugated TCR $\beta$ chain, and biotin-conjugated B220 mAbs followed by Alexa Fluor 350-labeled streptavidin at pre-determined optimal dilutions in saline containing $1 \%$ bovine serum albumin (BSA). Ab reactivity was visualized by fluorescence microscopy. 


\subsection{ELISAs}

DNP- and TNP-specific Ab levels in individual serum samples were determined in duplicate using an Ab isotype-specific enzyme-linked immunosorbent assay (ELISA) as described (103). Sera from TNP-LPS immunized mice were diluted 1:400, while sera from DNP-Ficoll and DNP-KLH immunized mice were diluted 1:1000 for analysis using ELISA plates coated with DNP-BSA (Calbiochem-Novabiochem Corp.) or TNP-BSA (Biosearch Technologies). Relative Ag-specific IgM and IgG titers were determined for each group of mice using pooled serum samples with results plotted as optical density (OD) versus dilution (log scale).

NP-specific serum Ab was detected by ELISA with IgG or IgG1 anti-NP Ab concentrations estimated by comparisons to standard curves generated using the high affinity NP-specific H33L $\gamma 1 \mathrm{mAb}$ (106). The relative affinities of serum NP-specific Ab responses were assessed by comparing the relative amounts of $\mathrm{Ab}$ bound to $\mathrm{NP}_{5}-\mathrm{BSA}$ versus $\mathrm{NP}_{25}$-BSA as described (8). Briefly, half of a 96-well ELISA plate was coated with $\mathrm{NP}_{5}-\mathrm{BSA}$ or $\mathrm{NP}_{25}$-BSA, and a set of sera was applied to the plate. OD values of $\mathrm{NP}_{5}$-specific and $\mathrm{NP}_{25}$-specific antibodies of each sample were used to calculate the relative ratio of NP-specific low- to high-affinity antibodies. Relative NP-specific IgM and IgG titers were determined for each group of mice using pooled serum samples with results plotted as OD versus dilution (log scale).

Serum auto Ab levels against single-stranded (ss) deoxyribonucleic acid (DNA) and histone were determined by ELISA using boiled calf thymus DNA (Sigma-Aldrich) or histone-coated microtiter plates (Sigma-Aldrich), respectively, as described (107)

\subsection{B Cell Adoptive Transfer Experiments}

Spleen B cells from mice immunized i.p. with $100 \mu \mathrm{g}$ of $\mathrm{NP}_{18}$-CGG in alum $42 \mathrm{~d}$ before harvest were enriched by $\mathrm{T}$ cell depletion using Thy1.2 mAb-coated magnetic 
beads (Invitrogen-Dynal, Carlsbad, CA, $>94 \%$ B220 ${ }^{+}$cells and $<0.7 \%$ Thy $1.2^{+}$cells). Spleen T cells from mice immunized i.p. with $100 \mu \mathrm{g}$ of CGG in alum $30 \mathrm{~d}$ before harvest were enriched by B cell depletion using B220 mAb-coated magnetic beads (InvitrogenDynal, $<0.8 \%$ B220 ${ }^{+}$cells $)$. Equal numbers of B and T cells $\left(3 \times 10^{7}\right)$ were injected i.v. into $\operatorname{Rag} 1^{-/-}$mice immediately before i.v. injection of MB20-11 or isotype-matched control mAbs. One d after mAb treatment, all mice were given soluble $\mathrm{NP}_{18}$-CGG (100 $\mu g)$ i.v., with sera collected at the times indicated. For enzyme-linked immunospot (ELISPOT) assays, BM and spleen cells were harvested from individual mice $10 \mathrm{~d}$ after boost immunizations.

\subsection{ELISPOT Assays}

The frequency of NP-specific Ab-secreting cells (ASCs) from single-cell splenocyte and $\mathrm{BM}$ suspensions were estimated by ELISPOT assays using $\mathrm{NP}_{6}^{-}, \mathrm{NP}_{25^{-}}$, or $\mathrm{NP}_{33}$-BSA conjugates as described (8). BM and spleen cells were added to ImmobilonP Multiscreen 96-well plates (Millipore, Billerica, MA) that were pre-coated with NPBSA $(5 \mu \mathrm{g} / \mathrm{ml})$ at either $10^{4}, 10^{5}$, or $10^{6}$ cells per well in culture medium (100 $\mu \mathrm{l}$; Roswell Park Memorial Institute (RPMI) 1640 medium containing 10\% FCS, 10 mM glutamine, $100 \mathrm{U} / \mathrm{ml}$ penicillin/streptomycin, and $55 \mu \mathrm{M}$ 2-ME). After incubating the plates for $3 \mathrm{~h}$ at $37^{\circ} \mathrm{C}$ in a humidified $\mathrm{CO}_{2}$ incubator, the plates were washed 3 times and incubated with alkaline phosphatase-conjugated polyclonal goat anti-mouse IgG or IgG1 antibodies (Southern Biotechnology Associates) for $1 \mathrm{~h}$ at room temperature. After washing, the plates were developed using BCIP / NBT substrate (Sigma-Aldrich). Similarly, the total number of splenic and BM IgG and IgM ASCs was determined using plates coated with polyclonal anti-mouse Ig and developed with alkaline phosphatase-conjugated goat anti-mouse IgM or IgG antibodies (Southern Biotechnology Associates). For sorting of cell populations before use in ELISPOT assays, the MACS system (Miltenyi Biotech, 
Auburn, CA) was used to purify CD20+ and CD20- cells. Cells labeled with biotinylated MB20-11 mAb and anti-biotin microbeads were used to positively select CD20 ${ }^{+}$cells. Flow-through cells were harvested and run a second time over the magnetic column to remove contaminating $\mathrm{CD}_{20} 0^{+}$cells. The purity of the $\mathrm{CD}^{-} 0^{-}$population was $>98 \%$, while CD20 ${ }^{+}$cell purity was $>95 \%$.

\subsection{B Cell CFSE Labeling and Cell Culture.}

Purified B cells were differentiated in culture by stimulation with LPS or CD40 mAb plus IL-4 as described (108). Briefly, T cell-depleted splenocytes (>93\% B220 $)$ were labeled with $1 \mu \mathrm{M}$ Carboxyfluorescein Succinimidyl Ester (CFSE; CFSE Vybrant ${ }^{\mathrm{TM}}$, Invitrogen-Molecular Probes) according to the manufacturer's instructions. B cells were cultured in 24-well flat-bottom tissue culture plates $\left(3 \times 10^{6}\right.$ cells in $1.5 \mathrm{ml}$ of culture medium/well) at $37^{\circ} \mathrm{C}$ with $5 \% \mathrm{CO}_{2}$ in the presence of LPS $(10 \mu \mathrm{g} / \mathrm{ml})$ or CD40 mAb (1 $\mu \mathrm{g} / \mathrm{ml}$. HM40-3, BD) in combination with IL-4 (100 U/ml, Sigma-Aldrich) for $72 \mathrm{~h}$. CD20 expression levels were assessed by immunofluorescence staining of harvested cells with flow cytometry analysis.

\subsection{Cell Lines and Tumor Models}

The P815 and B16/F10 cell lines were purchased from ATCC (Manassas, VA) and were cultured in Dulbecco's modified eagle medium containing 10\% FCS, $200 \mathrm{mg} / \mathrm{ml}$ penicillin, $200 \mathrm{U} / \mathrm{ml}$ streptomycin, $4 \mathrm{mM}$ L-Glutamine, and $50 \mathrm{mM} \beta$-mercaptoethanol (all from Invitrogen, Carlsbad, CA). The AG8 cell line (ATCC) was cultured in RPMI 1640 media containing the same supplements used for culturing P815 and B16 / F10 cells. The OVA-secreting B16 / F0 / OVA cell line (109) was kindly provided by Dr. Edith Lord (Univ. Rochester, Rochester, NY). A stable B16 / F10 cell line expressing membranebound OVA (B16/F10/mOVA) was produced using an expression plasmid (pIRES2EGFP) containing cDNA encoding full-length OVA protein linked to the transmembrane 
region of $H-2 D^{b}$ (110), which was generously provided by Dr. Marc Jenkins (Univ. Minnesota, Minneapolis, MN). Cells expressing green fluorescence protein (GFP) at high levels were selected by multiple rounds of fluorescence-based cell sorting. Cells were passaged minimally and maintained in complete Dulbecco's modified eagle medium containing 10\% FCS, $200 \mathrm{mg} / \mathrm{ml}$ penicillin, $200 \mathrm{U} / \mathrm{ml}$ streptomycin, $4 \mathrm{mM}$ L-Glutamine, and $50 \mathrm{mM}$ ß-mercaptoethanol (all from Invitrogen-Gibco, Carlsbad, CA). To maintain OVA expression, B16/F0/OVA and B16/F10/mOVA cell cultures contained G418 $(400 \mu \mathrm{g} / \mathrm{ml})$.

In the cutaneous melanoma tumor model, anesthetized mice were injected subcutaneously (s.c.) on the shaved right lateral flank with either $1 \times 10^{5}$ or $1.5 \times 10^{6}$ B16 / F10, B16/F10/mOVA, or B16/F0 / OVA tumor cells in $200 \mu l$ of sterile PBS.

Tumor volumes were monitored and calculated using the equation: $\mathrm{V}=4 \pi\left(\mathrm{L}_{1} \times \mathrm{L}_{2}{ }^{2}\right) / 3$, where $\mathrm{V}=$ volume $\left(\mathrm{mm}^{3}\right), \mathrm{L}_{1}=$ the longest radius $(\mathrm{mm})$, and $\mathrm{L}_{2}=$ the shortest radius (mm). In the lung metastasis model, between $4 \times 10^{4}$ and $5 \times 10^{5}$ B16/F10 or B16 / F0 / OVA tumor cells in $250 \mu$ l of sterile PBS were injected i.v. through lateral tail veins. At predetermined time points, lungs were removed from euthanized mice. The numbers of metastasis foci were counted visually in a blinded fashion using a stereomicroscope.

\subsection{Measurement of Tumor-Specific Ab Production}

Serum $\mathrm{Ab}$ generated in response to tumors was evaluated using an indirect immunofluorescence assay. Sera from control or tumor-bearing mice were diluted 1:16 and incubated with B16/F0/OVA cells for $30 \mathrm{~min}$ at $4^{\circ} \mathrm{C}$. The cells were then washed extensively, incubated with fluorochrome-conjugated polyclonal anti-mouse IgG and IgM antibodies, and analyzed by flow cytometry on a FACScan flow cytometer (BD). 
Background staining mean fluorescence intensity $(\mathrm{MFI})$ values $(<10)$ were subtracted from the experimental values.

\subsection{T Cell Adoptive Transfer Experiments}

Donor Thy $1.1^{+}$OT-II or OT-I T cells from pooled spleens and LNs were enriched with $\mathrm{CD}^{+}$and $\mathrm{CD} 8^{+} \mathrm{T}$ cell isolation kits (Miltenyi Biotec), respectively, and labeled with CFSE Vybrant ${ }^{\mathrm{TM}}$ CFDA SE fluorescent dye $(1 \mu \mathrm{M}$; Invitrogen-Molecular Probes, Carlsbad, CA) according to the manufacturer's instructions. $2.5 \times 10^{6}$ labeled Thy $1.1^{+}$ cells were administered i.v. to Thy $1.2^{+}$congenic recipients $1 \mathrm{~d}$ after tumor transfer. The proliferation of transferred cells was visualized by flow cytometry analysis of CFSElabeled Thy $1.1^{+}$cells. Transferred OT-II CD4 ${ }^{+}$or OT-I CD8 ${ }^{+} \mathrm{T}$ cells were identified by Thy1.1 and CD4 or CD8 mAb staining, respectively.

\subsection{Mouse Cardiac Transplantation}

Heterotopic murine cardiac transplants were as described (111). Recipient C57BL / 6-129 (H-2b) mice were anesthetized using isoflurane and prepared by separating the vena cava and aorta between the renal vasculature and the iliac bifurcation. The donor heart was dissected from a fully MHC-mismatched (DBA / 2; H2d) mouse, and an end-to-side anastomosis was created between the recipient aorta and the donor heart ascending aorta. A similar vascular anastomosis was created between the donor superior vena cava and the inferior vena cava of the recipient. The total ischemic time averaged $15 \mathrm{~min}$ and was not different between the groups. Surgical mortality of the recipients was less than $10 \%$. Allograft survival was determined by directly palpating the cardiac impulse through the abdominal wall, with graft failure defined as the cessation of a palpable heartbeat. 


\subsection{Measurement of Allograft-Specific Ab Production}

Serum $\mathrm{Ab}$ generated in response to allografting was evaluated using cell lines and indirect immunofluorescence staining assays.. Sera from mice were diluted (1:40) and incubated with P815 cells (for DBA / 2 mice) or AG8 cells (for BALB / C mice) for 30 min at $4^{\circ} \mathrm{C}$. The cells were then washed three times, incubated with fluorochrome-conjugated secondary polyclonal anti-mouse IgG or IgM Abs, and analyzed by flow cytometry on a FACScan flow cytometer (BD). Staining obtained using secondary Ab alone was considered background, with these MFI values $(<10)$ subtracted from the experimental values.

\subsection{Skin Grafting}

The skin graft procedures were modified from the methods of Billingham (112). Briefly, dorsal skin was dissected from donor mice after removal of hair with electric clippers and cleansing with $70 \%$ ethanol. Subcutaneous fascia was gently removed from the undersurface of donor skin with a Number 15 scalpel. The prepared donor skin was then stored at $4^{\circ} \mathrm{C}$ on PBS-soaked gauze in sterile petri dishes until use (generally within $90 \mathrm{~min}$ ). Recipient mice were anesthetized with pentobarbital administered i.p. at a dose of $0.7 \mathrm{mg} / \mathrm{g}$ body weight. Hair was removed from the dorsolateral skin of recipient mice, and skin was cleansed with $70 \%$ ethanol and painted with flexible collodian. Eightmillimeter-diameter graft beds were prepared by removing the epidermis and superficial dermis leaving the fascia layer intact. Skin grafts were then placed by trimming donor skin to the graft beds. Grafts were dressed with petroleum-coated Telfa gauze held in place with circumferential bandages. Dressings were removed on day 6 after graft placement. Mice were monitored daily following surgery. Duplicate grafts were placed on each mouse, and syngeneic skin grafts served as controls for non-specific 
inflammation related to surgery. Grafts were considered to be rejected at the time of sloughing or upon complete conversion to a hard avascular eschar.

\subsection{Kidney Transplantation}

Vascularized kidney transplants were as described (113). Mice were anesthetized with isoflurane, and the donor kidney, ureter, and bladder were harvested en bloc, including the renal artery with a small aortic cuff and the renal vein with a small vena caval cuff. These vascular cuffs were anastomosed to the recipient abdominal aorta and vena cava, respectively, below the level of the native renal vessels. Donor and recipient bladders were attached dome to dome. The right native kidney was removed during transplant, and the left native kidney was removed through a flank incision 1-3 d later. The adrenal glands and their blood supply were preserved intact. Mice surviving $100 \mathrm{~d}$ were sacrificed with $100 \mathrm{~d}$ survival used when calculating mean survival times. These mice were considered to be survivors in the Log-Rank statistical analysis.

\subsection{Kidney Histopathologic and Immunohistology Studies}

A portion of the transplanted kidneys was placed in buffered $10 \%$ formalin, sectioned, and stained with Hematoxylin and Eosin (H\&E), Periodic Acid Schiff (PAS), and Masson's Trichrome. All tissues were examined by a pathologist masked to the experimental groups. The overall severity of rejection was determined by the pattern and intensity of inflammatory cell infiltrates in both glomeruli and tubulointerstitial areas, and glomerular, tubular, and vascular abnormalities. Grading was performed using a semiquantitative scale in which 0 represented the absence of histologic abnormalities, and $1+, 2+, 3+$, and $4+$ represented mild, moderate, moderately severe, and severe abnormalities, respectively, as described $(114,115)$. An overall histologic score was obtained for each kidney by summing the individual grades for the glomeruli, tubules, interstitium, and vasculature (115). 
To assess C4d, IgG, and IgM deposition, a portion of transplanted kidneys was removed, embedded in optimal cutting temperature (OCT) compound, and snap frozen in pre-cooled 2-methylbutane. Tissue sections $(5 \mathrm{~mm})$ were cut with a cryostat, air dried, fixed in acetone, washed with PBS ( $\mathrm{pH} 7.4$ ), blocked with normal goat serum, and stained with the appropriately diluted primary $\mathrm{mAb}$. Primary Ab binding was detected with a species-specific HRP-conjugated secondary Ab followed by development using 3,3' Diaminobenzidine as the substrate (Vector Laboratories). Grading of the C4d, IgG, and IgM staining intensities of peritubular capillaries was performed independently by blinded pathologists using a semiquantitative scale, in which 0 represented no staining, and $1+, 2+$, and $3+$ represented mild, moderate, and severe staining, respectively.

\subsection{Statistical Analysis}

All data are shown as means \pm the standard error of the mean (SEM). The Student's t-test was used to determine the significance of differences between sample means. Significance in survival studies was determined using the Log-Rank test. 


\section{B Cells Are Required for Optimal CD4 ${ }^{+}$and $\mathrm{CD} 8^{+} \mathrm{T}$ Cell Tumor Immunity: Therapeutic B Cell Depletion Enhances B16 Melanoma Growth in Mice}

The following text was slightly modified from its original manuscript, "B Cells Are Required for Optimal CD4 ${ }^{+}$and $\mathrm{CD}^{+} \mathrm{T}$ Cell Tumor Immunity: Therapeutic B Cell Depletion Enhances B16 Melanoma Growth in Mice," published in The Journal of Immunology in 2010.

\subsection{Introduction}

B lymphocytes are the effector cells of humoral immunity that terminally differentiate into Ab-secreting plasma cells. However, B cells have multiple other functions that either positively or negatively influence cellular immunity. For example, B cells can positively regulate cellular immune responses by serving as APCs and / or by providing costimulatory signals to T cells $(20,27)$. Regulatory B cells (B10 cells) have also been identified that negatively regulate inflammation and immune responses through the production of IL-10 $(58,68,116)$. Conflicting positive and negative roles for B cells during tumor immunity have also been reported. Ag presenting B cells can induce tumor-specific cytotoxic T cell activation (117). In addition, mice depleted of B cells since birth with anti-IgM serum do not develop fully protective T cell immunity to virusinduced tumors $(118,119)$. B cell $\mathrm{Ab}$ responses are thought to contribute modestly, if at all, to tumor immunity $(84,85,120)$, while $\mathrm{Ab}$ production may contribute to chronic inflammation that enhances tumor development (121-123).

Negative regulatory functions for B cells during immune responses to tumors have also been proposed. Treatment of mice from birth with anti-IgM serum to deplete B cells increases their resistance to fibrosarcoma growth and lowers the incidence of metastasis (124). Mammary carcinoma invasion and metastasis are also reduced by $70 \%$ in mice 
subsequently treated with anti-IgM/IgG Ab (125). Tumors also grow more slowly and are rejected more frequently in severe combined immunodeficient mice given only $\mathrm{T}$ cells in contrast to mice reconstituted with both $\mathrm{T}$ and B cells (126). Other studies have predominantly used genetically B cell-deficient $\mu \mathrm{MT}$ mice, where B cell deficiency enhances $\mathrm{CD}^{+} \mathrm{T}$ cell priming and help for $\mathrm{CD}^{+} \mathrm{T}$ cell-mediated tumor immunity (85). B16 melanoma, EL4 thymoma, and MC38 colon carcinoma growth is also slowed in $\mu$ MT mice (84). Similarly, anti-tumor immune responses to EL-4 thymoma and D5 melanoma are enhanced in $\mu \mathrm{MT}$ mice, potentially due to the absence of IL-10-producing B cells (83). B cell depletion in humans using rituximab, a chimeric anti-human CD20 $\mathrm{mAb}$, may also delay or suppress advanced colon cancer progression and metastases (125). These results have led to the prediction that B cell depletion could therapeutically enhance immune responses to tumors. However, most of these studies were performed using mice in which the immune system develops in the complete absence of B cells. Because of this, $\mu \mathrm{MT}$ mice have severe immune system abnormalities (34). For example, $\mathrm{T}$ cell repertoire and numbers are decreased significantly in $\mu \mathrm{MT}$ mice $(34,127)$. In addition, B cells help to organize lymphoid organ architecture, so the spleens of $\mu \mathrm{MT}$ mice are smaller in size (127) and lack follicular DCs and several macrophage populations (37). Moreover, DCs produce enhanced levels of IL-12 in $\mu$ MT mice, thus skewing immunity towards Th1 responses (23). Therefore, conclusively understanding the role of B cells in tumor immunity has been difficult. Furthermore, B cells may have different functions during immune responses to different tumors, much as B cells have reciprocal-regulatory functions during the development of autoimmunity $(27,58,67,68$, 128).

The role of B cells during tumor immunity in wild type mice with intact immune systems was examined in this study using a mAb specific for CD20 that selectively 
depletes B cells in vivo by monocyte-mediated Ab-dependent cellular cytotoxicity (71). More than $95 \%$ of mature B cells in the blood and primary lymphoid organs are depleted after two d by a single dose of MB20-11 CD20 mAb (250 $\mu$ g per mouse), with the effect lasting up to eight weeks (39). To determine the role of B cells in tumor immunity, two physiologically relevant and well-characterized melanoma cell lines were used that were derived from a spontaneously-arising C57BL / 6 melanoma $(109,129)$. B16 / F10 cells were derived from the B16/F0 line after 10 successive in vivo passages $(109,130)$. These poorly-immunogenic tumor lines express low MHC class I levels and do not express MHC class II molecules, but both molecules are inducible upon IFN- $\gamma$ exposure (131). B16 / F10 cells are highly aggressive and metastatic, while B16 / F0 cells metastasize less and are less aggressive $(130,132)$. Using these cells, B cell depletion in mice with otherwise intact immune systems was found to significantly accelerate melanoma growth and metastasis, and reduce the induction of $\mathrm{CD} 4^{+}$and $\mathrm{CD} 8^{+}$effector-memory and cytokine-secreting T cells. Thus, B cells are required for optimal T cell activation during this model of tumor immunity.

\subsection{Results}

\subsubsection{B Cell Depletion Rnhances B16 Melanoma Growth and Metastasis}

B cell contributions to non-lymphoid tumor immunity were assessed in adult wild type mice with otherwise intact immune systems following B cell depletion using CD20 mAb. Circulating, spleen, and LN B cell numbers are reduced $>95 \%$ by day 2 in C57BL / 6 mice, and begin to recover by day 57 following MB20-11 CD20 mAb treatment (250 mg/mouse, ref. 39, 71, 133). B16/ F10 melanoma growth and metastasis were assessed in littermate mice given either control or CD20 mAb $7 \mathrm{~d}$ before the i.v. transfer of $3 \times 10^{5}$ B16/F10 melanoma cells. In this model of aggressive melanoma 
metastasis, tumor cells preferentially localize to the lungs where they appear as distinct pigmented foci on the lung surface (134).

Two-fold more tumor foci were observed on the lungs of CD20 mAb-treated mice compared to control mAb-treated mice by 7 and $14 \mathrm{~d}$ following tumor injection $(\mathrm{p} \leq 0.006$, Figure 5A). Individual tumor foci were also larger and covered a greater surface area in B cell-depleted mice. Similar results were obtained when B cell-depleted mice were given $1.5 \times 10^{6} \mathrm{~B} 16 / \mathrm{F} 10$ cells s.c. Tumor volumes at the site of injection were two-fold greater in B cell-depleted mice when compared with control mAb-treated mice $14 \mathrm{~d}$ after B16 / F10 cell transfers ( $\mathrm{p} \leq 0.005$, Figure 5B). When lower doses of B16 / F10 cells were used, similar increases in s.c. tumor volume and lung tumor foci surface area were noted, while there was only a modest increase the in number of lung foci in CD20 mAb-treated mice (Figure 5C-D). Thus, B cell depletion may not directly affect tumor metastasis, but allows for increased growth of implanted tumor cells. However, increased tumor growth did not significantly affect the survival of CD20 mAb-treated mice that received i.v. injections of either low (Figure 5E) or high (Figure 5F) doses of B16/F10 cells.

B cell depletion also facilitated the growth and metastasis of the B16 / F0 / OVA cell line, a substantially less aggressive tumor derived from the B16 / F0 cell line. The frequency of detectable melanoma lesions in littermates given $5 \times 10^{5}$ B16 / F0 / OVA cells was significantly increased in the absence of B cells by day 28 ( $p=0.01$; Figure 5G). CD20 mAb treatment also significantly enhanced the growth of s.c. tumors 7 and $14 \mathrm{~d}$ after littermates were given $1.5 \times 10^{6}$ B16/F0 / OVA cells $(\mathrm{p}=0.005$, Figure 5H). Thus, B cell depletion during tumor challenge enhanced the growth of both B16/F10 and B16 / F0 / OVA tumors.

To determine whether OVA expression affects B16 tumor growth, B16/F10 cells were stably transfected to express membrane-bound OVA (B16/F10/mOVA), and 
compared to wild type B16/F10 cells. OVA expression by both the B16/F0/OVA and B16/F10/mOVA cells was sufficient to stimulate the proliferation of CD8 ${ }^{+}$OVA peptide-specific OT-I cells in vivo (Figure 5I). Although both the wild type B16 / F10 and B16 / F10 / mOVA cells grew similarly in control mAb-treated mice, both cell lines generated tumors that were twice as voluminous in CD20 mAb-treated mice (Figure 5J). Thus, OVA expression by B16 / F10 tumors did not affect tumor growth. Since all tumors responded similarly to B cell depletion and both the B16/F10/mOVA and B16 / F0 / OVA cells stimulated OT-I cells equally in vivo, the less aggressive and wellcharacterized B16/F0 / OVA cells were utilized for all subsequent studies. 
A
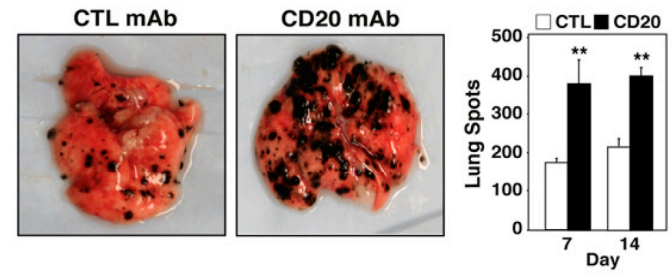

C

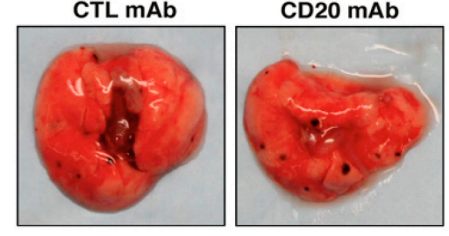

E

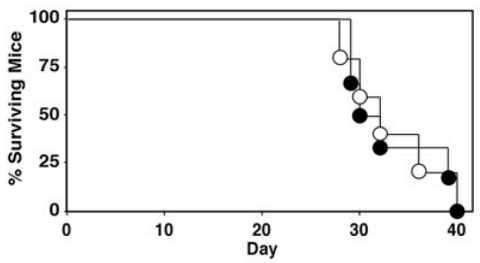

G
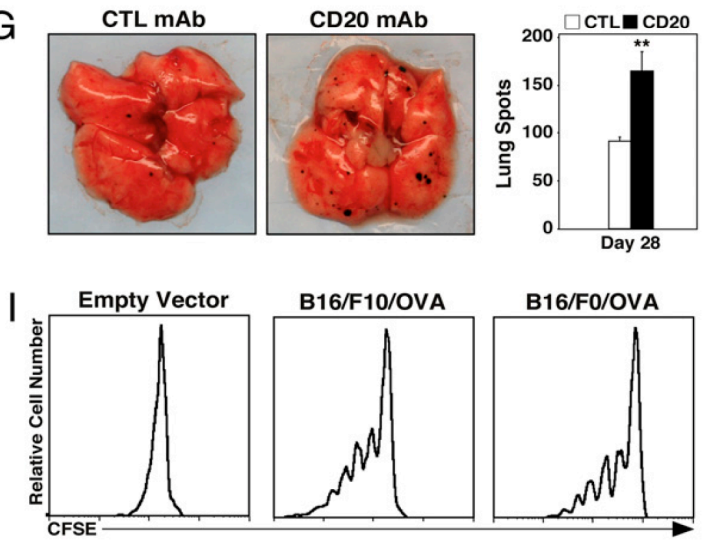
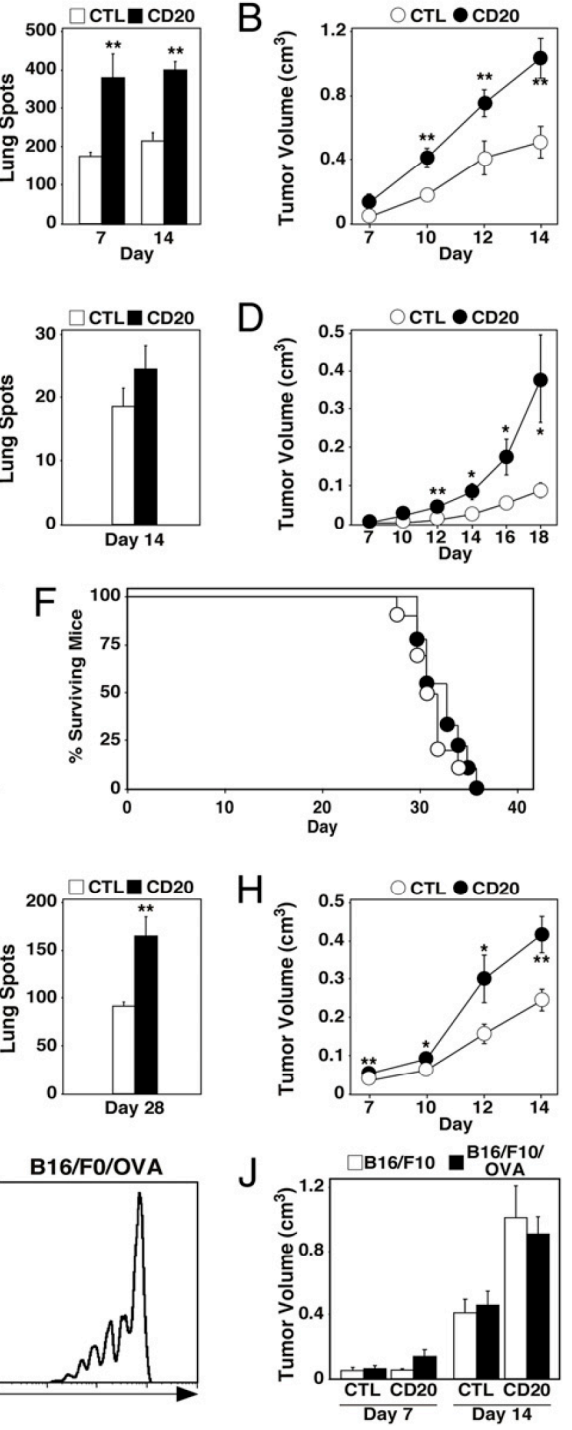

Figure 5: B cells enhance tumor immunity to B16 melanoma. (A, C, G) B cell depletion enhances B16 melanoma metastasis. Mice were given CD20 or control (CTL) mAb $7 \mathrm{~d}$ before i.v. transfer of (A) $3 \times 10^{5}$ B16/F10, (C) $5 \times 10^{4}$ B16/F10, or (G) $3 \times 10^{5}$ B16 / F0 / OVA cells. Representative lungs from control mAb- and CD20 mAb-treated mice $14 \mathrm{~d}$ (B16/F10 high dose), $14 \mathrm{~d}$ (B16/F10 low dose), or $28 \mathrm{~d}$ (B16/F0/OVA) after tumor transfer are shown. Histograms represent mean $( \pm$ SEM) numbers of visible pigmented tumor foci on the surface of lungs on the indicated days after transfer $(n \geq 5$ mice for each group). (B, D, H) B cell depletion enhances B16 melanoma growth. Mice were given $\mathrm{CD} 20$ or control mAb $7 \mathrm{~d}$ before s.c. transfer of (B) $1.5 \times 10^{6} \mathrm{~B} 16 / \mathrm{F} 10$, (D) 1 $\times 10^{5} \mathrm{~B} 16 / \mathrm{F} 10$, or $(\mathrm{H}) 1.5 \times 10^{6} \mathrm{~B} 16 / \mathrm{F} 0 /$ OVA cells. Values represent mean tumor volumes $( \pm$ SEM) at the site of transfer on the indicated days ( $\mathrm{n} \geq 10$ for each group). (EF) Mouse survival following the i.v. transfer of (E) $4 \times 10^{4}$ or (F) $3 \times 10^{5}$ B16/ F10 cells on day 0. All mice were given either control (open circles) or CD20 (closed circles) mAb on day -7 ( $\mathrm{n} \geq 6$ mice for each group). (I) B16/F10/mOVA cells activate OVA peptidespecific CD ${ }^{+} \mathrm{T}$ cells. Mice were given $3 \times 10^{5}$ i.v. B16/F10 cells transfected with an 
Figure 5 (Continued) empty vector (Empty Vector, n=1), B16 / F10 cells transfected to express OVA (B16/F10/mOVA, $n=3)$, or B16/F0/OVA $(n=3)$ cells 1 d before CFSElabeled Thy $1.1^{+} \mathrm{CD} 8^{+} \mathrm{T}$ cells from OT-I mice were transferred into each mouse. Lungdraining hilar LN lymphocytes were isolated $5 \mathrm{~d}$ later, with CFSE expression and dilution by CD ${ }^{+}$Thy $1.1^{+} \mathrm{T}$ cells assessed by flow cytometry. Representative histograms showing CFSE intensity of CD ${ }^{+}$Thy $1.1^{+}$cells are shown. (J) B16/F10 and

$\mathrm{B} 16 / \mathrm{F} 10 / \mathrm{mOV}$ A have similar growth characteristics. Mice were given CD20 or control $\mathrm{mAb} 7 \mathrm{~d}$ before s.c. transfer of $1.5 \times 10^{6} \mathrm{~B} 16 / \mathrm{F} 10$ or B16/F10/mOVA cells. Values represent mean tumor volumes $( \pm$ SEM) at the site of transfer on the indicated days ( $\mathrm{n} \geq 10$ for each group). Significant differences between means for the same days are indicated: ${ }^{*}, \mathrm{p}<0.05 ;{ }^{* *}, \mathrm{p}<0.01$. 


\subsubsection{CD20 mAb Rffectively Depletes B Cells in Tumor-Bearing Mice}

Whether B16/F0 / OVA cells affect CD20 mAb-induced B cell depletion was assessed in tumor-free mice and mice receiving $1.5 \times 10^{6}$ B16/F0/OVA cells $7 \mathrm{~d}$ after control or CD20 mAb treatment. Fourteen d after tumor transfers, all mice had $>0.15$ $\mathrm{cm}^{3}$ s.c. tumors. The numbers of $\mathrm{B} 220^{+} \mathrm{B}$ cells within LNs and spleens was assessed by immunofluorescence staining with flow cytometry analysis. B220 $0^{+}$cell numbers in tumordraining LNs increased by 7.3-fold in tumor-bearing mice when compared with littermates without tumors ( $\mathrm{p}<0.02$; Figure $6 \mathrm{~A}-\mathrm{C})$. Nonetheless, CD20 mAb treatment depleted $>99 \%$ of LN and spleen B cells in mice with or without tumors. Thereby, enhanced B16/F0/OVA growth in CD20 mAb-treated mice did not result from reduced B cell depletion.

\subsubsection{Anti-tumor Ab responses in B16/F0/OVA-bearing mice}

Although B cell depletion abrogates most subsequent humoral immune responses to model Ags (2), the effects of CD20 mAb treatment on anti-tumor Ab responses is unknown. Therefore, mice were given control or CD20 mAb $7 \mathrm{~d}$ before s.c. transfer of B16 / F0 / OVA cells. Fourteen d later, OVA-specific IgM or IgG Ab responses were not detected in the serum of tumor-bearing mice by ELISA (data not shown). Therefore, B16 / F0 / OVA cells were incubated with diluted serum, washed, stained with fluorescently labeled anti-IgM or -IgG antibodies, and analyzed by flow cytometry. No significant differences in tumor-reactive $\mathrm{Ab}$ levels were noted between control and $\mathrm{B}$ celldepleted mice (Figure 6D). Most control or tumor-bearing mice had similar low levels of B16 / F0 / OVA-reactive IgM and IgG antibodies regardless of CD20 mAb treatment. However, a small proportion of control and tumor-bearing mice had high titers of B16 / F0 / OVA-reactive IgM and IgG antibodies. As previously described, mice with 
significant $\mathrm{B} 16$-reactive $\mathrm{Ab}$ responses are likely to have previously produced IgM and IgG in response to viral Ags that are also expressed by B16 / F0 / OVA cells (135). Thus, C57BL / 6 mice do not generate detectable B16/F0/OVA-specific Ab responses, consistent with previous observations $(109,136)$. 


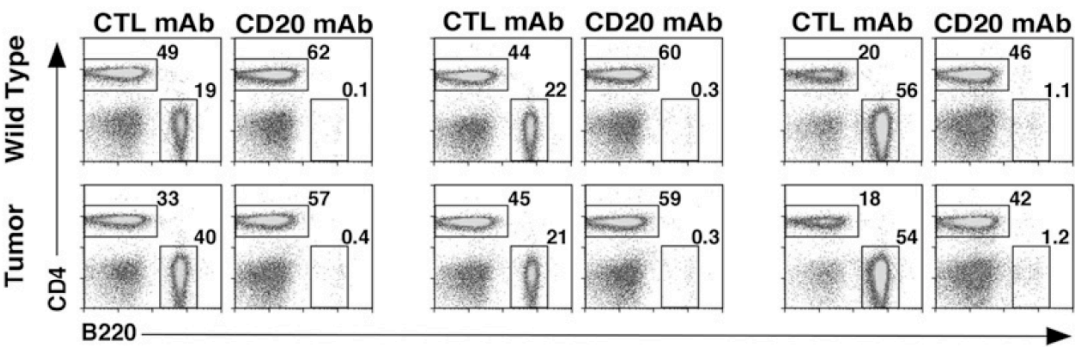

B
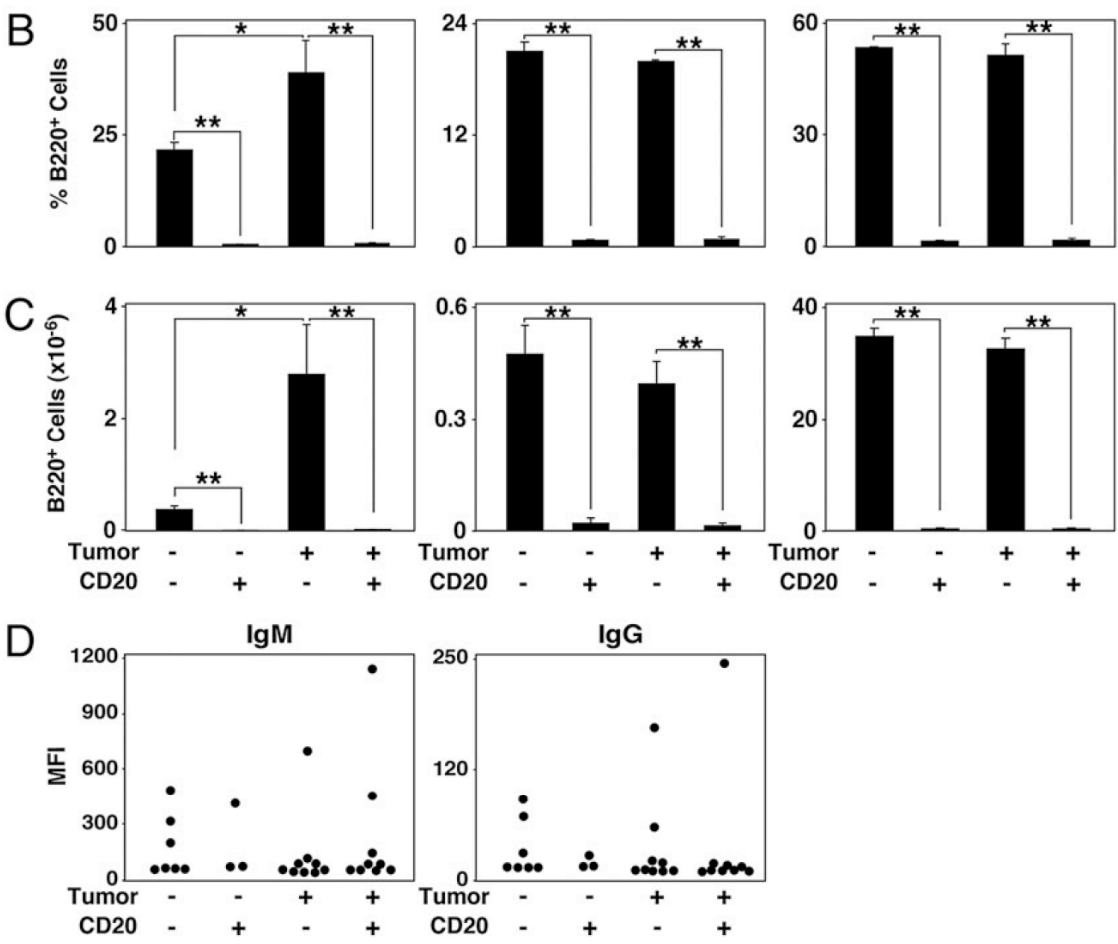

Figure 6: B cell depletion is effective in mice given B16 melanoma. (A) Representative $B$ cell depletion in mice given CD20 or control (CTL) mAb $7 \mathrm{~d}$ before s.c. transfer of 1.5 $x 10^{6}$ B16 / F0 / OVA cells. Fourteen d after tumor transfers, tumor-draining LN (left panels), non-draining LN (middle panels), and spleen (right panels) lymphocytes were isolated and assessed for B220 and CD4 expression by immunofluorescence staining with flow cytometry analysis. B220 $0^{+}$cell percentages within the indicated gates are shown for representative naïve (top panels) or tumor-bearing (bottom panels) mice. (BC) Mean $( \pm$ SEM) B220 $\mathrm{B}$ cell (B) percentages and (C) total numbers in the tissues of control and tumor-bearing mice shown in (A) ( $n=3$ for each group). (D) Ab responses to B16 / F0 / OVA cells are poor. B cells were depleted from mice as in (A), with serum collected from control or CD20 mAb-treated mice $14 \mathrm{~d}$ after tumor transfer. B16/F0 / OVA cells were incubated with diluted serum, washed, and incubated with labeled anti-mouse IgM and IgG Abs before flow cytometry analysis. Circles represent the mean fluorescence intensity (MFI) of stained cells from individual mice that were treated as indicated. (B-D) Significant differences between means are indicated: *, $\mathrm{p}<0.05 ;{ }^{* *}, \mathrm{p}<0.01$. 


\subsubsection{T Cell Numbers are Reduced Following B Cell Depletion}

$\mathrm{T}$ cell and $\mathrm{T}$ cell subset numbers have not been significantly affected by short- ( 7 d) or long-term (28 d) B cell depletion $(27,58)$. To examine this further, spleen and LN T cell numbers were assessed in larger numbers of mice after 21-28 d of control or CD20 $\mathrm{mAb}$ treatment in three independent experiments. On average, there was a $27 \%$ decrease $(\mathrm{p}=0.02)$ in total splenic $\mathrm{CD} 4^{+} \mathrm{T}$ cell numbers in B cell-depleted mice (Figure 7A).

However, there was considerable variability between mice and between experiments. No significant difference was seen for splenic $\mathrm{CD} 8^{+} \mathrm{T}$ cell numbers when the experiments were pooled, although small decreases were observed in two of the three experiments. No differences in T cell numbers were detected within the peripheral LNs of control- and $\mathrm{CD} 20 \mathrm{mAb}$-treated mice (Figure $7 \mathrm{~B})$. In addition, $\mathrm{CD}^{+} \mathrm{CD} 25^{+} \mathrm{FoxP} 3^{+}$regulatory $\mathrm{T}$ cell numbers were modestly decreased in CD20 mAb-treated mice (Table 1). These results suggest a small, but significant decrease in splenic $\mathrm{CD}^{+} \mathrm{T}$ cell numbers over time in naïve mice treated with CD20 mAb. 

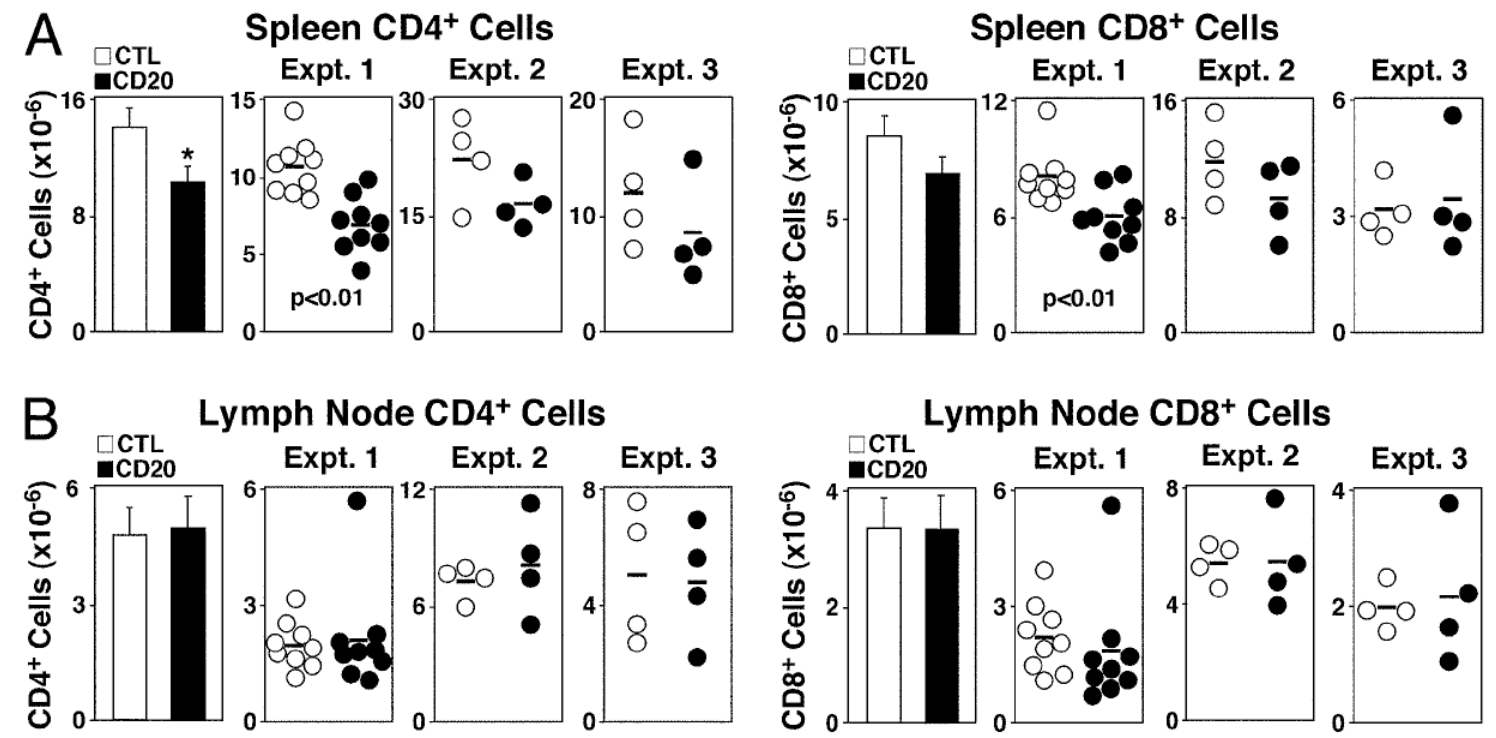

Figure 7: B cell depletion reduces spleen $\mathrm{CD}^{+} \mathrm{T}$ cell numbers. Changes in (A) spleen or (B) peripheral LN CD4 ${ }^{+}$and $\mathrm{CD}^{+} \mathrm{T}$ cell numbers following B cell depletion. Circles represent individual mice that were given control (open) or CD20 (closed) mAb, with spleen and peripheral LN (pooled axial, inguinal, and brachial nodes) lymphocytes harvested 21 (experiment 1) or 28 (experiments 2-3) d later. Bar graphs indicate means $( \pm \mathrm{SEM})$ of pooled $\mathrm{CD} 4^{+}$and $\mathrm{CD} 8^{+} \mathrm{T}$ cell numbers from all three experiments.

Significant differences between means are indicated: ${ }^{*}, \mathrm{p}<0.05$. 
Table 1. $\mathrm{CD}^{+}$and $\mathrm{CD}^{+}$total and naïve $\mathrm{T}$ cell numbers in tumor-bearing mice

\begin{tabular}{|c|c|c|c|c|c|}
\hline \multirow[b]{2}{*}{ Tissue } & \multirow[b]{2}{*}{ Subset } & \multicolumn{2}{|c|}{ Control mice } & \multicolumn{2}{|c|}{ Tumor-bearing mice } \\
\hline & & Control & $\mathrm{CD} 20 \mathrm{mAb}$ & Control & $\mathrm{CD} 20 \mathrm{mAb}$ \\
\hline \multirow[t]{5}{*}{ Spleen: } & Total CD $4^{+}$ & $12.2 \pm 0.6$ & $9.68 \pm 1.06^{*}$ & $11.2 \pm 0.6$ & $8.78 \pm 0.72^{*}$ \\
\hline & Naïve CD4 ${ }^{+}$ & $7.19 \pm 0.43$ & $6.18 \pm 0.83$ & $6.44 \pm 0.47$ & $5.42 \pm 0.56$ \\
\hline & $\begin{array}{c}\mathrm{CD}^{+}{ }^{+} \mathrm{CD} 25^{+} \\
\text {FoxP3 }^{+}\end{array}$ & $1.89 \pm 0.15$ & $1.32 \pm 0.09^{* *}$ & $2.53 \pm 0.24$ & $1.75 \pm 0.18^{*}$ \\
\hline & Total CD8 ${ }^{+}$ & $8.21 \pm 0.41$ & $7.24 \pm 0.62$ & $7.13 \pm 0.49$ & $6.17 \pm 0.58$ \\
\hline & Naïve CD8 ${ }^{+}$ & $5.92 \pm 0.40$ & $5.36 \pm 0.61$ & $4.92 \pm 0.51$ & $4.34 \pm 0.60$ \\
\hline \multirow{5}{*}{$\begin{array}{l}\text { Non-Draining } \\
\text { LN }\end{array}$} & Total CD4 ${ }^{+}$ & $1.94 \pm 0.16$ & $2.02 \pm 0.36$ & $1.42 \pm 0.19$ & $1.44 \pm 0.25$ \\
\hline & Naïve $\mathrm{C}^{+}$ & $1.67 \pm 0.14$ & $1.79 \pm 0.33$ & $1.20 \pm 0.16$ & $1.29 \pm 0.23$ \\
\hline & $\begin{array}{c}\mathrm{CD}^{+}{ }^{+} \mathrm{CD} 25^{+} \\
\text {FoxP3 }^{+}\end{array}$ & $0.67 \pm 0.06$ & $0.36 \pm 0.06^{* *}$ & $0.23 \pm 0.03$ & $0.09 \pm 0.01^{* *}$ \\
\hline & Total CD $8^{+}$ & $1.20 \pm 0.14$ & $1.33 \pm 0.22$ & $1.07 \pm 0.16$ & $0.92 \pm 0.15$ \\
\hline & Naïve CD8 ${ }^{+}$ & $1.27 \pm 0.11$ & $1.14 \pm 0.20$ & $0.87 \pm 0.13$ & $0.78 \pm 0.13$ \\
\hline \multirow[t]{5}{*}{$\begin{array}{c}\text { Tumor- } \\
\text { draining LN }\end{array}$} & Total CD $4^{+}$ & & & $2.47 \pm 0.23$ & $2.29 \pm 0.24$ \\
\hline & Naïve CD $4^{+}$ & & & $2.08 \pm 0.19$ & $2.02 \pm 0.22$ \\
\hline & $\begin{array}{c}\mathrm{CD}^{+} \mathrm{CD}^{2} 5^{+} \\
\text {FoxP3 }^{+}\end{array}$ & & & $0.44 \pm 0.04$ & $0.26 \pm 0.06^{*}$ \\
\hline & Total CD8 ${ }^{+}$ & & & $1.90 \pm 0.18$ & $1.61 \pm 0.18$ \\
\hline & Naïve $C D 8^{+}$ & & & $1.53 \pm 0.14$ & $1.34 \pm 0.15$ \\
\hline
\end{tabular}

Mice were given $\mathrm{CD} 20$ or control $\mathrm{mAb} 7 \mathrm{~d}$ before half of the mice in each group were given 1.5 x 106 s.c. B16/ OVA tumor cells. Tissues were harvested $14 \mathrm{~d}$ later, with total $\mathrm{CD} 4^{+}$and $\mathrm{CD} 8{ }^{+} \mathrm{T}$ cells, naïve $\left(\mathrm{CD} 44^{\mathrm{low}} \mathrm{CD} 62 \mathrm{~L}^{+}\right) \mathrm{CD} 4^{+}$and $\mathrm{CD} 8^{+} \mathrm{T}$ cells, and $\mathrm{CD} 4^{+} \mathrm{CD} 25^{+} \mathrm{FoxP}^{+}$regulatory $\mathrm{T}$ cell numbers $\left(\times 10^{-6}\right)$ determined by immunofluorescence staining with flow cytometry analysis. Values for total and naïve T cells represent 
Table 1 (Continued) means ( \pm SEM) from three groups of three mice in three separate experiments. Values for $\mathrm{CD} 4{ }^{+} \mathrm{CD} 25^{+} \mathrm{FoxP} 3^{+}$regulatory $\mathrm{T}$ cells represent means $( \pm \mathrm{SEM})$ from five mice in each group. Non-draining LNs in control mice consist of pooled bilateral inguinal, brachial, and axial LNs. Significant differences between sample means are indicated: ${ }^{*}, \mathrm{p} \leq 0.05$. 


\subsubsection{B Cell Depletion Impairs CD4 ${ }^{+}$and $\mathrm{CD}^{+}$Effector-Memory T Cell Induction}

Whether naïve or effector-memory T cell subset numbers decreased in B cell-depleted mice was assessed in mice given B16 tumors. Mice were given control or CD20 mAb 7 d before the s.c. transfer of B16 / F0 / OVA cells into half of the mice, with CD44 and CD62L expression by $\mathrm{CD}^{+}$or $\mathrm{CD}^{+} \mathrm{T}$ cells assessed $14 \mathrm{~d}$ after tumor transfers.

Overall, B cell depletion did not have a significant effect on naïve (CD44 $\left.{ }^{\text {lo }} \mathrm{CD} 62 \mathrm{~L}^{\mathrm{hi}}\right)$ $\mathrm{CD}^{+}$or $\mathrm{CD}^{+} \mathrm{T}$ cell numbers in control or tumor-bearing mice (Table I). However, B cell depletion in control mice significantly reduced spleen $\mathrm{CD} 4^{+}$effector-memory $\left(\mathrm{CD} 44^{\mathrm{hi}} \mathrm{CD} 62 \mathrm{~L}^{\mathrm{lo}}\right) \mathrm{T}$ cell numbers (39\% decrease, $\mathrm{p}=0.0005$; Figure $\left.8 \mathrm{~A}\right)$. While effectormemory $\mathrm{T}$ cell numbers were increased $>3$-fold within the draining LNs of control $\mathrm{mAb}$ treated mice with B16/F0/OVA tumors $(\mathrm{p}<0.001)$, this increase was significantly reduced in B cell-depleted mice $(42-48 \%$ decrease, $\mathrm{p} \leq 0.002)$ when compared with control mAb-treated mice with tumors. Similar differences were observed in non-tumor draining LNs, with significantly fewer (49\% decrease, $\mathrm{p}=0.01) \mathrm{CD} 4^{+}$effector-memory cells in B cell-depleted mice when compared with control mAb-treated mice. CD4 ${ }^{+}$ effector-memory $\mathrm{T}$ cell percentages and numbers were also decreased within the spleens of tumor-bearing mice treated with CD20 versus control mAb (21-38\% decreases, $\mathrm{p} \leq 0.04)$. Similar results were observed in mice given s.c. B16/F10 cells or i.v. B16 / F0 / OVA cells (data not shown). $\mathrm{CD}^{+}$effector-memory $\mathrm{T}$ cell numbers were also decreased in B cell-depleted mice that received B16 / F0 / OVA cells at low doses (Figure 8C-D).

There were no significant differences in $\mathrm{LN} \mathrm{CD} 8^{+}$effector-memory $\mathrm{T}$ cell percentages or numbers between control and CD20 mAb-treated mice (Figure 8B). However, B cell depletion did reduce $\mathrm{CD}^{+}$effector-memory $\mathrm{T}$ cell induction in mice with tumors. There were significantly fewer ( $33 \%$ decrease, $\mathrm{p}=0.01) \mathrm{CD} 8^{+}$effector-memory $\mathrm{T}$ cells within 
the draining LNs of B cell-depleted mice with tumors when compared to control mAbtreated mice with tumors. A similar trend was observed in the non-tumor draining LNs. Spleen $\mathrm{CD}^{+}$effector-memory $\mathrm{T}$ cell numbers were also decreased by $29-31 \%(\mathrm{p}=0.03)$ in B cell-depleted mice when compared with control mAb-treated mice, and in tumorbearing mice given CD20 mAb in comparison with control mAb treatment. Decreased $\mathrm{CD}^{+}$effector-memory cell numbers were also seen in B cell-depleted mice that received low doses of B16/F0 / OVA cells (Figure 8E-F). Similar results were observed in mice given s.c. B16 / F10 cells or i.v. B16 / F0 / OVA cells (data not shown). B cell depletion did not induce the apoptosis of effector-memory $\mathrm{CD} 4^{+}$and $\mathrm{CD} 8^{+}$cells, because Annexin- $\mathrm{V}$ staining of these populations was comparable between tumor-bearing control and CD20 mAb-treated mice (data not shown). Collectively, these results demonstrate that B cell depletion significantly impairs effector-memory $\mathrm{CD} 4^{+}$and $\mathrm{CD} 8^{+} \mathrm{T}$ cell induction. 

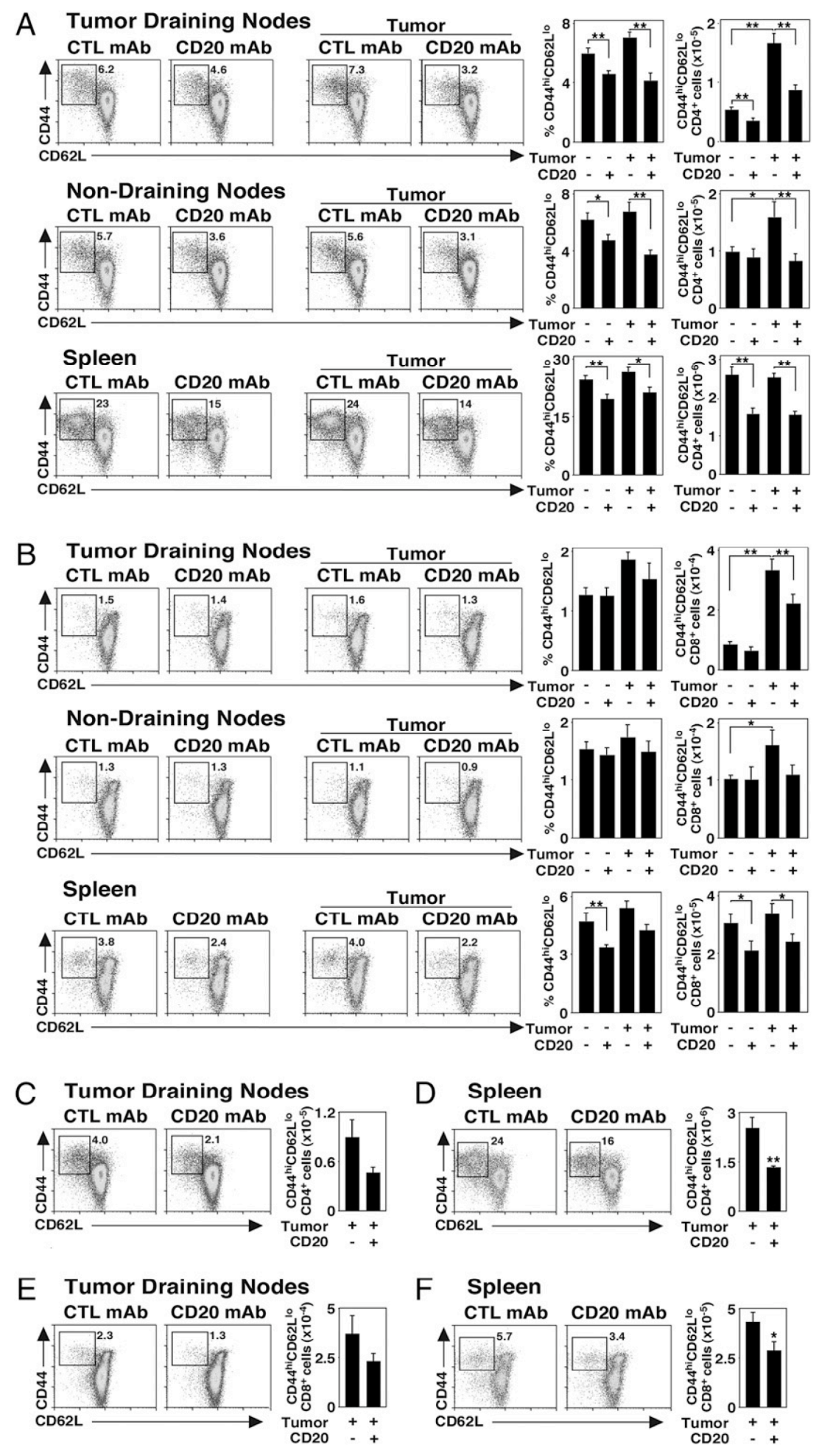

Figure 8: Effector-memory $T$ cell induction is impaired in B cell-depleted tumorbearing mice. Mice were given CD20 or control (CTL) $\mathrm{mAb} 7 \mathrm{~d}$ before half the mice in 
Figure 8 (Continued) each group received $1.5 \times 10^{6}$ s.c. B16/F0 / OVA cells. Fourteen d later, the frequencies and numbers of $(\mathrm{A}) \mathrm{CD} 44{ }^{\mathrm{hi}} \mathrm{CD} 62 \mathrm{~L}^{\mathrm{lo}} \mathrm{CD} 4{ }^{+}$and (B) CD $44^{\mathrm{hi}} \mathrm{CD} 62 \mathrm{~L}^{\mathrm{lo}}$ $\mathrm{CD}^{+} \mathrm{T}$ cells within draining LNs, non-draining LNs, and spleens were determined by immunofluorescence staining with flow cytometry analysis $(\mathrm{n}=9$ for each group, pooled from three separate experiments in which $n=3)$. C-F) Mice, treated as in (A-B), received $1 \times 10^{5} \mathrm{~B} 16 / \mathrm{F} 0 /$ OVA cells, with $(\mathrm{C}-\mathrm{D}) \mathrm{CD} 44^{\text {hi }} \mathrm{CD} 62 \mathrm{~L}^{\mathrm{lo}} \mathrm{CD} 4{ }^{+}$and (E-F)

$\mathrm{CD} 44^{\mathrm{hi}} \mathrm{CD} 62 \mathrm{~L}^{\text {lo }} \mathrm{CD} 8^{+} \mathrm{T}$ cell frequencies and numbers analyzed for tumor draining $\mathrm{LNs}$ and the spleen ( $n=5$ per group). Representative dot plots gated on CD44 hi CD62L ${ }^{\text {ho }} \mathrm{T}$ cells are shown, with relative percentages indicated. Bar graphs indicate the mean $( \pm$ SEM) percentages and numbers of $\mathrm{CD} 44{ }^{\mathrm{hi}} \mathrm{CD} 62 \mathrm{~L}^{\text {lo }}$ cells in the indicated treatment groups. Significant differences between sample means are indicated: ${ }^{*}, \mathrm{p}<0.05$; ${ }^{* *}$, $\mathrm{p}<0.01$. 


\subsubsection{B Cell Depletion Impairs IFN $\gamma$ and TNF $\alpha$ Induction by $\mathrm{CD}^{+}$and CD8 $^{+}$T Cells In Vivo}

The in vivo effects of B cell depletion on IFN $\gamma$ and TNF $\alpha$ production by CD4 ${ }^{+}$ and $\mathrm{CD}^{+} \mathrm{T}$ cells were quantified as important markers for $\mathrm{T}$ cell stimulation due to their roles in tumor immunity. Mice were given control or CD20 mAb $7 \mathrm{~d}$ before B16 / F0 / OVA cells were transferred s.c. into half of the mice. Tumor draining LN, contralateral LN, and spleen lymphocytes were isolated $14 \mathrm{~d}$ after tumor transfers, with $\mathrm{B}$ cells removed using magnetic beads. The remaining non-B cells were cultured in vitro with plate-bound CD3 and CD28 mAbs for $3.5 \mathrm{~h}$ to identify T cells that were actively producing cytokines in vivo. The cells were stained for cell surface CD4 and CD8 as well as cytoplasmic IFN $\gamma$ and TNF $\alpha$ expression, and were analyzed by flow cytometry. Comparisons were made between control and CD20 mAb-treated mice bearing tumors that were $<0.25 \mathrm{~cm}^{3}$. B cell-depleted mice bearing tumors $\geq 0.25 \mathrm{~cm}^{3}$ were also analyzed, but their cytokine-secreting $\mathrm{T}$ cell numbers increased significantly with tumor size.

Percentages and numbers of IFN $\gamma$ - and TNF $\alpha$-secreting $\mathrm{CD}^{+}{ }^{+} \mathrm{T}$ cells were similar in the LNs of control mAb- or CD20 mAb-treated mice not given tumors (Figure 9A-B). By contrast, total $\mathrm{IFN} \gamma^{+}$and $\mathrm{TNF} \alpha^{+} \mathrm{CD}^{+} \mathrm{T}$ cell numbers within tumor-draining LNs were increased in control mAb-treated mice $(\sim 3.5$-fold, $\mathrm{p}<0.001)$ when compared to control mAb-treated mice not given tumors. The draining LNs of B cell-depleted mice with $<0.25 \mathrm{~cm}^{3}$ tumors had significantly smaller percentages $(42-45 \%$ decrease, $\mathrm{p} \leq 0.04)$ and numbers (42-47\% decrease, $\mathrm{p} \leq 0.02)$ of IFN $\gamma^{+}$and $\mathrm{TNF} \alpha^{+} \mathrm{CD}^{+} \mathrm{T}$ cells (Figure 9A). Similar results were observed for IFN $\gamma^{+} \mathrm{CD}^{+} \mathrm{T}$ cells in the non-draining LNs (Figure 9B). By contrast, B cell depletion did not significantly affect the percentages or numbers of IFN $\gamma^{+}$and $\mathrm{TNF}^{+} \mathrm{CD}^{+}$cells within tumor draining $\mathrm{LNs}$ of mice with $\geq 0.25 \mathrm{~cm}^{3}$ tumors. There was a $44 \%$ decrease $(\mathrm{p} \leq 0.01)$ in $\mathrm{IFN}^{+} \mathrm{CD}^{+} \mathrm{T}$ cell numbers and a $34 \%$ decrease $(\mathrm{p}=0.03)$ in $\mathrm{TNF} \alpha^{+} \mathrm{CD} 4^{+} \mathrm{T}$ cell numbers in the spleens of $\mathrm{B}$ cell-depleted mice not given 
tumors compared to control mAb-treated littermates (Figure 9C). The spleens of B celldepleted mice with tumors also contained significantly fewer (39\% decrease, $\mathrm{p}=0.02$ ) IFN $\gamma^{+} \mathrm{CD} 4^{+} \mathrm{T}$ cells when compared with control mAb-treated littermates with tumors. Thus, B cell depletion impaired the induction of IFN $\gamma$ - and $\mathrm{TNF} \alpha$-secreting $\mathrm{CD}^{+} \mathrm{T}$ cells in response to $\mathrm{B} 16 / \mathrm{F} 0 / \mathrm{OVA}$ cells.

B cell depletion also affected the generation of cytokine-secreting $\mathrm{CD} 8^{+}$cells (Figure 10). CD20 mAb treatment did not affect $\mathrm{IFN} \gamma^{+}$or $\mathrm{TNF} \alpha^{+} \mathrm{CD} 8^{+} \mathrm{T}$ cell frequencies or numbers in mice not given tumors, except for $\mathrm{TNF} \alpha$-secreting $\mathrm{CD} 8^{+} \mathrm{T}$ cells within the spleen. IFN $\gamma^{+}$and $\mathrm{TNF} \alpha^{+} \mathrm{CD}^{+} \mathrm{T}$ cell frequencies and numbers increased $\geq 3.2$-fold $(\mathrm{p}=0.0007)$ within LNs in control mAb-treated mice with tumors compared to control mAb-treated mice not given tumors (Figure 10A). However, B cell depletion decreased the percentages ( $21 \%$ decrease, $\mathrm{p}=0.02)$ and numbers ( $34 \%$ decrease) of IFN $\gamma^{+} \mathrm{CD} 8^{+} \mathrm{T}$ cells within tumor-draining nodes of mice when compared to control mAb-treated mice with tumors. B cell depletion also reduced $\mathrm{TNF}^{+} \mathrm{CD}^{+} \mathrm{T}$ cell numbers in the draining LNs of mice bearing $<0.25 \mathrm{~cm}^{3}$ tumors by $50 \%$ ( $\left.\mathrm{p}=0.007\right)$ when compared to control mAb-treated mice with tumors. Similar frequencies and numbers of IFN $\gamma^{+}$and $\mathrm{TNF}^{+}$ $\mathrm{CD}^{+} \mathrm{T}$ cells were found within non-tumor draining nodes of CD20 and control mAbtreated mice (Figure 10B). Few differences were seen in splenic IFN $\gamma^{+}$and $\mathrm{TNF}^{+} \mathrm{CD}^{+} \mathrm{T}$ cell numbers between the experimental groups (Figure 10C). Thus, B cell depletion predominantly reduced IFN $\gamma$ or $\mathrm{TNF} \alpha$ expression by $\mathrm{CD}^{+} \mathrm{T}$ cells within tumor-draining LNs. 
A Tumor Draining Nodes
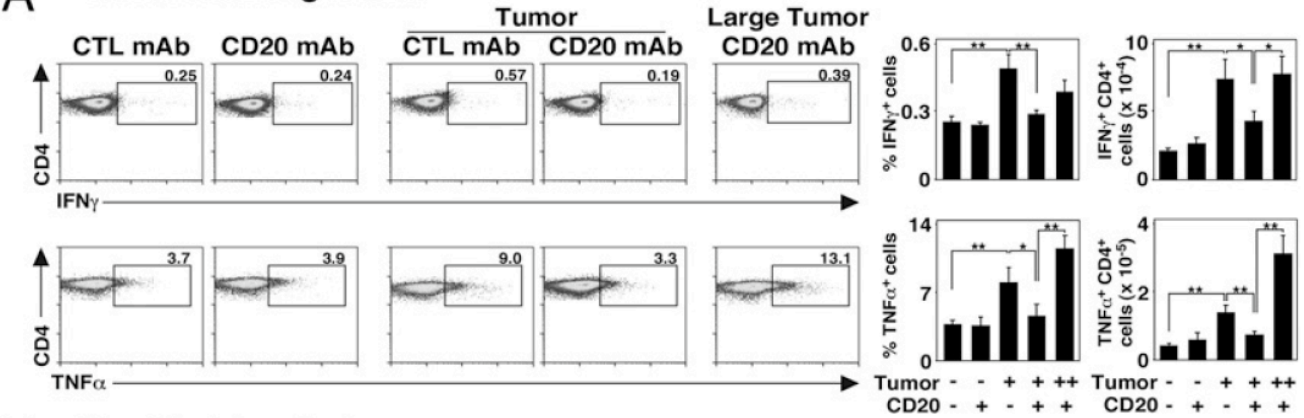

\section{B Non-Draining Nodes}
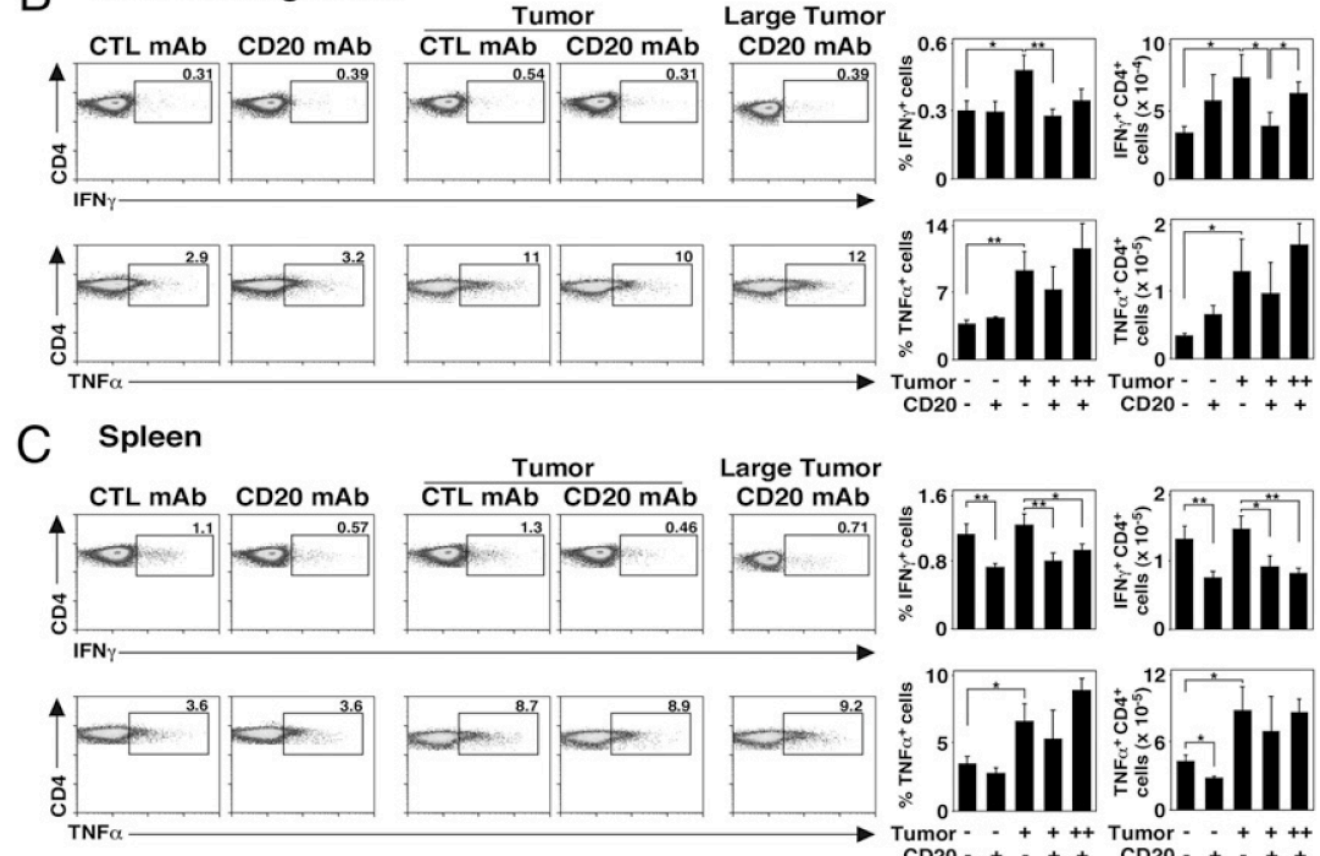

Tumor - - + ++ Tumor - - + + +
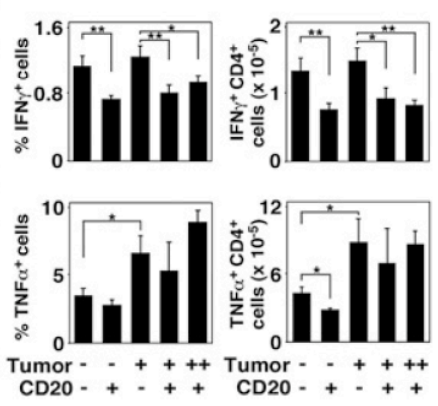

Figure 9: B cell depletion impairs the induction of IFN $\gamma$ - and TNF $\alpha$-secreting CD4 ${ }^{+}$ cells in tumor-bearing mice. Mice were given CD20 or control (CTL) mAb $7 \mathrm{~d}$ before half the mice in each group received $1.5 \times 10^{6}$ s.c. B16/F0 / OVA cells. Fourteen d later, draining and non-draining LNs and spleens were isolated, and B cells were removed using CD19 mAb-coated magnetic beads. The remaining non-B cells were cultured for $3.5 \mathrm{~h}$ with plate-bound CD3/CD28 mAbs before cell surface CD4 labeling and intracellular cytokine staining with flow cytometry analysis. Representative dot plots are shown for (A) tumor draining LNs, (B) non-tumor draining LNs, and (C) spleen CD4 ${ }^{+} \mathrm{T}$ cell expression of cytoplasmic IFN $\gamma$ (upper panels) or TNF $\alpha$ (lower panels). Bar graphs indicate mean ( \pm SEM) frequencies (left histograms) and numbers (right histograms) of $\mathrm{CD} 4{ }^{+} \mathrm{IFN} \gamma^{+}$(upper panels) and $\mathrm{CD}^{+}{ }^{+} \mathrm{TNF} \alpha^{+}$(lower panels) cells in the indicated tissues of mice ( $n=6$ for tumor-free control mice, $n=6$ for tumor-free CD20 mAb-treated mice, $\mathrm{n}=11$ for tumor-treated control mice, and $\mathrm{n}=5$ for CD2 $0 \mathrm{mAb}$-treated mice with $<0.25$ $\mathrm{cm}^{3}$ tumors). Results from mice with tumors $\geq 0.25 \mathrm{~cm}^{3}(++)$ are also indicated $(\mathrm{n}=7)$. Significant differences between sample means are indicated: ${ }^{*}, \mathrm{p}<0.05 ;{ }^{* *}, \mathrm{p}<0.01$. 

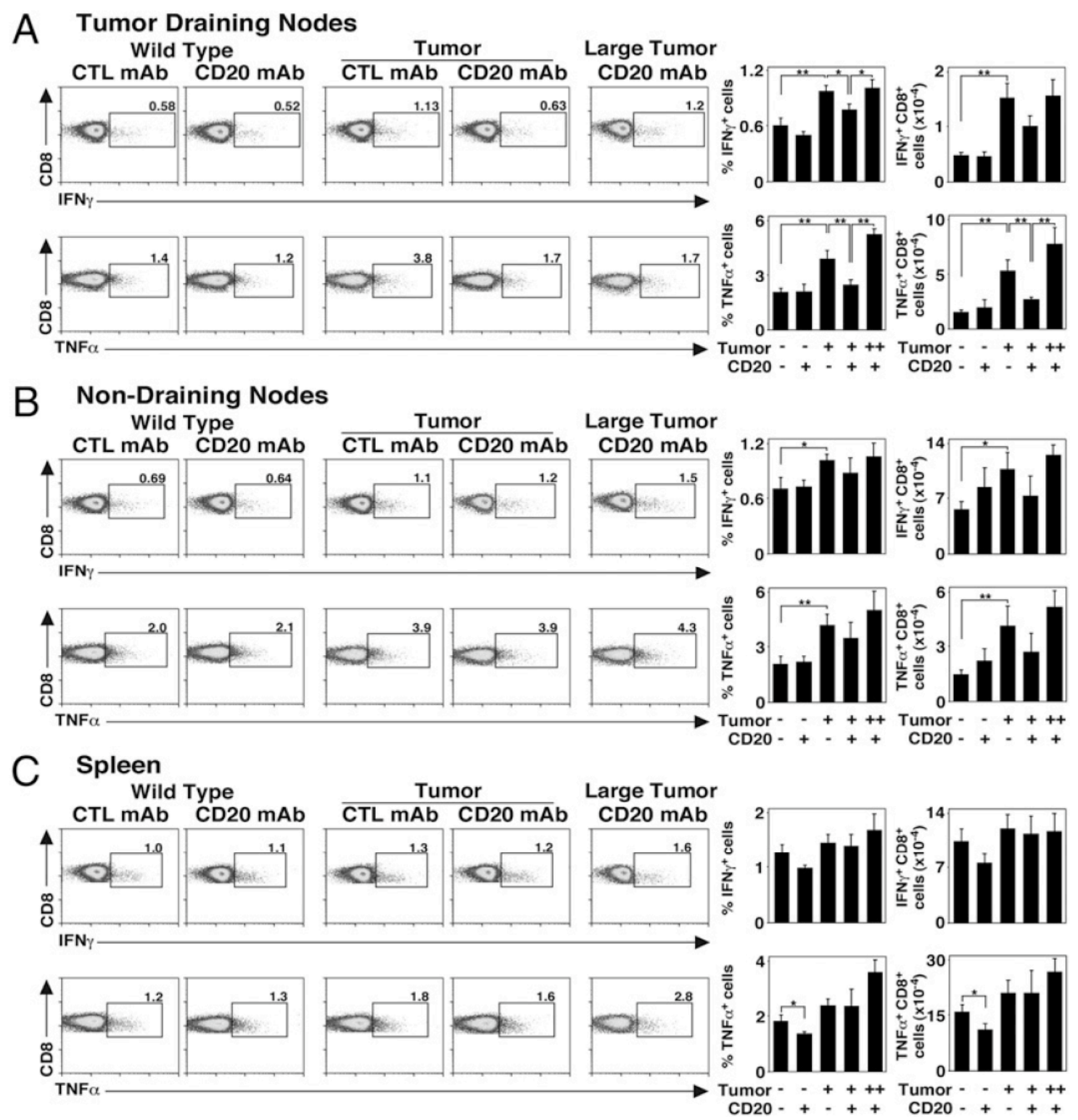

Figure 10: B cell depletion impairs the induction of IFN $\gamma$ - and TNF $\alpha$-secreting CD8 ${ }^{+}$ cells in tumor-bearing mice. Mice were treated as outlined in Figure 9, except T cells expressing cell surface CD8 from (A) tumor draining LNs, (B) non-draining LNs, and (C) spleen were analyzed for cytoplasmic IFN $\gamma$ (upper panels) or TNF $\alpha$ (lower panels) expression. Data presented are as outlined in Figure 9. 


\subsubsection{B Cells Are Required for Optimal T Cell Cytokine Induction In Vitro}

To further verify a role for B cells in T cell cytokine induction in vivo, similar studies were carried out in vitro using purified B cells in short-term $\mathrm{T}$ cell induction assays. Splenic CD19+ $\mathrm{B}$ cells $\left(>99 \% \mathrm{CD}^{+}{ }^{+}\right)$and $\mathrm{CD}^{-} 9^{-}$cells ( $\mathrm{T}$ cells, $<2 \% \mathrm{CD}^{-} 9^{+}$cells) were purified and cultured with plate-bound CD3 / CD28 mAbs for $3.5 \mathrm{~h}$ at varying T:B cell ratios before cell surface CD4 and CD8 staining, intracellular IFN $\gamma$ and TNF $\alpha$ staining, and flow cytometry analysis. Plate-bound T cell stimulation was used to reduce the signaling contributions from B cell Fc receptor-mediated crosslinking of the CD3/CD28 mAbs that occurs with soluble mAbs. As B cells were increasingly added to the $\mathrm{T}$ cell cultures, there were significant increases in the frequencies of $\mathrm{T}$ cells expressing detectable intracellular IFN $\gamma$ and TNF $\alpha$. There was a 2-fold increase $(p=0.0003)$ in CD4 $4^{+}$ T cells producing IFN $\gamma$ when B cells were present at 1:9 T:B cell ratios in comparison with $\mathrm{T}$ cells cultured alone (Figure 11A). Similarly, TNF $\alpha$-producing $\mathrm{CD}^{+} \mathrm{T}$ cell numbers increased 2.4-fold ( $\mathrm{p}=0.001)$ when B cells were present at 1:9 T:B cell ratios in comparison with T cells alone (Figure 11B). Significantly enhanced frequencies of CD8 ${ }^{+}$ T cells produced IFN $\gamma$ (3-fold increase, $\mathrm{p}=0.0005)$ and TNF $\alpha$ (4.4-fold increase, $\mathrm{p}<0.0001$ ) when B cells were present in the cultures (Figure 11C-D). By contrast, adding B cells to the cultures did not enhance the ability of unstimulated $\mathrm{T}$ cells to produce cytokines. Thereby, the presence of B cells significantly enhanced $\mathrm{CD} 4^{+}$and $\mathrm{CD} 8^{+} \mathrm{T}$ cell cytokine production in response to CD3 / CD28 stimulation. 

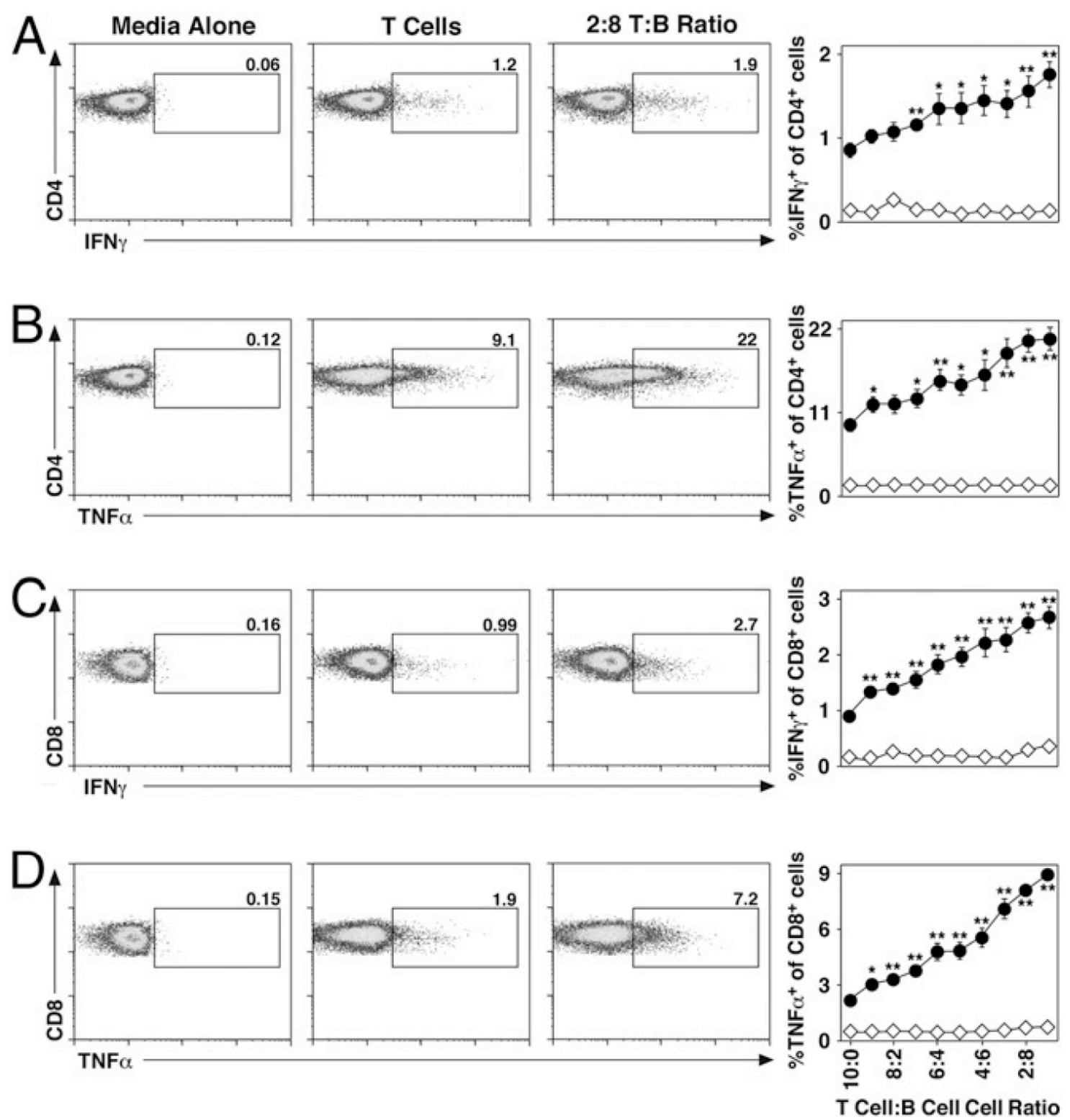

Figure 11: B cells are required for optimal $\mathrm{T}$ cell cytokine induction in vitro. Purified splenic B cells (>99\% CD19 ${ }^{+}$) and total splenocytes depleted of CD19 ${ }^{+}$cells (T cells, $<2 \% \mathrm{CD} 19^{+}$) from naïve wild type mice were cultured for $3.5 \mathrm{~h}$ in media alone (open diamonds) or with plate-bound CD3/CD28 mAbs (filled circles) at varying T:B cell ratios $\left(10^{6}\right.$ total lymphocytes per well) before staining for cell surface CD4 or CD8 and cytoplasmic cytokine expression with flow cytometry analysis. Representative flow cytometry analysis (left panels) of $\mathrm{CD}^{+} \mathrm{T}$ cells expressing cytoplasmic (A) IFN $\gamma$ or (B) $\mathrm{TNF} \alpha$, or $\mathrm{CD}^{+} \mathrm{T}$ cells expressing (C) IFN $\gamma$ or (D) TNF $\alpha$ are shown for various culture conditions. Line graphs indicate mean $( \pm$ SEM) results from triplicate culture wells, and are representative of the results obtained in two independent experiments. Significant differences between sample means are indicated: ${ }^{*}, \mathrm{p}<0.05 ;{ }^{* *}, \mathrm{p}<0.01$. 


\subsubsection{B cell Depletion Impairs Tumor Ag-Specific $\mathrm{CD}^{+}{ }^{+}$Cell Proliferation In Vivo}

The role of B cells in tumor Ag-specific $\mathrm{CD}^{+}$and $\mathrm{CD} 8^{+} \mathrm{T}$ cell proliferation in vivo was assessed directly using the B16/F0 / OVA cell line that secretes OVA protein, and OVA peptide-specific Thy $1.1^{+} \mathrm{CD}^{+}$and $\mathrm{CD}^{+} \mathrm{T}$ cells from OT-II and OT-I transgenic mice, respectively $(100,101)$. In agreement with previous studies $(136,137)$, OT-II cells did not proliferate in vivo in response to B16 / F0 / OVA tumors, even when harvested $10 \mathrm{~d}$ after transfer into tumor-bearing mice, while OT-I T cells proliferated in response to B16/F0/OVA tumors (data not shown). Mice were therefore treated with control or CD20 mAb 6 d before receiving B16/F0 / OVA cells i.v. The following day, CFSE-labeled CD8 ${ }^{+}$OT-I T cells were adoptively transferred into the mice, with lungdraining hilar LNs, peripheral LNs, and spleens harvested 5 or 9 d later. Identical results were observed at both time points, so the data were pooled. There were no differences in OT-I T cell proliferation or numbers in hilar LNs (Figure 12A). However, OT-I T cell proliferation was significantly reduced within peripheral LNs ( $27 \%$ decrease, $\mathrm{p}=0.003)$ and spleens $(35 \%$ decrease, $\mathrm{p}<0.0001)$ of $\mathrm{B}$ cell-depleted mice when compared with control mAb-treated littermates. Thus, B cell depletion did not reduce $\mathrm{T}$ cell entry into peripheral LNs, but did reduce transgenic T cell proliferation. The significantly greater numbers of OVA-secreting tumor cells within the lungs of B cell-deficient mice compared to control mAb-treated mice (Figure 5) may explain why OT-I cell proliferation was not reduced by B cell depletion within the lung-draining hilar LNs. Nonetheless, these results directly demonstrate that $\mathrm{CD}^{+} \mathrm{T}$ cell expansion in response to tumor-specific Ags is impaired in B cell-depleted hosts. 


\subsubsection{B Cell Depletion Does Not Affect the Growth of Established Tumors}

Whether B cell depletion enhances the growth of established tumors was assessed in mice given s.c. injections of $1 \times 10^{5}$ wild type B16/F10 cells $7 \mathrm{~d}$ before treatment with either control or CD20 mAb. Tumors in both control and CD20 mAb-treated mice grew with similar kinetics, and no significant differences were seen in tumor volumes $14 \mathrm{~d}$ after tumor challenge (Figure 12B). Thus, established tumors grow equally well in the presence and absence of B cells. 

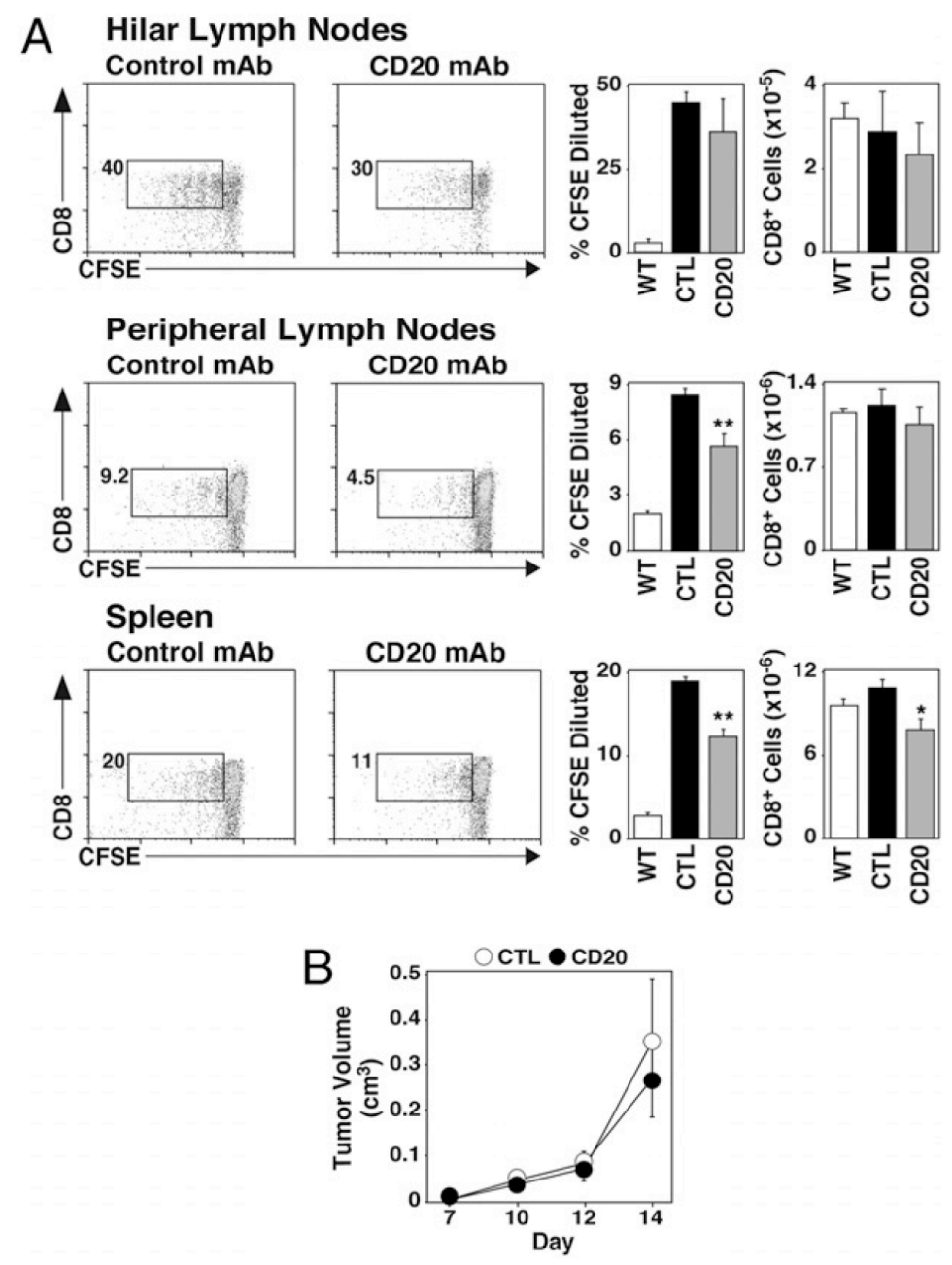

Figure 12: B cell depletion impairs tumor Ag-specific $\mathrm{CD8}^{+} \mathrm{T}$ cell proliferation, but does not affect the growth of pre-established tumors. (A) Mice were given CD20 or control (CTL) mAb $6 \mathrm{~d}$ before receiving B16/F0 / OVA cells i.v. One $\mathrm{d}$ later, the mice were adoptively-transferred with CFSE-labeled enriched Thy $1.1^{+} \mathrm{CD} 8^{+} \mathrm{T}$ cells from OTI mice. Lymphocytes from lung-draining hilar LNs, peripheral LNs, and spleens were isolated 5 or $9 \mathrm{~d}$ after OT-I cell transfer in two separate experiments, with CD8 expression and CFSE dilution for Thy $1.1^{+} \mathrm{T}$ cells assessed by flow cytometry analysis. The results from the 5 and $9 \mathrm{~d}$ studies were similar, if not identical, so the data were pooled. Representative CFSE versus cell surface CD8 staining for Thy $1.1^{+}$cells is shown, with the percentages of CFSE-diluted $\mathrm{CD}^{+}$cells within each gate indicated as a fraction of total CD $8^{+}$Thy $1.1^{+} \mathrm{T}$ cells. Bar graphs show the mean percentage $( \pm$ SEM) of CFSEdiluted cells from tumor-free wild type mice (open bars, $n=3$ ), tumor-bearing control mAb-treated mice (closed bars, $n=6$ ), and tumor-bearing CD20 mAb-treated mice (shaded bars, $n=6$ ). The far right bar graphs show total numbers of $C D 8^{+} \mathrm{T}$ cells within each tissue. Significant differences between sample means are indicated: ${ }^{*}, \mathrm{p}<0.05$; ${ }^{* *}$, $\mathrm{p}<0.01$. (B) Mice were given CD20 or control mAb $7 \mathrm{~d}$ after s.c. transfer of $10^{5} \mathrm{~B} 16 / \mathrm{F} 10$ cells, with tumor volumes measured on the indicated days. 


\subsection{Discussion}

Mature B cell depletion in adult mice dramatically enhanced B16 melanoma tumor growth. Subcutaneous melanoma tumors were $\sim 2$-fold larger and observable melanoma metastasis was enhanced $\sim 2$-fold in the absence of B cells (Figure 5). B cell depletion had modest, if any, effects on naïve T cell numbers (Table 1). However, the induction of $\mathrm{CD}^{+}$and $\mathrm{CD}^{+}$effector-memory T cells (Figure 8 ) and inflammatory and cytotoxic cytokine-secreting T cells (Figure 9, Figure 10) was significantly impaired in B celldepleted mice. Consistent with this, the presence of B cells significantly enhanced the frequency of cytokine-secreting $\mathrm{T}$ cells during short-term $(3.5 \mathrm{~h})$ in vitro cultures (Figure 11). The ability of adoptively-transferred OVA-specific $C D 8^{+} \mathrm{T}$ cells to proliferate in response to B16/F0/OVA tumors in vivo was also impaired in the absence of B cells (Figure 12). Thus, these studies collectively demonstrate that B cells are required for optimal $\mathrm{T}$ cell activation and the induction of cellular immunity in this in vivo nonlymphoid tumor model. Thereby, while B cells may not have direct effector roles in tumor immunity, impaired $\mathrm{T}$ cell activation and effector-memory cell generation in the absence of B cells is likely to promote tumor growth in these immunocompromised hosts.

B cells can present Ags more efficiently than other APCs, and can present tumor Ags and autoAgs efficiently when the Ags are present at low concentrations $(27,51,138)$. Specifically, B cells are not required for optimal $\mathrm{CD} 4^{+} \mathrm{T}$ cell activation at high $\mathrm{Ag}$ concentrations, while this responsibility is shared by B cells and DCs when Ag or autoAg concentrations are low (27). Furthermore, B cell MHC class II expression is required for maximal Ag-specific $\mathrm{CD} 4^{+} \mathrm{T}$ cell expansion, $\mathrm{CD} 4^{+}$memory formation, and $\mathrm{CD}^{+} \mathrm{T}$ cell cytokine production in vivo $(19,20)$. In addition, proteogylcan-induced arthritis is ameliorated and $\mathrm{T}$ cell activation and cytokine production are impaired in mice depleted of B cells with CD20 mAb $(43,139)$. B cells can also provide potent 
costimulatory signals for T cell expansion (30). In the case of weakly immunogenic tumors like B16 melanoma, the current studies demonstrate that B cells and other APCs are both involved in optimal $\mathrm{CD} 4^{+} \mathrm{T}$ cell expansion (Figs. 4-8). B cells have also been implicated in the contraction of $\mathrm{CD}^{+} \mathrm{T}$ cell responses and formation of memory $\mathrm{CD} 8^{+} \mathrm{T}$ cells during infections (140). Defective $\mathrm{CD}^{+} \mathrm{T}$ cell activation and memory formation may also result from impaired $\mathrm{CD}^{+} \mathrm{T}$ cell activation in the absence of B cells. In fact, the transfer of CD40-activated, tumor peptide-loaded B cells induces Ag-specific antitumor immune responses in mice via cross-presentation of Ag to APCs as well as direct T cell activation by the B cells (141). It is also possible that B cells may be responsible for generating Abs against disseminated forms of tumor Ags or their shed products (i.e. exosomes, gangliosides, B7-H1, etc.) that, at least locoregionally, promote the premature death of tumor-Ag experienced, but not naïve, $\mathrm{T}$ cells. Taken together, the results indicate that the absence of $\mathrm{B}$ cells during both the activation and expansion of tumor Ag-specific T cells explains the significant effects on both $\mathrm{CD} 4^{+}$and $\mathrm{CD} 8^{+} \mathrm{T}$ cellmediated tumor immunity observed in this study.

We have previously reported that $\mathrm{CD} 4^{+}$and $\mathrm{CD} 8^{+} \mathrm{T}$ cell numbers do not change significantly after in vivo B cell depletion using CD20 mAbs $(27,58)$. While the current studies demonstrate that naïve $\mathrm{CD}^{+}$and $\mathrm{CD} 8^{+} \mathrm{T}$ cell numbers predominantly remain constant within tissues after B cell depletion for $21 \mathrm{~d}$, there was a significant reduction in $\mathrm{CD} 44^{\text {hi }} \mathrm{CD}^{2} 2 \mathrm{~L}^{\text {lo }}$ effector-memory $\mathrm{T}$ cells that affected total $\mathrm{CD} 4{ }^{+}$and $\mathrm{CD} 8^{+} \mathrm{T}$ cell numbers (Table 1, Figure 7, Figure 8). Thus, even though T cell numbers varied between mice after CD20 mAb treatment and between experiments (Figure 7), there was a 2027\% decrease in spleen T cell numbers following prolonged B cell depletion (Figure 7, Table 1). It is unlikely that B cell depletion induces the death of $\mathrm{T}$ cells, since there is no decrease in total or effector-memory $\mathrm{CD}^{+}$or $\mathrm{CD}^{+} \mathrm{T}$ cell numbers $7 \mathrm{~d}$ after CD20 mAb injection (27), and effector-memory $\mathrm{CD} 4^{+}$or $\mathrm{CD} 8^{+} \mathrm{T}$ cells from the draining LNs and 
spleen show similar Annexin-V staining in both control and CD20 mAb-treated mice (data not shown). Thus, the decrease in effector-memory T cell numbers 21 and $28 \mathrm{~d}$ after B cell depletion is most likely due to the impaired repopulation of effector-memory cells during normal turnover due to the suboptimal activation of $\mathrm{T}$ cells in the absence of B cells. That $\mathrm{T}$ cell numbers were not found to be significantly altered in previous experiments is likely due to short CD20 mAb treatment times, variability in effectormemory $\mathrm{T}$ cell subsets between groups of mice, insufficient numbers of mice within groups to detect small differences, or cell numbers were enumerated after immunizations with Ags in adjuvant. Modest decreases in $\mathrm{CD} 4^{+}$and $\mathrm{CD} 8^{+}$memory $\mathrm{T}$ cell frequencies have also been noted in human CD20 transgenic MRL/lpr mice 10 weeks after treatment with $20 \mathrm{mg} /$ week of an anti-human CD20 mAb (142). Nonetheless, the small decrease in $\mathrm{T}$ cell numbers observed in CD20 mAb-treated mice is consistent with decreased $\mathrm{CD}^{+} \mathrm{T}$ cell numbers in the spleens of B cell-deficient $\mu \mathrm{MT}$ mice (143).

Previous studies using non-lymphoid tumor lines in $\mu \mathrm{MT}$ mice have found that antitumor Th1 and cytotoxic T cell responses are enhanced and tumor growth is generally reduced in the absence of B cells $(83-85,144)$. Furthermore, reconstitution of $\mu$ MT mice with wild type B cells abrogates their tumor resistance (84). In the absence of B cells, enhanced in vitro anti-tumor responses have also been observed following tumor Ag vaccinations $(85,144)$. That the $\mathrm{T}$ cell repertoire, lymphoid tissue architecture, and macrophage and DC numbers and function are all established in a wild type environment prior to B cell depletion using CD20 mAbs are the most likely explanations for differences between the current studies and previous studies using $\mu \mathrm{MT}$ mice $(27,39$, $68,71)$. In addition, the pro- or anti-tumor impact of B cells is likely to depend heavily on the tumor model being examined, due to the complex roles that B cells contribute to tumor immunity and other immune responses $(116,128)$. Regardless, the current studies 
using B cell-depleted mice collectively demonstrate that B cells unequivocally contribute to the development of anti-tumor immunity. In previous studies, an ability of B cells to negatively regulate anti-tumor immune responses was attributed to B cell provision of IL-10 (83), most likely by regulatory B10 cells $(68,116)$. In addition, partial B cell depletion in BALB / C mice using a different CD20 mAb than the one used in the current study suppressed the growth of a lung tumor cell line in vivo, indicating that B cells can negatively regulate tumor immunity (145). However, the remaining mature and MZ B cells left behind may have contributed to $\mathrm{T}$ cell activation in the absence of regulatory B10 cells (67). Although the absence of B cells in the current study inhibited the development of tumor immunity, this does not eliminate a potential inhibitory role for regulatory B10 cells during tumor immune responses, but rather indicates that the positive regulatory functions of B cells dominated in this context. Thus, it remains possible that regulatory B10 cells may inhibit immune responses at different stages of tumor progression or in response to different tumors. Thereby, selectively eliminating the negative influence of B10 cells during anti-tumor immune responses may potentiate the development of tumor immunity.

That B cells are required for $\mathrm{T}$ cell activation in the context of tumor immunity may also be applicable to multiple T cell-mediated autoimmune diseases where B cell depletion delays disease onset and reduces disease severity. Nonetheless, the current studies do not suggest or imply that B cell depletion in humans will promote or accelerate non-lymphoid tumor growth. The mouse anti-mouse CD20 mAb used in the current studies depletes the vast majority of mature B cells $(2,39,71)$. Studies of B cell depletion in humans treated with rituximab predominantly focus on blood, where B cells represent $<2 \%$ of total B cells. Thus, sufficient numbers of B cells may persist in human tissues after rituximab treatment to support T cell activation (146). Nonetheless, as with all forms of immunosuppression, cancer screening for patients considering B cell 
depletion therapies and paying attention to cancer rates in patients receiving $\mathrm{B}$ cell depletion therapies is warranted.

In conclusion, B cells are required for optimal cellular immune responses against B16 tumors in vivo, with B cell depletion reducing effector-memory and cytokinesecreting $\mathrm{CD}^{+}$and $\mathrm{CD} 8^{+} \mathrm{T}$ cell generation and the activation and proliferation of tumor Ag-specific $\mathrm{CD}^{+} \mathrm{T}$ cells. These results further confirm that $\mathrm{B}$ cells have significant functions, either as APCs and/or sources of costimulatory molecules in vivo when Ag concentrations are low, as occurs during tumor development. The therapeutic benefits from depleting B cells in autoimmunity are also likely to be attributable to reducing $\mathrm{T}$ cell contributions to disease symptoms. Thereby, these studies argue strongly against multiple previous proposals to augment anti-tumor immunity by depleting B cells and thereby augmenting Th1 responses $(83-85,125,145)$. Conversely, targeting tumor Ags to B cells in addition to DCs is likely to optimize tumor-directed vaccines and immunotherapies $(141,147)$. 


\section{B Lymphocytes Differentially Influence Acute and Chronic Allograft Rejection in Mice}

The following text was slightly modified from its original manuscript, "B Lymphocytes Differentially Influence Acute and Chronic Allograft Rejection in Mice," currently under review at The Journal of Immunology.

\subsection{Introduction}

The role of B lymphocytes during allograft rejection remains unclear since B cells perform multiple functions during immune responses. For example, terminally differentiated plasma cells represent the effector cells of humoral immunity but also secrete pathogenic $\mathrm{Ab}$. B cells also positively regulate cellular immune responses by serving as APCs, and B cell Ag presentation is required for optimal Ag-specific $\mathrm{CD}^{+} \mathrm{T}$ cell expansion, memory formation, and cytokine production $(19,20,27)$. B cells also provide costimulatory signals to T cells through CD80, CD86, and OX40L that are important for optimal $\mathrm{T}$ cell activation $(30,148)$. A population of regulatory B cells (B10 cells) has also been identified that negatively regulates inflammation and immune responses through the production of IL-10 (116). Adoptive transfer of regulatory B10 cells suppresses immune responses and disease in mouse models of DTH, EAE, and lupus $(58,68,116,149)$. Thus, B cells may carry out any or all of these functions during allograft rejection.

A variety of strategies have been employed to manipulate B cells and their $\mathrm{Ab}$ products during allograft rejection in humans and mice. Most studies using genetically B cell-deficient $\mu \mathrm{MT}$ mice have demonstrated that B cells and Ab are not required for acute allograft rejection in various models (150-152), while other reports indicate roles for B cells during allograft rejection $(153,154)$. Even when allograft-specific Ab production is not required for graft rejection, the exogenous addition of allograft-specific 
Ab can induce allograft damage $(150,154,155)$. However, complex issues are associated with studying allograft rejection in $\mu \mathrm{MT}$ mice, where the immune system develops in the complete absence of B cells. $\mu \mathrm{MT}$ mice have severe immune system abnormalities including a decreased $\mathrm{T}$ cell repertoire and numbers $(34,127)$, a smaller spleen size (127), altered Th1 / Th2 cytokine balance (23), and missing follicular DCs and macrophage populations (37). For this reason, the current study has examined the contributions of B cells to allograft rejection using adult mice that were treated with mAbs to deplete B cells, but with otherwise intact immunity $(71,156)$.

Strategies have been developed for B cell depletion in humans and mice using CD20 and CD19 mAbs (157). In mice, these mAbs deplete B cells in vivo by monocytemediated Ab-dependent cellular cytotoxicity $(71,156)$ without inducing a systemic cytokine release that could alter the function of other immune system components (27). In C57BL / 6 mice, more than 95\% of mature B cells in the blood and primary lymphoid organs are depleted after $2 \mathrm{~d}$ by a single dose of mouse anti-mouse MB20-11 CD20 $\mathrm{mAb}$, with the effect lasting up to eight weeks (39). CD20 mAb depletes mature, GC, and memory B cells, but does not affect plasma cell numbers or serum IgG levels (2). Mouse anti-human CD19 mAbs can deplete B cells in huCD19Tg mice that have otherwise intact immune systems $(102,156)$. Human CD19 expression in huCD19Tg mice parallels CD19 expression in humans (102). Treatment of C57BL / 6 huCD19Tg mice with CD19 mAb depletes pre-B cells and mature B cells, and also reduces basal serum IgM and IgG levels. Since B cell depletion using CD19 mAb represents a potential new therapy for transplantation, huCD19Tg mice were used in this study to directly compare an anti-human CD19 mAb with a potent anti-mouse CD20 mAb that depletes the vast majority of mature B cells.

Since a wide variety of results have been obtained using different mouse models to identify the role of B cells during allograft rejection, this study compared the effects of 
CD20 and CD19 mAb treatment in four distinct allograft rejection models: fully mismatched acute cardiac, chronic renal, and acute skin allografts, and minor-Ag mismatched skin allografts. Using these model systems, B cells and allograft-specific Ab were found to be required for the pathogenesis of chronic kidney allograft rejection, since only CD19 mAb prevented rejection in this model. By contrast, B cells (B10 cells) can exert inhibitory functions during skin allograft rejection because B cell depletion enhanced allograft-specific cellular immune responses and rejection. Thereby, B cells can either positively or negatively regulate allograft rejection depending on the nature of the allograft and the intensity of the rejection response.

\subsection{Results}

\subsubsection{CD20 and CD19 mAbs Effectively Deplete Different B Cell Populations}

Mouse anti-mouse CD20 (MB20-11) and mouse anti-human CD19 (FMC63) mAbs deplete B cells in C57BL / 6 mice and C57BL / 6 huCD19Tg mice, respectively (71, 156). However, C57BL / 6-129 (F1) huCD19Tg mice were utilized for cardiac and renal

allografts in the current studies since their larger size and hybrid vigor enhanced survival during and after allograft surgery. Therefore, B cell depletion with these CD20 and CD19 mAbs was compared side-by-side in C57BL / 6-129 huCD19Tg mice given isotype control, CD20, or CD19 mAb for $7 \mathrm{~d}$.

Within the BM, CD19 mAb treatment decreased B220+ $\mathrm{B}$ cell numbers by $90 \%$ $(p=0.003)$, while CD20 mAb treatment had a small effect (Figure 13A). Both CD20 and CD19 mAb treatments decreased $\operatorname{IgM}^{+} B 220^{\text {hi }}$ mature B cell numbers by $>97 \%(p=0.003)$. Furthermore, CD19 mAb treatment depleted $\operatorname{IgM}^{+} B 220^{\text {low }}$ immature B cells by $65 \%$ $(\mathrm{p}=0.03)$ and $\operatorname{IgM}-\mathrm{B} 220^{\text {low }} \mathrm{CD}^{-}$pre-B cells by $94 \%(\mathrm{p}=0.02 ;$ Table 2$)$. In the periphery, CD20 or CD19 mAb treatment reduced blood B220 cell numbers by $>97 \%(\mathrm{p}=0.001)$, 
spleen $\mathrm{B} 220^{+}$cell numbers by $>78 \%(\mathrm{p} \leq 0.009)$, and LN B220 ${ }^{+}$cell numbers by $>83 \%$ $(\mathrm{p} \leq 0.005$; Figure 13B-D). In the spleen, CD20 mAb treatment depleted $59 \%(\mathrm{p}=0.02)$ of transitional-1, 85\% ( $\mathrm{p}=0.001)$ of transitional-2, and 91\% ( $\mathrm{p}=0.003)$ of MZ B cells. CD19 $\mathrm{mAb}$ depleted $77 \%(\mathrm{p}=0.002)$ of transitional-1, 81\% ( $\mathrm{p}=0.002)$ of transitional-2, and 72\% ( $\mathrm{p}=0.008)$ of MZ B cells. Thus, CD19 mAb treatment depleted pre-B and immature BM B cells in huCD19Tg mice, while the CD20 and CD19 mAbs depleted peripheral B cells similarly. 


\section{A. Bone Marrow}
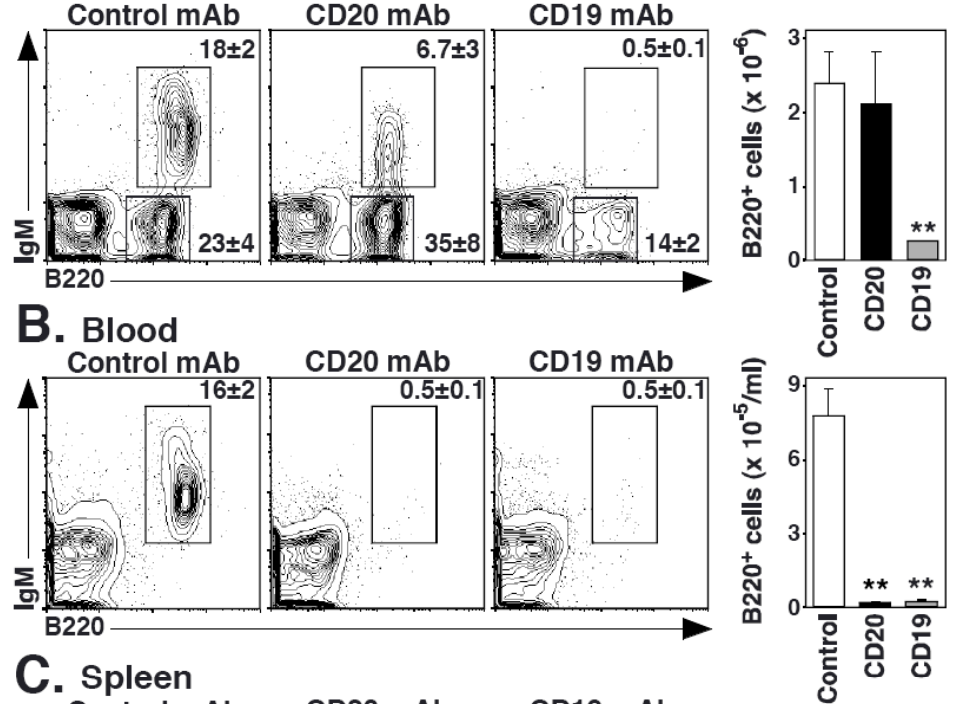

C. Spleen

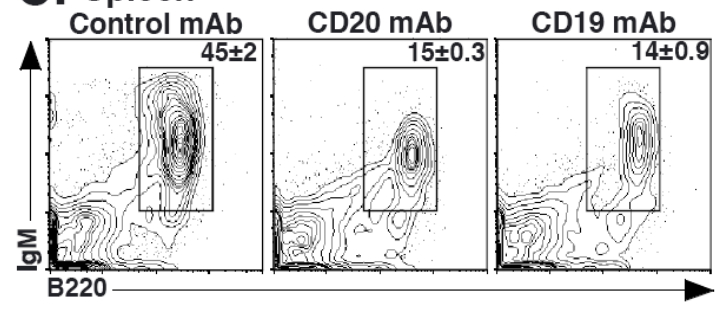

D. Lymph Node
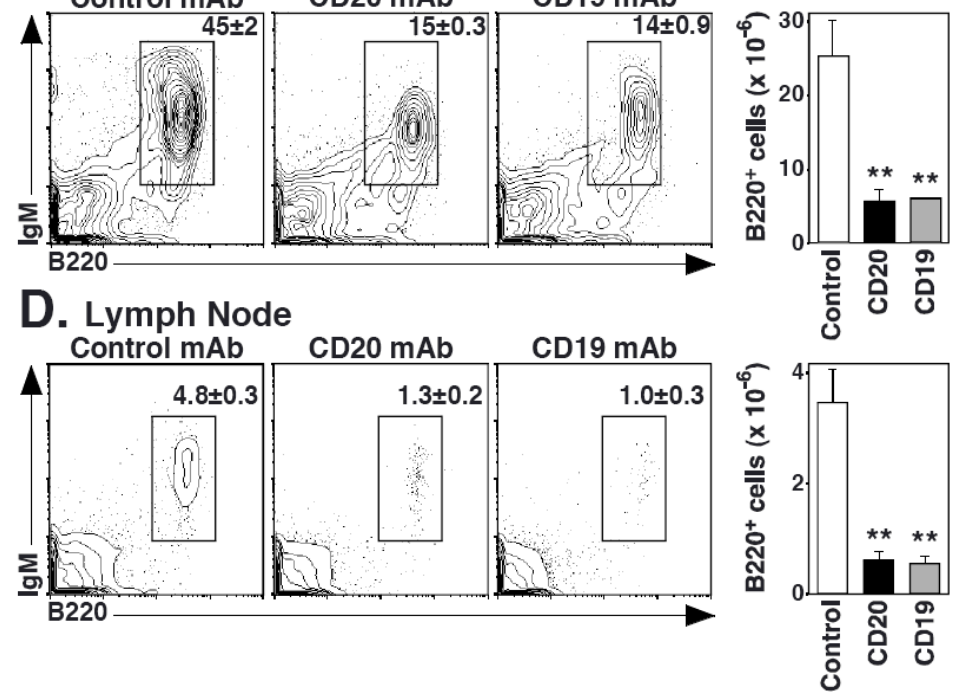

Figure 13. CD20 and CD19 mAbs effectively deplete mature B cells in vivo. C57BL / 6-129 huCD19Tg mice were given control, CD20, or CD19 mAb. B cell numbers within the (A) BM, (B) blood, (C) spleen, and (D) pooled inguinal, axial, and brachial LN were analyzed $7 \mathrm{~d}$ later by immunofluorescence staining with flow cytometry analysis. Representative dot plots are shown, with the mean ( \pm SEM) percentage of $\mathrm{B}_{22} 0^{+} \mathrm{IgM}^{+}$or $\mathrm{B}_{220^{+}} \mathrm{IgM}^{-}$cells indicated. Bar graphs indicate the mean $( \pm \mathrm{SEM}$ ) numbers of $\mathrm{B} 220^{+} \mathrm{B}$ cells contained within each tissue after the indicated treatment $(\mathrm{n}=3$ mice per group) from one of two independent experiments. Significant differences between control and CD20 or CD19 mAb-treated sample means are indicated: ${ }^{* *}, \mathrm{p}<0.01$. 
Table 2. B cell subsets in CD20 and CD19 mAb-treated mice

\begin{tabular}{|c|c|c|c|c|}
\hline Tissue & Subset & Control $\mathrm{mAb}$ & CD20 mAb & CD19 mAb \\
\hline \multirow[t]{5}{*}{$\mathrm{BM}$} & $\mathrm{B} 220^{+}$ & $2.04 \pm 0.34$ & $1.80 \pm 0.60$ & $0.21 \pm 0.02^{* *, \#}$ \\
\hline & Pro & $0.44 \pm 0.05$ & $0.47 \pm 0.01$ & $0.40 \pm 0.07$ \\
\hline & Pre & $0.81 \pm 0.23$ & $1.12 \pm 0.43$ & $0.05 \pm 0.01^{*}$, \# \\
\hline & Immature & $0.57 \pm 0.10$ & $0.31 \pm 0.13$ & $0.006 \pm 0.002^{* *, \#}$ \\
\hline & Mature & $0.38 \pm 0.07$ & $0.01 \pm 0.005^{* *}$ & $0.004 \pm 0.001^{* *}$ \\
\hline Blood & $\mathrm{B} 220^{+}$ & $7.86 \pm 1.09$ & $0.18 \pm 0.05$ & $0.22 \pm 0.09$ \\
\hline \multirow[t]{5}{*}{ Spleen } & $\mathrm{B} 220^{+}$ & $23.93 \pm 4.58$ & $5.30 \pm 1.50^{* *}$ & $5.63 \pm 0.17^{* \star}$ \\
\hline & $\mathrm{T} 1$ & $3.39 \pm 0.42$ & $1.40 \pm 0.45^{*}$ & $0.78 \pm 0.04^{* *}$ \\
\hline & $\mathrm{T} 2$ & $3.01 \pm 0.38$ & $0.04 \pm 0.02^{* *}$ & $0.57 \pm 0.12^{* *}$ \\
\hline & Marginal zone & $3.15 \pm 0.54$ & $0.28 \pm 0.10^{* *}$ & $0.87 \pm 0.17^{* *}$ \\
\hline & Mature & $16.16 \pm 3.78$ & $3.79 \pm 0.93^{* *}$ & $3.73 \pm 0.31^{* *}$ \\
\hline LN & $\mathrm{B} 220^{+}$ & $0.37 \pm 0.06$ & $0.06 \pm 0.02^{* *}$ & $0.05 \pm 0.01^{* *}$ \\
\hline
\end{tabular}

Naîve huCD19Tg mice were treated with control, CD20, or CD19 mAb, with B cell subset numbers determined by immunofluorescence staining with flow cytometry analysis. B cell subsets were: BM pro-B (B220 $\left.{ }^{\text {low }} \operatorname{IgM}^{-} \mathrm{CD} 43^{+}\right)$, pre-B $\left(\mathrm{B} 220^{\text {low }} \operatorname{IgM}^{-} \mathrm{CD}^{-} 3^{-}\right)$, immature B $\left(\mathrm{B}_{22}{ }^{\text {low }} \operatorname{IgM}^{+}\right)$, and mature $\mathrm{B} \quad\left(\mathrm{B}_{2} 20^{\text {high }} \mathrm{IgM}^{+}\right)$; spleen mature $\left(\mathrm{B} 220^{+} \mathrm{CD} 24^{+} \mathrm{CD} 21^{+}\right), \mathrm{T} 1 \quad\left(\mathrm{~B} 220^{+} \mathrm{CD} 24^{\text {high }} \mathrm{CD} 21^{-}\right), \quad \mathrm{T} 2\left(\mathrm{~B} 220^{+} \mathrm{CD} 24^{\text {high }} \mathrm{CD} 21^{+}\right)$, and marginal zone $\left(B 220^{+} \mathrm{CD} 21^{\text {high }} \mathrm{CD} 1 \mathrm{~d}^{+}\right)$. Values $\left( \pm\right.$SEM) indicate cell numbers $\left(\times 10^{-6}\right)$ present in each tissue $7 \mathrm{~d}$ after $\mathrm{mAb}$ treatment ( $\mathrm{n}=3$ mice per group): blood shown as cells $\times 10^{-5} / \mathrm{ml}$, LN as pooled bilateral inguinal, axial, and brachial $\mathrm{LN}$, and BM as pooled femurs. Significant differences between means are indicated: ${ }^{*}, \mathrm{p} \leq 0.05$ vs. control group; ${ }^{* *}, \mathrm{p} \leq 0.01$ vs. control group; ${ }^{\#}, \mathrm{p} \leq 0.05$ vs. CD20 mAb-treated group. 


\subsubsection{CD19 mAb, But Not CD20 mAb Treatment Depletes Serum IgG in Naïve Mice}

CD20 mAb treatment does not alter serum Ig levels in C57BL / 6 mice (2), while CD19 mAb treatment depletes serum IgM, IgG, and autoantibodies (autoAbs) in C57BL / 6 huCD19Tg mice (156). To directly compare the effects of CD20 and CD19 mAbs on serum Ig levels in the same mouse line, C57BL / 6 huCD19Tg mice were treated with control, CD20, or CD19 mAb, with serum harvested over a 6 mo time period. Serum Ig levels increase in huCD19Tg mice due to enhanced CD19 signaling, with these mice producing autoAbs and becoming hypergammaglobulinemic with age (103). After 1 mo of CD20 or CD19 mAb treatment, serum IgM levels decreased by $83 \%$ and $75 \%$, respectively $(\mathrm{p}<0.00001)$, relative to their levels before $\mathrm{mAb}$ administration (Figure 14A). After 1 mo of CD19 mAb treatment, serum IgG levels decreased by $78 \%$ $(\mathrm{p}<0.00001)$, while CD20 mAb treatment only prevented the increase in IgG levels observed in control mAb-treated mice (Figure $14 \mathrm{~B}, \mathrm{p}=0.02$ relative to control $\mathrm{mAb}$ ). Serum Ig levels in mAb-treated mice began to increase after 2 mo, when B cells returned to the periphery $(39,71)$.

The effect of CD20 and CD19 mAb treatments on IgG autoAb levels was also determined in huCD19Tg mice. After 1 mo of CD19 mAb treatment, anti-ssDNA and anti-histone IgG autoAb levels were reduced by 75\% ( $\mathrm{p}=0.0002)$ and 57\% $(\mathrm{p}<0.0001)$, respectively, while these IgG autoAbs were maintained in CD20 mAb-treated mice (Figure 14C-D). Thus, CD19 mAb treatment significantly reduced normal and autoreactive serum IgG levels, while CD20 mAb treatment only delayed age-associated increases in $\mathrm{Ab}$ and auto $\mathrm{Ab}$ production. 

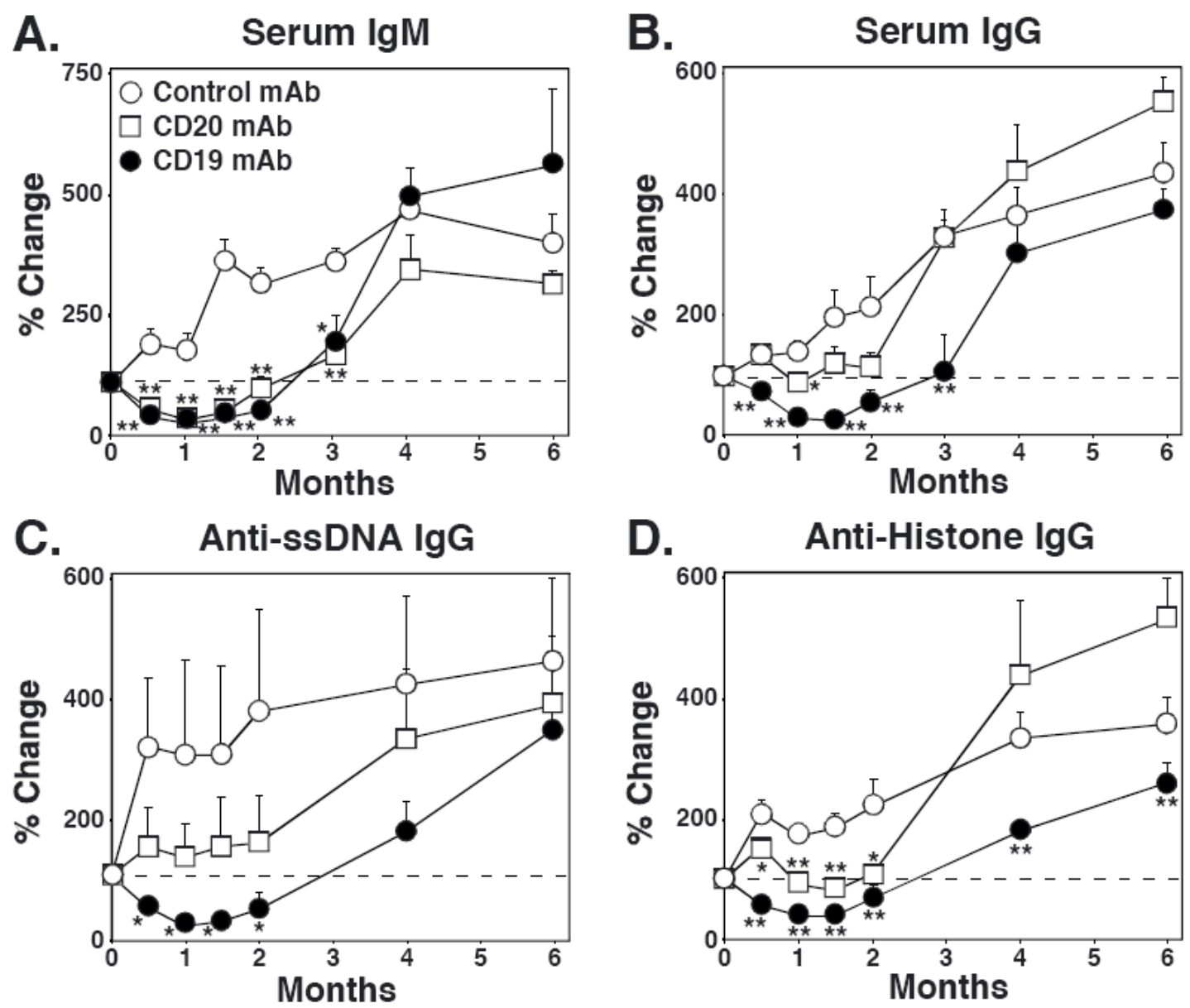

Figure 14. CD19 mAb treatment depletes serum IgG, while CD20 mAb treatment does not. Two mo-old C57BL / 6 huCD19Tg mice were given control, CD20, or CD19 $\mathrm{mAb}$ on day 0 , with serum harvested at the indicated time points for analysis by ELISA. Values represent the mean $( \pm$ SEM) percentage changes in total $\operatorname{IgM}(A)$ or total IgG (B) concentrations, or relative ODs for ssDNA-specific (C) or histone-specific (D) IgG from individual sera relative to day 0 values within the indicated treatment groups $(n=4-5$ mice per group). All values were normalized to 0 for day 0 , with horizontal dashed lines indicating $100 \%$ values. Significant differences between sample means relative to the control mAb treatment group are indicated: ${ }^{*}, \mathrm{p}<0.05 ;{ }^{* *}, \mathrm{p}<0.01$. 


\subsubsection{CD19 mAb Treatment Depletes Ab-Secreting Plasma Cells}

Because CD19 mAb treatment reduced serum IgG levels while CD20 mAb did not, the effect of CD20 and CD19 mAb treatment on plasma cell numbers was assessed. Plasmablasts and plasma cells express CD138, a cell surface marker used for ASC identification $(158,159)$. Short-lived plasmablasts $\left(\mathrm{CD} 138^{\text {hi }} \mathrm{BrdU}^{+}\right)$and long-lived plasma cells (CD138 ${ }^{\text {hi }}$ BrdU') can be phenotypically identified using CD138 staining in combination with in vivo BrdU labeling (160). Therefore, huCD19Tg mice were fed BrdU for $10 \mathrm{~d}$ before treatment with CD19, CD20, or control mAbs, with spleen and BM cells assessed for CD138 and BrdU staining $7 \mathrm{~d}$ later. CD19 mAb treatment depleted 80\% and $58 \%(\mathrm{p} \leq 0.0009)$ of $\mathrm{BM} \mathrm{CD} 138^{\mathrm{hi}} \mathrm{BrdU}^{+}$and $\mathrm{CD} 138^{\mathrm{hi}} \mathrm{BrdU}^{-}$cells, respectively (Figure 15A). CD20 mAb treatment only depleted 33\% ( $\mathrm{p}=0.04)$ of BM CD138 ${ }^{\text {hi }} \mathrm{BrdU}^{+}$ plasmablasts, with no significant effect on $\mathrm{CD}_{138^{\mathrm{hi}}} \mathrm{BrdU}^{-}$plasma cells. Spleen $\mathrm{CD} 138{ }^{\mathrm{hi}} \mathrm{BrdU}^{+}$and $\mathrm{CD} 1388^{\mathrm{hi}} \mathrm{BrdU}^{-}$cells were decreased by $89 \%(\mathrm{p}=0.02)$ and $79 \%$ ( $p=0.002)$, respectively, following CD19 mAb treatment. CD20 mAb treatment depleted splenic $\mathrm{CD} 138^{\mathrm{hi}} \mathrm{BrdU} \mathrm{U}^{+}$plasmablasts and CD138 ${ }^{\mathrm{hi}} \mathrm{BrdU}^{-}$plasma cells by $37 \%$ (not significant) and 54\% ( $\mathrm{p}=0.02)$, respectively. Therefore, CD20 mAb treatment had limited effects on long-lived BM or splenic plasma cells, while CD19 mAb treatment depleted the majority of both short-lived plasmablasts and long-lived plasma cells in the BM and spleen.

The effect of mAb treatment on IgM and IgG ASC numbers was assessed directly in huCD19Tg mice $7 \mathrm{~d}$ after treatment with control, CD20, or CD19 mAb. In the BM, CD19 mAb treatment decreased IgM ASC numbers by 69\% $(\mathrm{p}=0.03)$ and IgG ASC numbers by $66 \%(\mathrm{p}=0.002)$ (Figure 15B). Splenic IgM and IgG ASC numbers were reduced by $63 \%$ and $68 \%(\mathrm{p} \leq 0.005)$, respectively. B contrast, CD20 mAb treatment had 
no effect on ASC numbers in either tissue. Thus, only CD19 mAb treatment depleted both IgM and IgG ASCs.

Cell surface CD20 is downregulated during plasma cell differentiation in humans and mice $(2,161)$, while CD19 loss occurs later during human plasma cell development (162). Whether CD20 and CD19 mAbs differentially deplete mouse plasmablasts due to prolonged cell surface molecule expression on $\mathrm{B} 220^{+} \mathrm{CD} 138^{\text {hi }}$ plasmablasts or more differentiated $\mathrm{B} 220^{\mathrm{lo}} \mathrm{CD} 138^{\mathrm{hi}}$ plasmablasts in the spleen and BM was assessed (Figure 15C). Compared to splenic B220 ${ }^{+}$CD138 follicular B cells, CD20 expression was decreased by $50 \%$ on splenic $\mathrm{B} 220^{\mathrm{lo}} \mathrm{CD} 138^{\mathrm{hi}}$ plasmablasts $(\mathrm{p}<0.00001)$ and by $62 \%$ on $\mathrm{BM}$ B220 ${ }^{\mathrm{lo}} \mathrm{CD} 138^{\mathrm{hi}}$ plasmablasts $(\mathrm{p}<0.00001)$. By contrast, CD19 expression was increased by 2.7-fold and 2.2-fold $(\mathrm{p}<0.0001)$, respectively, on splenic and BM B220 ${ }^{+}$CD $138^{\text {hi }}$ plasmablasts when compared to splenic follicular B cells. CD19 expression subsequently decreased so that spleen $B 220{ }^{\text {low }} C D 138^{\text {hi }}$ plasmablasts and follicular B cells expressed similar CD19 densities, while BM B220 ${ }^{\text {low }}$ CD $138^{+}$ plasmablast densities ranged between 70-100\%. Thus, while CD20 levels decreased during plasma cell differentiation, CD19 expression remained high on plasmablasts. 
A.

Bone Marrow
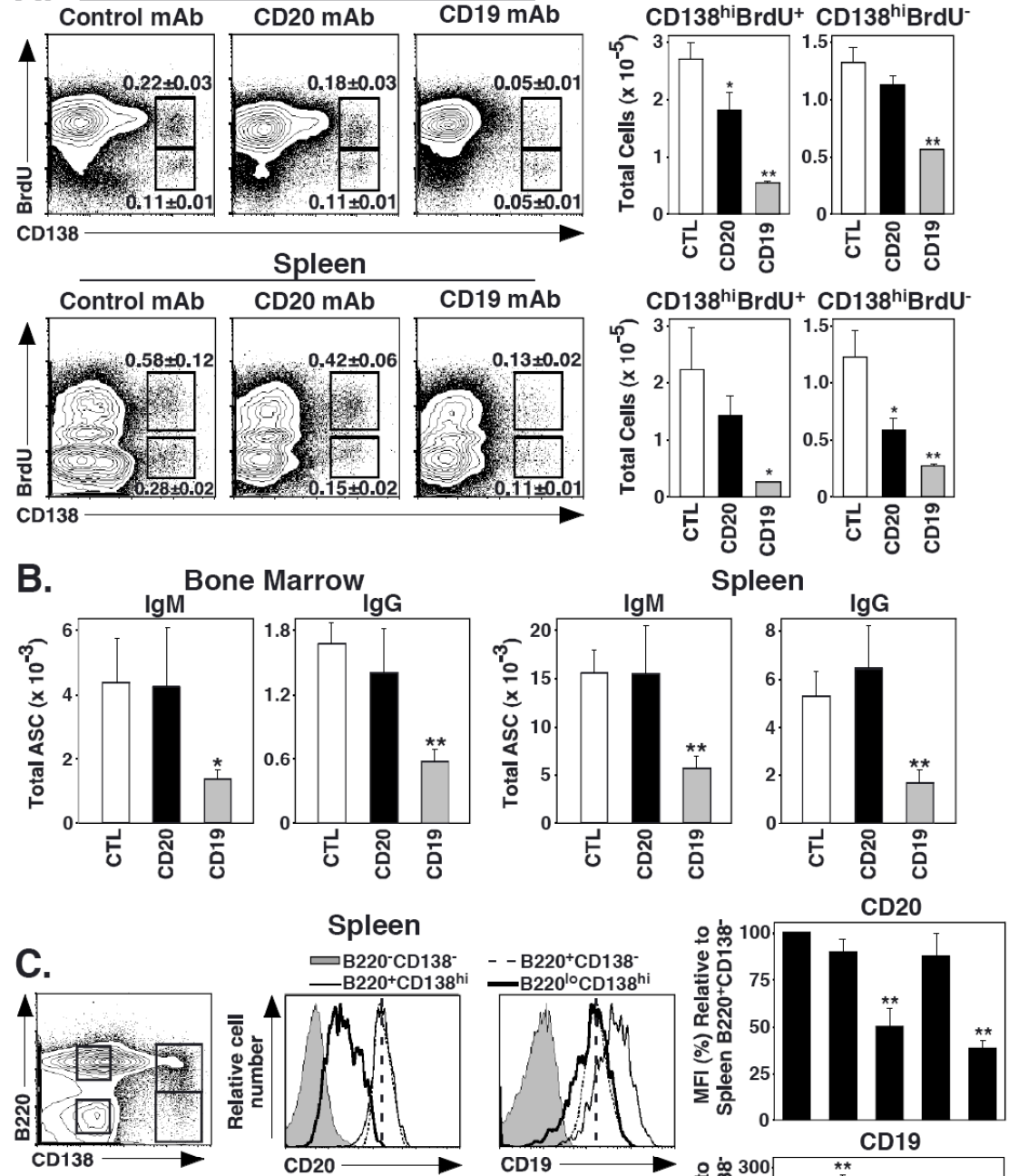

Bone Marrow

$=\mathrm{B} 220^{-\mathrm{CD} 138-}$
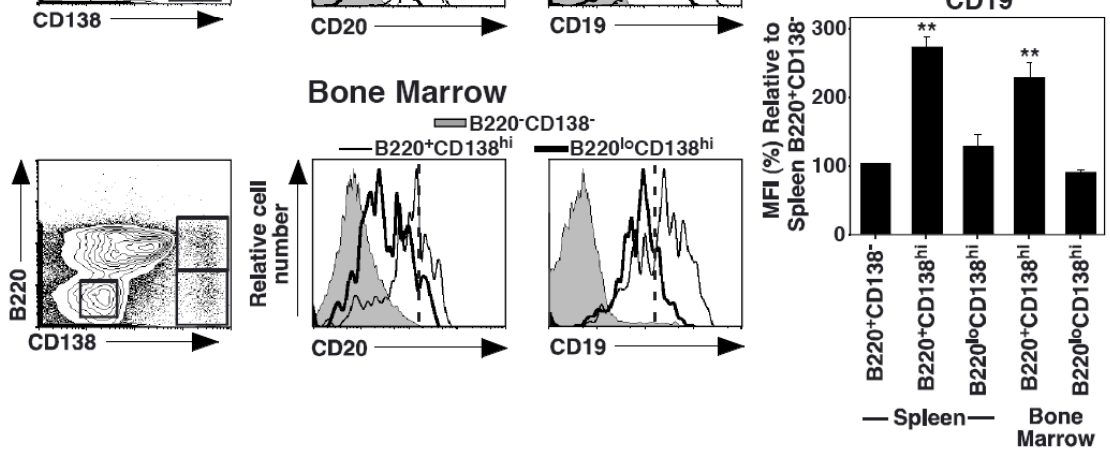

Figure 15. CD19 mAb treatment depletes Ab-secreting plasma cells. (A) CD19 mAb depletes both short-lived and long-lived CD138 ${ }^{\text {hi }}$ plasma cells. C57BL / 6-129

huCD19Tg mice were fed BrdU for $7 \mathrm{~d}$ prior to and following control, CD20, or CD19 $\mathrm{mAb}$ treatments, with BM and spleen cells analyzed for surface CD138 and intracellular BrdU staining on day 7. Dot plots show mean ( \pm SEM) percentages of CD138 ${ }^{\text {hi }} \mathrm{BrdU}^{+}$ (short-lived plasma cells) and CD138 ${ }^{\text {hi }} \mathrm{BrdU}^{-}$(long-lived plasma cells). Bar graphs indicate mean numbers of $\mathrm{CD} 138^{\mathrm{hi}} \mathrm{BrdU}^{+}$and $\mathrm{CD} 138^{\mathrm{hi}} \mathrm{BrdU}^{-}$cells from mice receiving 
Figure 15 (Continued) the indicated treatment ( $\mathrm{n}=8$ mice per group). (B) C57BL $/ 6$ huCD19Tg mice were given control, CD20, or CD19 mAb, with BM and spleen Absecreting cell (ASC) numbers determined $7 \mathrm{~d}$ later by ELISPOT analysis. Bar graphs indicate mean $( \pm$ SEM) numbers of $\operatorname{IgM}$ and IgG ASCs $(n=4$ mice per group). (C) CD19 expression is maintained on CD138 $8^{+}$plasmablasts, while CD20 expression decreases. $\mathrm{BM}$ and spleen cells from naive C57BL / 6-129 huCD19Tg mice were co-stained for CD138, B220, CD20, and CD19. Representative dot plots (left panels) indicate the $\mathrm{B} 220^{-} \mathrm{CD} 138^{-}, \mathrm{B} 220^{+} \mathrm{CD} 138^{-}, \mathrm{B} 220^{+} \mathrm{CD} 138^{\text {hi }}$, and $\mathrm{B} 220^{\mathrm{lo}} \mathrm{CD} 138^{\text {hi }}$ cell populations that were analyzed for CD20 and CD19 expression (right panels). Vertical dashed lines indicate the MFI of CD20 or CD19 expression by splenic B220 ${ }^{+}$CD138 ${ }^{-}$B cells. Bar graphs indicate mean $( \pm$ SEM) levels of CD20 or CD19 expression relative to spleen $\mathrm{B} 220^{+} \mathrm{CD} 138^{-} \mathrm{B}$ cells. Background staining MFI values $(<10)$ were subtracted from each respective population before normalization to splenic $\mathrm{B} 220^{+} \mathrm{CD} 138^{-}$cells. Results from 3 individual experiments ( $n=3$ mice in each experiment) were pooled. (A-C) Significant differences between sample means relative to the control $\mathrm{mAb}$ treatment $(\mathrm{A}-\mathrm{B})$ or to the B220 ${ }^{+} \mathrm{CD} 1388^{-}$population $(\mathrm{C})$ are indicated: ${ }^{*}, \mathrm{p}<0.05 ;{ }^{*}, \mathrm{p}<0.01$. 


\subsubsection{CD19 mAb Treatment Inhibits Graft-Specific IgG Generation during Cardiac Allograft Rejection}

The contributions of B cells and plasma cells to solid organ allograft rejection were assessed in an acute cardiac allograft model where C57BL / 6-129 mice normally

reject fully MHC-mismatched hearts from BALB / C-DBA/ 2 (F1) mice within 7-9 d (163). huCD19Tg mice were treated with control, CD20, or CD19 mAb $7 \mathrm{~d}$ before the mice received heterotopic DBA / 2 cardiac allografts, with graft survival monitored daily. Grafts in all mice were rejected within 7 to $9 \mathrm{~d}$, with no significant differences between groups (control mAb, 7.8 $\pm 0.2 \mathrm{~d}$; CD20 mAb, $8 \pm 0.6 \mathrm{~d}$; CD19 mAb, $6.8 \pm 0.3 \mathrm{~d}$; Figure 16A). However, CD19 mAb treatment inhibited the generation of serum allograftspecific IgM and IgG Abs that were reactive with the DBA/2-derived P815 cell line (Figure 16B). B cell depletion with CD20 and CD19 mAbs decreased allograft-specific IgM levels by $56 \%$ and $71 \%$, respectively $(\mathrm{p} \leq 0.006)$. CD19 mAb treatment decreased allograft-specific IgG levels by $83 \%$ ( $\mathrm{p}=0.004)$, while CD20 mAb treatment had no effect. Thus, CD19 mAb treatment did not affect acute cardiac allograft rejection, but significantly reduced allograft-specific IgG Ab responses. 


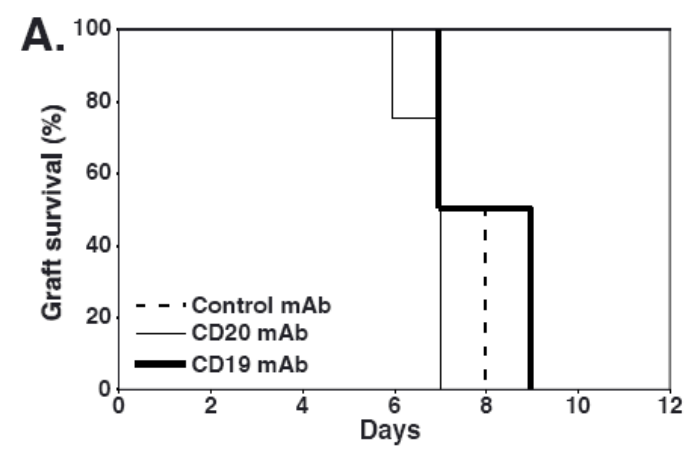

B.

IgM
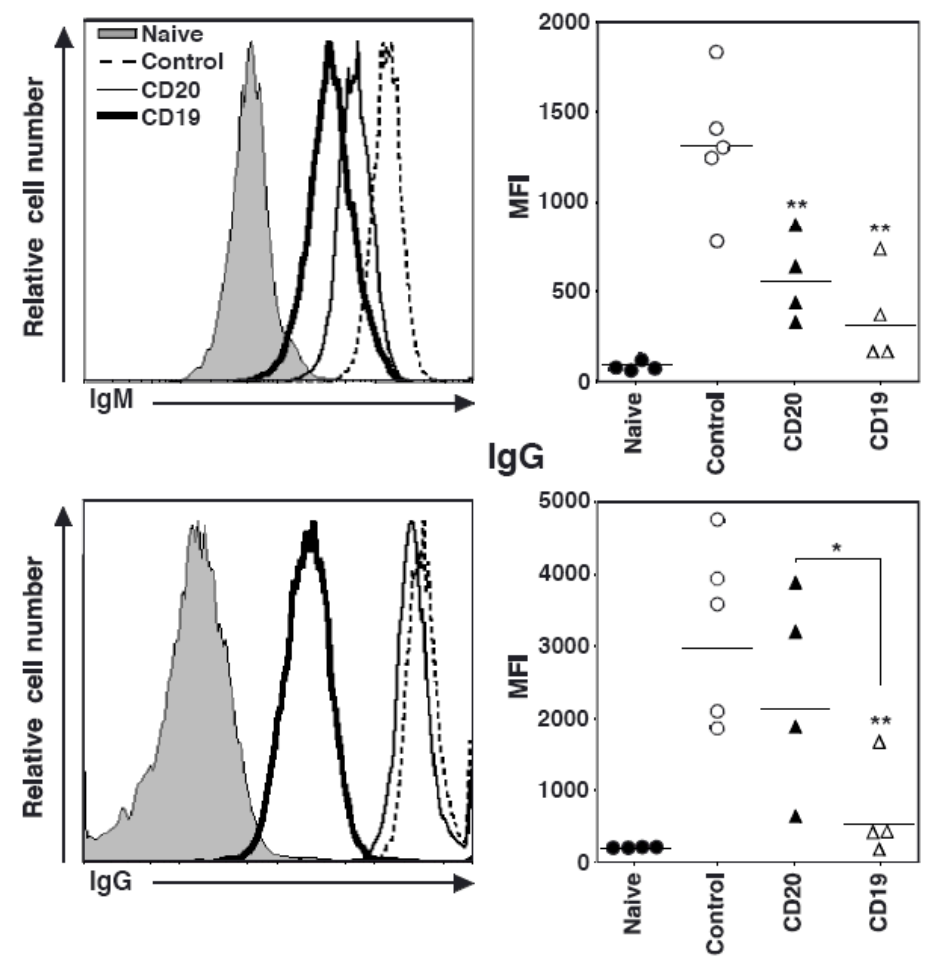

Figure 16. CD19 mAb treatment impairs allograft-specific IgG production. C57BL / 6129 huCD19Tg mice were given control, CD20, or CD19 mAb 7 d before receiving a fully MHC-mismatched DBA / 2 heterotopic cardiac allograft. (A) Allograft survival following control ( $n=5$, dashed line), CD20 ( $n=4$, solid line), or CD19 ( $n=4$, heavy solid line) mAb treatment. (B) Serum was collected from mice in (A) $14 \mathrm{~d}$ after grafting and analyzed for DBA / 2-specific IgM (top panels) and IgG (bottom panels) by indirect immunofluorescence staining with flow cytometry analysis. Flow cytometry histograms (left panels) show representative IgM and IgG staining of DBA/2-derived P815 cells with serum from control (dashed line), CD20 (solid line), and CD19 (heavy solid line) mAb-treated mice, and untransplanted naïve mice (shaded). Values (right panels) represent the MFI of stained cells from individual mice that received the indicated treatment. Horizontal bars indicate mean MFI values for each group. Significant differences between control and CD20 or CD19 mAb sample means, or between specified means, are indicated: ${ }^{*}, \mathrm{p}<0.05 ;{ }^{* *}, \mathrm{p}<0.01$. 


\subsubsection{CD20 mAb Treatment Impairs Skin Allograft-Specific IgG Generation}

Since CD20 mAb treatment did not affect acute rejection of a directly vascularized organ allograft, its effects on fully MHC-mismatched tissue allograft rejection were assessed. Wild type C57BL / 6 mice were treated with control or CD20 mAb $7 \mathrm{~d}$ before receiving fully MHC-mismatched full-thickness BALB / C skin grafts. Grafts were rejected in both the control and CD20 mAb-treated groups, with mean survival times of $11.0 \pm 0.2$ and $10.7 \pm 0.2 \mathrm{~d}$, respectively (Figure 17A). However, BALB / C-specific IgM was reduced by 68\% (p=0.02) in CD20 mAb-treated mice $14 \mathrm{~d}$ after skin grafting (21 d after mAb injection) in comparison with control mAb-treated mice, and graft-specific IgG levels were decreased by 75\% ( $\mathrm{p}=0.005)$ (Figure 17B). Thus, CD20 mAb treatment had no effect on acute skin allograft rejection, but inhibited allograft-specific IgG generation. Because CD20 mAb treatment was sufficient to block IgG responses in the skin allograft model, the effect of CD19 mAb treatment on skin allograft rejection was not assessed.

\subsubsection{CD20 mAb Treatment Accelerates Minor Ag-Mismatched Skin Allograft Rejection}

The effect of mature $\mathrm{CD} 20^{+} \mathrm{B}$ cell depletion on the rejection of skin allografts expressing a single, defined Ag was assessed to reveal differences in rejection that may not have been seen in the context of the vigorous rejection of fully MHC-mismatched grafts. C57BL / 6 mice were treated with control or CD20 mAb $7 \mathrm{~d}$ before receiving skin grafts from C57BL / 6 transgenic act-mOVA mice that express cell surface-bound OVA protein (110). CD20 mAb-treated mice rejected act-mOVA grafts significantly faster

(mean $14.7 \pm 0.7 \mathrm{~d}$ ) than mice that received control $\mathrm{mAb}(17 \pm 0.7 \mathrm{~d} ; \mathrm{p}=0.019, \log$ rank test; chi square=5.5, $\mathrm{df}=1$; Figure $17 \mathrm{C})$. OVA-specific IgM or IgG responses were not detected in either CD20 or control mAb-treated mice $21 \mathrm{~d}$ after grafting (data not 
shown), reflecting the weak $\mathrm{Ab}$ responses generally observed for minor histocompatibility Ags (22). Thus, mature B cell depletion accelerated the rejection of Ag-mismatched skin grafts.

The effects of CD20 mAb treatment on T cell function were assessed since C57BL / 6 mice effectively reject act-mOVA skin grafts in a TD manner (110). C57BL / 6 mice were given either control or CD20 mAb $6 \mathrm{~d}$ before being given CFSE-labeled Thy $1.1^{+} \mathrm{CD}^{+}$or $\mathrm{CD}^{+} \mathrm{T}$ cells from OVA peptide-specific OT-II or OT-I transgenic mice, respectively $(100,101)$. One d later, all mice received act-mOVA skin grafts. The spleen and peripheral LN draining the site of the skin graft were isolated $5 \mathrm{~d}$ later, with CFSE dilution by Thy $1.1^{+} \mathrm{CD}^{+}$or $\mathrm{CD} 8^{+} \mathrm{T}$ cells analyzed by flow cytometry to quantify $\mathrm{T}$ cell proliferation. Draining LN Thy $1.1^{+} \mathrm{CD} 4^{+}$OT-II cells from CD20 mAb-treated mice diluted CFSE two-fold greater $(p=0.006)$ than control mAb-treated mice (Figure 17D), while splenic OT-II cells from CD20 mAb-treated mice diluted CFSE 1.7-fold greater than control mice (Figure 17E). By contrast, CD8 ${ }^{+}$OT-I cells proliferated similarly in the LN and spleen of both control and CD20 mAb-treated mice receiving act-mOVA skin grafts (data not shown). Thus, B cell depletion enhanced CD4 ${ }^{+} \mathrm{T}$ cell activation and proliferation in response to Ag-mismatched tissue transplants, which paralleled accelerated graft rejection. 

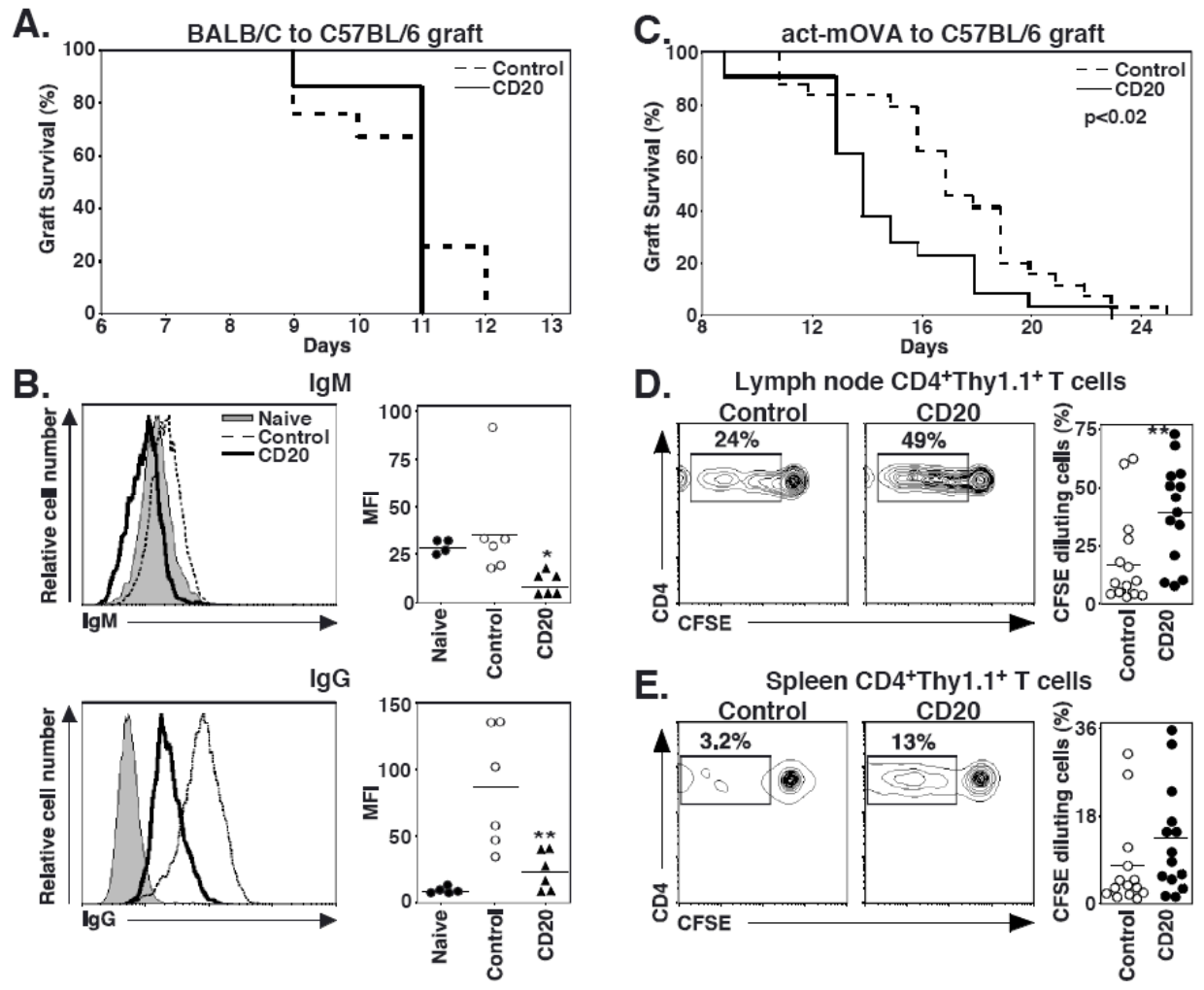

E.

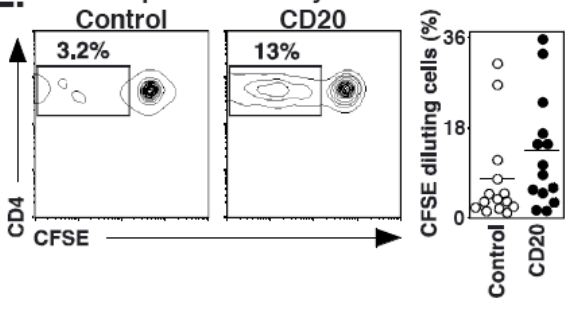

Figure 17. CD20 mAb treatment impairs allograft-specific IgG generation, but accelerates minor histocompatibility-mismatched skin allograft rejection and alloAgspecific $\mathrm{CD4}^{+} \mathrm{T}$ cell proliferation. (A) C57BL/ 6 mice were given control or CD20 mAb $7 \mathrm{~d}$ before receiving BALB / C skin allografts. The graph shows allograft survival following control (dashed line; $n=12$ ) and CD20 (solid line; $n=7$ ) mAb treatment. (B) Serum was collected from mice in (A) $14 \mathrm{~d}$ after grafting and analyzed for BALB / Cspecific IgM (top panels) and IgG (bottom panels) by indirect immunofluorescence staining with flow cytometry analysis. Flow cytometry histograms (left panels) show representative IgM and IgG staining of BALB / C-derived AG8 cells with serum from control (dashed line) and CD20 (solid line) mAb-treated mice, and untransplanted naïve (shaded) mice. Values (right panels) represent the MFI of stained cells from individual mice. Horizontal bars indicate mean MFI values for the group. (C) C57BL / 6 mice were given control or CD20 mAb $7 \mathrm{~d}$ before receiving skin allografts from actmOVA transgenic mice. The graph shows allograft survival following control (dashed line; $\mathrm{n}=24$ ) and CD20 (solid line; $\mathrm{n}=21$ ) $\mathrm{mAb}$ treatment. (D-E) C57BL / 6 mice were given control or CD20 mAb $6 \mathrm{~d}$ before receiving CFSE-labeled Thy $1.1^{+} \mathrm{CD} 4^{+} \mathrm{T}$ cells from OT-II mice. One $d$ later, the mice received Act-mOVA skin grafts. (D) Draining LN and (E) spleen lymphocytes were isolated $5 \mathrm{~d}$ later, with CD4 expression and CFSE dilution of Thy $1.1^{+} \mathrm{T}$ cells assessed by immunofluorescence staining with flow cytometry analysis. Representative CFSE versus cell surface CD4 staining for Thy $1.1^{+}$cells is shown, with the percentages of CFSE-diluted $\mathrm{CD} 4^{+}$cells within each gate indicated as a fraction of total CD4 ${ }^{+}$Thy $1.1^{+} \mathrm{T}$ cells. Values (right panels) represent the percentage of CFSEdiluted cells from individual control mAb- and CD20 mAb-treated mice. Horizontal bars indicate mean values for groups. Significant differences between control $\mathrm{mAb}$ and CD20 mAb sample means are indicated: ${ }^{*}, \mathrm{p}<0.05 ;{ }^{* *}, \mathrm{p}<0.01$. 


\subsubsection{CD19 mAb Treatment Prevents Chronic Renal Allograft Rejection}

A renal allograft model was used to assess the contributions of B cells and plasma cells to chronic allograft rejection (164). huCD19Tg mice were treated with control, CD20, or CD19 mAb 7 d before being transplanted with a kidney from a fully MHC-mismatched DBA / 2 donor. After a bilateral native nephrectomy, graft rejection was determined by assessing mouse survival. Kidney allografts in mice survive for prolonged periods without immunosuppression, but develop pathological features consistent with chronic allograft rejection in humans. Graft survival was similar in control and CD20 mAb-treated mice (mean survival time for control mAb, 54 $\pm 8 \mathrm{~d}$; CD20 mAb, 53 $\pm 9 \mathrm{~d}$ ), with only $20-22 \%$ of mice surviving $>100 \mathrm{~d}$ (Figure $18 \mathrm{~A}$ ). However, CD19 mAb treatment significantly enhanced survival ( $84 \pm 9$ d) when compared to control $(\mathrm{p}=0.023, \log$ rank test; chi square $=5.2, \mathrm{df}=1)$ or $\mathrm{CD} 20(\mathrm{p}=0.035$, log rank test; chi square $=4.4, \mathrm{df}=1) \mathrm{mAb}$-treated mice, with $67 \%$ of CD19 mAb-treated mice surviving for $>100 \mathrm{~d}$.

Whether CD20 or CD19 mAb treatment affected the development of IgM and IgG allograft-specific $\mathrm{Ab}$ responses was assessed in separate groups of allografted mice by harvesting serum $21 \mathrm{~d}$ after kidney grafting. Pre-treatment with both CD20 and CD19 mAbs decreased serum allograft-specific IgM levels by 57\% and 77\%, respectively $(\mathrm{p} \leq 0.002$; Figure 18B). Allograft-specific IgG levels were decreased by $91 \%$ to background levels in CD19 mAb-treated mice $(p=0.005)$ and decreased by $67 \%$ $(\mathrm{p}=0.02)$ by CD20 mAb treatment (Figure 18B). Thus, CD19 mAb treatment abrogated allograft-specific IgG responses while CD20 mAb only inhibited allograft-specific IgG responses.

The severity of renal morphologic abnormalities was assessed $21 \mathrm{~d}$ after transplantation by H\&E, PAS, and Masson's Trichrome staining to determine whether 
enhanced allograft survival in CD19 mAb-treated mice was associated with reduced kidney pathology. In control mAb-treated allografts, the predominant finding was the intense infiltration of inflammatory cells within interstitial regions of the kidney (Table 3), which was less severe in CD20 (32\% decrease, $\mathrm{p}=0.004)$ and CD19 (43\%, $\mathrm{p}=0.0006)$ mAb-treated mice (Figure 19A, data not shown). Tubular pathologic changes (tubulitis, the defining feature of acute renal allograft rejection) were most severe in control mice, but were decreased (39\% decrease, $\mathrm{p}=0.01)$ in CD20 mAb-treated mice. However, there was a $70 \%$ decrease $(p=0.001)$ in tubular pathology in CD19 mAb-treated mice when compared with control mice. Tubular pathology was also decreased by $51 \%$ in CD19 mAb-treated mice when compared to mice receiving CD20 mAb ( $\mathrm{p}=0.04$; Table 3$)$. In general, glomerular pathologic changes were variable and less pronounced. An overall histologic score for each kidney was obtained by summing the individual grades for the glomeruli, tubules, interstitium, and vasculature pathologies (Figure 19A, Table 3). Kidneys from control mAb-treated mice showed the highest overall pathologic score, while the mean scores for kidneys from CD20 or CD19 mAb-treated mice were decreased by 33\% ( $\mathrm{p}=0.03)$ and $47 \%(\mathrm{p}=0.006)$, respectively, when compared to control mice.

Complement fragment $\mathrm{C} 4 \mathrm{~d}$ deposition in peritubular capillaries is a hallmark of Ab-mediated kidney allograft rejection and is closely associated with chronic rejection in renal transplant patients (165). Therefore, C4d deposition was assessed by immunohistochemistry in the allografted kidneys of control, CD20, and CD19 mAbtreated mice 3 wks after transplant surgery. Virtually all of the capillaries in mice receiving control or CD20 mAb exhibited an intense homogenous pattern of mural C4d staining, while most of the CD19 mAb-treated mice showed only weak homogeneous deposition of C4d within the capillary walls (Figure 19B). The mean intensity of C4d deposition was decreased by $30 \%(\mathrm{p} \leq 0.008)$ in the peritubular capillaries of mice 
receiving CD19 mAb when compared to control mAb- and CD20 mAb-treated mice. Similarly, intense homogenous IgG staining was seen within most of the peritubular capillary walls of kidneys from control or CD20 mAb-treated mice, while only occasional capillaries in CD19 mAb-treated mice showed weak mural IgG staining (Figure 19D). The mean intensity of capillary IgG deposition was decreased by $24 \%$, $(\mathrm{p} \leq 0.02)$ in both CD20 and CD19 mAb-treated mice when compared with control mice. Intense intraluminal and mural IgM staining of peritubular capillaries was also seen in kidneys from control mAb-treated mice, while weak to moderately intense staining of many peritubular capillary walls was seen in kidneys from mice receiving CD20 mAb (Figure 19C). Most of the vessels in kidneys from CD19 mAb-treated mice were devoid of IgM staining and only weak focal staining of rare peritubular capillaries was seen. The intensity of IgM staining was decreased by 33\% ( $p=0.004$; Figure 19C) in both CD20 and CD19 mAb-treated mice when compared with control mice. Thus, CD19 mAb treatment inhibited C4d, IgG, and IgM deposition in allografted kidneys. 

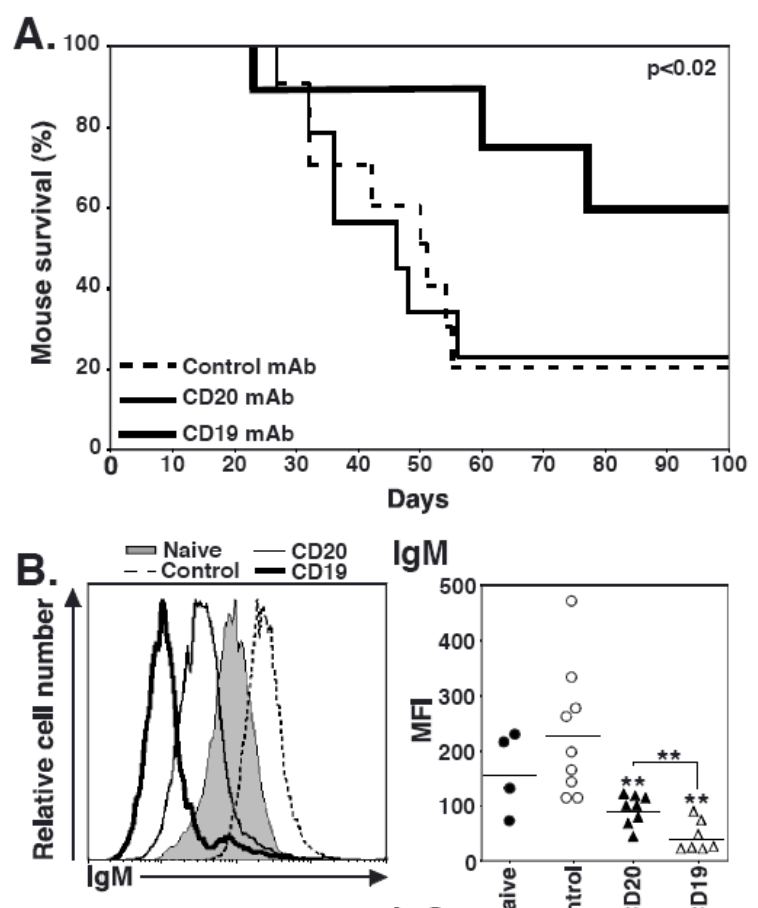

$\lg M$
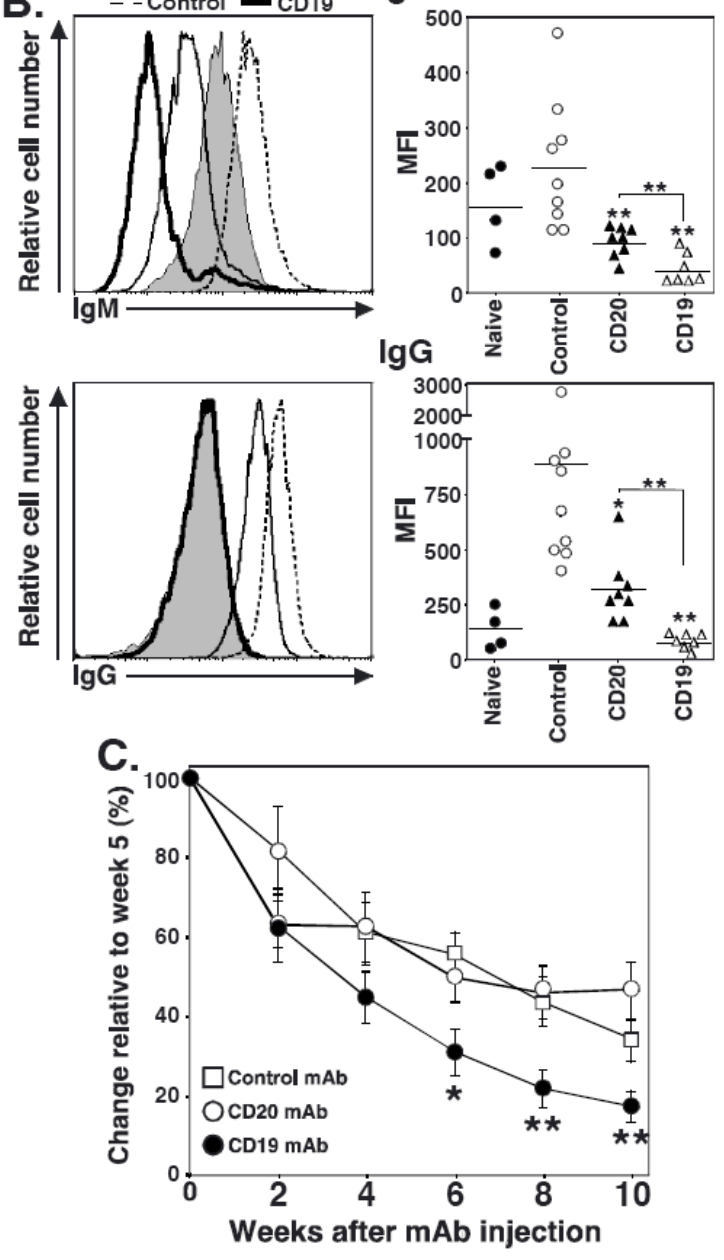

Figure 18. CD19 mAb treatment prevents renal allograft rejection, impairs allograftspecific IgG generation, and reduces pre-existing serum IgG levels. C57BL / 6-129 huCD19Tg mice were given control, CD20, or CD19 mAb $7 \mathrm{~d}$ before receiving a DBA / 2 renal allograft. A nephrectomy was performed on the remaining kidney 1-3 d following allografting, and mouse survival was monitored daily as a measure of allograft rejection. (A) Allograft survival following control ( $\mathrm{n}=9$, dashed line), CD20 ( $\mathrm{n}=8$, solid line), or 
Figure 18 (Continued) CD19 ( $n=7$, heavy solid line) mAb treatments. (B) Serum was collected from mice $21 \mathrm{~d}$ after grafting and analyzed for DBA / 2-specific IgM (top panels) and IgG (bottom panels) by indirect immunofluorescence staining with flow cytometry analysis. Flow cytometry histograms (left panels) show representative IgM and IgG staining of P815 cells with serum from control (dashed line), CD20 (solid line), and CD19 (heavy solid line) mAb-treated mice, and untransplanted naïve (shaded) mice. Values (right panels) represent the results from individual mice that received the indicated treatment. Horizontal bars indicate mean MFI values for groups. (C)

C57BL / 6-129 huCD19Tg mice were immunized with DBA/ 2 splenocytes at week 0 and boosted at week 2. Mice were then randomized and given control, CD20, or CD19 mAb at week 5. Serum was harvested at the indicated time points, with DBA/2-specific IgG measured by indirect immunofluorescence staining with flow cytometry analysis. Values represent the mean percent change in MFI relative to week 5 ( $n=4-5$ mice per group). Significant differences between control and CD20 or CD19 mAb sample means, or between specified means, are indicated: ${ }^{*}, \mathrm{p}<0.05 ;{ }^{* *}, \mathrm{p}<0.01$. 


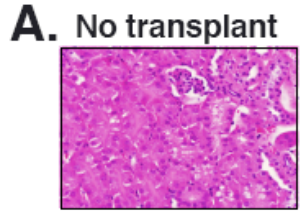

CD20

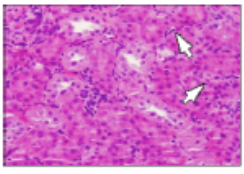

B. No transplant

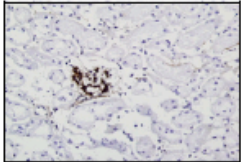

CD20

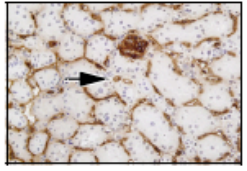

C. No transplant

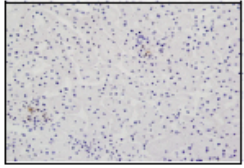

CD20

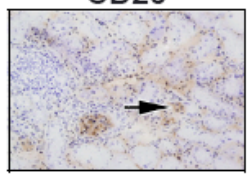

D. No transplant

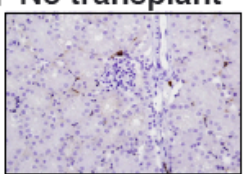

CD20

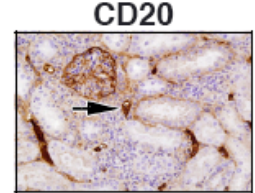

Control

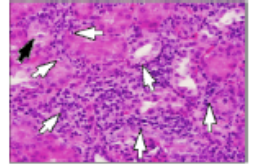

CD19

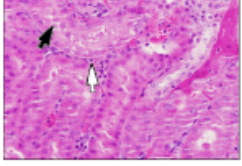

Control

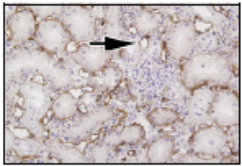

CD19

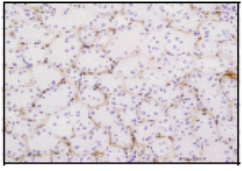

Control

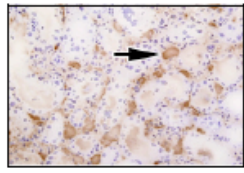

CD19

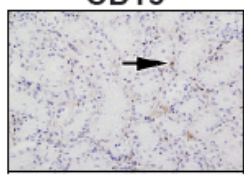

Control

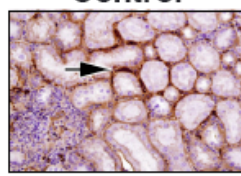

CD19

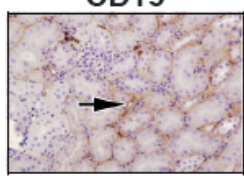

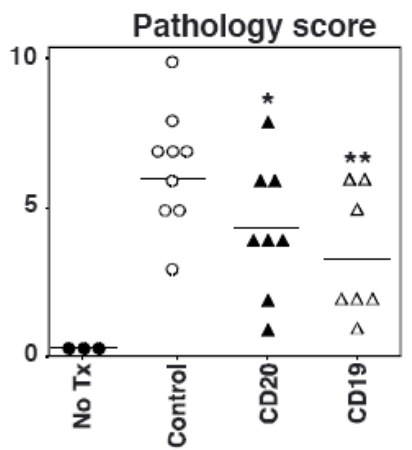
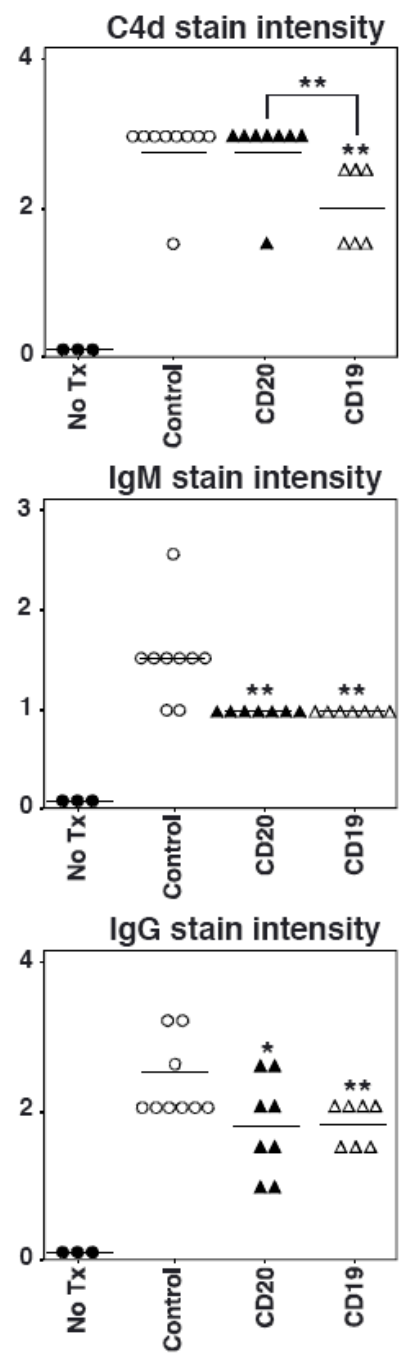

Figure 19. CD19 $\mathrm{mAb}$ treatment reduces renal injury and $\mathrm{C} 4 \mathrm{~d}$ deposition

following renal allografting. C57BL / 6-129 huCD19Tg mice were given control, CD20, or CD19 mAb $7 \mathrm{~d}$ before receiving a fully MHC-mismatched DBA / 2 renal allograft as in figure 20. Mice were sacrificed $21 \mathrm{~d}$ after grafting for donor kidney analysis. (A)

Representative H\&E stained samples (left panels), with tubulitis (white arrows) and foci 
Figure 19 (Continued) of acute tubular necrosis (black arrows) indicated.

Histopathologic scores (right panel) for individual kidneys from mice that received the indicated treatments. Horizontal bars indicate mean overall scores for each group. (B-D) Kidney sections (left panels) from mice treated as in (A) were stained for C4d (B), IgM (C), and IgG (D) deposition, with representative samples shown. Black arrows indicate representative peritubular capillaries. (Right panel) Values represent the staining

intensity score for peritubular capillaries from individual kidneys of mice that received the indicated treatment. Horizontal bars indicate mean score values for the group.

Significant differences between control and CD20 or CD19 mAb sample means, or between specified means, are indicated: ${ }^{*}, \mathrm{p}<0.05 ;{ }^{* *}, \mathrm{p}<0.01$. 
Table 3. Kidney pathology

\begin{tabular}{cccc}
\hline & Control mAb & CD20 mAb & CD19 mAb \\
\hline Tubulitis & $1.4 \pm 0.20$ & $0.88 \pm 0.01^{*}$ & $0.43 \pm 0.18^{* *, \#}$ \\
Interstitial inflammation & $2.2 \pm 0.17$ & $1.5 \pm 0.01^{* *}$ & $1.29 \pm 0.16^{* *}$ \\
Glomerulitis & $0.56 \pm 0.20$ & $0.38 \pm 0.18$ & $0.29 \pm 0.16$ \\
Arteritis & $0.89 \pm 0.35$ & $0.50 \pm 0.19$ & $0.29 \pm 0.16$ \\
Interstitial fibrosis & $0.67 \pm 0.19$ & $0.63 \pm 0.18$ & $0.86 \pm 0.13$ \\
Tubular atrophy & $0.56 \pm 0.20$ & $0.38 \pm 0.18$ & $0 \pm 00^{* *, \#}$ \\
Total score & $6.4 \pm 0.67$ & $4.4 \pm 0.80^{*}$ & $3.4 \pm 0.81^{* *}$ \\
\hline
\end{tabular}

Naïve huCD19Tg mice were treated with control, CD20, or CD19 mAb as in figure 7 before receiving a DBA/ 2 kidney allograft. After $21 \mathrm{~d}$, the mice were euthanized and a portion of the transplanted kidney was removed and placed in $10 \%$ buffered formalin. After formalin fixation, kidney tissue sections were stained with H\&E, PAS, and Masson's Trichrome. Slides were scored for renal injury based on the following scale: 0 , no abnormalities; 1 , mild; 2, moderate; and 3, severe. Values represent the mean ( \pm SEM) pathological score for the indicated parameter. Significant differences between sample means are indicated: ${ }^{*}, \mathrm{p} \leq 0.05$ vs. control mAb group; ${ }^{* *}, \mathrm{p} \leq 0.01$ vs. control mAb group; \#, $\mathrm{p}<0.05$ vs. CD20 mAb group. 


\subsubsection{CD19 mAb Treatment Depletes Pre-Existing Allograft-Specific $\lg G$}

Because CD19 mAb treatment depleted serum IgG levels in naïve mice and prevented the formation of allograft-specific $\operatorname{IgG}$ in mice receiving heart and kidney allografts, whether CD19 mAb could deplete pre-existing allograft-specific IgG levels was determined. huCD19Tg mice were immunized and boosted i.p. with $10^{7}$ DBA / 2 splenocytes at weeks 0 and 2. Three weeks after the boost (at the peak of the $\mathrm{Ab}$ response), the mice received control, CD20, or CD19 mAb. Serum DBA/2-specific IgG was assessed using indirect immunofluorescence staining with flow cytometry analysis. All mice in each of the three groups mounted similar allograft-specific IgG responses. At 10 weeks post-mAb treatments, CD19 mAb treatment reduced allograft-specific IgG levels by $59 \%$ compared to control mice ( $p=0.008)$, while CD20 mAb had no effect (Figure 18C). Thus, CD19 mAb treatment not only inhibited the induction of allograftspecific IgG in naïve mice, but reduced allograft-specific IgG levels in allo-sensitized mice. 


\subsection{Discussion}

B cell depletion by highly effective CD20 and CD19 mAbs in side-by-side comparisons confirmed that CD19 mAb depletes a broader spectrum of B cells in huCD19Tg mice than CD20 mAb due to CD19 being expressed both early and late during B cell development (Figure 13, Figure 15). As a consequence, chronic kidney allograft rejection was significantly inhibited by CD19, but not CD20 mAb treatment (Figure 18). Furthermore, CD19 mAb treatment inhibited the production of allograftspecific IgG during acute cardiac and chronic renal allograft rejection and reduced serum IgG and pre-existing levels of serum allograft-specific Ab, while CD20 mAb did not (Figure 16, Figure 17, Figure 18). These differences are explained by the finding that CD20 mAb depleted mature B cells, while CD19 mAb depleted both mature B cells and a significant fraction of plasmablasts and Ab-secreting plasma cells (Figure 13, Figure 14, Figure 15). Thereby, it is likely that allograft-specific Ab production in combination with B cell Ag presentation contributes to chronic renal allograft rejection. Thus, CD19 $\mathrm{mAb}$ may represent a new treatment for sensitized transplant recipients with preexisting allograft-specific $\mathrm{Abs}$, in addition to transplant patients undergoing $\mathrm{Ab}-$ mediated acute or chronic rejection, because it depletes a significant portion of the plasmablast and Ab-secreting B cell pool in addition to mature B cells.

While B cells contributed to chronic renal allograft rejection, B cell depletion accelerated skin graft rejection. Specifically, skin grafts expressing a single foreign $\mathrm{Ag}$ were rejected significantly faster following mature B cell depletion by CD20 mAb (Figure 17C). The proliferation of alloAg-specific $\mathrm{CD} 4^{+} \mathrm{T}$ cells in grafted mice was also significantly enhanced by B cell depletion (Figure 17D-E). Since CD20 mAb treatment only depletes B cells (27), these results demonstrate that some B cells normally function to inhibit allograft destruction. Most likely, CD20 mAb depletion of regulatory IL-10- 
competent B10 cells $(67,116)$ explains the hastened graft rejection and augmented alloAg-specific $\mathrm{CD}^{+} \mathrm{T}$ cell response observed in this allograft model. Similarly, B10 cell depletion by CD20 mAb treatment can exacerbate contact hypersensitivity responses and the onset and severity of $\operatorname{EAE}(58,68)$. Enhanced skin graft rejection in the absence of B cells has also been suggested previously; BALB / B skin may be rejected faster in $\mu \mathrm{MT}$ mice than in wild type C57BL / 6 mice (22), and act-mOVA skin grafts were rejected faster in four $\mu \mathrm{MT}$ mice (110). In a previous study, B cell depletion using a different CD20 mAb before grafting skin with a single MHC difference did not affect rejection, although all skin grafts were acutely rejected within $10 \mathrm{~d}$ in that model (166). Similarly, B cell depletion did not alter the acute rejection of MHC-mismatched skin grafts in the current study (Figure 17A). Thereby, since B cells do not act as APCs during skin graft rejection (153), the negative regulatory activities of B cells are likely to be most easily observed when alloAg concentrations are low and the Ab contributions of B cells to pathology are reduced. Thus, B cells can negatively regulate allograft rejection depending on the nature of the allograft and the intensity of the rejection response.

B cell contributions to acute cardiac rejection, chronic renal rejection, and skin graft rejection also depended on the nature of the allograft and the intensity of the rejection response. B cell depletion did not affect acute cardiac allograft survival (Figure 16) or alter the rejection of fully MHC disparate BALB / C skin grafted onto C57BL / 6 mice (Figure 17A). Similarly, mature B cell depletion by CD20 mAb did not affect chronic renal rejection (Figure 18). These findings are consistent with studies where $\mu \mathrm{MT}$ and wild type C57BL / 6 mice rejected BALB / C hearts (150), C3H hearts (151), or C3H aortic segments (152) similarly. Xenotransplants are also rejected normally in Xid mice (167), and BALB / C skin is rejected normally in agammaglobulinemic B10.BR mice treated chronically with anti-IgM serum to deplete B cells (168). Reduced allograftspecific Ab levels following B cell depletion did not appear to affect skin graft (Figure 
17A-B) or cardiac allograft (Figure 16) rejection, even though the addition of exogenous allograft-specific $\mathrm{Ab}$ is capable of inducing skin graft destruction $(169,170)$. Further, allograft-specific Abs can cause pathological lesions in mouse cardiac allografts (171) and allograft rejection in immunosuppressed mice $(150,154,155)$. B cells can also function as APCs during acute rejection (153). However, when Ag is present at high levels in B cell-depleted mice, DCs and/or macrophages appear sufficient for Ag presentation (27). Thereby, B cell depletion may only prolong allograft survival when carried out in conjunction with the suppression of T cell responses $(143,172)$ since vascularized grafts can represent an enormous allo-Ag pool that drives $\mathrm{T}$ cell activation. Consistent with this, CD20 mAb pretreatment abrogates cellular and humoral immune responses to model Ags and in autoimmune disease where the Ag doses are far lower than found in fully vascularized organ allografts $(2,27)$. Thus, mature B cell depletion may optimally prolong graft survival only when the alloAg load is reduced.

The current CD20 mAb results in mouse allograft models mimic what has been observed in the clinic when treating allograft rejection with the CD20 mAb Rituximab. Because Rituximab treatment has poor and variable effects on preformed allograftspecific Ab levels in patients (173), it is often combined with plasmapheresis, intravenous immunoglobulin (IVIG), splenectomy, and / or other immunosuppressive drugs to desensitize patients before grafting (174) or to prevent Ab-mediated rejection (175). For example, using a combination of Rituximab and IVIG for desensitization, $80 \%$ of patients could be transplanted, with $94 \%$ of grafts surviving at $1 \mathrm{yr}$ (176). Combining plasmapheresis, low dose IVIG, and Rituximab treatment has also successfully decreased existing allograft-specific $\mathrm{Ab}$ titers in some patients (177). In eight patients undergoing cardiac transplant rejection, humoral rejection was reversed when Rituximab was used as a first-line therapy in association with cyclosporine, prednisone, and mycophenolate (178). In another study, Rituximab, in combination with steroids, 
plasmapheresis, and/or anti-thymocyte globulin successfully treated biopsy-confirmed kidney rejection (179). Although the specific contribution of CD20 mAb treatment is clouded in these patients by the presence of other immunosuppressive therapies, the current comparative results obtained using CD19 mAb in side-by-side models of rejection argue that CD19 mAb may be even more effective than CD20 mAb for desensitization and the treatment of acute and chronic Ab-mediated rejection.

This study demonstrates that B cells can either positively or negatively regulate graft rejection depending on the nature of the allograft and the intensity of the rejection response. Inhibitory roles for regulatory B cells (B10 cells) during organ rejection may be commonly obscured by the strength of immune responses against mismatched tissue and organ grafts. Nonetheless, the potential for human B cell negative regulatory function during allograft rejection is suggested by a recent suspended clinical trial where patients receiving Rituximab suffered acute cellular rejection of kidney grafts at a greater rate (180). These and previous studies also indicate that mature B cell depletion alone with CD20 mAb will not be sufficient to prevent or treat solid organ allograft rejection due to the dominant and overlapping functions of $\mathrm{T}$ cells and other effector cells. Thereby, CD19 mAb may offer a new approach for depleting both B cells and some plasma cells to impair Ag presentation, reduce preexisting allograft-specific Ab levels, and inhibit the generation of new allograft-specific Abs. Thus, the current results emphasize that B cells contribute in multiple ways to rejection, and demonstrate the balance that exists between the positive and negative regulatory functions of B cells during allograft rejection. 


\title{
5. Maintenance of Long-Lived Plasma Cells and Serological Memory Despite Mature and Memory B Cell Depletion During CD20 Immunotherapy In Mice
}

\author{
The following text was slightly modified from its original manuscript, \\ “Maintenance of Long-Lived Plasma Cells and Serological Memory Despite Mature and \\ Memory B Cell Depletion During CD20 Immunotherapy In Mice," published in 2008 in \\ The Journal of Immunology.
}

\subsection{Introduction}

The B1, MZ, and GC B cell subsets contribute to the circulating natural Ab pool, T cell-independent $(\mathrm{TI})^{4} \mathrm{IgM} \mathrm{Ab}$ responses, and adaptive immunity (181-184) by terminal differentiation into plasma cells, the effector cells of humoral memory and immunity $(86,94)$. Ag-activation of mature B cells leads initially to the development of GCs, the transient generation of plasmablasts that secrete $\mathrm{Ab}$ while still dividing, and short-lived plasma cells that secrete Ag-specific germline-encoded antibodies (6). Extrafollicular plasma cells with half-lives of 3-5 d appear first in the T cell zones (7), and then migrate to the red pulp of the spleen (185). In GCs, Ag-specific B cells proliferate, diversify their receptors, and give rise to pools of long-lived memory B cells (8). Memory $\mathrm{B}$ cells generated during the second week of primary Ab responses express mutated Ag receptors with enhanced affinities for Ag (9-11). The cellular precursors contributing to memory $\mathrm{Ab}$ responses are classically defined as surface $\operatorname{IgM}^{+} \operatorname{Ig} \mathrm{D}^{-} \mathrm{B} 220^{+}$or $\operatorname{IgM}^{-} \operatorname{IgD} \mathrm{D}^{-} \mathrm{B} 220^{+}$ Ag-binding B cells that do not express Syndecan-1 (CD138) (10, 186-188). Memory B cells persist in substantial numbers for at least 8 weeks after Ag challenge, rapidly expand during secondary responses, and terminally differentiate into plasma cells (12). Thereby, post-GC memory $\mathrm{B}$ cells give rise to $\mathrm{Ab}$ isotype-switched plasmablasts and then long-lived plasma cells once outside of GCs. 
Persistent Ag-specific Ab titers are thought to derive from long-lived plasma cells $(12,86)$. Ab-secreting plasma cells generated in the spleen migrate to the BM where they may persist for the life of the animal without the need for self-replenishment or turnover $(6,93,94,96-98)$. In fact, BM cell transfers confer long-lived Ab production, presumably due to the transfer of long-lived plasma cells (97). Nonetheless, Radbruch and colleagues state in a recent review, "The biology of plasma cells... remains enigmatic and is of considerable debate. Of particular interest is whether plasma cells are long-lived and form a pool of memory plasma cells that is separate from the pool of memory B cells" (86). In one model for the maintenance of serum Ab levels, primary and secondary immune responses generate a pool of long-lived plasma cells that occupy essential survival niches within the BM where they survive indefinitely, resulting in long-lived serum Ab levels $(7,93-95)$. In a second model, persisting Ag drives the memory B cell pool to chronically generate plasmablasts and short-lived plasma cells that result in long-lived Ab production (90). In a third model, memory cells themselves (91, 92), or pre-plasma cell precursors (189) continuously give rise to plasma cells in an Agindependent manner as a means of maintaining serum $\mathrm{Ab}$ levels for extended time periods. In this third model, Ag-independent cytokines or TLR signals continuously induce a subset of memory B cells to differentiate into plasma cells with defined halflives. These three models are not mutually exclusive, but all hinge on understanding whether long-lived plasma cells are distinct from the memory B cell pool or other precursor populations.

Mature $\mathrm{CD} 20^{+} \mathrm{B}$ cell depletion is proving to be effective in treating B cell malignancies and some autoimmune diseases $(190,191)$. CD20 is a B cell-specific molecule that is first expressed on the cell surface during the pre-B to immature B cell transition, but is presumed to be lost upon plasma cell differentiation $(104,161)$. However, it has been difficult to determine the precise effects of B cell depletion on 
human B cell subsets and humoral immunity since most CD20 mAb therapy patients are also given immunosuppressive drugs (190). To assess the effects of CD20 ${ }^{+} \mathrm{B}$ cell depletion on GC B cells, memory B cells, plasmablasts, Ab-forming plasma cells, existing $\mathrm{Ab}$ levels, and primary and memory $\mathrm{Ab}$ responses in mice, $\mathrm{B}$ cells were depleted using CD20 mAb $(39,43,71,72,104,133,192)$. While mature and memory B cells were $\mathrm{CD}_{20}{ }^{+}$and were effectively depleted by CD20 mAb treatment in vivo, CD20 $0^{+}$B cell depletion did not deplete long-lived plasma cell numbers or negatively affect long-lived serum Ab levels. Thus, the BM plasma cell pool does not require ongoing contributions from the memory B cell pool for its maintenance.

\subsection{Results}

\subsubsection{Serum Ig Levels Following CD20 ${ }^{+}$B Cell Depletion}

The influence of B cell depletion on serum Ig levels was assessed in littermates after CD20 (MB20-11) or control mAb treatments (250 $\mu \mathrm{g})$. At this mAb dose, circulating, spleen, and LN B cell numbers were reduced by $>95 \%$ by day 2 , and began to recover on day 57 (data not shown, ref. 39, 71, 133). Despite effective B cell depletion, serum IgM, IgG, and IgA levels were similar for both CD20 and control mAbtreated littermates for up to $58 \mathrm{~d}$ following mAb treatment (Figure 20A). Thus, CD20 ${ }^{+} \mathrm{B}$ cell depletion did not significantly reduce basal serum Ig levels.

Since peritoneal B cells are not efficiently depleted by CD20 mAb treatment (39, 71), their contribution to basal serum Ig levels was assessed. Thioglycollate given i.p. $1 \mathrm{~d}$ prior to CD20 mAb treatment induces significant peritoneal B cell depletion within $7 \mathrm{~d}$ (39). B cell depletion prevented the increase in serum Ig levels that follows thioglycollateinduced inflammation. In addition, thioglycollate plus CD20 mAb treatment reduced serum IgM $\sim 40 \%$ from initial levels ( $\mathrm{p}<0.05$ ), while serum IgG1, IgG2b, IgG3, or IgA levels did not fall below the normal range (Figure 20B). To assess whether prolonged CD20 
$\mathrm{mAb}$ treatment might also affect serum Ig levels, 3-mo-old human CD19 transgenic (huCD19Tg) mice were treated bi-weekly with a CD20 mAb dose (5 $\mu \mathrm{g})$ that effectively depletes circulating and peripheral B cells (133). Serum Ig levels increase at a faster rate than normal in autoimmune-prone CD19-transgenic mice, as described (103).

Continuous $\mathrm{CD}_{20}{ }^{+}$B cell depletion did not significantly affect basal IgM or IgG levels, but did prevent the development of hypergammaglobulinemia (Figure 20C). Thus, $\mathrm{CD} 20^{+} \mathrm{B}$ cell depletion significantly attenuated increases in serum Ig, but had minimal effects on basal serum Ig levels. 

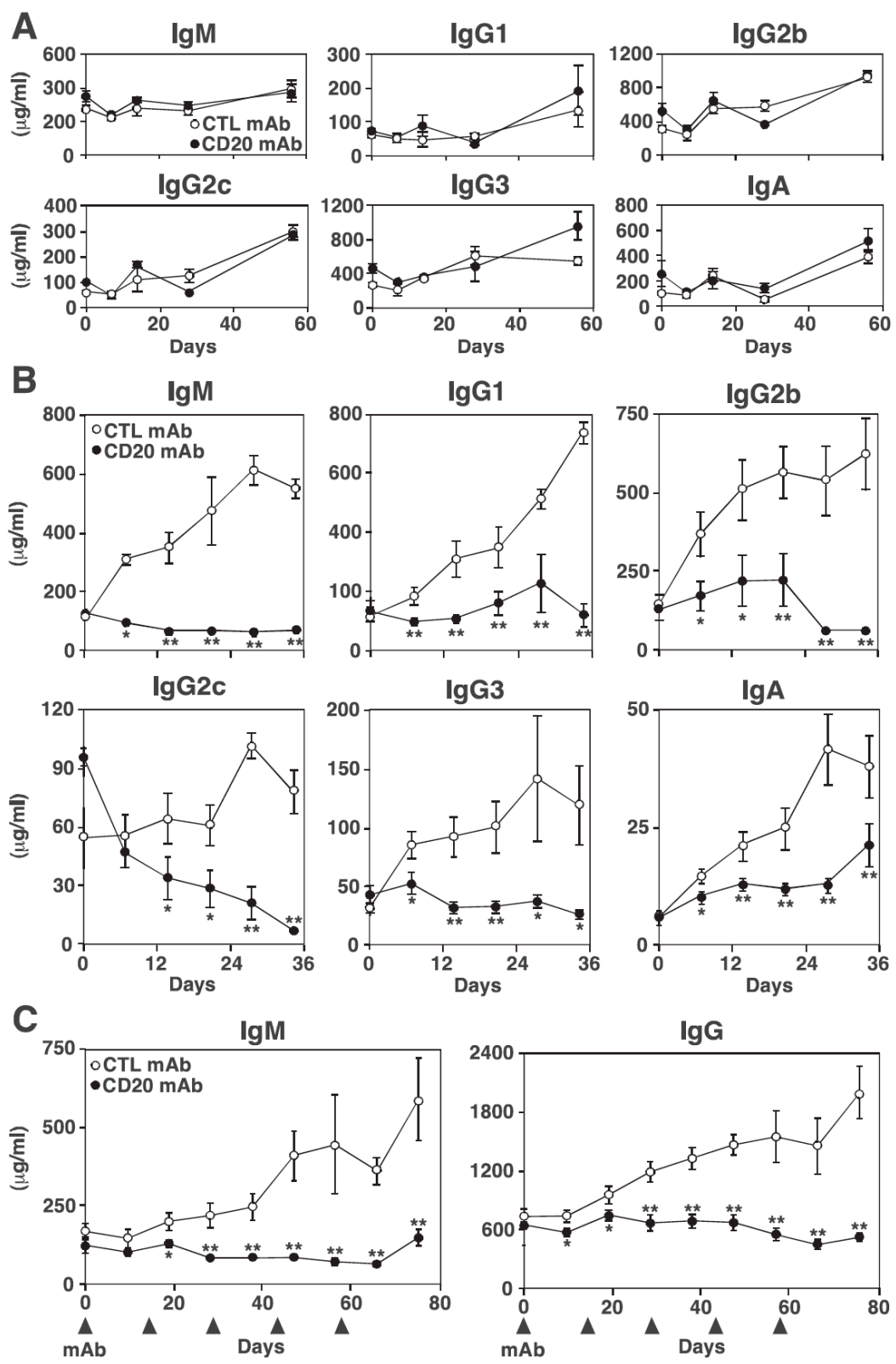

Figure 20: Humoral immunity following $\mathrm{CD}_{20}{ }^{+}$B cell depletion. (A) Serum Ig levels in CD20 mAb-treated mice. Two-mo old littermate pairs of mice $(n \geq 3)$ were treated with MB20-11 or control (CTL) $\mathrm{mAb}$ on day 0 . (B) Effect of $\mathrm{CD} 20^{+} \mathrm{B}$ cell depletion on inflammation-induced immune responses. Two-mo old littermate pairs of mice were given thioglycollate solution followed by MB20-11 ( $\mathrm{n}=10)$ or control $(\mathrm{n}=10) \mathrm{mAb}$ treatment one d later on day 0 . (C) Effect of continuous B cell depletion on serum IgM and IgG levels in huCD19Tg mice. Mice were treated bi-weekly with $5 \mu \mathrm{g}$ of CD20 $(n=5)$ or control $(n=5)$ mAb every 2 weeks for 2 mo. A-C) Values represent mean relative Ig levels $( \pm$ SEM) in individual serum samples obtained on the days indicated as determined by isotype-specific ELISA. Differences between CD20 and control mAbtreated mice were significant, $* \mathrm{p}<0.05 ; * * \mathrm{p}<0.01$. 


\subsubsection{Ab Responses Following CD20 ${ }^{+}$B Cell Depletion}

$\mathrm{Ab}$ responses to the TD Ag DNP-KLH were assessed in mice treated with CD20 mAb $7 \mathrm{~d}$ before immunization. CD20 and control mAb-treated littermates generated similar primary IgM Ab responses $7 \mathrm{~d}$ after DNP-KLH immunization (Figure 21A). However, IgM Ab responses in CD20 mAb-treated mice decayed rapidly between 2-4 weeks following immunization relative to control mAb-treated littermates. By contrast, DNP-specific IgG responses were 20-fold lower in CD20 mAb-treated mice compared with control mAb-treated littermates on day 28. All mice were re-treated with CD20 or control mAb on day 21, and were re-challenged with DNP-KLH on day 28. Secondary IgM and IgG Ab responses were impaired (15- and 40-fold lower, respectively) in CD20 mAb-treated mice by day 35, while significant secondary Ab responses were observed in control mAb-treated littermates (Figure 21A). When mice were treated with CD20 mAb plus thioglycollate, primary IgM responses to DNP were significantly decreased, with 6fold lower IgM Ab titers on day 7 and 77-fold lower IgG titers on day 28 (Figure 21B). Secondary IgM and IgG titers were 30- and 36-fold lower on day 35, respectively. Thus, initial TD IgM Ab responses progressed normally after B cell depletion due predominantly to peritoneal B cells, while class switched and secondary Ab responses were severely inhibited.

The influence of B cell depletion on TI humoral immune responses was assessed in littermates treated with CD20 or control mAb $7 \mathrm{~d}$ before TNP-LPS (TI-1 Ag) or DNPFicoll (TI-2 Ag) immunizations. Following TNP-LPS immunizations, hapten-specific IgM levels were similar in both CD20 and control mAb-treated littermates, while TNPspecific IgG titers were significantly lower in CD20 mAb-treated mice (Figure 21C). With thioglycollate and CD20 mAb treatment before TNP-LPS immunization, both TNPspecific $\operatorname{IgM}$ and $\operatorname{IgG}$ levels were significantly decreased (Figure 21D). Hapten-specific 
IgM and IgG responses to DNP-Ficoll were also significantly reduced in CD20 mAbtreated mice compared with control mAb-treated littermates (Figure 21E). Thus, CD20 B cell depletion reduced TI-1 IgG and TI-2 IgM and IgG responses, while peritoneal B cell depletion was necessary to abrogate TI-1 IgM responses. 


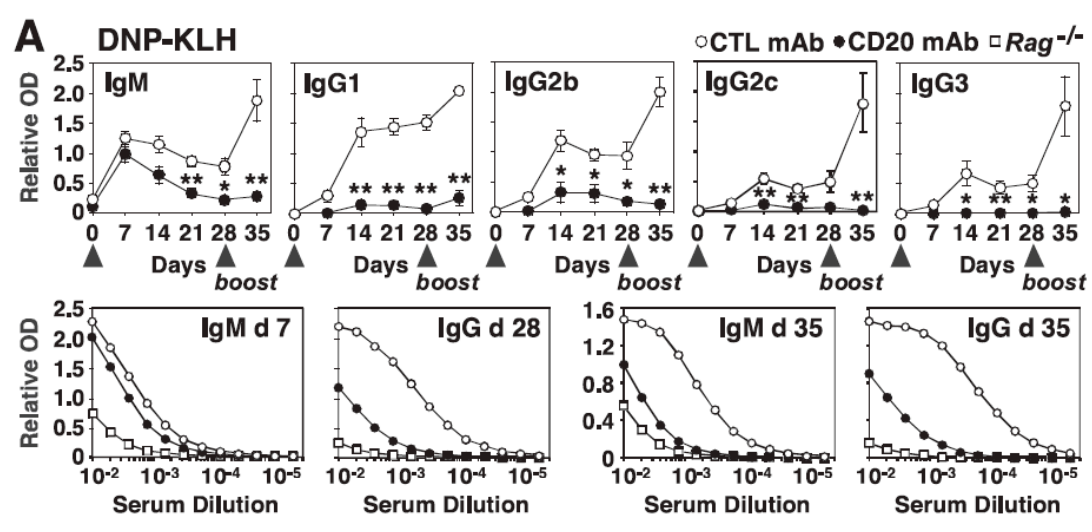

B DNP-KLH (Thioglycollate) OCTL mAb •CD20 mAb $\square$ Rag $/-$
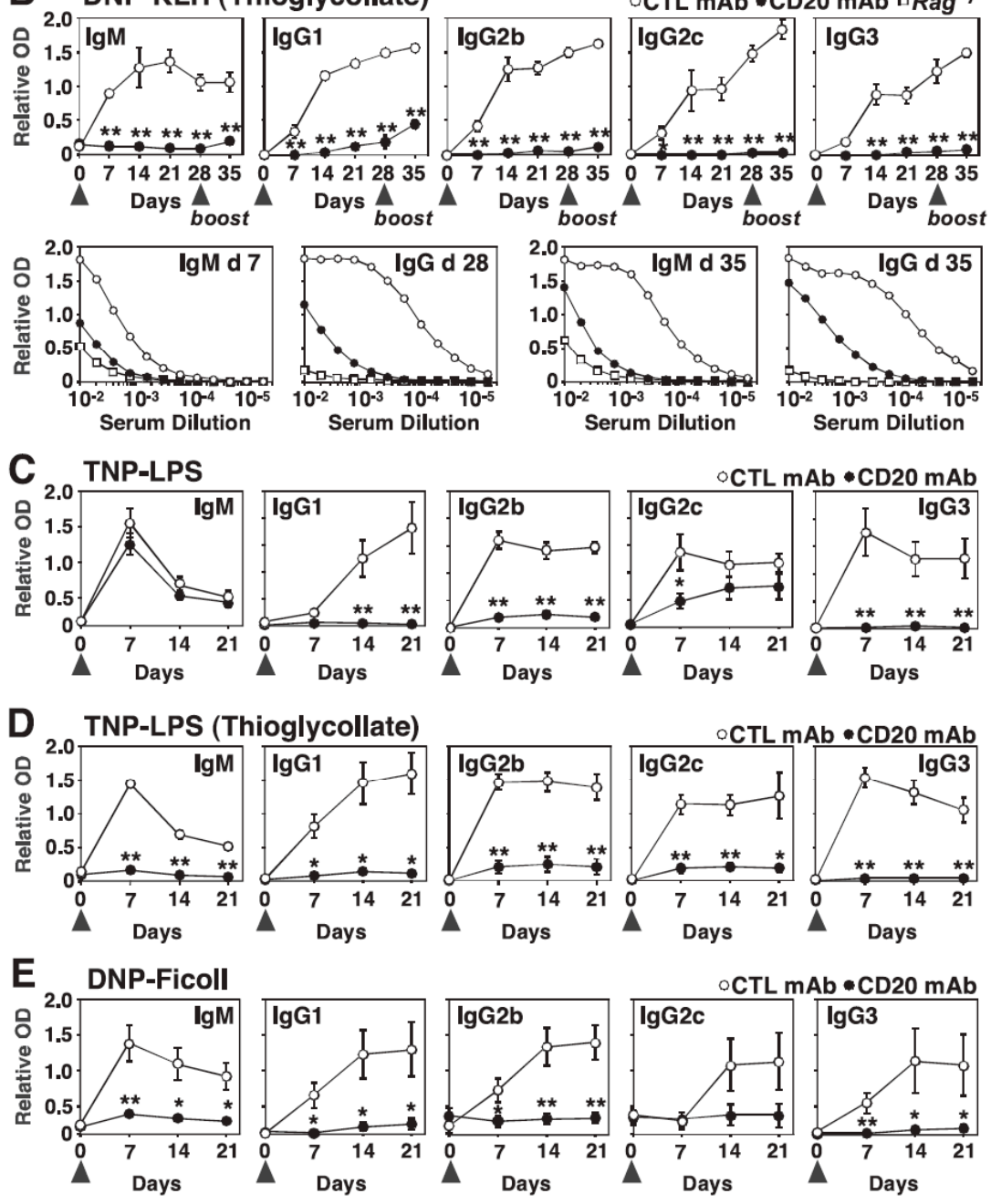

Figure 21: $\mathrm{TD}$ and $\mathrm{TI} A \mathrm{Ab}$ responses in $\mathrm{CD}^{+} \mathrm{B}^{+} \mathrm{B}$ cell depleted mice. (A) Effect of $\mathrm{B}$ cell depletion on DNP-KLH Ab responses. (B) Effect of thioglycollate treatment and B cell depletion on DNP-KLH Ab responses. A-B) Mice were retreated with mAb on day 21 and challenged with DNP-KLH on day 28. Lower panels indicate the relative titers of $\mathrm{DNP}$-specific IgM and IgG $\mathrm{Ab}$ in pooled sera from immunized mice with background 
Figure 21 (Continued) measured using $\mathrm{Rag}^{-/-}$mouse serum. (C-D) Effect of B cell depletion and thioglycollate treatment on TNP-LPS Ab responses. (E) Effect of B cell depletion on DNP-Ficoll Ab responses. A-E) Littermate pairs of mice were treated with MB20-11 or control $\mathrm{mAb}$ on day -7 with serum harvested before immunization (arrow) on days 0 . Hapten-specific Ab levels in individual serum samples harvested on the indicated days were measured by ELISA. Values represent mean $( \pm S E M)$ relative OD units obtained using individual sera from 5 mice of each group. Differences between CD20 or control mAb-treated mice were significant, ${ }^{*} \mathrm{p}<0.05 ;{ }^{* *} \mathrm{p}<0.01$. 


\subsubsection{GC B Cells are Depleted by CD20 mAb Treatment}

The effect of CD20 mAb treatment on GC B cells was assessed by immunofluorescence staining. Most B220 $\mathrm{GL}-7^{\text {high }}$ GC B cells expressed CD20 at levels 7-fold higher than those found on B220+GL-7 follicular B cells (Figure 22A). However, a small population (1-2\%) of GC B cells expressed little to no CD20. Since GC cells are maximally established by day 8 following immunizations with NP-CGG (185), mice were treated with CD20 or isotype control $\mathrm{mAb}$ on day 7 after immunization. On day $14, \mathrm{~B} 220^{+} \mathrm{GL}-7^{\text {high }}$ GC cell numbers were decreased by $99 \%$ in naïve mice following CD20 mAb treatment, and by 75\% in immunized mice (Figure 22B,C). The majority of $\mathrm{B} 220^{+} \mathrm{B}$ cells remaining within the spleens of immunized mice following $7 \mathrm{~d}$ of CD20 $\mathrm{mAb}$ treatment represented recent pre- $\mathrm{B}$ and immature $\mathrm{B}$ cell emigrants from the $\mathrm{BM}$ that had not begun to express CD20 at significant densities, as described (39). By contrast, mature BM $\left(B 220^{\text {high }} \operatorname{IgM}^{+}\right)$, blood $\left(B 220^{+}\right)$, mature spleen $\left(B 220^{+} \mathrm{CD} 24^{+} \mathrm{CD} 21^{+}\right)$, spleen T1 $\left(\mathrm{B} 220^{+} \mathrm{CD} 24^{\text {high }} \mathrm{CD} 21^{-}\right)$, spleen T2 $\left(\mathrm{B} 220^{+} \mathrm{CD} 24^{\text {high }} \mathrm{CD} 21^{+}\right)$, and $\mathrm{MZ}$ $\left(\mathrm{B} 220^{+} \mathrm{CD} 1 \mathrm{~d}^{\text {high }} \mathrm{CD} 21^{\text {high }}\right) \mathrm{B}$ cells were depleted by 92-99\% following CD20 mAb treatment in naive mice and mice immunized with NP-CGG or DNP-KLH (Figure 22C, Table 4). Histological examination of spleen sections revealed dramatically reduced follicular and GC B cell staining $7 \mathrm{~d}$ after CD20 mAb-treatment (Figure 22D). Therefore, immunization does not affect the overall efficiency of B cell depletion, and most GC B cells expressed CD20 at high levels, thus making them sensitive to CD20 mAb-mediated depletion. 

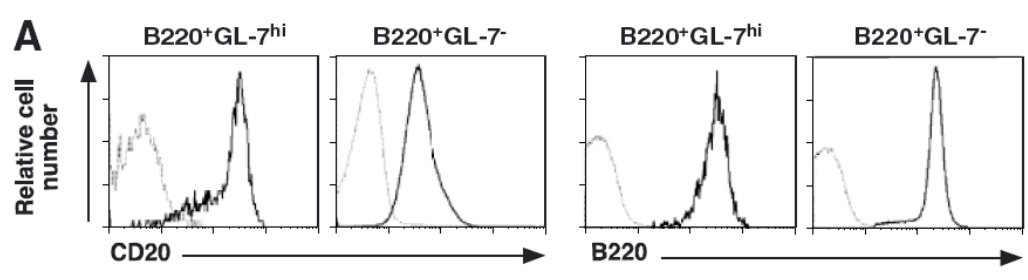

B
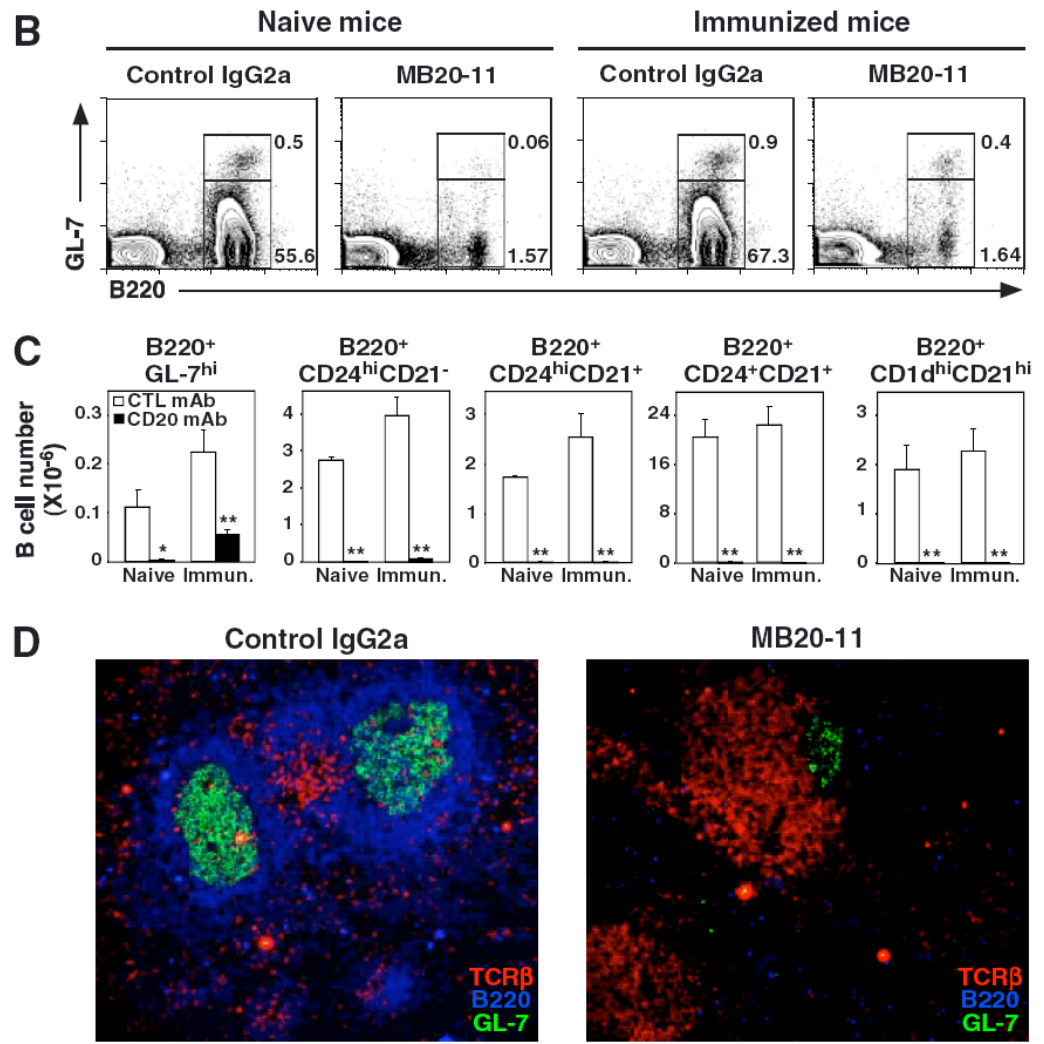

Figure 22: GCs in B cell depleted mice. (A) Representative CD20 expression by B cells with GC (B220 GL-7 $\left.{ }^{\text {high }}\right)$ and follicular (B220 ${ }^{+}$GL-7 $)$phenotypes assessed by three-color immunofluorescence staining with flow cytometry analysis. Mice were immunized with $\mathrm{NP}_{18}$-CGG $8 \mathrm{~d}$ before splenocytes were isolated and stained using MB20-11 or B220 $\mathrm{mAbs}$ (heavy line) or isotype-matched control (light line) $\mathrm{mAb}$ with fluorochromeconjugated IgG2a-specific secondary Ab. (B) Representative GC B cell depletion. Mice were untreated or immunized with $\mathrm{NP}_{18}$-CGG $7 \mathrm{~d}$ before being given MB20-11 or control $\mathrm{mAb}$ for $7 \mathrm{~d}$. Numbers indicate the relative percentages of $\mathrm{B} 220^{+} \mathrm{GL}-7^{\text {high }}$ or $\mathrm{B} 220^{+} \mathrm{GL}-7^{-}$ lymphocytes within the indicated gates. (C) Spleen B cell subsets. Bar graphs indicate mean $( \pm$ SEM) numbers of each $B$ cell subset in naïve and immunized littermates $7 \mathrm{~d}$ following MB20-11 or isotype control $\mathrm{mAb}$ treatment as in (B). Significant differences between sample means are indicated; ${ }^{*} \mathrm{p}<0.05,{ }^{* *} \mathrm{p}<0.01$. (D) Representative spleen GC depletion. Mice were immunized with NP-CGG and injected with control or CD20 mAb $7 \mathrm{~d}$ later. Seven $\mathrm{d}$ after $\mathrm{mAb}$ injection, spleens were harvested, sectioned, and stained for TCR $\beta$ chain, B220, and the GL-7 Ag. A-D) All results represent $\geq 3$ independent experiments. 
Table 4. Immunization effects on CD20 mAb-induced B cell depletion

\begin{tabular}{|c|c|c|c|c|c|}
\hline \multirow[b]{2}{*}{ Tissue } & \multirow[b]{2}{*}{ B Subset $^{b}$} & \multicolumn{2}{|c|}{ Naïve mice } & \multicolumn{2}{|c|}{ Immunized mice } \\
\hline & & Control & MB20-11 & Control & MB20-11 \\
\hline \multirow[t]{3}{*}{ BM: } & pro/pre & $2.7 \pm 0.3$ & $3.2 \pm 0.5$ & $1.5 \pm 0.1$ & $1.7 \pm 0.1$ \\
\hline & immature & $1.9 \pm 0.2$ & $1.6 \pm 0.2$ & $0.32 \pm 0.04$ & $0.19 \pm 0.02^{*}$ \\
\hline & mature & $2.6 \pm 0.3$ & $0.06 \pm$ & $0.20 \pm 0.04$ & $0.01 \pm 0.01^{* *}$ \\
\hline Blood: & $\mathrm{B} 220^{+}$ & $4.6 \pm 0.4$ & $0.08 \pm$ & $9.7 \pm 0.3$ & $0.05 \pm 0.01^{* *}$ \\
\hline \multirow[t]{4}{*}{ Spleen: } & Mature & $32 \pm 3$ & $0.7 \pm 0.1^{* *}$ & $22 \pm 3$ & $0.75 \pm 0.2^{* *}$ \\
\hline & $\mathrm{T} 1$ & $4.9 \pm 1.0$ & $0.7 \pm 0.1^{* *}$ & $4.6 \pm 0.9$ & $1.3 \pm 0.2^{* *}$ \\
\hline & $\mathrm{T} 2$ & $2.5 \pm 0.7$ & $0.01 \pm$ & $5.9 \pm 1.2$ & $0.14 \pm 0.04^{* *}$ \\
\hline & MZ & $1.6 \pm 0.5$ & $0.01 \pm$ & $3.5 \pm 0.3$ & $0.02 \pm 0.01^{* *}$ \\
\hline Peripheral LN: & $\mathrm{B} 220^{+}$ & $1.1 \pm 0.1$ & $0.09 \pm$ & $2.2 \pm 0.3$ & $0.16 \pm 0.07^{* *}$ \\
\hline
\end{tabular}

Mice were either unimmunized or injected with DNP-KLH on day 0 . On day 28, the immunized mice were boosted with DNP-KLH. Naive mice received control or CD20 $\mathrm{mAb}$ on day 0 , with tissues harvested on day 7 . Immunized mice were injected with control or CD20 mAb on day 35, with tissues harvested on day 42 . B cell subsets were: $\mathrm{BM}$ pro/pre-B $\left(\mathrm{B} 220^{\text {low }} \operatorname{IgM}^{-}\right)$, immature B $\left(B 220^{\text {low }} \operatorname{IgM}^{+}\right)$; and mature $B\left(B_{2} 20^{\text {high }} \operatorname{IgM}^{+}\right)$; spleen mature $\left(\mathrm{B} 220^{+} \mathrm{CD} 24^{+} \mathrm{CD} 21^{+}\right), \mathrm{T} 1\left(\mathrm{~B} 220^{+} \mathrm{CD} 24^{\text {high }} \mathrm{CD} 21^{-}\right), \mathrm{T} 2$ $\left(\mathrm{B} 220^{+} \mathrm{CD} 24\right.$ highCD21 $\left.{ }^{+}\right)$, and marginal zone $\left(\mathrm{MZ}, \mathrm{B} 220^{+} \mathrm{CD} 21^{\text {high }} \mathrm{CD} 1 \mathrm{~d}^{+}\right)$; LN, LN. Values $\left( \pm\right.$ SEM) indicate cell numbers $\left(\times 10^{-6}\right)$ present in mice $7 \mathrm{~d}$ after $\mathrm{mAb}$ treatment $(\mathrm{n} \geq 3)$ : blood shown as cells $\left(\times 10^{-5}\right) / \mathrm{ml}, \mathrm{LN}$ as pooled bilateral inguinal and axial $\mathrm{LN}$, and BM as both femurs pooled. Significant differences between means are indicated: *, $\mathrm{p}<0.05 ;{ }^{* *}, \mathrm{p}<0.01$. 


\subsubsection{Memory B Cells and Ab Responses Are Depleted by CD20 mAb}

The effect of $\mathrm{CD} 20^{+} \mathrm{B}$ cell depletion on memory Ab responses was assessed using DNP-KLH immunized mice that were then treated with CD20 or control mAb 21 d later. Despite effective B cell depletion for 57 d as described $(39,71)$, significant DNP-specific IgM and IgG Ab levels were maintained in CD20 and control mAb-treated mice for at least $77 \mathrm{~d}$ following primary immunizations (Figure 23A). The mice were then retreated with CD20 or control mAb on day 82 and re-challenged with DNP-KLH $7 \mathrm{~d}$ later. Secondary Ab responses were elicited in control mAb-treated mice, but IgM levels were 26-fold lower (day 96) and IgG titers were 6-fold lower (day 104) in CD20 mAbtreated mice (Figure 23B). To compare primary and secondary Ab responses, naïve mice were immunized with Ag in parallel with the re-challenge of their previously immunized littermates. These primary IgM responses were greater than secondary responses in previously immunized mice after CD20 mAb treatment, but IgG responses were relatively modest (Figure 23A,B). Thus, CD20 mAb treatment inhibited memory IgM and IgG B cell responses, but did not deplete preformed serum Ab.

Adoptive transfer experiments were carried out to verify the effect of CD20 mAb treatment on memory $\mathrm{Ab}$ responses and to exclude the induction of plasma cells from primary immune responses. NP-CGG-primed B220 ${ }^{+}$B cells or naive B cells were mixed with equal numbers of CGG-primed Thy $1.2^{+} \mathrm{T}$ cells, and transferred into $\mathrm{Rag} 1^{-1-}$ mice before treatment with CD20 or control mAbs and challenge with NP-CGG. NP-specific IgG1 Ab titers and avidities were determined by ELISA using low-valency $\mathrm{NP}_{5}$-BSA or high-valency $\mathrm{NP}_{25}$-BSA. NP-specific IgG1 antibodies $(400 \pm 50 \mu \mathrm{g} / \mathrm{ml})$ were easily observed in control mAb-treated $\mathrm{Rag}^{-/-}$mice receiving NP-primed B cells, while Ab levels were not measurable in CD20 mAb-treated mice (Figure 23C). Control mAbtreated $\operatorname{Rag}^{-/-}$mice given naïve B cells had low NP-specific IgG1 Ab levels. The NP- 
specific antibodies produced in control $\mathrm{mAb}$-treated $\mathrm{Rag} 1^{-/-}$mice receiving primed $\mathrm{B}$ cells were high avidity $\left(\mathrm{NP}_{5} / \mathrm{NP}_{25}=0.91\right)$, while $\mathrm{NP}_{5} / \mathrm{NP}_{25}$ ratios were lower $\left(\mathrm{NP}_{5} / \mathrm{NP}_{25}=0.13\right)$ in $\mathrm{Rag1}^{-/-}$mice given naive $\mathrm{B}$ cells (Figure 23D). The numbers of plasma cells and plasmablasts measured functionally as NP-specific IgG1 ASCs in the BM and spleens of control mAb-treated $\operatorname{Rag} 1^{-/-}$mice that received primed B cells were $13 \pm 4$ and $643 \pm 196$ (per $10^{5}$ cells), respectively. However, NP-specific ASCs were undetectable in CD20 mAb-treated mice (Figure 23E). $R a g 1^{-1-}$ mice given naïve B cells generated spleen ASCs at very low numbers, but none were detected in the BM. Thus, CD20 mAb treatment effectively depleted memory B cells and thereby eliminated secondary Ab responses. 

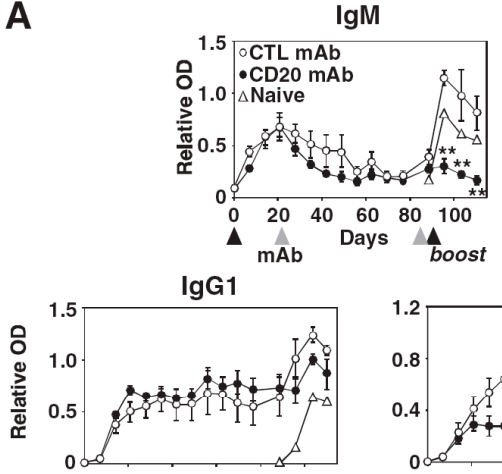

$\lg \mathbf{G} 2 \mathbf{b}$
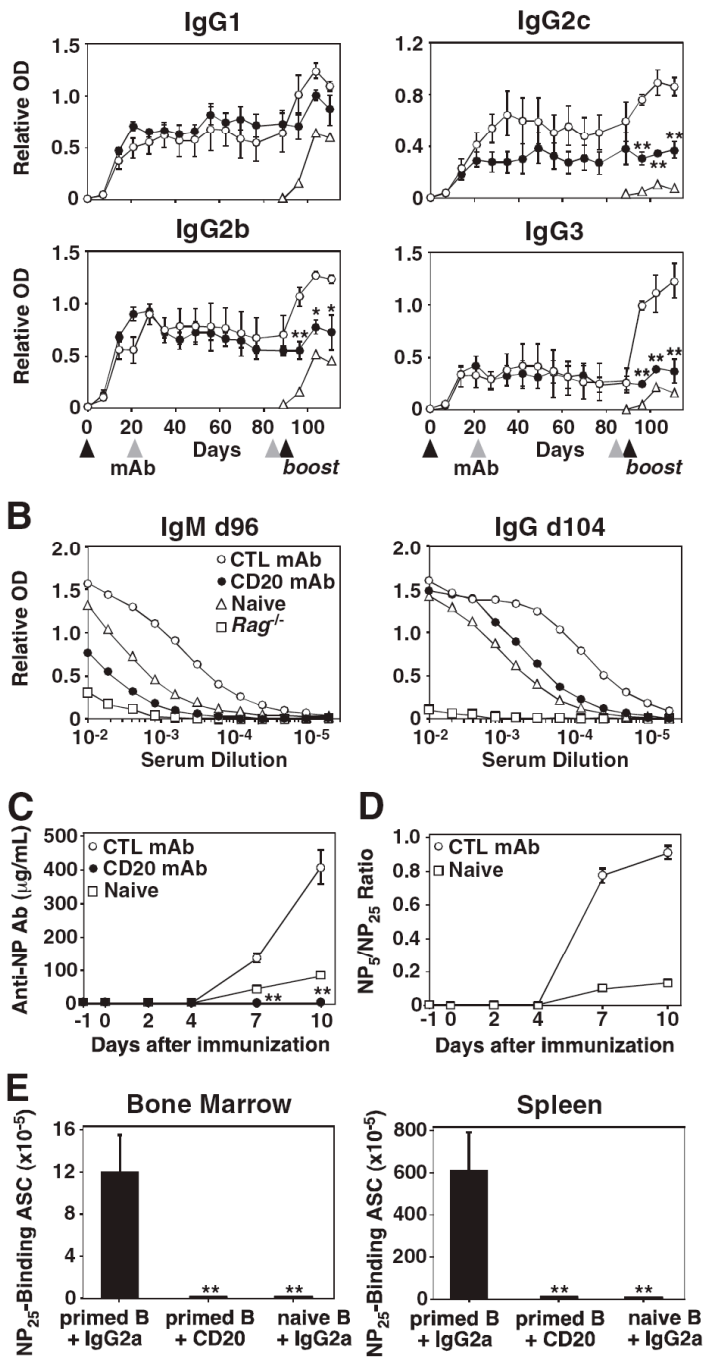

Figure 23: Long-lived Ag-specific Ab levels and memory $\mathrm{Ab}$ responses in $\mathrm{CD}_{20}{ }^{+} \mathrm{B}$ cell-depleted mice. (A) Ag-specific Ab levels after B cell depletion. Littermate pairs of mice were immunized (filled arrows) with DNP-KLH on day 0 and treated with MB20$11(n=5)$ or control $(n=5)$ mAb on days 21 and 82 (shaded arrows). All mice were rechallenged with DNP-KLH on day 89. Previously unimmunized littermates were also immunized with DNP-KLH on day 89 to quantify primary Ab responses. (B) Relative titers of DNP-specific IgM (day 96) and IgG (day 104) in pooled sera from immunized mice in (A) with background measured using Rag $1^{-1-}$ mouse serum. (C-D) Memory Ab responses after adoptive transfer. Purified splenic B220 ${ }^{+}$cells were isolated from NPCGG-primed mice (42 d after immunization) or from unimmunized littermates and 
Figure 23 (Continued) adoptively transferred into Rag ${ }^{-1-}$ mice (day -1 ) along with an equal number of splenic T cells isolated from CGG-primed mice. The mice were then immediately given MB20-11 or control mAb. All mice were boosted with NP-CGG on day 0 , with serum harvested on the indicated days. A-C) Hapten-specific Ab levels in individual serum samples were measured by isotype-specific ELISA. Values represent mean $( \pm S E M)$ relative OD units obtained using individual sera (unless indicated otherwise) from $\geq 4$ mice in each group. Significant differences between CD20 and control $\mathrm{mAb}$-treated mice are indicated; ${ }^{*} \mathrm{p}<0.05,{ }^{* *} \mathrm{p}<0.01$. (D) High-affinity NP-specific IgG1 levels in individual serum samples from $(C)$ were measured by ELISA using $\mathrm{NP}_{5}$-BSA and $\mathrm{NP}_{25}$-BSA to indicate the relative affinities of the $\mathrm{Ab}$ responses. Values represent mean $( \pm S E M)$ ratios of the titers for each serum sample. (E) BM and spleen ASC responses. Numbers of cells secreting $\mathrm{NP}_{25}$-specific IgG1 were determined by ELISPOT. Values indicate mean $( \pm$ SEM) ASC numbers from mice in $(C)$ on day 10 . Less than 0.1 ASC $/ 10^{5}$ cells were detected in the BM and spleens of mice with primed B cells treated with MB20-11 (primed B + CD20) and naive B cells treated with control mAb (naive B + IgG2a). Differences between mice with primed $B$ cells treated with control $\mathrm{mAb}$ (primed B + IgG2a), and primed B cells plus CD20 mAb or naive B cells plus IgG2a mAb were significant; ${ }^{* *} \mathrm{p}<0.01$. 


\subsubsection{CD20 Expression by Plasmablasts and Plasma Cells In Vitro and In Vivo}

Plasmablasts and plasma cells express CD138, a commonly-used marker for ASCs $(158,159)$. Plasmablasts may be induced in vitro by stimulating B cells with LPS to induce TLR-dependent plasma cell differentiation or with CD40 mAb in combination with IL-4 to mimic TD plasma cell differentiation (108). Therefore, B cells were labeled with CFSE, cultured with LPS or CD40 mAb plus IL-4, and divided into three subpopulations based on their relative CFSE intensities and CD138 expression (193). After LPS stimulation, proliferating plasmablasts $\left(\mathrm{CFSE}^{\mathrm{low}} \mathrm{CD} 138^{+}\right)$expressed CD20 at significant levels (Figure 24A). CFSE ${ }^{\text {low }} \mathrm{CD} 138^{-}$proliferating cells and $\mathrm{CFSE}^{\text {high }} \mathrm{CD}^{-}{ }^{-}$ non-proliferating cells also expressed CD20, but at levels $60 \%$ lower than plasmablasts. Similar results were obtained following B cell activation with CD40 mAb plus IL-4 stimulation (data not shown). Thus, plasmablasts generated in vitro expressed cell surface CD20.

While CD20 may be expressed on in vitro-derived ASCs, CD20 is downregulated in human B cells differentiating to plasma cells (161). To determine whether CD20 is downregulated in murine ASCs in vivo, CD20 expression was assessed by immunofluorescence staining of spleen and BM from naïve mice. B220 ${ }^{\text {low }}$ CD $138^{\text {high }}$ cells in the BM expressed CD20, but at densities $80 \%$ and $75 \%$ lower than splenic and BM $\mathrm{B} 220^{+} \mathrm{CD} 138^{-} \mathrm{B}$ cells, respectively (Figure 24B). In fact, when spleen and BM single-cell suspensions from naive mice were sorted with magnetic beads into $\mathrm{CD}_{2} 0^{+}$and $\mathrm{CD} 20^{-}$ populations, the CD20+ $0^{+}$populations of cells contained the majority of ASCs (Figure 24C). Thus, ASCs expressed CD20 at low, but detectable levels in vivo. 


\subsubsection{ASCs Resist CD20 mAb-Mediated Depletion In Vivo}

Because CD20 expression is drastically reduced on ASCs in vivo, the effect of CD20 mAb on ASC numbers in naïve mice was determined. Using CD138 staining with in vivo BrdU labeling techniques, in vivo-generated short-lived plasmablasts $\left(\mathrm{CD} 138^{\text {high }} \mathrm{BrdU}^{+}\right)$and long-lived plasma cells $\left(\mathrm{CD} 138^{\text {high }} \mathrm{BrdU}^{-}\right)$have been phenotypically recognized (160). Therefore, mice were fed BrdU for 2 weeks before treatment with CD20 or control mAb. Seven d later, spleen and BM cells were harvested and assessed for CD138 and BrdU staining. Despite effective B cell depletion after CD20 mAb treatment, short-lived and long-lived plasma cell numbers were unchanged (Figure 24D).

To directly assess the effect of CD20 mAb treatment on ASC numbers in vivo, naïve mice were given $\mathrm{CD} 20$ or control mAb $7 \mathrm{~d}$ before spleen and BM cells were harvested, with ASC numbers determined by ELISPOT. IgM and IgG ASC numbers in the $\mathrm{BM}$ of mice receiving CD20 and control mAb were comparable (Figure 24E). However, CD20 mAb treatment significantly decreased the number of splenic IgG and IgM ASCs. Thus, BM ASCs expressed insufficient CD20 levels for their depletion in vivo, while some splenic ASCs were depleted. Administration of thioglycollate induces systemic inflammation that enhances B cell depletion by CD20 mAb in vivo (39). However, co-treatment of mice with thioglycollate and CD20 mAb did not enhance the degree of ASC depletion (Figure 24E). 

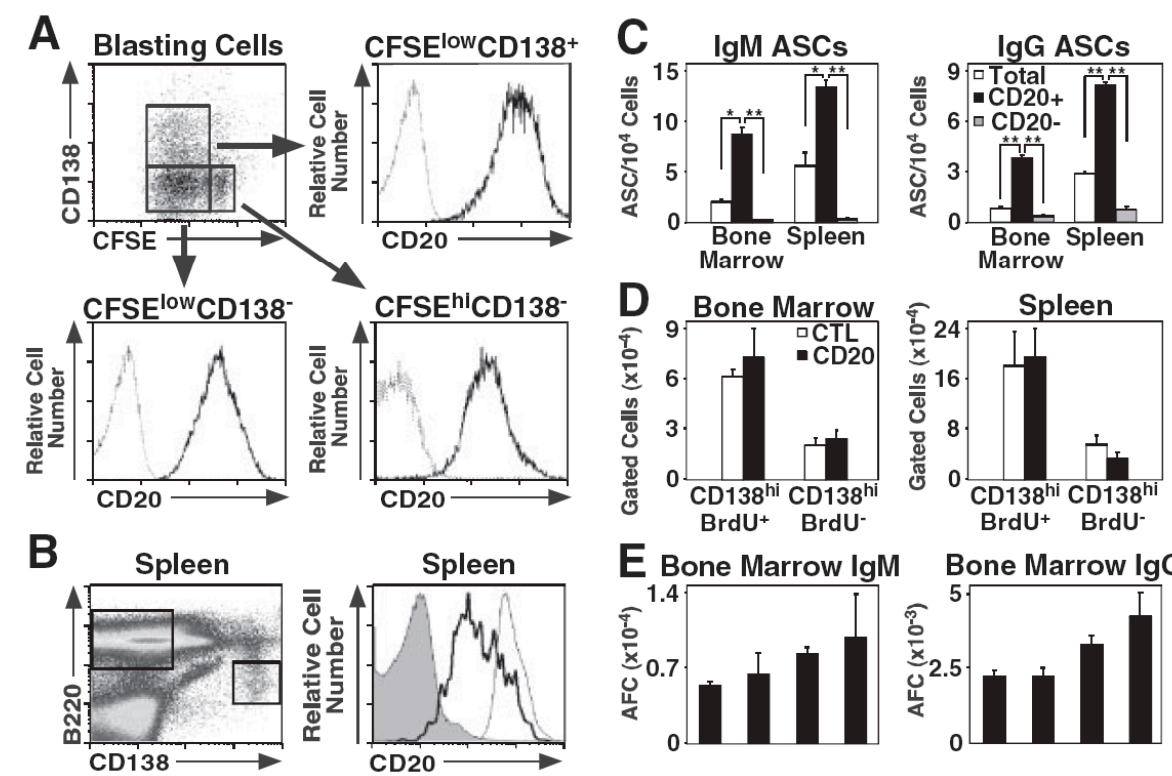

E Bone Marrow IgM
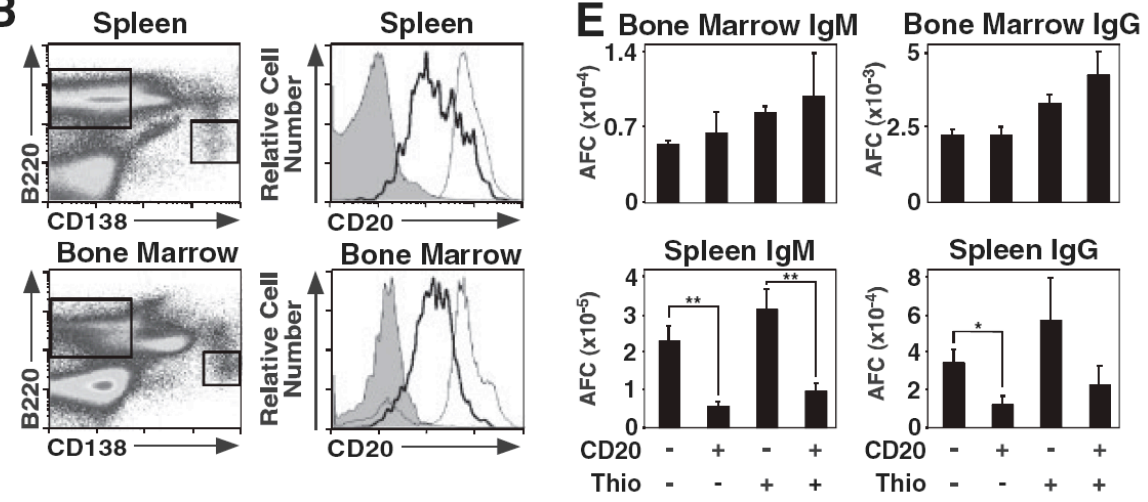

Figure 24: CD20 expression by plasmablasts and ASCs. (A) Representative CD20 expression by purified B $220^{+}$cells that were labeled with CFSE and cultured with LPS for $72 \mathrm{~h}$. CD20 expression by $3 \mathrm{~B}$ cell subpopulations was assessed by immunofluorescence staining with flow cytometry analysis. CD20 expression (heavy lines) and background control anti-mouse IgG2a / c secondary polyclonal Ab (thin lines) staining are indicated for each B cell subset. (B) Spleen and BM B220 ${ }^{\text {low } C D 138 ~}{ }^{\text {high }}$ cells from naive mice express lower levels of CD20 than B220 ${ }^{+} \mathrm{CD} 138^{-}$cells. Dot plots of B220 versus CD138 staining with labeled B220 ${ }^{+}$CD $138^{-}$and $B 220^{\text {low }} C D 138^{\text {high }}$ populations are shown (left panels). B220 ${ }^{+} \mathrm{CD} 138^{-}$(thin line) and $\mathrm{B} 220^{\text {low }} \mathrm{CD} 138^{\text {high }}$ (heavy line) cells were assessed for CD20 expression (right panels). Background control anti-mouse IgG2a/c secondary polyclonal Ab staining is shown (shaded line). A-B) Results represent those obtained in 3 independent experiments. (C) BM and spleen plasma cells express CD20. BM and splenocytes were either unsorted or sorted into $\mathrm{CD} 20^{+}$and $\mathrm{CD} 20^{-}$populations using magnetic beads. Values indicate mean $( \pm$SEM) IgM and IgG ASC numbers per $10^{4}$ cells as quantified by ELISPOT analysis $(n=3)$. (D) CD138 ${ }^{\text {high }}$ cells were maintained in the spleen and BM of CD20 $(n=4)$ or control $(n=4)$ $\mathrm{mAb}$-treated mice. Mice were fed BrdU for 2 weeks before CD20 mAb treatment. Seven $\mathrm{d}$ later, splenic and BM CD138 ${ }^{\text {high }}$ cells were analyzed for BrdU uptake by flow cytometry. Values represent the mean $( \pm S E M)$ total number of cells gated within the indicated population. (E) CD20 mAb depletes splenic but not BM ASCs in vivo. Mice were untreated or given thioglycollate (Thio) before receiving CD20 $(n=4)$ or control $(\mathrm{n}=4) \mathrm{mAb}$. Values indicate mean $( \pm S E M) \operatorname{IgM}$ and IgG ASC numbers contained in a whole spleen or 2 femurs quantified by ELISPOT analysis $7 \mathrm{~d}$ after $\mathrm{mAb}$ injection. A-E) Differences between sample means were significant; ${ }^{*} \mathrm{p}<0.05,{ }^{* *} \mathrm{p}<0.01$. 


\subsubsection{Long-Lived Plasma Cell Depletion}

Since CD20 mAb did not reduce the BM ASC compartment, an alternative approach was tested to determine whether long-lived plasma cells could be depleted. Because BM plasma cells express the LFA-1 and VLA-4 adhesion molecules $(189,194)$, whether function-blocking LFA-1 and VLA-4 mAbs could purge BM ASC from their survival niche (95) was assessed. Co-injection of LFA-1 and VLA-4 mAbs reduced NPspecific IgG ASC numbers by $\sim 75 \%$ in the BM of mice immunized with NP-CGG 6 weeks earlier (Figure 25A). NP-specific ASC numbers in the BM were reduced for up to $28 \mathrm{~d}$, after which NP-specific ASCs returned. BM ASCs were only depleted when LFA-1 and VLA-4 mAbs were used in combination, and similar results were obtained for NPspecific spleen ASCs (data not shown). Thereby, co-blockade of LFA-1 and VLA-4 caused the temporary BM ASC depletion.

Repopulation of the BM with NP-specific ASCs 4 weeks after LFA-1/VLA-4 $\mathrm{mAb}$ injection may have been due to mobilized BM ASCs surviving by circulating within other mouse tissues, followed by ASC re-localization to the BM once the $\mathrm{mAbs}$ were cleared; alternatively, mobilization of ASC from the BM survival niche may have led to ASC death due to loss of survival signals (95), with repopulation of the BM due to new ASC generation from memory B cells. To determine how the BM came to be repopulated with Ag-specific ASC, immunized mice were treated with either CD20 or control mAbs in combination with LFA-1 / VLA-4 blocking mAbs. BM cells were harvested 1, 4, and 10 weeks later. After 1 week of mAb treatment, NP-specific IgG ASC numbers were reduced by $\sim 70 \%(\mathrm{p}<0.02)$ in mice receiving LFA-1/VLA-4 mAbs, or both LFA-1/VLA4 and CD20 mAbs (Figure $25 \mathrm{~B}-\mathrm{C})$. Both total $\left(\mathrm{NP}_{33}\right.$-specific) and high affinity $\left(\mathrm{NP}_{6^{-}}\right.$ specific) IgG ASCs were reduced similarly by LFA-1/VLA-4 mAb treatment. By weeks 4 and $10, C^{2} 0^{+}$B cell depletion prevented the recovery of NP-specific ASC in the BM 
of mice receiving LFA-1 / VLA-4 mAbs, while ASCs repopulated the BM of mice receiving only LFA-1/VLA-4 mAbs. Thus, repopulation of the BM after ASC purging with LFA-1/VLA-4 mAbs was due to new ASC production because CD20+ B cell depletion prevented BM ASC repopulation when given in combination with LFA1/VLA-4 mAbs.

To verify that repopulation of the BM ASC pool in LFA-1/VLA-4 mAb-treated mice was not due to failed depletion of the CD20+ mature/memory B cell pool, the effects of LFA-1 / VLA-4 plus CD20 mAb treatments on B cell depletion in tissues was assessed. Mature BM B cells, and splenic MZ and T2 B cells were cleared 1 and 4 weeks after treating mice with CD20 mAb (Figure 25D-G), but returned by week 10 . By contrast, LFA-1/VLA-4 mAb treatment only depleted mature BM B cells, splenic MZ B cells, and $\sim 50 \%$ of spleen T2 B cells after 1 week, with full repopulation of these subsets by 4 and 10 weeks. One week after LFA-1/VLA-4 mAb treatment, the number of mature spleen B cells increased, but these numbers normalized by 4 weeks after mAb administration. Serum from mice receiving LFA-1/VLA-4 mAbs did not contain detectable levels of rat IgG beyond 1 week post-mAb injection, suggesting that the effect of LFA-1 / VLA-4 mAb treatment does not persist after mAb clearance (data not shown). Finally, consistent with reduced BM plasma cell numbers after combined LFA1/VLA-4 and CD20 mAb treatment, serum NP-specific IgG levels were also significantly reduced (Figure $25 \mathrm{H}$ ). Thus, B cells had the ability to repopulate the BM with ASCs when the plasma cell niche was depleted. However, BM repopulation was abrogated by the concurrent depletion of mature and memory B cells with CD20 mAb. 

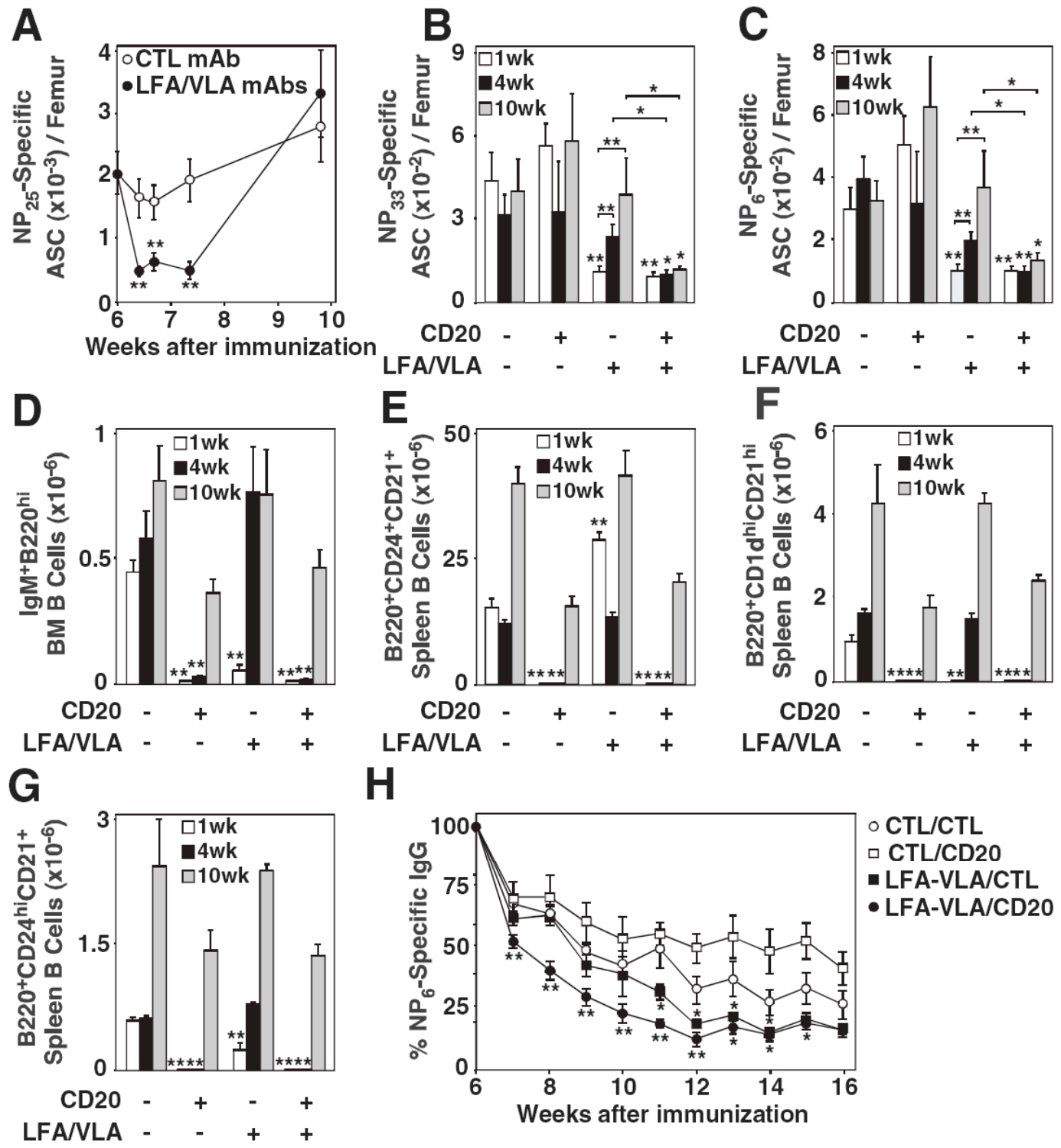

\section{$\mathrm{H}$}

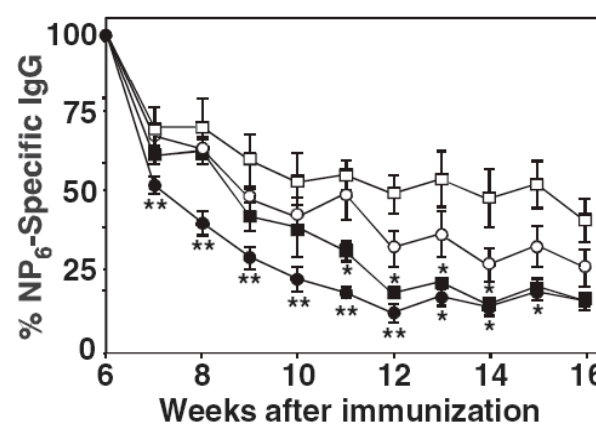

$\circ$ CTL/CTL

$\square$ CTL/CD20

- LFA-VLA/CTL

- LFA-VLA/CD20

Figure 25: BM replenishment after ASC depletion. (A) LFA-1 and VLA-4 mAb treatment depletes Ag-specific plasma cells from the BM. LFA-1 and VLA-4 blocking mAbs $(n \geq 5)$ or control rat IgG $(n \geq 5)$ were administered 6 weeks after NP-CGG immunizations. BM was harvested at the indicated times, with NP-specific ASCs enumerated using ELISPOT assays. Values represent mean $( \pm S E M) \mathrm{NP}_{25}$-specific ASC numbers per femur. $(\mathrm{B}, \mathrm{C}) \mathrm{CD} 20^{+} \mathrm{B}$ cell depletion inhibits repopulation of the $\mathrm{BM}$ by $\mathrm{Ag}$ specific ASCs. Mice were treated with the indicated mAbs 6 weeks after immunization with NP-CGG. $\mathrm{NP}_{33^{-}}$(B) and $\mathrm{NP}_{6}$-specific (C) BM ASCs were enumerated by ELISPOT assay at week $1(n \geq 7)$, week $4(n \geq 3)$, and week $10(n=4)$ after mAb injection. Values represent mean $( \pm S E M) \mathrm{NP}_{33}$ - or $\mathrm{NP}_{6}$-specific ASC numbers per femur. (D-G) B cell subsets within tissues after LFA-1 and VLA-4 mAb treatments. Tissue B cell subsets in immunized mice were analyzed by flow cytometry at week $1(n \geq 3)$, week $4(n \geq 3)$, and week $10(n=4)$ after $\mathrm{mAb}$ injection. Values represent mean $( \pm$ SEM) numbers of 
Figure 25 (Continued) $\mathrm{B} 220^{+} \mathrm{IgM} \mathrm{high}^{\text {high }}$ mature $\mathrm{BM} \mathrm{B}$ cells (D), $\mathrm{B} 220^{+} \mathrm{CD} 21^{+} \mathrm{CD} 24^{+}$mature spleen $B$ cells $(E), B 220^{+} C D 21^{\text {high }} C D 1 d^{+}$spleen MZ B cells (F), and B220 ${ }^{+} \mathrm{CD} 21^{+} \mathrm{CD} 24^{\text {high }}$ spleen T2 B cells $(\mathrm{G})$. (H) Depletion of BM plasma cells decreases $\mathrm{NP}$-specific serum IgG levels. Serum was harvested at the indicated times after immunization from mice treated with control $(n=8)$, CD20 $(n=8)$, LFA-1/VLA-4 $(n=8)$, or both CD20 and LFA-1/VLA-4 ( $\mathrm{n}=7)$ mAbs. $\mathrm{NP}_{6}$-specific serum IgG titers were determined by ELISA. Values at each time point represent the mean percentage of the initial NP-specific titers before mAb injection at week 6. A-H) Differences between sample means for control $\mathrm{mAb}$-treated mice and the indicated samples at the same time point were significant; ${ }^{*} \mathrm{p}<0.05,{ }^{* *} \mathrm{p}<0.01$. 


\subsection{Discussion}

These studies are the first to demonstrate in mice that most nascent humoral immune responses were blocked by $\mathrm{CD} 20^{+} \mathrm{B}$ cell depletion prior to immunization, while pre-developed serum $\mathrm{Ab}$ levels were not affected by either short- or long-term $\mathrm{CD} 20^{+} \mathrm{B}$ cell depletion (Figure 20, Figure 21, Figure 23). Abrogation of primary and secondary Ab responses is explained by the effective depletion of follicular, MZ, B1, GC, and memory B cell subsets (Figure 22, Figure 23, Table 4). Remarkably, long-lived Ab titers persisted in the absence of memory B cell function and recall responses to Ag challenge (Figure 20, Figure 21, Figure 23). Thus, long-lived Ab production does not require persisting Ag to drive the memory B cell pool to chronically generate plasmablasts and short-lived plasma cells as previously suggested (90). Likewise, maintenance of serum Ab levels does not require Ag-independent memory B cell differentiation into plasma cells, as once suggested (91). In fact, inflammation-induced increases in serum Ig levels and hypergammaglobulinemia were inhibited by $\mathrm{CD} 20^{+} \mathrm{B}$ cell depletion without an effect on basal serum Ig levels (Figure 20B, C). Therefore, Ag-independent cytokine or TLR signaling may induce memory or other B cell subsets to differentiate into plasma cells $(91,92)$, but this is not necessary for the maintenance of long-lived serum Ab levels. Thus, long-lived plasma cells or their immediate precursors are indeed intrinsically longlived, and are distinct from the memory B cell pool.

GC B cells, in vitro generated plasmablasts, and some ASCs expressed cell surface CD20 at significant levels (Figure 22A, Figure 24A-B), which allowed their removal from B cell preparations (Figure 24C). By contrast, in vivo CD20 mAb treatment depleted only $\sim 75 \%$ of splenic ASCs, and had no effect on BM ASC numbers (Figure 24E) or established Ag-specific Ab responses (Figure 23, Figure 25). Likewise, CD20 $\mathrm{mAb}$ treatment did not significantly deplete either short-lived CD138 ${ }^{\text {high }} \mathrm{BrdU}^{+}$ 
plasmablasts or long-lived CD138 ${ }^{\text {high }} \mathrm{BrdU}^{-}$plasma cells (160) in vivo (Figure 24D). Spleen ASC depletion despite normal CD138 ${ }^{\text {high }}$ B cell numbers suggests that the use of CD138 as a marker for plasma cells may only be appropriate for a mature CD20 subset of ASCs (195). Nonetheless, the in vivo decrease in splenic ASC numbers after CD20 $\mathrm{mAb}$ treatment is likely due to residual CD20 expression. Alternatively, the depletion of MZ, GC, and memory B cells may prevent the formation of new plasmablasts, while short-lived plasma cells died off naturally during the $7 \mathrm{~d}$ of CD20 mAb depletion. Even though some spleen ASCs were depleted in vivo, they did not appear to contribute significantly to serum Ig levels in comparison with fully mature BM plasma cells. The lack of long-lived BM ASC depletion was most likely attributable to either their lowdensity CD20 expression or the absence of effector monocytes within their microenvironmental niches. Thus, while $\mathrm{CD} 20^{+} \mathrm{B}$ cell depletion was effective at inhibiting primary and secondary humoral immune responses, serologic "memory" resulting from long-lived plasma cells was unaffected.

Function-blocking mAbs against LFA-1 and VLA-4 purged Ag-specific ASCs from the BM (Figure 25A), potentially inducing their death due to loss of survival niche signals (95). The BM was repopulated with plasma cells as soon as the LFA-1 and VLA-4 mAbs were cleared from the circulation. However, co-injection of mice with LFA1/VLA-4 and CD20 mAbs led to prolonged ASC depletion from the BM (Figure 25B-C). Therefore, CD20 $0^{+}$B cells, and most likely memory B cells, were required for repopulating empty plasma cell niches in the BM. The prolonged ASC depletion in LFA-1 / VLA-4 and CD20 mAb co-treated mice was also reflected in serum Ig levels; Ag-specific serum IgG was significantly reduced in mice receiving both CD20 and LFA-1/VLA-4 mAbs (weeks 7-12) compared to mice receiving either $\mathrm{mAb}$ alone. Others have shown that the MZ B cell subset can be specifically mobilized and depleted by LFA-1 / VLA-4 blocking mAbs (196), however, the current studies now demonstrate that LFA-1 and VLA-4 
adhesion molecules also regulate mature B cell and plasma cell localization within the BM. Moreover, this study is the first to show that Ag-specific long-lived plasma cells can be purged from the BM by blocking adhesion receptor function, with memory B cells required for repopulation of a plasma cell-deficient BM. Therefore, long-lived plasma cells and memory B cells contribute in interdependent ways to long-lived serological memory; mature and memory B cells are not required for maintaining BM plasma cell numbers, but are required for repopulation of plasma cell-deficient BM through Agdependent (8) or -independent (91) mechanisms. Thus, CD20 mAb treatment in combination with LFA-1 / VLA-4 blocking mAbs could provide a new therapeutic avenue for BM plasma cell depletion that may also lead to the identification of additional similar strategies for depleting plasma cells from other tissues.

CD20 $0^{+}$B cell depletion did not inhibit IgM responses to TNP-LPS or DNP-KLH immunizations, while IgM responses to DNP-Ficoll were significantly inhibited by CD20 $\mathrm{mAb}$ treatment (Figure 21 ). That CD20 mAb treatment rapidly and effectively depletes MZ B cells but not peritoneal B cells (39) indicates that peritoneal B cells may preferentially generate IgM responses to TI-1 and TD Ags. IgM Ab generation by peritoneal B cells is further supported by the observation that primary IgM Ab responses were not generated in CD20 mAb-treated mice after rechallenge with DNP-KLH (Figure 21) since CD20 mAb eventually reduces peritoneal B cell numbers by the time that these mice were boosted with Ag (39). CD20 mAb plus thioglycollate treatments also reduced basal serum IgM Ab levels by up to half (Figure 20B), most likely due to the depletion of peritoneal B cells and their natural Ab products (197). Otherwise, CD20 mAb plus thioglycollate treatments did not significantly alter BM and spleen ASC numbers or basal serum Ig levels (Figure 24), arguing that peritoneal B cells only produce a portion of serum IgM and relatively little IgG. Thereby, augmenting the innate immune response using agents like thioglycollate to mediate more effective B cell depletion may not 
augment the reduction of serum Ig levels. Thus, the majority of serum $\mathrm{Ab}$ appears to be continually produced by long-lived plasma cells that do not require replenishment once generated.

The absence of Ag-specific Ab isotype switching and IgG-subclass Ab responses to TI-1, TI-2, and TD Ags in CD20 mAb-treated mice is likely due to the depletion of GC B cells and their precursors (Figure 21, Figure 22). Most B cells with a GC phenotype and histologically recognizable GCs were depleted following $7 \mathrm{~d}$ of CD20 mAb treatment, with at least 70\% of GC B cells depleted in immunized mice (Figure 22B-D, ref. 39). Thereby, GC-dependent clonal expansion, somatic hypermutation, and isotype switching are unlikely to occur to a significant extent following CD20 mAb treatment. CD20 mAb treatment also prevented high-affinity Ag-specific Ab formation and the expansion of ASCs in BM and spleens of recipient mice during adoptive transfer experiments. Thus, memory B cells expressed sufficient levels of CD20 to be effectively targeted in vivo, with memory Ab responses virtually eliminated by CD20 mAb treatments in vivo (Figure 23). Thereby, the elimination of GC and memory B cells explains the absence of isotype-switched Ab responses in CD20 mAb-treated mice.

That CD20 mAb treatment impairs primary and recall Ab responses while maintaining serum Ig levels in mice is consistent with studies in patients receiving Rituximab, a chimeric anti-human CD20 mAb. This mAb can cause decreased $\mathrm{Ab}$ responses to some recall Ags (198), while total Ig levels in lymphoma and rheumatoid arthritis patients generally remain within the normal range (198-201). Rituximab treatment also decreases primary Ab responses and class switching in baboons (202). While anti-bacterial $\mathrm{Ab}$ levels are preserved in autoimmune disease patients following Rituximab-mediated B cell depletion (201), a positive clinical response is associated with a significant fall in autoAb levels in some patients (203-207), but not all (208). However, these conclusions are complicated by the fact that most patients also receive 
supplementary immunosuppressive therapies and the full extent of B cell clearance in humans given Rituximab is unknown. In autoimmune mice, continuous CD20 mAb treatment does not affect circulating Ig or autoAb levels $(43,133)$. Thus, CD20 mAb therapy may not be beneficial if pathogenic autoAb-secreting cells are long-lived or do not express CD20. However, CD20 mAb treatment may deplete memory B cells or the precursors of pathogenic auto Ab secreting plasma cells, thereby reducing the sources of new Ab-secreting plasma cells in autoimmune disease where autoreactive memory B cells may be continually "tickled" with Ag, leading to perpetual plasma cell differentiation. It is important to note that the effects of Rituximab in human patients may not completely recapitulate the results detailed in this study. Rituximab and the anti-mouse CD20 mAb utilized in this study are vastly distinct mAbs, with potentially different binding capabilities and effects in vivo $(39,71,72)$.

The current studies demonstrate that depleting the majority of peripheral B cells by CD20 mAb treatment does not have a dramatic negative effect on pre-existing Ab levels because plasma cells are long-lived and survive independent of repopulation by the B cell compartment. These results contrast with significant reductions in serum Ab levels within two weeks of $\mathrm{CD} 19^{+}$B cell depletion in mouse models (156), consistent with the measured half-lives of serum antibodies (209). Thus, serum Ab deficiency may not be a significant consequence of long-term B cell depletion using CD20 mAbs, except when new Ags and pathogens are encountered. However, alternative therapeutic strategies for depleting long-lived plasma cells will have to be devised to combat the negative clinical implications of humoral memory once initiated. Thus, a balance between effective B cell depletion, therapeutic benefit, and immunodeficiency will also need to be established as more potent $B$ cell depletion strategies move into clinical practice. 


\section{Discussion}

The studies described in this dissertation demonstrate that B cells function through multiple effector mechanisms to influence the course and intensity of immune responses: the promotion of cellular immune responses, the negative regulation of cellular immune responses, and the production and maintenance of long-lived Ag-specific serum $\mathrm{Ab}$ titers (Figure 26). Therefore, each of these three B cell effector mechanisms can contribute independently or in concert with the other mechanisms to clear pathogens or cause tissue damage during autoimmunity.

\subsection{B Cell Functions during Autoimmune Disease}

Autoimmunity occurs when B cell negative selection in the BM fails and tolerance to self Ags is broken. Autoimmunity also results when genes involved in regulating the lifespan of a B cell become dysregulated, thereby promoting the survival of autoreactive B cells. Self-reactive mature B cells recognize self Ag, clonally expand, and contribute to tissue destruction and disease, and carry out multiple effector functions. Self-reactive B cells may also differentiate into long-lived memory B cells and plasma cells that allow disease to be maintained in the host indefinitely.

\subsubsection{B Cell Promotion of Autoimmune Disease}

The studies described in this dissertation demonstrate that B cells have the capability to promote cellular immune responses. While these studies did not directly assess the mechanisms by which B cells promote $\mathrm{T}$ cell immune responses, this has been studied in other mouse models. B cells are most likely presenting Ags and are thereby inducing $\mathrm{CD}^{+} \mathrm{T}$ cell activation, since B cells can present Ags as efficiently as other APCs (138). Furthermore, B cell MHC class II expression is required for maximal Agspecific $\mathrm{CD}^{+} \mathrm{T}$ cell expansion, $\mathrm{CD} 4^{+}$memory formation, and $\mathrm{CD} 4^{+} \mathrm{T}$ cell cytokine 
production in vivo $(19,20)$. When B cells present Ag to T cells, it is likely that the B cells provide costimulatory signals as well (30). Further, cytokine production by B cells can positively influence $\mathrm{T}$ cell responses, since B cells produce a number of proinflammatory cytokines, including $\mathrm{TNF} \alpha, \mathrm{IFN} \gamma$, and IL-12, which can promote cell-mediated immunity. Thereby, B cells may utilize multiple mechanisms to promote immune responses and contribute to autoimmune disease.

Thus, B cells can promote autoimmune disease by promoting cellular immune responses. Self-reactive B cells escape the BM where they would normally be negatively selected. They migrate to peripheral tissues where they encounter their cognate self Ag and become activated and clonally expand. These B cells may also phagocytose this Ag, process it, and present it to $\mathrm{T}$ cells in their vicinity. If a CD4 ${ }^{+} \mathrm{T}$ cell recognizes the peptide presented by the B cell and also receives the proper costimulatory signals from the B cell (CD80 / 86, OX40L, etc), it becomes activated and carries out its effector functions to cause inflammation and tissue damage. Therefore, $\mathrm{B}$ cells can activate CD4 ${ }^{+}$ $\mathrm{T}$ cells to induce autoimmune disease.

\subsubsection{B Cell Negative Regulation of Autoimmune Disease}

These studies also highlighted the fact that while in some situations B cells promote immune responses, B cells function to inhibit cell-mediated immunity in other situations. It is now understood that a subset of B cells has the capacity to secrete IL-10 (B10 cells), and these cells can regulate immune responses in an IL-10 dependent manner $(58,68,73,81,116)$. Regulatory B10 cells have been demonstrated to function during other mouse models of disease or immunity, including EAE, CIA, and DTH, and the adoptive transfer of these cells can suppress immune responses $(58,68,73,81,116)$.

In the context of autoimmune disease, regulatory B10 cells may function in several ways. It is known that B10 cells can function in an Ag-specific manner. Thus, 
B10 cells may secrete IL-10 at the same time they present self Ag to auto-reactive CD4 ${ }^{+}$ $\mathrm{T}$ cells, thereby directly inhibiting the T cell response. Alternatively, IL-10 produced by B cells may act indirectly on T cells by impairing DC-, macrophage-, or even B cellmediated activation of T cells. B cell-derived IL-10 may also function in a more global manner to systemically to suppress immunity, in addition to B cell release of IL-10 in microenvironments to influence single or small numbers of target cells. Any or all of these potential mechanisms may occur simultaneously, and the manner in which regulatory B10 cells function may differ between experimental models and autoimmune diseases. Thus, B cells can function to suppress immune responses during autoimmunity.

\subsubsection{Ab Functions during Autoimmunity}

$\mathrm{Ab}$ functions as both a natural barrier to infection and as the humoral component of adaptive responses to pathogens. However, $\mathrm{Ab}$ alone is often unable to efficiently or completely clear pathogens or cause pathology during autoimmunity. Instead, synergy with the cellular components of the immune system is required for optimal immune responses. Thus, depletion of both $\mathrm{B}$ cells and $\mathrm{Ab}$ was necessary to prevent chronic kidney allograft rejection in the current studies; mature B cell depletion alone did not affect rejection. These results indicate that both $\mathrm{B}$ cells and $\mathrm{Ab}$ are required for allograft destruction during chronic kidney allograft rejection. Similar results have been obtained in a mouse model of lupus (210). B cells are absolutely required for lupus disease in MRL/lpr mice, as disease does not develop in MRL/lpr mice genetically deficient in B cells. Transgenic mIgM.MRL/lpr mice that express membrane Ig, but do not secrete circulating Ig, do develop nephritis and vasculitis. However, disease is less severe in these transgenic mice compared to MRL/lpr mice. Thus, both $\mathrm{Ab}$ and positive contributions by B cells are required for optimal autoimmune disease. 
While $\mathrm{Ab}$ is required for disease pathogenesis in some autoimmune syndromes, $\mathrm{Ab}$ is dispensable for disease pathogenesis in others. In the studies outlined in this dissertation, $\mathrm{Ab}$ is not detected in the B16 melanoma tumor model, nor is $\mathrm{Ab}$ required for skin allograft rejection, even though it is produced during the allograft immune response. In other mouse models of autoimmune disease, autoAbs alone are unable to initiate autoimmune disease (128). In human autoimmune diseases including lupus, autoAbs are important for disease diagnosis and prognostic purposes, but their serum levels do not always correlate with disease activity. In other autoimmune disorders such as autoimmune blistering diseases, auto Ab levels can highly correlate with clinical disease activity (128). Still, the presence of autoAbs in these diseases does not mean that the Abs are pathogenic. Collectively, the current results and previous reports indicate that while $\mathrm{Ab}$ can induce pathology on its own and is an important component of the autoimmune response, other functions of B cells and other facets of the immune system are also required in addition to $\mathrm{Ab}$ for optimal immune responses.

\subsection{B cell effector mechanisms are not mutually exclusive}

Since no single B cell effector mechanism discussed above can completely account for the pathology that results from autoimmune disease, any or all of these potential mechanisms may function simultaneously during the course of an immune response. Thus, B cells likely simultaneously differentiate into Ab-secreting plasma cells that secrete long-lived pathogenic $\mathrm{Ab}$, promote immune responses through $\mathrm{Ag}$ presentation and the provision of costimulatory molecules, and negatively regulate immune responses through the production of IL-10. However, one or more of these effector mechanisms will dominate in the context of each model of disease. The additive effects of each of these distinct mechanisms will ultimately determine the outcome of the immune response. 
Thus, a delicate balance must exist between the positive and negative regulatory functions of B cells during normal immune responses and autoimmune disease. 


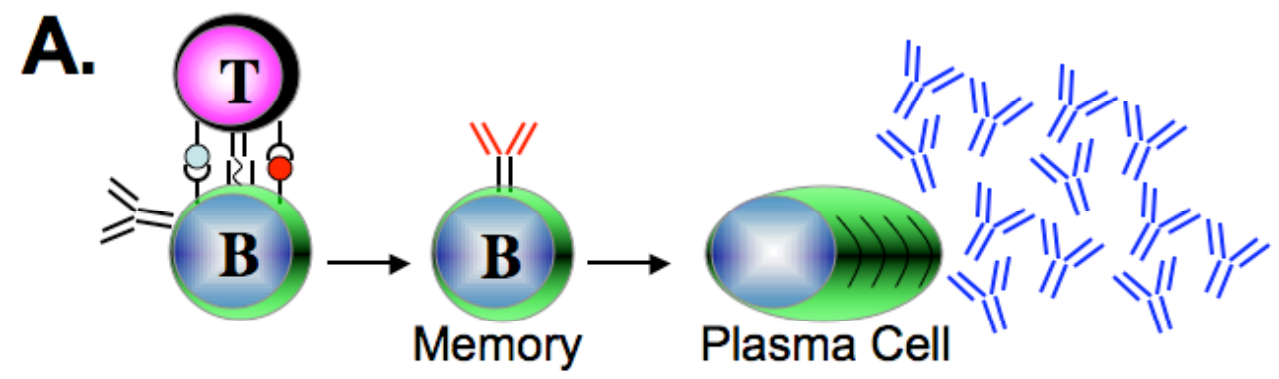

B.
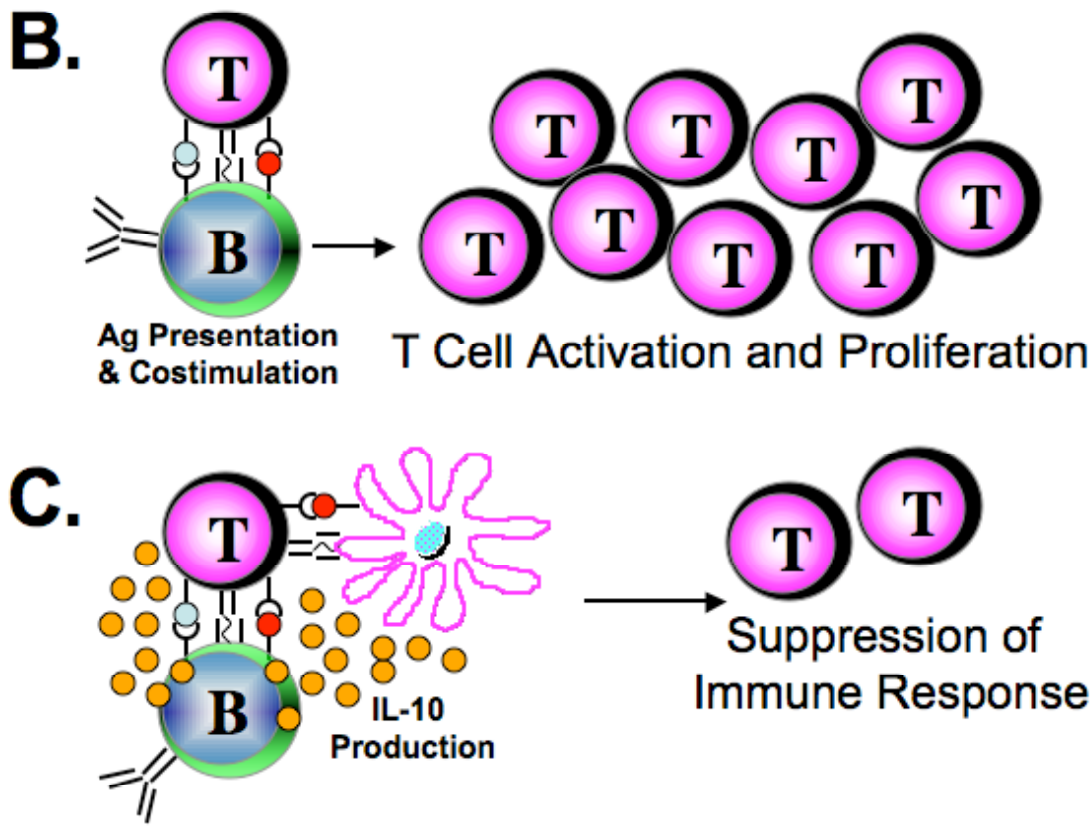

Figure 26. B cell effector mechanisms during immune responses. B cells (A) differentiate into plasma cells that are long-lived and secrete pathogenic $A b,(B)$ promote immune responses by presenting Ag and providing costimulatory signals, and (C) suppress immune responses though the production of IL-10. 


\subsection{Remaining Questions Regarding B Cell Effector Functions 6.3.1 Potential Factors Influencing How B Cells Function during Autoimmunity}

The current studies demonstrate that B cells contribute in multiple ways during the course of an immune response. However, the effector functions carried out by B cells vary widely between experimental models. In some models, including DTH and skin allograft rejection, B cells function as potent suppressor cells to inhibit immune responses. In other models, such as diabetes, arthritis, and chronic allograft rejection, B cells promote immune responses. Adding to the complexity, B cells may function to both promote and inhibit disease in some cases, such as in the EAE model. Further, Ab production by terminally-differentiated B cells is also a pathogenic component of some of these models, such as lupus, arthritis, and chronic allograft rejection. Finally, B cells may play no obvious role in other diseases, such as acute cardiac allograft rejection. What factors determine the role or roles that B cells play in different diseases?

The dose of Ag present during B cell activation may influence the role that B cells play during an immune response. For example, B cells present Ags and autoAgs efficiently when the Ags are present at low concentrations $(27,51)$. Specifically, B cells are not required for optimal $\mathrm{CD}^{+} \mathrm{T}$ cell activation at high $\mathrm{Ag}$ concentrations, while this responsibility is shared by B cells and DCs when Ag or autoAg concentrations are low (27). This may be the case in autoimmunity, where small doses of self Ag may be released when cells die before they can be efficiently cleared by the innate immune system.

The nature of the Ag recognized by B cells may also influence the manner in which the B cell directs the immune response. For example, the immunogenicity of the Ag or the strength of the BCR signal induced by Ag binding may determine how a B cell responds. MZ B cells express unmutated BCRs, are enriched for poly-reactive and auto- 
reactive B cell clones, and can respond to Ag in the absence of T cell help (211-213). Thus, these B cells may recognize low-affinity self or foreign Ag and respond by rapidly making $\mathrm{Ab}$ or presenting these Ags to $\mathrm{T}$ cells to elicit a cellular immune response.

The subset of B cells that responds, either initially or later in the course of disease, may impact the role that B cells play during the course of an immune response. For example, MZ B cells capture, process, and present Ag to $\mathrm{CD}^{+}{ }^{+} \mathrm{T}$ cells in a much more efficient manner than follicular B cells (214) and rapidly differentiate into plasma cells (211). Thus, if MZ B cells are the first to respond to a self Ag, it is likely that B cells will promote autoimmunity and $\mathrm{Ab}$ will be generated. However, if regulatory B10 cells are involved in the early detection of Ag, it is likely that B cell-mediated suppression of the subsequent autoimmune response will occur. However, in reality, multiple subsets of B cells likely respond to pathogens and autoAgs in vivo. Thus, whichever B cell subset dominates the response will likely direct the overall influence of B cells over the course of the immune response.

The inflammatory status of the host at the time of Ag or pathogen challenge may impact the manner in which B cells function. B cells may be affected by the cytokines and other factors released by components of the innate immune system, which can modulate B cell function. B cells themselves express innate immune receptors, such as TLRs, which are present on invading pathogens as well as in adjuvants used to induce autoimmune disease in mouse models. Since TLR ligand inflammatory signals induce the regulatory B10 cell subset to secrete immunosuppressive IL-10, fits that regulatory B10 cells play such an important role during EAE, which is initiated by immunization with Ag in adjuvant containing TLR ligands. Further, intact TLR signaling pathways and TLR-2 / 4 in B cells are required for recovery from EAE $(55,74)$. By contrast, B cells may function to promote immune responses in models in which inflammation is not a factor. 
For example, few innate signals are present in the B16 melanoma model, in which B cells function to promote tumor immunity.

B cells may function to both inhibit and promote the same immune response, depending on the timing of the immune response. The best example of this phenomenon occurs in the EAE mouse model. B cell depletion before EAE initiation leads to more severe disease, indicating that regulatory B10 cells function during the initial acute phase of disease. However, B cell depletion after EAE is established leads to reduced pathology, indicating that B cells promote disease in the later phases (58). Thus, B cells may function in a regulatory manner in the acute phases of immune responses (and autoimmunity) to prevent excess immune response (or the initiation of autoimmunity). However, late phases of immune responses and autoimmunity may be characterized by the promotion of immunity by Ag-presenting B cells.

\subsubsection{Do B Cell Subsets Carry Out These Effector Functions?}

It remains unknown whether the $\mathrm{Ab}$ production, $\mathrm{Ag}$ presentation, and regulatory effector mechanisms are each carried out by distinct subsets of B cells, or if all B cells have the capacity to perform each of these functions. Currently, studies indicate that regulatory B10 cells are a unique and distinct subset of cells that produce IL-10, since most other B cells do not secrete this immunoinhibitory cytokine. However, whether these regulatory $\mathrm{B}$ cells can also terminally differentiate to produce $\mathrm{Ab}$ is unclear. Similarly, it is unknown whether a B cell is instructed to only perform one of these effector functions, or if B cells are more plastic and can both present Ag and produce IL10 to modulate immune responses and autoimmunity. Finally, whether cells that performed these effector functions can form memory cells is also unknown. 


\subsection{B Cell Effector Functions in Human Autoimmune Disease}

While the current studies in this dissertation addressed the roles of B cells in mice, the findings are applicable to human autoimmune disease and correlate well with results seen in human studies. Human B cells can also function to promote $\mathrm{CD}^{+} \mathrm{T}$ cell responses, because CD20 mAb (Rituximab) treatment in human patients has been shown to induce disease remission in pemphigus vulgaris and lupus patients with decreases in autoreactive $\mathrm{T}$ cell frequencies $(31,215-218)$, even though effects on auto $\mathrm{Ab}$ titers were nonexistent or variable. Therefore, targeting B cells for depletion in autoimmune disease may prove to be an effective treatment for autoimmune diseases where B cells function to promote disease. Conversely, B cells may be manipulated to be used as adjuvants in situations in which the enhancement of the immune response and $\mathrm{CD}^{+} \mathrm{T}$ cell activation is desired, such as loading B cells with Ag in vaccination strategies. However, since it is clear that B cells can also negatively regulate immune responses, care must be taken in designing these therapies, as B cell depletion strategies may result in the enhancement of certain autoimmune diseases $(58,180)$. By contrast, the development of methods to expand or hyper-activate regulatory B10 cells may prove effective in treating autoimmune conditions.

\subsection{Conclusions}

For decades, the only effector mechanism attributed to B cells was their ability to terminally differentiate into plasma cells that secrete $\mathrm{Ab}$, which functions to clear pathogens and can cause tissue damage during autoimmunity. Now, it is clear that B cell effector mechanisms go far beyond Ab production. Depending on the situation, B cells may not only produce $\mathrm{Ab}$, but they may also promote cellular immune responses and function as adjuvants for $\mathrm{CD} 4^{+} \mathrm{T}$ cell activation. By contrast, some B cells are also capable of inhibiting immune responses through their regulatory functions. Thus, while 
questions regarding B cell effector mechanisms remain, further understanding of the balance between the multiple contributions of B cells during immunity will allow the generation of new treatments for patients suffering from immune-mediated diseases, such as autoimmunity, cancer, and allograft rejection. 


\section{References}

1. Hoebe, K., E. Janssen, and B. Beutler. 2004. The interface between innate and adaptive immunity. Nat. Immunol. 5:971-974.

2. DiLillo, D. J., Y. Hamaguchi, Y. Ueda, K. Yang, J. Uchida, K. M. Haas, G. Kelsoe, and T. F. Tedder. 2008. Maintenance of long-lived plasma cells and serological memory despite mature and memory B cell depletion during CD20 immunotherapy in mice. J. Immunol. 180:361-371.

3. Fazilleau, N., L. Mark, L. J. McHeyzer-Williams, and M. G. McHeyzer-Williams. 2009. Follicular helper T cells: lineage and location. Immunity 30:324-335.

4. Lefrancois, L., and J. J. Obar. Once a killer, always a killer: from cytotoxic T cell to memory cell. Immunol. Rev. 235:206-218.

5. LeBien, T. W., and T. F. Tedder. 2008. B-lymphocytes: How they develop and function. Blood 112:1570-1579.

6. Ho, F., J. E. Lortan, I. C. MacLennan, and M. Khan. 1986. Distinct short-lived and long-lived antibody-producing cell populations. Eur. J. Immunol. 16:1297-1301.

7. Jacob, J., and G. Kelsoe. 1992. In situ studies of the primary immune response to (4-hydroxy-3-nitrophenyl)acetyl. II. A common clonal origin for periarteriolar lymphoid sheath-associated foci and germinal centers. J. Exp. Med. 176:679-687.

8. Takahashi, Y., P. R. Dutta, D. M. Cerasoli, and G. Kelsoe. 1998. In situ studies of the primary immune response to (4-hydroxy-3-nitrophenyl)acetyl. V. Affinity maturation develops in two stages of clonal selection. J. Exp. Med. 187:885-895.

9. McHeyzer-Williams, M. G., M. J. McLean, P. A. Lalor, and G. J. V. Nossal. 1993. Antigen-driven B cell differentiation in vivo. J. Exp. Med. 178:295-305.

10. McHeyzer-Williams, M. G., G. J. Nossal, and P. A. Lalor. 1991. Molecular characterization of single memory B cells. Nature 350:502-505.

11. Lalor, P. A., G. J. V. Nossal, R. D. Sanderson, and M. G. McHeyzer-Williams. 1992. Functional and molecular characterization of single, (4-hydroxy-3nitropheny)acetyl (NP)-specific, $\mathrm{I}_{8} \mathrm{G}_{1}{ }^{+} \mathrm{B}$ cells from antibody-secreting and memory B cell pathways in the C57BL/ 6 immune response to NP. Eur. J. Immunol. 22:3001-3011.

12. McHeyzer-Williams, L. J., and M. G. McHeyzer-Williams. 2005. Antigen-specific memory B cell development. Annu. Rev. Immunol. 23:487-513.

13. Kurt-Jones, E. A., D. Liano, K. A. HayGlass, B. Benacerraf, M. S. Sy, and A. K. Abbas. 1988. The role of antigen-presenting B cells in T cell priming in vivo. Studies of B cell-deficient mice. J. Immunol. 140:3773-3778. 
14. Constant, S., N. Schweitzer, J. West, P. Ranney, and K. Bottomly. 1995. B lymphocytes can be competent antigen-presenting cells for priming $\mathrm{CD} 4^{+} \mathrm{T}$ cells to protein antigens in vivo. J. Immunol. 155:3734-3741.

15. Ron, Y., P. De Baetselier, J. Gordon, M. Feldman, and S. Segal. 1981. Defective induction of antigen-reactive proliferating T cells in B cell-deprived mice. Eur. J. Immunol. 11:964-968.

16. Ron, Y., and J. Sprent. 1987. T cell priming in vivo: a major role for B cells in presenting antigen to T cells in lymph nodes. J. Immunol. 138:2848-2856.

17. Janeway, C. A., Jr., J. Ron, and M. E. Katz. 1987. The B cell is the initiating antigen-presenting cell in peripheral lymph nodes. J. Immunol. 138:1051-1055.

18. Kitamura, D., J. Roes, R. Kuhn, and K. Rajewsky. 1991. A B cell-deficient mouse by trageted disruption of the membrane exon of the immunoglobulin $\mu$ chain gene. Nature 350:423-426.

19. Linton, P. J., J. Harbertson, and L. M. Bradley. 2000. A critical role for B cells in the development of memory CD4 cells. J. Immunol. 165:5558-5565.

20. Crawford, A., M. Macleod, T. Schumacher, L. Corlett, and D. Gray. 2006. Primary $\mathrm{T}$ cell expansion and differentiation in vivo requires antigen presentation by B cells. J. Immunol. 176:3498-3506.

21. Liu, Y., Y. Wu, L. Ramarathinam, Y. Guo, D. Huszar, M. Trounstine, and M. Zhao. 1995. Gene-targeted B-deficient mice reveal a critical role for B cells in the CD4 T cell response. Int. Immunol. 7:1353-1362.

22. Epstein, M. M., F. Di Rosa, D. Jankovic, A. Sher, and P. Matzinger. 1995. Successful T cell priming in B cell-deficient mice. J. Exp. Med. 182:915-922.

23. Moulin, V., F. Andris, K. Thielemans, C. Maliszewski, J. Urbain, and M. Moser. 2000. B lymphocytes regulate dendritic cell (DC) function in vivo: increased interleukin 12 production by DCs from B cell-deficient mice results in T helper cell type 1 deviation. J. Exp. Med. 192:475-482.

24. Shen, H., J. K. Whitmire, X. Fan, D. J. Shedlock, S. M. Kaech, and R. Ahmed. 2003. A specific role for B cells in the generation of CD8 T cell memory by recombinant Listeria monocytogenes. J. Immunol. 170:1443-1451.

25. Lassila, O., O. Vainio, and P. Matzinger. 1988. Can B cells turn on virgin T cells? Nature 334:253-255.

26. Ronchese, F., and B. Hausmann. 1993. B lymphocytes in vivo fail to prime naive T cells but can stimulate antigen-experienced T lymphocytes. J. Exp. Med. 177:679690.

27. Bouaziz, J. D., K. Yanaba, G. M. Venturi, Y. Wang, R. M. Tisch, J. C. Poe, and T. F. Tedder. 2007. Therapeutic B cell depletion impairs adaptive and autoreactive $\mathrm{CD}^{+} \mathrm{T}$ cell activation in mice. Proc. Natl. Acad. Sci. USA 104:20882-20887. 
28. Garside, P., E. Ingulli, R. R. Merica, J. G. Johnson, R. J. Noelle, and M. K. Jenkins. 1998. Visualization of specific B and T lymphocyte interactions in the lymph node. Science 281:96-99.

29. Rodriguez-Pinto, D., and J. Moreno. 2005. B cells can prime naive CD4 ${ }^{+} \mathrm{T}$ cells in vivo in the absence of other professional antigen-presenting cells in a CD154CD40-dependent manner. Eur J Immunol 35:1097-1105.

30. Linton, P. J., B. Bautista, E. Biederman, E. S. Bradley, J. Harbertson, R. M. Kondrack, R. C. Padrick, and L. M. Bradley. 2003. Costimulation via OX40L expressed by B cells is sufficient to determine the extent of primary CD4 cell expansion and Th2 cytokine secretion in vivo. J. Exp. Med. 197:875-883.

31. Sfikakis, P. P., J. N. Boletis, S. Lionaki, V. Vigklis, K. G. Fragiadaki, A. Iniotaki, and H. M. Moutsopoulos. 2005. Remission of proliferative lupus nephritis following B cell depletion therapy is preceded by down-regulation of the $\mathrm{T}$ cell costimulatory molecule CD40 ligand: an open-label trial. Arthritis Rheum. 52:501513.

32. Tumanov, A., D. Kuprash, M. Lagarkova, S. Grivennikov, K. Abe, A. Shakhov, L. Drutskaya, C. Stewart, A. Chervonsky, and S. Nedospasov. 2002. Distinct role of surface lymphotoxin expressed by B cells in the organization of secondary lymphoid tissues. Immunity 17:239-250.

33. Gonzalez, M., F. Mackay, J. L. Browning, M. H. Kosco-Vilbois, and R. J. Noelle. 1998. The sequential role for lymphotoxin and B cells in the development of splenic follicles. J. Exp. Med. 187:997-1007.

34. Joao, C., B. M. Ogle, C. Gay-Rabinstein, J. L. Platt, and M. Cascalho. 2004. B celldependent TCR diversification. J. Immunol. 172:4709-4716.

35. AbuAttieh, M., M. Rebrovich, P. J. Wettstein, Z. Vuk-Pavlovic, A. H. Limper, J. L. Platt, and M. Cascalho. 2007. Fitness of cell-mediated immunity independent of repertoire diversity. J. Immunol. 178:2950-2960.

36. Ngo, V. N., R. J. Cornall, and J. G. Cyster. 2001. Splenic T zone development is B cell dependent. J. Exp. Med. 194:1649-1660.

37. Crowley, M. T., C. R. Reilly, and D. Lo. 1999. Influence of lymphocytes on the presence and organization of dendritic cell subsets in the spleen. J. Immunol. 163:4894-4900.

38. Golovkina, T. V., M. Shlomchik, L. Hannum, and A. Chervonsky. 1999.

Organogenic role of B lymphocytes in mucosal immunity. Science 286:1965-1968.

39. Hamaguchi, Y., J. Uchida, D. W. Cain, G. M. Venturi, J. C. Poe, K. M. Haas, and T. F. Tedder. 2005. The peritoneal cavity provides a protective niche for B1 and conventional B lymphocytes during anti-CD20 immunotherapy in mice. J. Immunol. 174:4389-4399. 
40. Macaulay, A. E., R. H. DeKruyff, C. C. Goodnow, and D. T. Umetsu. 1997. Antigen-specific B cells preferentially induce $\mathrm{CD} 4^{+} \mathrm{T}$ cells to produce IL-4. J. Immunol. 158:4171-4179.

41. Liu, Q., Z. Liu, C. T. Rozo, H. A. Hamed, F. Alem, J. F. Urban, Jr., and W. C. Gause. 2007. The role of B cells in the development of CD4 effector T cells during a polarized Th2 immune response. J. Immunol. 179:3821-3830.

42. Harris, D. P., L. Haynes, P. C. Sayles, D. K. Duso, S. M. Eaton, N. M. Lepak, L. L. Johnson, S. L. Swain, and F. E. Lund. 2000. Reciprocal regulation of polarized cytokine production by effector B and T cells. Nat. Immunol.:475-482.

43. Yanaba, K., Y. Hamaguchi, G. M. Venturi, D. A. Steeber, E. W. St.Clair, and T. F. Tedder. 2007. B cell depletion delays collagen-induced arthritis in mice: arthritis induction requires synergy between humoral and cell-mediated immunity. $J$. Immunol. 179:1369-1380.

44. Silveira, P. A., and S. T. Grey. 2006. B cells in the spotlight: innocent bystanders or major players in the pathogenesis of type 1 diabetes. Trends. Endocrinol. Metabol. 17:128-135.

45. Fox, C. J., and J. S. Danska. 1998. Independent genetic regulation of T-cell and antigen-presenting cell participation in autoimmune islet inflammation. Diabetes 47:331-338.

46. Signore, A., P. Pozzilli, E. A. Gale, D. Andreani, and P. C. Beverley. 1989. The natural history of lymphocyte subsets infiltrating the pancreas of NOD mice. Diabetologia 32:282-289.

47. Kendall, P. L., G. Yu, E. J. Woodward, and J. W. Thomas. 2007. Tertiary lymphoid structures in the pancreas promote selection of B lymphocytes in autoimmune diabetes. J. Immunol. 178:5643-5651.

48. Serreze, D. V., S. A. Fleming, H. D. Chapman, S. D. Richard, E. H. Leiter, and R. M. Tisch. 1998. B lymphocytes are critical antigen-presenting cells for the initiation of $\mathrm{T}$ cell-mediated autoimmune diabetes in nonobese diabetic mice. J. Immunol. 161:3912-3918.

49. Falcone, M., J. Lee, G. Patstone, B. Yeung, and N. Sarvetnick. 1998. B lymphocytes are crucial antigen-presenting cells in the pathogenic autoimmune response to GAD65 antigen in nonobese diabetic mice. J. Immunol. 161:11631168.

50. Noorchashm, H., Y. K. Lieu, N. Noorchashm, S. Y. Rostami, S. A. Greeley, A. Schlachterman, H. K. Song, L. E. Noto, A. M. Jevnikar, C. F. Barker, and A. Naji. 1999. I- $\mathrm{A}^{\mathrm{g} 7}$-mediated antigen presentation by $\mathrm{B}$ lymphocytes is critical in overcoming a checkpoint in $\mathrm{T}$ cell tolerance to islet $\beta$ cells of nonobese diabetic mice. J. Immunol. 163:743-750.

51. Xiu, Y., C. P. Wong, Y. Hamaguchi, Y. Wang, S. Pop, R. M. Tisch, and T. F. Tedder. 2008. B lymphocytes depletion by CD20 monoclonal antibody prevents 136 
diabetes in NOD mice despite isotype-specific differences in Fc $\gamma \mathrm{R}$ effector functions. J. Immunol. 180:2863-2875.

52. Wolf, S. D., B. N. Dittel, F. Hardardottir, and C. A. Janeway, Jr. 1996. Experimental autoimmune encephalomyelitis induction in genetically B celldeficient mice. J. Exp. Med. 184:2271-2278.

53. Cross, A. H., J. L. Trotter, and J. Lyons. 2001. B cells and antibodies in CNS demyelinating disease. J. Neuroimmunol. 112:1-14.

54. Du, C., and S. Sriram. 2002. Increased severity of experimental allergic encephalomyelitis in $1 \mathrm{yn}^{-/-}$mice in the absence of elevated proinflammatory cytokine response in the central nervous system. J. Immunol. 168:3105-3112.

55. Fillatreau, S., C. H. Sweenie, M. J. McGeachy, D. Gray, and S. M. Anderton. 2002. B cells regulate autoimmunity by provision of IL-10. Nat. Immunol. 3:944-950.

56. Bettelli, E., D. Baeten, A. Jager, R. A. Sobel, and V. K. Kuchroo. 2006. Myelin oligodendrocyte glycoprotein-specific $\mathrm{T}$ and B cells cooperate to induce a Deviclike disease in mice. J. Clin. Invest. 116:2393-2402.

57. Krishnamoorthy, G., H. Lassmann, H. Wekerle, and A. Holz. 2006. Spontaneous opticospinal encephalomyelitis in a double-transgenic mouse model of autoimmune T cell/ B cell cooperation. J. Clin. Invest. 116:2385-2392.

58. Matsushita, T., K. Yanaba, J.-D. Bouaziz, M. Fujimoto, and T. F. Tedder. 2008. Regulatory B cells inhibit EAE initiation in mice while other B cells promote disease progression. J. Clin. Invest. 118:3420-3430.

59. Shlomchik, M. J., M. P. Madaio, D. Ni, M. Trounstine, and D. Huszar. 1994. The role of B cells in lpr/lpr-induced autoimmunity. J. Exp. Med. 180:1295-1306.

60. Chan, O., and M. J. Shlomchik. 1998. A new role for B cells in systemic autoimmunity: B cells promote spontaneous T cell activation in MRL-lpr/lpr mice. J. Immunol. 160:51-59.

61. Katz, S. I., D. Parker, and J. L. Turk. 1974. B-cell suppression of delayed hypersensitivity reactions. Nature 251:550-551.

62. Neta, R., and S. B. Salvin. 1974. Specific suppression of delayed hypersensitivity: the possible presence of a suppressor B cell in the regulation of delayed hypersensitivity. J. Immunol. 113:1716-1725.

63. Mizoguchi, A., and A. K. Bhan. 2006. A case for regulatory B cells. J. Immunol. 176:705-710.

64. Serra, P., and P. Santamaria. 2006. To 'B' regulated: B cells as members of the regulatory workforce. Trends Immunol. 27:7-10.

65. Mauri, C., and M. R. Ehrenstein. 2008. The 'short' history of regulatory B cells. Trends Immunol. 29:34-40. 
66. Lund, F. E. 2008. Cytokine-producing B lymphocytes-key regulators of immunity. Curr. Opin. Immunol. 20:332-338.

67. Bouaziz, J.-D., K. Yanaba, and T. F. Tedder. 2008. Regulatory B cells as inhibitors of immune responses and inflammation. Immunol. Rev. 224:201-214.

68. Yanaba, K., J.-D. Bouaziz, K. M. Haas, J. C. Poe, M. Fujimoto, and T. F. Tedder. 2008. A regulatory B cell subset with a unique $C D 1 \mathrm{~d}^{\text {hi }} C D 5^{+}$phenotype controls $\mathrm{T}$ cell-dependent inflammatory responses. Immunity 28:639-650.

69. Yanaba, K., J.-D. Bouaziz, T. Matsushita, T. Tasubata, and T. F. Tedder. 2009. The development and function of regulatory B cells expressing IL-10 (B10 cells) requires antigen receptor diversity and TLR signals. J. Immunol. 182:7459-7472.

70. Bursch, L. S., L. Wang, B. Igyarto, A. Kissenpfennig, B. Malissen, D. H. Kaplan, and K. A. Hogquist. 2007. Identification of a novel population of Langerin ${ }^{+}$ dendritic cells. J. Exp. Med. 204:3147-3156.

71. Uchida, J., Y. Hamaguchi, J. A. Oliver, J. V. Ravetch, J. C. Poe, K. M. Haas, and T. F. Tedder. 2004. The innate mononuclear phagocyte network depletes B lymphocytes through $F_{c}$ receptor-dependent mechanisms during anti-CD20 antibody immunotherapy. J. Exp. Med. 199:1659-1669.

72. Hamaguchi, Y., Y. Xiu, K. Komura, F. Nimmerjahn, and T. F. Tedder. 2006. Antibody isotype-specific engagement of Fc $\gamma$ receptors regulates B lymphocyte depletion during CD20 immunotherapy. J. Exp. Med. 203:743-753.

73. Matsushita, T., M. Horikawa, Y. Iwata, and T. F. Tedder. 2010. Regulatory B cells (B10 cells) and regulatory T cells have independent roles in controlling EAE initiation and late-phase immunopathogenesis. J. Immunol. 185:(in press).

74. Lampropoulou, V., K. Hoehlig, T. Roch, P. Neves, E. Calderon Gomez, C. H. Sweenie, Y. Hao, A. A. Freitas, U. Steinhoff, S. M. Anderton, and S. Fillatreau. 2008. TLR-activated B cells suppress T cell-mediated autoimmunity. J. Immunol. 180:4763-4773.

75. Mizoguchi, A., E. Mizoguchi, R. N. Smith, F. I. Preffer, and A. K. Bhan. 1997. Suppressive role of B cells in chronic colitis of $\mathrm{T}$ cell receptor $\alpha$ mutant mice. J. Exp. Med. 186:1749-1756.

76. Mizoguchi, A., E. Mizoguchi, H. Takedatsu, R. S. Blumberg, and A. K. Bhan. 2002. Chronic intestinal inflammatory condition generates IL-10-producing regulatory B cell subset characterized by CD1d upregulation. Immunity 16:219230.

77. Wei, B., P. Velazquez, O. Turovskaya, K. Spricher, R. Aranda, M. Kronenberg, L. Birnbaumer, and J. Braun. 2005. Mesenteric B cells centrally inhibit CD4 ${ }^{+} \mathrm{T}$ cell colitis through interaction with regulatory T cell subsets. Proc. Natl. Acad. Sci. U S A 102:2010-2015. 
78. Hussain, S., and T. L. Delovitch. 2007. Intravenous transfusion of BCR-activated B cells protects NOD mice from type 1 diabetes in an IL-10-dependent manner. J. Immunol. 179:7225-7232.

79. Tian, J., D. Zekzer, L. Hanssen, Y. Lu, A. Olcott, and D. L. Kaufman. 2001. Lipopolysaccharide-activated B cells down-regulate Th1 immunity and prevent autoimmune diabetes in nonobese diabetic mice. J. Immunol. 167:1081-1089.

80. Trentham, D. E., A. S. Townes, and A. H. Kang. 1977. Autoimmunity to type II collagen an experimental model of arthritis. J. Exp. Med. 146:857-868.

81. Mauri, C., D. Gray, N. Mushtaq, and M. Londei. 2003. Prevention of arthritis by interleukin 10-producing B cells. J. Exp. Med. 197:489-501.

82. Evans, J. G., K. A. Chavez-Rueda, A. Eddaoudi, A. Meyer-Bahlburg, D. J. Rawlings, M. R. Ehrenstein, and C. Mauri. 2007. Novel suppressive function of transitional 2 B cells in experimental arthritis. J. Immunol. 178:7868-7878.

83. Inoue, S., W. W. Leitner, B. Golding, and D. Scott. 2006. Inhibitory effects of B cells on antitumor immunity. Cancer Res. 66:7741-7747.

84. Shah, S., A. A. Divekar, S. P. Hilchey, H. M. Cho, C. L. Newman, S. U. Shin, H. Nechustan, P. M. Challita-Eid, B. M. Segal, K. H. Yi, and J. D. Rosenblatt. 2005. Increased rejection of primary tumors in mice lacking B cells: inhibition of antitumor CTL and TH1 cytokine responses by B cells. Int. J. Cancer 117:574-586.

85. Qin, Z., G. Richter, T. Schuler, S. Ibe, X. Cao, and T. Blankenstein. 1998. B cells inhibit induction of T cell-dependent tumor immunity. Nat. Med. 4:627-630.

86. Radbruch, A., G. Muehlinghaus, E. O. Luger, A. Inamine, K. G. Smith, T. Dorner, and F. Hiepe. 2006. Competence and competition: the challenge of becoming a long-lived plasma cell. Nature Rev. Immunol. 6:741-750.

87. Amanna, I. J., and M. K. Slifka. Mechanisms that determine plasma cell lifespan and the duration of humoral immunity. Immunological reviews 236:125-138.

88. Tew, J. G., and T. E. Mandel. 1979. Prolonged antigen half-life in the lymphoid follicles of specifically immunized mice. Immunology 37:69-76.

89. Tew, J. G., T. E. Mandel, and A. W. Burgess. 1979. Retention of intact HSA for prolonged periods in the popliteal lymph nodes of specifically immunized mice. Cellular immunology 45:207-212.

90. Zinkernagel, R. M., M. F. Bachmann, T. M. Kundig, S. Oehen, H. Pirchet, and H. Hengartner. 1996. On immunological memory. Annu Rev Immunol 14:333-367.

91. Bernasconi, N. L., E. Traggiai, and A. Lanzavecchia. 2002. Maintenance of serological memory by polyclonal activation of human memory B cells. Science 298:2199-2202. 
92. Traggiai, E., R. Puzone, and A. Lanzavecchia. 2003. Antigen dependent and independent mechanisms that sustain serum antibody levels. Vaccine 21 Suppl 2:S35-37.

93. Slifka, M. K., R. Antia, J. K. Whitmire, and R. Ahmed. 1998. Humoral immunity due to long-lived plasma cells. Immunity 8:363-372.

94. Slifka, M. K., and R. Ahmed. 1998. Long-lived plasma cells: a mechanism for maintaining persistent antibody production. Curr. Opinion Immunol. 10:252-258.

95. Manz, R. A., S. Arce, G. Cassese, A. E. Hauser, F. Hiepe, and A. Radbruch. 2002. Humoral immunity and long-lived plasma cells. Curr. Opin. Immunol. 14:517-521.

96. Manz, R. A., A. Thiel, and A. Radbruch. 1997. Lifetime of plasma cells in the bone marrow. Nature 388:133-134.

97. Manz, R. A., M. Lohning, G. Cassese, A. Thiel, and A. Radbruch. 1998. Survival of long-lived plasma cells is independent of antigen. Intl. Immunol. 10:1703-1711.

98. Benner, R., W. Hijmans, and J. J. Haaijman. 1981. The bone marrow: the major source of serum immunoglobulins, but still a neglected site of antibody formation. Clin. Exp. Immunol. 46:1-8.

99. Benson, M. J., S. R. Dillon, E. Castigli, R. S. Geha, S. Xu, K. P. Lam, and R. J. Noelle. 2008. Cutting edge: the dependence of plasma cells and independence of memory B cells on BAFF and APRIL. J Immunol 180:3655-3659.

100. Barnden, M. J., J. Allison, W. R. Heath, and F. R. Carbone. 1998. Defective TCR expression in transgenic mice constructed using cDNA-based $\alpha$ - and $\beta$-chain genes under the control of heterologous regulatory elements. Immunol. Cell. Biol. 76:34-40.

101. Hogquist, K. A., S. C. Jameson, W. R. Heath, J. L. Howard, M. J. Bevan, and F. R. Carbone. 1994. T cell receptor antagonist peptides induce positive selection. Cell 76:17-27.

102. Zhou, L.-J., H. M. Smith, T. J. Waldschmidt, R. Schwarting, J. Daley, and T. F. Tedder. 1994. Tissue-specific expression of the human CD19 gene in transgenic mice inhibits antigen-independent B lymphocyte development. Mol. Cell. Biol. 14:3884-3894.

103. Engel, P., L.-J. Zhou, D. C. Ord, S. Sato, B. Koller, and T. F. Tedder. 1995. Abnormal B lymphocyte development, activation and differentiation in mice that lack or overexpress the CD19 signal transduction molecule. Immunity 3:39-50.

104. Uchida, J., Y. Lee, M. Hasegawa, Y. Liang, A. Bradney, J. A. Oliver, K. Bowen, D. A. Steeber, K. M. Haas, J. C. Poe, and T. F. Tedder. 2004. Mouse CD20 expression and function. Int. Immunol. 16:119-129.

105. Steeber, D. A., P. Engel, A. S. Miller, M. P. Sheetz, and T. F. Tedder. 1997. Ligation of L-selectin through conserved regions within the lectin domain activates 
signal transduction pathways and integrin function in human, mouse and rat leukocytes. J. Immunol. 159:952-963.

106. Dal Porto, J., A. Haberman, M. Shlomchik, and G. Kelsoe. 1998. Antigen drives very low affinity $B$ cells to become plasmacytes and enter germinal centers. J. Immunol. 161:5373-5381.

107. Sato, S., N. Ono, D. A. Steeber, D. S. Pisetsky, and T. F. Tedder. 1996. CD19 regulates B lymphocyte signaling thresholds critical for the development of B-1 lineage cells and autoimmunity. J. Immunol. 157:4371-4378.

108. Soro, P. G., A. P. Morales, M. J. Martinez, A. S. Morales, S. G. Copin, M. A. Marcos, and M. L. Gaspar. 1999. Differential involvement of the transcription factor Blimp-1 in T cell-independent and -dependent B cell differentiation to plasma cells. J. Immunol. 163:611-617.

109. Brown, D. M., T. L. Fisher, C. Wei, J. G. Frelinger, and E. M. Lord. 2001. Tumours can act as adjuvants for humoral immunity. Immunology 102:486-497.

110. Ehst, B. D., E. Ingulli, and M. K. Jenkins. 2003. Development of a novel transgenic mouse for the study of interactions between CD4 and CD8 T cells during graft rejection. Am. J. Transplant. 3:1355-1362.

111. Mannon, R. B., B. L. Kotzin, C. Nataraj, K. Ferri, E. Roper, R. J. Kurlander, and T. M. Coffman. 1998. Downregulation of T cell receptor expression by CD8(+) lymphocytes in kidney allografts. J. Clin. Invest. 101:2517-2527.

112. Billingham, R. E. 1961. Free skin grafting in mammals. In Transplantation of Tissues and Cells. R. E. Billingham, and W. K. Silvers, eds. The Wistar Institute Press, Philadelphia. 1-24.

113. Coffman, T., S. Geier, S. Ibrahim, R. Griffiths, R. Spurney, O. Smithies, B. Koller, and F. Sanfilippo. 1993. Improved renal function in mouse kidney allografts lacking MHC class I antigens. J. Immunol. 151:425-435.

114. Spurney, R. F., S. Ibrahim, D. Butterly, P. E. Klotman, F. Sanfilippo, and T. M. Coffman. 1994. Leukotrienes in renal transplant rejection in rats. Distinct roles for leukotriene B4 and peptidoleukotrienes in the pathogenesis of allograft injury. J. Immunol. 152:867-876.

115. Spurney, R. F., P. Ruiz, D. S. Pisetsky, and T. M. Coffman. 1991. Enhanced renal leukotriene production in murine lupus: role of lipoxygenase metabolites. Kidney Int. 39:95-102.

116. DiLillo, D. J., T. Matsushita, and T. F. Tedder. 2010. B10 cells and regulatory B cells balance immune responses during inflammation, autoimmunity, and cancer. Ann. N. Y. Acad. Sci. 1183:38-57.

117. Coughlin, C. M., B. A. Vance, S. A. Grupp, and R. H. Vonderheide. 2004. RNAtransfected CD40-activated B cells induce functional T-cell responses against 
viral and tumor antigen targets: implications for pediatric immunotherapy. Blood 103:2046-2054.

118. Gordon, J., H. T. Holden, S. Segal, and M. Feldman. 1982. Anti-tumor immunity in B-lymphocyte-deprived mice. III. Immunity to primary Moloney sarcoma virusinduced tumors. Int. J. Cancer 29:351-357.

119. Schultz, K. R., J. P. Klarnet, R. S. Gieni, K. T. HayGlass, and P. D. Greenberg. 1990. The role of B cells for in vivo T cell responses to a Friend virus-induced leukemia. Science 249:921-923.

120. Manson, L. A. 1994. Anti-tumor immune responses of the tumor-bearing host: the case for antibody-mediated immunologic enhancement. Clin. Immunol. Immunopathol. 72:1-8.

121. Houghton, A. N., H. Uchi, and J. D. Wolchok. 2005. The role of the immune system in early epithelial carconogenesis: B-ware the double-edged sword. Cancer Cell 7:403-405.

122. de Visser, K. E., L. V. Korets, and L. M. Coussens. 2005. De novo carcinogenesis promoted by chronic inflammation is B lymphocyte dependent. Cancer Cell 7:411423.

123. Schreiber, H., T. H. Wu, J. Nachman, and D. A. Rowley. 2000. Immunological enhancement of primary tumor development and its prevention. Semin. Cancer Biol. 10:351-357.

124. Brodt, P., and J. Gordon. 1978. Anti-tumor immunity in B lymphocyte-deprived mice. I. Immunity to a chemically induced tumor. J. Immunol. 121:359-362.

125. Barbera-Guillem, E., M. B. Nelson, B. Barr, J. K. Nyhus, K. F. May, Jr., L. Feng, and J. W. Sampsel. 2000. B lymphocyte pathology in human colorectal cancer. Experimental and clinical therapeutic effects of partial B cell depletion. Cancer Immunol. Immunother. 48:541-549.

126. Monach, P. A., H. Schreiber, and D. A. Rowley. 1993. CD $4^{+}$and B lymphocytes in transplantation immunity. II. Augmented rejection of tumor allografts by mice lacking B cells. Transplantation 55:1356-1361.

127. Asano, M. S., and R. Ahmed. 1996. CD8 T cell memory in B cell-deficient mice. J. Exp. Med. 183:2165-2174.

128. Yanaba, K., J. D. Bouaziz, T. Matsushita, C. M. Magro, E. W. St Clair, and T. F. Tedder. 2008. B-lymphocyte contributions to human autoimmune disease. Immunol. Rev. 223:284-299.

129. Overwijk, W. W., M. R. Theoret, S. E. Finkelstein, D. R. Surman, L. A. de Jong, F. A. Vyth-Dreese, T. A. Dellemijn, P. A. Antony, P. J. Spiess, D. C. Palmer, D. M. Heimann, C. A. Klebanoff, Z. Yu, L. N. Hwang, L. Feigenbaum, A. M. Kruisbeek, S. A. Rosenberg, and N. P. Restifo. 2003. Tumor regression and autoimmunity 
after reversal of a functionally tolerant state of self-reactive CD8 ${ }^{+} \mathrm{T}$ cells. J. Exp. Med. 198:569-580.

130. Fidler, I. J. 1973. Selection of successive tumour lines for metastasis. Nat. New Biol. 242:148-149.

131. Seliger, B., U. Wollscheid, F. Momburg, T. Blankenstein, and C. Huber. 2001. Characterization of the major histocompatibility complex class I deficiencies in B16 melanoma cells. Cancer Res. 61:1095-1099.

132. Nakamura, K., N. Yoshikawa, Y. Yamaguchi, S. Kagota, K. Shinozuka, and M. Kunitomo. 2002. Characterization of mouse melanoma cell lines by their mortal malignancy using an experimental metastatic model. Life Sci. 70:791-798.

133. Hasegawa, M., Y. Hamaguchi, K. Yanaba, J.-D. Bouaziz, J. Uchida, M. Fujimoto, T. Matsushita, Y. Matsushita, M. Horikawa, K. Komura, K. Takehara, S. Sato, and T. F. Tedder. 2006. B-lymphocyte depletion reduces skin fibrosis and autoimmunity in the tight-skin mouse model for systemic sclerosis. Am. J. Pathol. 169:954-966.

134. Dobrzanski, M. J., J. B. Reome, and R. W. Dutton. 2000. Type 1 and type 2 CD $8^{+}$ effector $\mathrm{T}$ cell subpopulations promote long-term tumor immunity and protection to progressively growing tumor. J. Immunol. 164:916-925.

135. Sfondrini, L., D. Morelli, A. Bodini, M. I. Colnaghi, S. Menard, and A. Balsari. 1999. High level antibody response to retrovirus-associated but not to melanocyte lineage-specific antigens in mice protected against B16 melanoma. Int. J. Cancer 83:107-112.

136. Preynat-Seauve, O., E. Contassot, P. Schuler, V. Piguet, L. E. French, and B. Huard. 2007. Extralymphatic tumors prepare draining lymph nodes to invasion via a T-cell cross-tolerance process. Cancer Res. 67:5009-5016.

137. Stoitzner, P., L. K. Green, J. Y. Jung, K. M. Price, H. Atarea, B. Kivell, and F. Ronchese. 2008. Inefficient presentation of tumor-derived antigen by tumorinfiltrating dendritic cells. Cancer Immunol. Immunother. 57:1665-1673.

138. Rivera, A., C. C. Chen, N. Ron, J. P. Dougherty, and Y. Ron. 2001. Role of B cells as antigen-presenting cells in vivo revisited: antigen-specific B cells are essential for $\mathrm{T}$ cell expansion in lymph nodes and for systemic $\mathrm{T}$ cell responses to low antigen concentrations. Int. Immunol. 13:1583-1593.

139. Hamel, K., P. Doodes, Y. Cao, Y. Wang, J. Martinson, R. Dunn, M. R. Kehry, B. Farkas, and A. Finnegan. 2008. Suppression of proteoglycan-induced arthritis by anti-CD20 B cell depletion therapy is mediated by reduction in autoantibodies and $\mathrm{CD}^{+}{ }^{+} \mathrm{T}$ cell reactivity. J. Immunol. 180:4994-5003.

140. Homann, D., A. Tishon, D. P. Berger, W. O. Weigle, M. G. von Herrath, and M. B. Oldstone. 1998. Evidence for an underlying CD4 helper and CD8 T-cell defect in B-cell-deficient mice: failure to clear persistent virus infection after adoptive 
immunotherapy with virus-specific memory cells from $\mu \mathrm{MT} / \mu \mathrm{MT}$ mice. J. Virol. 72:9208-9216.

141. Ritchie, D. S., J. Yang, I. F. Hermans, and F. Ronchese. 2004. B-Lymphocytes activated by CD40 ligand induce an antigen-specific anti-tumour immune response by direct and indirect activation of $\mathrm{CD} 8^{+} \mathrm{T}$-cells. Scand. J. Immunol. 60:543-551.

142. Ahuja, A., J. Shupe, R. Dunn, M. Kashgarian, M. R. Kehry, and M. J. Shlomchik. 2007. Depletion of B cells in murine lupus: efficacy and resistance. J. Immunol. 179:3351-3361.

143. Nozaki, T., J. M. Rosenblum, D. Ishii, K. Tanabe, and R. L. Fairchild. 2008. CD4 T cell-mediated rejection of cardiac allografts in B cell-deficient mice. J. Immunol. 181:5257-5263.

144. Perricone, M. A., K. A. Smith, K. A. Claussen, M. S. Plog, D. M. Hempel, B. L. Roberts, J. A. St. George, and J. M. Kaplan. 2004. Enhanced efficacy of melanoma vaccines in the absence of B lymphocytes. J. Immunother. 27:273-281.

145. Kim, S., Z. G. Fridlender, R. Dunn, M. R. Kehry, V. Kapoor, A. Blouin, L. R. Kaiser, and S. M. Albelda. 2008. B-cell depletion using an anti-CD20 antibody augments antitumor immune responses and immunotherapy in nonhematopoetic murine tumor models. J Immunother 31:446-457.

146. Neelapu, S. S., L. W. Kwak, C. B. Kobrin, C. W. Reynolds, J. E. Janik, K. Dunleavy, T. White, L. Harvey, R. Pennington, M. Stetler-Stevenson, E. S. Jaffe, S. M. Steinberg, R. Gress, F. Hakim, and W. H. Wilson. 2005. Vaccine-induced tumor-specific immunity despite severe B-cell depletion in mantle cell lymphoma. Nat. Med. 11:986-991.

147. Schultze, J. L., S. Grabbe, and M. S. von Bergwelt-Baildon. 2004. DCs and CD40activated B cells: current and future avenues to cellular cancer immunotherapy. Trends Immunol. 25:659-664.

148. O'Neill, S. K., Y. Cao, K. M. Hamel, P. D. Doodes, G. Hutas, and A. Finnegan. 2007. Expression of CD80/ 86 on B cells is essential for autoreactive T cell activation and the development of arthritis. J. Immunol. 179:5109-5116.

149. Watanabe, R., N. Ishiura, H. Nakashima, Y. Kuwano, H. Okochi, K. Tamaki, S. Sato, T. F. Tedder, and M. Fujimoto. 2010. Regulatory B cells (B10 cells) have a suppressive role in murine lupus: CD19 and B10 cell deficiency exacerbates systemic autoimmunity. J. Immunol. 184:4801-4809.

150. Brandle, D., J. Joergensen, G. Zenke, K. Burki, and R. P. Hof. 1998. Contribution of donor-specific antibodies to acute allograft rejection: evidence from B celldeficient mice. Transplantation 65:1489-1493.

151. Alexander, D. Z., T. C. Pearson, R. Hendrix, S. C. Ritchie, and C. P. Larsen. 1996. Analysis of effector mechanisms in murine cardiac allograft rejection. Transpl. Immunol. 4:46-48. 
152. Gareau, A., G. M. Hirsch, T. D. Lee, and B. Nashan. 2009. Contribution of B cells and antibody to cardiac allograft vasculopathy. Transplantation 88:470-477.

153. Noorchashm, H., A. J. Reed, S. Y. Rostami, R. Mozaffari, G. Zekavat, B. Koeberlein, A. J. Caton, and A. Naji. 2006. B cell-mediated antigen presentation is required for the pathogenesis of acute cardiac allograft rejection. J. Immunol. 177:7715-7722.

154. Wasowska, B. A., Z. Qian, D. L. Cangello, E. Behrens, K. Van Tran, J. Layton, F. Sanfilippo, and W. M. Baldwin, 3rd. 2001. Passive transfer of alloantibodies restores acute cardiac rejection in IgKO mice. Transplantation 71:727-736.

155. Nozaki, T., H. Amano, A. Bickerstaff, C. G. Orosz, A. C. Novick, K. Tanabe, and R. L. Fairchild. 2007. Antibody-mediated rejection of cardiac allografts in CCR5deficient recipients. J. Immunol. 179:5238-5245.

156. Yazawa, N., Y. Hamaguchi, J. C. Poe, and T. F. Tedder. 2005. Immunotherapy using unconjugated CD19 monoclonal antibodies in animal models for B lymphocyte malignancies and autoimmune disease. Proc. Natl. Acad. Sci. USA 102:15178-15183.

157. Tedder, T. F. 2009. CD19: a promising B cell target for rheumatoid arthritis. Nat. Rev. Rheumatol. 5:572-577.

158. Wijdenes, J., W. C. Vooijs, C. Clement, J. Post, F. Morard, N. Vita, P. Laurent, R. X. Sun, B. Klein, and J. M. Dore. 1996. A plasmocyte selective monoclonal antibody (B-B4) recognizes syndecan-1. Br. J. Haematol. 94:318-323.

159. Sanderson, R. D., P. Lalor, and M. Bernfield. 1989. B lymphocytes express and lose syndecan at specific stages of differentiation. Cell Reg. 1:27-35.

160. Hoyer, B. F., K. Moser, A. E. Hauser, A. Peddinghaus, C. Voigt, D. Eilat, A. Radbruch, F. Hiepe, and R. A. Manz. 2004. Short-lived plasmablasts and longlived plasma cells contribute to chronic humoral autoimmunity in NZB / W mice. J. Exp. Med. 199:1577-1584.

161. Tedder, T. F., and P. Engel. 1994. CD20: a regulator of cell-cycle progression of B lymphocytes. Immunol. Today 15:450-454.

162. Nadler, L. M., K. C. Anderson, G. Marti, M. Bates, E. Park, J. F. Daley, and S. F. Schlossman. 1983. B4, a human B lymphocyte-associated antigen expressed on normal, mitogen activated, and malignant B lymphocytes. J. Immunol. 131:244250.

163. Robinson, L. A., C. Nataraj, D. W. Thomas, D. N. Howell, R. Griffiths, V. Bautch, D. D. Patel, L. Feng, and T. M. Coffman. 2000. A role for fractalkine and its receptor (CX3CR1) in cardiac allograft rejection. J. Immunol. 165:6067-6072. 
164. Mannon, R. B., J. B. Kopp, P. Ruiz, R. Griffiths, M. Bustos, J. L. Platt, P. E. Klotman, and T. M. Coffman. 1999. Chronic rejection of mouse kidney allografts. Kidney Int. 55:1935-1944.

165. Michaels, P. J., M. C. Fishbein, and R. B. Colvin. 2003. Humoral rejection of human organ transplants. Springer Semin. Immunopathol. 25:119-140.

166. Wu, G. D., Y. He, N. N. Chai, M. Toyoda, R. Dunn, M. R. Kehry, A. S. Klein, and S. C. Jordan. 2008. Anti-CD20 antibody suppresses anti-HLA antibody formation in a HLA-A2 transgenic mouse model of sensitization. Transpl. Immunol. 19:178-186.

167. Patselas, T., W. Marchman, D. Araneda, J. Thomas, and F. Thomas. 1992. Normal rejection of xenografts by strongly deficient B-cell animals (X-linked immunodeficient mice). Transplant Proc. 24:494.

168. Cerny, A., H. Ramseier, H. Bazin, and R. M. Zinkernagel. 1988. Unimpaired firstset and second-set skin graft rejection in agammaglobulinemic mice.

Transplantation 45:1111-1113.

169. Winn, H. J., C. A. Baldamus, S. V. Jooste, and P. S. Russell. 1973. Acute destruction by humoral antibody of rat skin grafted to mice. J. Exp. Med. 137:893910.

170. Jooste, S. V., and H. J. Winn. 1975. Acute destruction of rat skin grafts by alloantisera. J. Immunol. 114:933-938.

171. Russell, P. S., C. M. Chase, H. J. Winn, and R. B. Colvin. 1994. Coronary atherosclerosis in transplanted mouse hearts. II. Importance of humoral immunity. J. Immunol. 152:5135-5141.

172. Rifle, G., C. Mousson, L. Martin, F. Guignier, and K. Hajji. 2005. Donor-specific antibodies in allograft rejection: clinical and experimental data. Transplantation 79:S14-18.

173. Vieira, C. A., A. Agarwal, B. K. Book, R. A. Sidner, C. M. Bearden, H. M. Gebel, A. L. Roggero, N. S. Fineberg, T. Taber, M. A. Kraus, and M. D. Pescovitz. 2004. Rituximab for reduction of anti-HLA antibodies in patients awaiting renal transplantation: 1 . Safety, pharmacodynamics, and pharmacokinetics. Transplantation 77:542-548.

174. Claas, F. H., and Doxiadis, II. 2009. Management of the highly sensitized patient. Curr. Opin. Immunol. 21:569-572.

175. Singh, N., J. Pirsch, and M. Samaniego. 2009. Antibody-mediated rejection: treatment alternatives and outcomes. Transplant Rev. 23:34-46.

176. Vo, A. A., M. Lukovsky, M. Toyoda, J. Wang, N. L. Reinsmoen, C. H. Lai, A. Peng, R. Villicana, and S. C. Jordan. 2008. Rituximab and intravenous immune globulin for desensitization during renal transplantation. N. Engl. J. Med. 359:242251. 
177. Stegall, M. D., J. Gloor, J. L. Winters, S. B. Moore, and S. Degoey. 2006. A comparison of plasmapheresis versus high-dose IVIG desensitization in renal allograft recipients with high levels of donor specific alloantibody. Am. J. Transplant. 6:346-351.

178. Garrett, H. E., Jr., D. Duvall-Seaman, B. Helsley, and K. Groshart. 2005. Treatment of vascular rejection with rituximab in cardiac transplantation. J. Heart Lung Transplant. 24:1337-1342.

179. Becker, Y. T., B. N. Becker, J. D. Pirsch, and H. W. Sollinger. 2004. Rituximab as treatment for refractory kidney transplant rejection. Am. J. Transplant. 4:9961001.

180. Clatworthy, M. R., C. J. Watson, G. Plotnek, V. Bardsley, A. N. Chaudhry, J. A. Bradley, and K. G. Smith. 2009. B-cell-depleting induction therapy and acute cellular rejection. N. Engl. J. Med. 360:2683-2685.

181. Haas, K. M., J. C. Poe, D. A. Steeber, and T. F. Tedder. 2005. B-1a and B-1b cells exhibit distinct developmental requirements and have unique functional roles in innate and adaptive immunity to S. pneumoniae. Immunity 23:7-18.

182. Kantor, A. B., and L. A. Herzenberg. 1993. Origin of murine B cell lineages. Ann. Rev. Immunol. 11:501-538.

183. Martin, F., and J. F. Kearney. 2002. Marginal-zone B cells. Nat. Rev. Immunol. 2:323-335.

184. Kelsoe, G. 1996. Life and death in germinal centers (redux). Immunity 4:107-111.

185. Jacob, R., R. Kassir, and G. Kelsoe. 1991. In situ studies of the primary immune response to (4-hydroxy-3-nitrophenyl)acetyl. I. The architecture and dynamics of responding cell populations. J. Exp. Med. 173:1165-1175.

186. Hayakawa, K., R. Ishii, K. Yamasaki, T. Kishimoto, and R. R. Hardy. 1987. Isolation of high-affinity memory B cells: phycoerythrin as a probe for antigenbinding cells. Proc. Natl. Acad. Sci. USA 84:1379-1383.

187. Black, J. J., T. Tokuhisa, L. A. Herzenberg, and L. A. Herzenberg. 1980. Memory B cells at successive stages of differentiation: Expression of surface $\operatorname{IgD}$ and capacity for self-renewal. Eur. J. Immunol. 10:846-851.

188. Herzenberg, L. A., S. J. Black, T. Tokuhisa, and L. A. Herzenberg. 1980. Memory $B$ cells at successive stages of differentiation. Affinity maturation and the role of IgD receptors. J. Exp. Med. 151:1071-1087.

189. O'Connor, B. P., M. Cascalho, and R. J. Noelle. 2002. Short-lived and long-lived bone marrow plasma cells are derived from a novel precursor population. J. Exp. Med. 195:737-745. 
190. Smith, M. R. 2003. Rituximab (monoclonal anti-CD20 antibody): mechanisms of action and resistance. Oncogene 22:7359-7368.

191. Martin, F., and A. C. Chan. 2004. Pathogenic roles of B cells in human autoimmunity: insights from the clinic. Immunity 20:517-527.

192. Hayakawa, I., T. F. Tedder, and Y. Zhuang. 2007. B-lymphocyte depletion ameliorates Sjogren's syndrome in Id3 knockout mice. Immunology 122:73-79.

193. Hasbold, J., L. M. Corcoran, D. M. Tarlinton, S. G. Tangye, and P. D. Hodgkin. 2004. Evidence from the generation of immunoglobulin G-secreting cells that stochastic mechanisms regulate lymphocyte differentiation. Nat. Immunol. 5:5563.

194. Underhill, G. H., H. A. Minges Wols, J. L. Fornek, P. L. Witte, and G. S. Kansas. 2002. IgG plasma cells display a unique spectrum of leukocyte adhesion and homing molecules. Blood 99:2905-2912.

195. Kallies, A., J. Hasbold, D. M. Tarlinton, W. Dietrich, L. M. Corcoran, P. D. Hodgkin, and S. L. Nutt. 2004. Plasma cell ontogeny defined by quantitative changes in blimp-1 expression. J. Exp. Med. 200:967-977.

196. Lu, T. T., and J. G. Cyster. 2002. Integrin-mediated long-term B cell retention in the splenic marginal zone. Science 297:409-412.

197. Hardy, R. R., and K. Hayakawa. 2001. B cell development pathways. Annu. Rev. Immunol. 19:595-621.

198. van der Kolk, L. E., J. W. Baars, M. H. Prins, and M. H. J. van Oers. 2002. Rituximab treatment results in impaired secondary humoral immune responsiveness. Blood 100:2257-2259.

199. McLaughlin, P., L. A. Grillo, B. K. Link, R. Levy, M. S. Czuczman, M. E. Williams, M. R. Heyman, B. I. Bence, C. A. White, F. Cabanillas, V. Jain, A. D. Ho, J. Lister, K. Wey, D. Shen, and B. K. Dallaire. 1998. Rituximab chimeric anti-CD20 monoclonal antibody therapy for relapsed indolent lymphoma: half of patients respond to a four-dose treatment program. J. Clin. Oncol. 16:2825-2833.

200. Davis, T. A., A. J. Grillo-Lopez, C. A. White, P. McLaughlin, M. S. Czuczman, B. K. Link, D. G. Maloney, R. L. Weaver, J. Rosenberg, and R. Levy. 2000. Rituximab anti-CD20 monoclonal antibody therapy in non-Hodgkin's lymphoma: safety and efficacy of re-treatment. J. Clin. Oncol. 18:3135-3143.

201. Cambridge, G., M. J. Leandro, J. C. W. Edwards, M. R. Ehrenstein, M. Salden, M. Bodman-Smith, and A. D. B. Webster. 2003. Serologic changes following B lymphocyte depletion therapy for rheumatoid arthritis. Arthritis Rheum. 48:21462154.

202. Gonzalez-Stawinski, G. V., P. B. Yu, L. S. D., W. Parker, and R. D. Davis Jr. 2001. Hapten-induced primary and memory humoral responses are inhibited by the 
infusion of anti-CD20 monoclonal antibody (IDEC-C2B8, Rituximab). Clin. Immunol. 98:175-179.

203. Edwards, J. C. W., and G. Cambridge. 2001. Sustained improvement in rheumatoid arthritis following a protocol designed to deplete B lymphocytes. Rheumatology 40:205-211.

204. Stasi, R., A. Pagano, E. Stipa, and S. Amadori. 2001. Rituximab chimeric antiCD20 monoclonal antibody treatment for adults with chronic idiopathic thrombocytopenic purpura. Blood 98:952-957.

205. Specks, U., F. C. Fervenza, T. J. McDonald, and M. C. E. Hogan. 2001. Response of Wegener's granulomatosus to anti-CD20 chimeric monoclonal antibody therapy. Arthritis Rheum. 44:2836-2840.

206. Levine, T. D., and A. Pestronk. 1999. IgM antibody-related polyneuropathies: Bcell depletion chemotherapy using Rituximab. Neurology 12:1701-1704.

207. De Vita, S. D., F. Zaja, S. Sacco, A. D. Candia, R. Fanin, and G. Ferraccioli. 2002. Efficacy of selective B cell blockade in the treatment of rheumatoid arthritis: evidence for a pathogenic role of B cells. Arthritis Rheum. 46:2029-2033.

208. Patel, D. D. 2002. B cell-ablative therapy for the treatment of autoimmune diseases. Arthritis Rheum. 46:1984-1985.

209. Vieira, P., and K. Rajewsky. 1988. The half-lives of serum immunoglobulins in adult mice. Eur. J. Immunol. 18:313-316.

210. Chan, O. T., L. G. Hannum, A. M. Haberman, M. P. Madaio, and M. J. Shlomchik. 1999. A novel mouse with B cells but lacking serum antibody reveals an antibodyindependent role for B cells in murine lupus. J. Exp. Med. 189:1639-1648.

211. Pillai, S., A. Cariappa, and S. T. Moran. 2005. Marginal zone B cells. Annu Rev Immunol 23:161-196.

212. Oliver, A. M., F. Martin, G. L. Gartland, R. H. Carter, and J. F. Kearney. 1997. Marginal zone B cells exhibit unique activation, proliferative and immunoglobulin secretory responses. Eur. J. Immunol. 27:2366-2374.

213. Oliver, A. M., F. Martin, and J. F. Kearney. 1999. IgM ${ }^{\text {high }} C D 21^{\text {high }}$ lymphocytes enriched in the splenic marginal zone generate effector cells more rapidly than the bulk of follicular B cells. J. Immunol. 162:7198-7207.

214. Attanavanich, K., and J. F. Kearney. 2004. Marginal zone, but not follicular B cells, are potent activators of naive CD4 T cells. J. Immunol. 172:803-811.

215. Eming, R., A. Nagel, S. Wolff-Franke, E. Podstawa, D. Debus, and M. Hertl. 2008. Rituximab exerts a dual effect in pemphigus vulgaris. J. Invest. Dermatol. 128:2850-2858. 
216. Vigna-Perez, M., B. Hernandez-Castro, O. Paredes-Saharopulos, D. PortalesPerez, L. Baranda, C. Abud-Mendoza, and R. Gonzalez-Amaro. 2006. Clinical and immunological effects of Rituximab in patients with lupus nephritis refractory to conventional therapy: a pilot study. Arthritis. Res. Ther. 8:R83-91.

217. Levesque, M. C., and E. W. St. Clair. 2008. B cell-directed therapies for autoimmune disease and correlates of disease response and relapse. J. Allergy Clin. Immunol. 121:13-21.

218. Liossis, S. N., and P. P. Sfikakis. 2008. Rituximab-induced B cell depletion in autoimmune diseases: potential effects on T cells. Clin. Immunol. 127:280-285. 


\section{Biography}

I was born on June, 7, 1982 in Vienna, VA, USA to John and MaryAnn DiLillo. I attended Our Lady of Good Counsel elementary and middle schools in Vienna, VA and graduated with an Advanced Studies Diploma from James Madison High School in (Vienna, VA) in June, 2000. I went on to study Biology at the University of Virginia, Charlottesville, and graduated with Highest Distinction in May of 2004. While at the University of Virginia, I performed undergraduate thesis research in the laboratory of Dr. Ronald P. Taylor, where I studied the role of the complement system in the mAbinduced killing of malignant B lymphocytes as well as the mechanisms regulating the alternative pathway of complement. Upon graduation from the University of Virginia, I was accepted into the Immunology Ph.D. program at Duke University, Durham, NC, where I spent the next six years studying B lymphocyte biology with Dr. Thomas F.

Tedder. I have published the following manuscripts in peer-reviewed journals:

DiLillo DI, Griffiths RC, Seshan SV, Magro C, Ruiz P, Coffman TM, Tedder TF. B lymphocytes differentially influence acute and chronic allograft rejection in mice. Manuscript in Revision, J. Immunol.

DiLillo DI Yanaba K, Tedder TF. B Cells Are Required for CD4 ${ }^{+}$and CD8 ${ }^{+}$T Cell Tumor Immunity: Therapeutic B Cell Depletion Enhances B16 Melanoma Growth in Mice. J. Immunol. 2010; 184(7):4006-16.

DiLillo DI, Matsushita T, and Tedder TF. B10 Cells and Regulatory B Cells Balance Immune Responses During Inflammation, Autoimmunity, and Cancer. Ann. N.Y. Acad. Sci. 2010; 1183:38-57.

DiLillo DI, Hamaguchi Y, Ueda Y, Yang K, Uchida J, Haas KM, Kelsoe G, and Tedder TF. Maintenance of Long-Lived Plasma Cells and Serological Memory Despite Mature and Memory B Cell Depletion During CD20 Immunotherapy In Mice. J. Immunol. 2008; 180(1): 361-371.

DiLillo DI, Pawluczkowycz AW, Peng W, Kennedy AD, Beum PV, Lindorfer MA, Taylor RP. Selective and efficient inhibition of the alternative pathway of complement by a mAb that recognizes C3b/iC3b. Mol. Immunol. 2006; 43(7): 10109.

Kennedy AD, Beum PV, Solga MD, DiLillo DI, Lindorfer MA, Hess CE, Densmore JJ, Williams ME, Taylor RP. Rituximab infusion promotes rapid complement depletion 
and acute CD20 loss in chronic lymphocytic leukemia. J. Immunol. 2004; 172(5): 3280-8.

I have recived the following awards and honors:

Jefferson Scholars Alumni Scholarship 2000-2004

Speak Award, 2004 University of Virginia Katz Research Symposium

Finalist, MedImmune, Inc. 2006 Inflammation and Autoimmunity Abstract Competition Abstract/Speaking Award, 2007 Biomedical Graduate Student Symposium, Duke University

Abstract/Poster Award, 2009 Biomedical Graduate Student Symposium, Duke University

Trainee Abstract Award, 2009 American Association of Immunologists Meeting, Seattle,WA

Speaker, Block Symposium, 2009 American Association of Immunologists Meeting, Seattle, WA

2009 Conference Travel Fellowship, Duke University Graduate School

Trainee Abstract Award, 2010 American Association of Immunologists Meeting, Baltimore, MD

Speaker, Block Symposium, 2010 American Association of Immunologists Meeting, Baltimore, MD

2010 Conference Travel Fellowship, Duke University Graduate School

Invited Participant and Speaker, RIKEN Institute Research Center for Allergy and Immunology 2010 Summer Program, Yokohama, Japan

Best Presentation Award, RIKEN Institute Research Center for Allergy and Immunology 2010 Summer Program, Yokohama, Japan

Abstract Presenter, 2010 International Conference on Immunology, Kobe, Japan

On October 8, 2010, I defended my dissertation with the faculty of the Duke University Department of Immunology. I am married to my wife Meghan, who also works at Duke University, and we currently reside in Durham, NC. 